\title{
Enantioselective Radical Carbocyanation of 1,3-Dienes via Photocatalytic Generation of Allylcopper Complexes
}

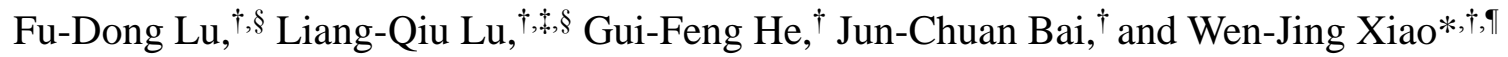 \\ 'CCNU-uOttawa Joint Research Centre, Key Laboratory of Pesticide and Chemical Biology, Ministry of Education, \\ College of Chemistry, Central China Normal University, 152 Luoyu Road, Wuhan 430079, China \\ ${ }^{\text {t}}$ State Key Laboratory for Oxo Synthesis and Selective Oxidation, Lanzhou Institute of Chemical Physics (LICP), Chinese \\ Academy of Sciences, Lanzhou 730000, People's Republic of China \\ "State Key Laboratory of Organometallic Chemistry, Shanghai Institute of Organic Chemistry, Chinese Academy of \\ Sciences, Shanghai 200032, China \\ ${ }^{\S}$ F.-D. Lu and L.-Q. Lu contributed equally to this work.
}

\section{Table of Contents}

1. General Information

2. Preparation and Characterization of Materials

3. Details for Condition Optimizations 


\section{General Information}

NMR spectra: ${ }^{1} \mathrm{H}$ NMR spectra were recorded on a 400/600 MHz spectrometer. Chemical shifts are reported in parts per million (ppm) and the spectra are calibrated to the resonance resulting from incomplete deuteration of the solvent ( $\left.\mathrm{CDCl}_{3}: 7.26 \mathrm{ppm}, \mathrm{CD}_{3} \mathrm{OD}: 3.31 \mathrm{ppm}, \mathrm{CD}_{2} \mathrm{Cl}_{2}: 5.17 \mathrm{ppm}\right) .{ }^{13} \mathrm{C}$ NMR spectra were recorded on the same spectrometer with complete proton decoupling. Chemical shifts are reported in ppm with the solvent resonance as the internal standard $\left({ }^{13} \mathrm{CDCl}_{3}: 77.0 \mathrm{ppm}\right.$, $\mathrm{t}$; ${ }^{13} \mathrm{CD}_{2} \mathrm{Cl}_{2}$ : $53.8 \mathrm{ppm}$, qui). Data are reported as follows: chemical shift $\delta / \mathrm{ppm}$, integration $\left({ }^{1} \mathrm{H}\right.$ only), multiplicity $(\mathrm{s}=$ singlet, $\mathrm{d}=$ doublet, $\mathrm{t}=$ triplet, $\mathrm{q}=$ quartet, $\mathrm{dd}=$ doublet of doublets, $\mathrm{m}=$ multiplet or combinations thereof; ${ }^{13} \mathrm{C}$ signals are singlets unless otherwise stated), coupling constants $\mathrm{J}$ in $\mathrm{Hz}$, assignment. ${ }^{19}$ F NMR spectra were recorded on the same Spectrometer.

High Resolution Mass Spectrometry (HRMS): All were recorded on Bruker micrOTOF II ESITOF by ESI or APCI. Measured values are reported to 4 decimal places of the calculated value. The calculated values are based on the most abundant isotope.

Chromatography: Analytical thin layer chromatography was performed using Qingdao Puke Parting Materials Co. silica gel plates (Silicagel 60 F254). Visualisation was by ultraviolet fluorescence $(\lambda=254 \mathrm{~nm})$ and/or staining with Phosphomolybdic acid or potassium permanganate $\left(\mathrm{KMnO}_{4}\right)$. Flash column chromatography was performed using 200-300 mesh silica gel. Optical rotations were measured with a polarimeter. [ $\alpha$ ]. D values are reported at a given temperature $\left({ }^{\circ} \mathrm{C}\right)$ in degrees $\mathrm{cm}^{2} \mathrm{~g}$ ${ }^{-1}$ with concentration in $\mathrm{mg} \mathrm{mL}^{-1}$.

Chiral HPLC: Enantiomeric excesses (ee) values were determined by chiral HPLC with chiral AS-H, AD-H, AZ-H, OJ-H columns with hexane and $i$-PrOH as solvents.

UV/Vis: Measurements were made on a Shimadzu RF-6000 Spectro Fluorophotometer.

Photoreactor: The photoreactors used in this research were bought from GeAo Chem (Figure S1, Figure S2 and Figure S3: white LEDs, light intensity $=61.4 \mathrm{mw} / \mathrm{cm}^{2}, 1 \mathrm{~W}$ for every light bulb; every two Schlenk tube was irradiated by 6 light bulbs from the side). The high pressure photoreactors used in this research were bought from Sichuan Zhiyan Technology Co., Ltd. (Figure S4: blue LEDs, $3 \mathrm{~W}$ for every light bulb; every bottle was irradiated by 2 light bulbs from the side).

Note: All photos in this material were taken by the first author Fu-Dong Lu.

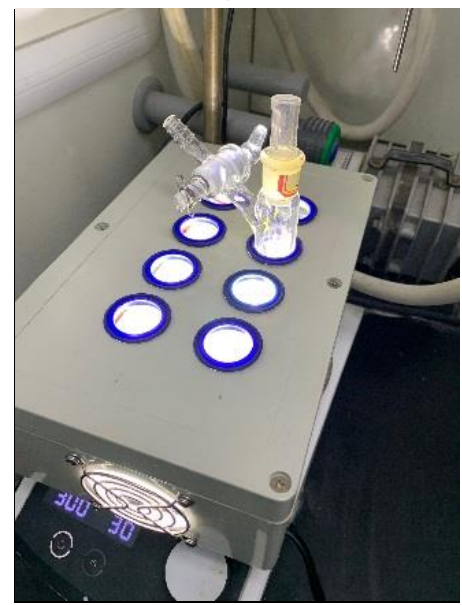

Figure S1. Photoreactor used in this research $(2 \times 3 \mathrm{~W}$ white LEDs $)$ 


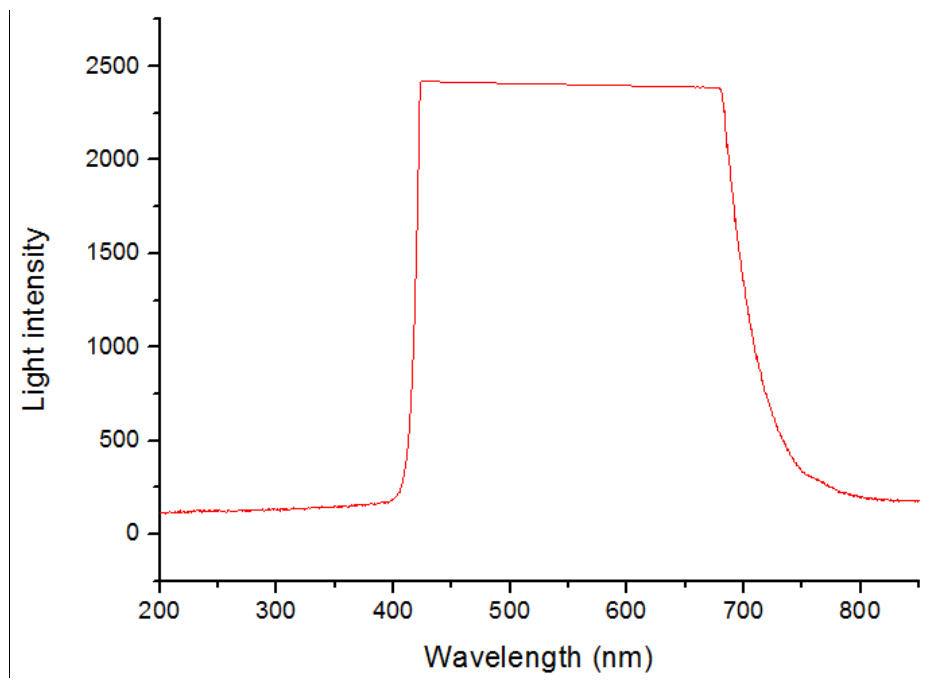

Figure S2. Spectrum of photoreactor ( 2 × 3 W white LEDs)

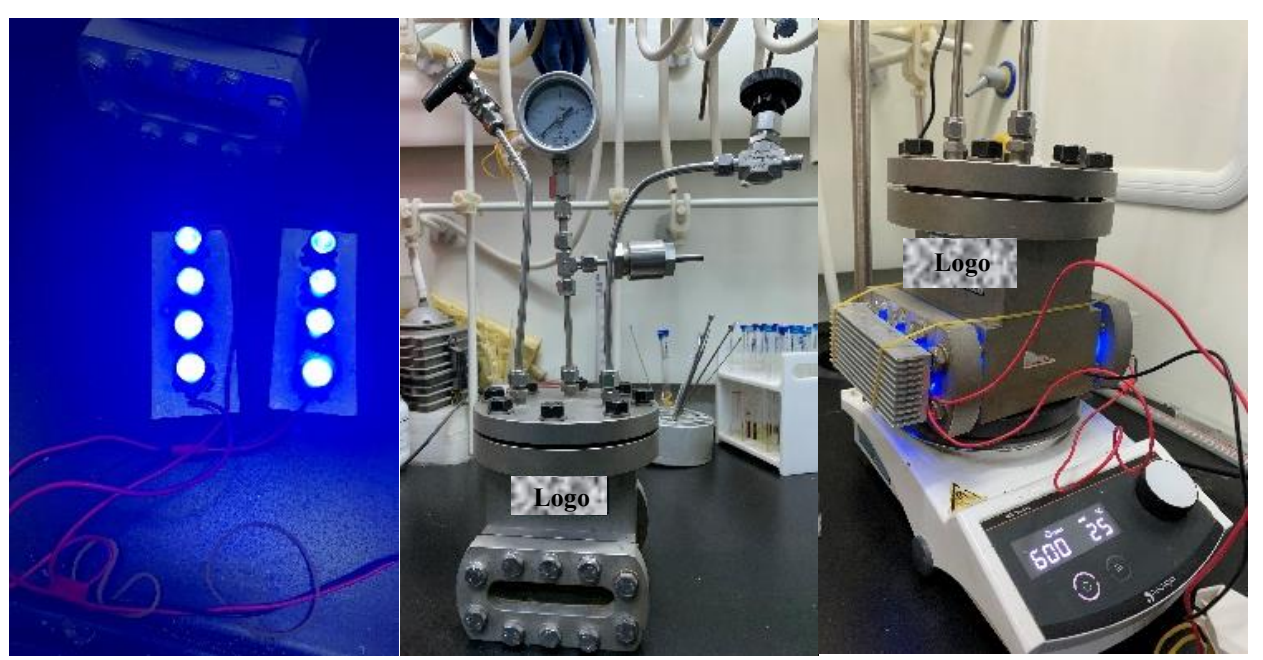

Figure S3. High pressure photoreactor used in this research ( $3 \mathrm{~W}$ blue LEDs, $\lambda_{\max }=455 \mathrm{~nm}$ )

\section{Preparation and Characterization of Materials}

Materials: Reagents, unless otherwise stated, were used as supplied from commercial sources without further purification. The 1,3-dienes, ${ }^{[1 \mathrm{a}]}$ 1,3-enynes ${ }^{[1 \mathrm{~b}]}$ and $\mathrm{N}$-(Acyloxy)phthalimides ${ }^{[2]}$ were prepared by following the literature report. Anhydrous solvent (THF, DCM, MeCN, DMF and DMSO) were taken from JC-Meyer solvent purification system. Anhydrous DCE were purchased from J\&K reagent company. Ligands L2-L5 were purchased from Daicel Chiral Technologies (China) Co., LTD. Ligands $\mathrm{L1}^{[3]}$, and PC1, PC2, PC3, PC5 ${ }^{[4]}$ were prepared according to literature methods. PC4 was purchased from Aladdin reagent company. TMSCN was purchased from Adamas reagent company. Note: The prepared 1,3-diene and 1,3-enyne should be stored in a Schlenk bottle, and stored at low temperature and protected from light under argon atmosphere, and used as soon as possible. If the storage time of 1,3-diene and 1,3-enyne is too long, it should be purified by flash column chromatography on silica gel to remove excess water and impurities before use. 


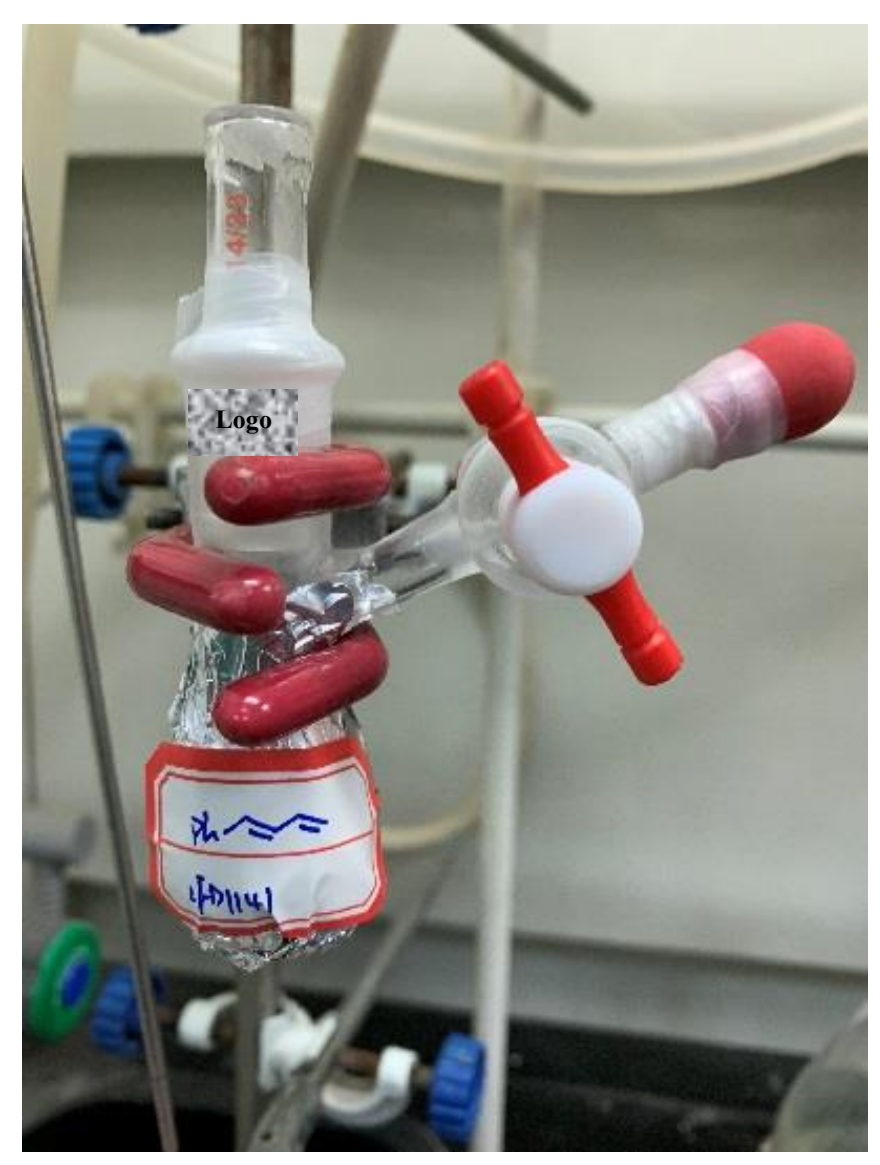

Figure S4. The storage of 1,3-dienes and 1,3-enynes

\section{General Procedure A:}

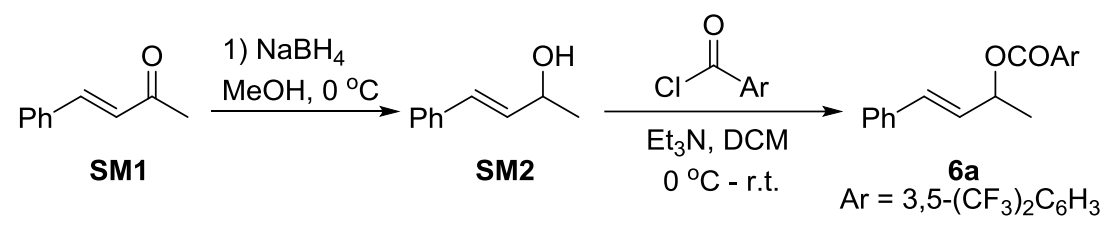

$\mathrm{NaBH}_{4}(0.91 \mathrm{~g}, 24 \mathrm{mmol}, 1.2$ equiv) was added to a stirred solution of benzylidene acetone (2.92 g, $20 \mathrm{mmol}, 1.0$ equiv) in $\mathrm{MeOH}(20 \mathrm{~mL})$ at $0{ }^{\circ} \mathrm{C}$, and the mixture was stirred at $0{ }^{\circ} \mathrm{C}$ for $30 \mathrm{~min}$. After that, the reaction was quenched by the addition of saturated aqueous $\mathrm{NH}_{4} \mathrm{Cl}$ and extracted with ethyl acetate. The organic layer was washed with brine, dried over $\mathrm{Na}_{2} \mathrm{SO}_{4}$, and concentrated under reduced pressure to obtain secondary allyl alcohol in the form of a yellow liquid in almost quantitative yield, which was used in the subsequent step without further purification.

To a solution of allyl alcohol (20 mmol, 1.0 equiv) in anhydrous $\mathrm{CH}_{2} \mathrm{Cl}_{2}(40 \mathrm{~mL})$ was added $\mathrm{Et}_{3} \mathrm{~N}$ (5.58 mL, $40 \mathrm{mmol}, 2.0$ equiv), followed by addition of 3,5-bis(trifluoromethyl)benzoyl chloride (5.44 $\mathrm{mL}, 30 \mathrm{mmol}, 1.5$ equiv) at $0{ }^{\circ} \mathrm{C}$. The reaction mixture was stirred at room temperature for overnight. After completion of the reaction (TLC), the reaction was diluted with $\mathrm{Et}_{2} \mathrm{O}(100 \mathrm{~mL})$ and washed with $\mathrm{H}_{2} \mathrm{O}$. The organic layer was washed with brine, dried over $\mathrm{Na}_{2} \mathrm{SO}_{4}$. The product was purified by flash column chromatography on silica gel using (pet. ether/ethyl acetate) as eluent to afford allyl esters 6a as a light-yellow solid. ${ }^{[5]}$ 


\section{(E)-4-Phenylbut-3-en-2-yl 3,5-bis(trifluoromethyl)benzoate (6a)}

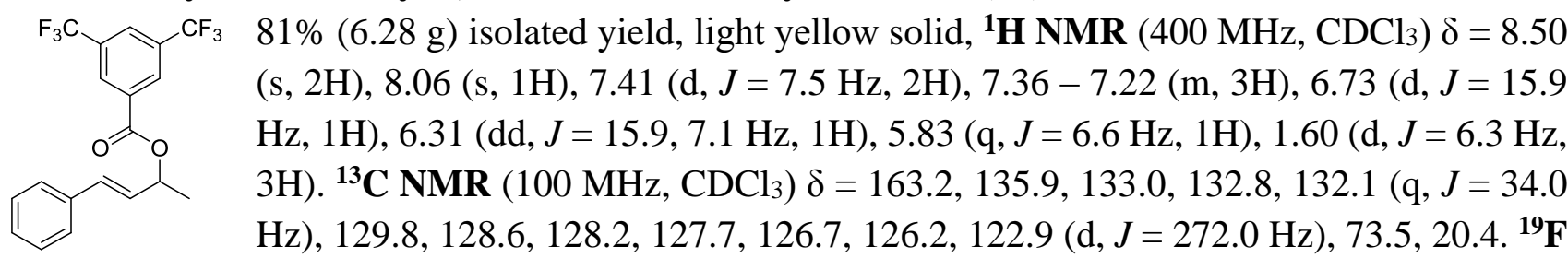

NMR $\left(376 \mathrm{MHz}, \mathrm{CDCl}_{3}\right) \delta=-62.93$. HRMS (ESI) for $\mathrm{C}_{19} \mathrm{H}_{14} \mathrm{~F}_{6} \mathrm{NaO}_{2}[\mathrm{M}+\mathrm{Na}]^{+}$: calcd 411.0790, found 411.0785 .

\section{General Procedure B:}

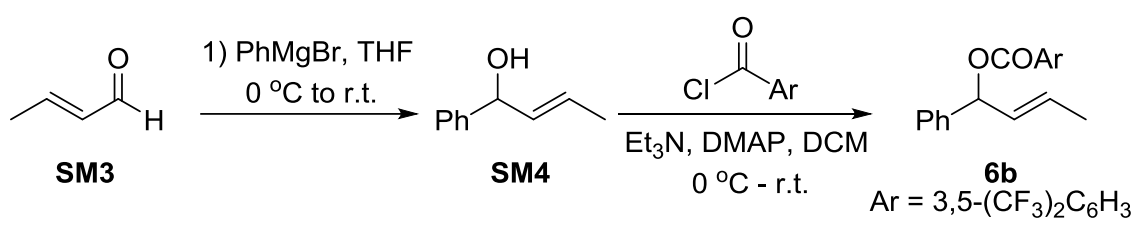

$\mathrm{PhMgBr}$ (1.0 M in THF, $24 \mathrm{~mL}, 24 \mathrm{mmol}, 1.2$ equiv) was added slowly to a stirred solution of benzylidene acetone $\left(1.40 \mathrm{~g}, 20 \mathrm{mmol}, 1.0\right.$ equiv) in THF $(20 \mathrm{~mL})$ at $0{ }^{\circ} \mathrm{C}$, and the mixture was stirred at room temperature for overnight. The reaction was quenched by the addition of ice water and extracted with ethyl acetate. The organic layer was washed with brine, dried over $\mathrm{Na}_{2} \mathrm{SO}_{4}$, and concentrated under reduced pressure to obtain secondary allyl alcohol in the form of a yellow liquid in excellent yield, which was used in the subsequent step without further purification.

To a solution of allyl alcohol (20 mmol, 1.0 equiv) in anhydrous $\mathrm{CH}_{2} \mathrm{Cl}_{2}(40 \mathrm{~mL})$ was added $\mathrm{Et}_{3} \mathrm{~N}$ (5.58 mL, $40 \mathrm{mmol}, 2.0$ equiv) and DMAP (200 mg, cat.), followed by addition of 3,5bis(trifluoromethyl)benzoyl chloride $\left(5.44 \mathrm{~mL}, 30 \mathrm{mmol}, 1.5\right.$ equiv) at $0{ }^{\circ} \mathrm{C}$. The reaction mixture was stirred at room temperature for overnight. After completion of the reaction (TLC), the reaction was quenched by saturated aqueous $\mathrm{NcHCO}_{3}$ and extracted with $\mathrm{Et}_{2} \mathrm{O}$. The organic layer was washed with brine, dried over $\mathrm{Na}_{2} \mathrm{SO}_{4}$. The product was purified by flash column chromatography on silica gel using (pet. ether/ethyl acetate) as eluent to afford allyl esters $\mathbf{6 b}$ as a light-yellow oil.

\section{(E)-1-Phenylbut-2-en-1-yl 3,5-bis(trifluoromethyl)benzoate (6b)}

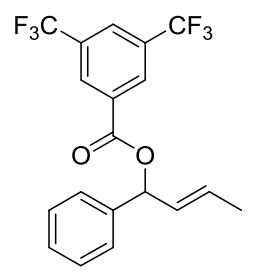

$60 \%$ (4.21 g) isolated yield, light yellow oil, ${ }^{1} \mathbf{H}$ NMR $\left(400 \mathrm{MHz}, \mathrm{CD}_{2} \mathrm{Cl}_{2}\right) \delta=8.42$ $(\mathrm{s}, 2 \mathrm{H}), 7.97(\mathrm{~s}, 1 \mathrm{H}), 7.33-7.26(\mathrm{~m}, 2 \mathrm{H}), 7.23-7.10(\mathrm{~m}, 3 \mathrm{H}), 6.61(\mathrm{~d}, J=15.9 \mathrm{~Hz}$, $1 \mathrm{H}), 6.23(\mathrm{dd}, J=15.9,7.0 \mathrm{~Hz}, 1 \mathrm{H}), 5.74-5.68(\mathrm{~m}, 1 \mathrm{H}), 1.47(\mathrm{~d}, J=6.5 \mathrm{~Hz}, 3 \mathrm{H})$. ${ }^{13} \mathrm{C}$ NMR $\left(100 \mathrm{MHz}, \mathrm{CD}_{2} \mathrm{Cl}_{2}\right) \delta=163.5,136.5,133.4,132.9,132.3(\mathrm{q}, J=34.0 \mathrm{~Hz})$, $130.2(\mathrm{~m}), 129.1,128.6,128.4,127.0,126.7(\mathrm{~m}), 123.5(\mathrm{q}, J=271.0 \mathrm{~Hz}), 121.9,73.9$, 20.6. ${ }^{19} \mathbf{F}$ NMR $\left(376 \mathrm{MHz}, \mathrm{CD}_{2} \mathrm{Cl}_{2}\right) \delta=-62.93$. HRMS (ESI) for $\mathrm{C}_{19} \mathrm{H}_{14} \mathrm{~F}_{6} \mathrm{NaO}_{2}[\mathrm{M}+\mathrm{Na}]^{+}$: calcd 411.0790, found 411.0792.

\section{References}

[1] (a) Takaya, J.; Sasano, K.; Iwasawa, N. Org. Lett. 2011, 13, 1698. (b) Wang, F.; Wang, D.-H.; Zhou, Y.; Liang, L.; 
Lu, R.-H.; Chen, P.-H.; Lin, Z.-Y.; Liu, G. Angew. Chem. Int. Ed. 2018, 57, 7140.

[2] Wang, D.-H.; Zhu, N.; Chen, P.-H.; Lin, Z.-Y.; Liu, G. J. Am. Chem. Soc. 2017, 139, 15632.

[3] Liu, C.; Yi, J.-C.; Zheng, Z.-B.; Tang, Y.; Dai, L.-X.; You S.-L. Angew. Chem. Int. Ed. 2016, 55, 751.

[4] Pan, X.-C.; Fang, C.; Fantin, M.; Malhotra, N.; So, W. Y.; Peteanu, L. A.; Isse, A. A.; Gennaro, A.; Liu, P.; Matyjaszewski, K. J. Am. Chem. Soc. 2016, 138, 2411.

[5] Rackl, D.; Kais, V.; Kreitmeier, P.; Reiser, O. Beilstein J. Org. Chem. 2014, 10, 2157. 


\section{Details for Condition Optimization}

\subsection{Table S1. The Effect of Copper Salts ${ }^{\mathrm{a}}$}
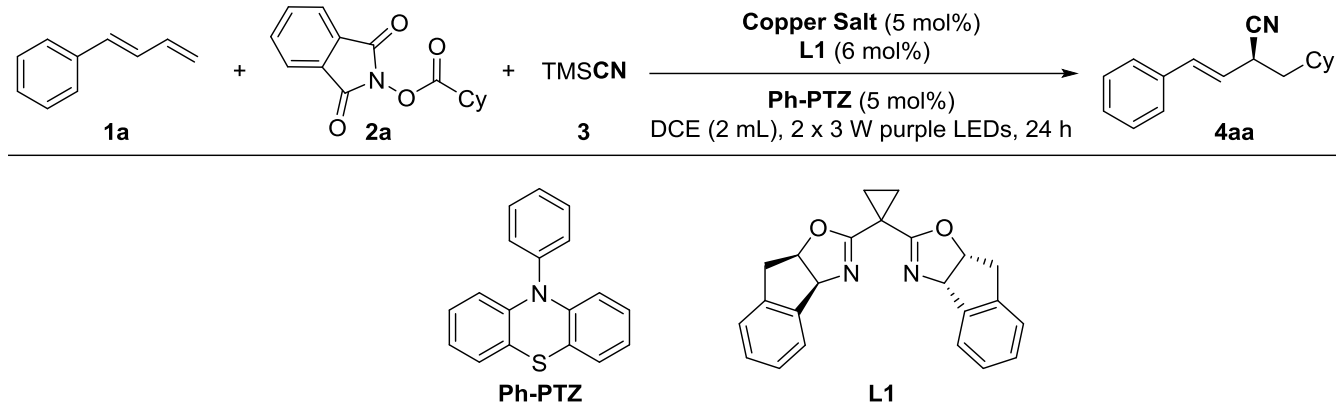

L1

\begin{tabular}{cccc}
\hline Entry & Copper Salts & Yield $/ \%^{\mathrm{b}}$ & $\mathrm{Ee} / \%^{\mathrm{c}}$ \\
\hline 1 & $\mathrm{Cu}\left(\mathrm{CH}_{3} \mathrm{CN}\right)_{4} \mathrm{BF}_{4}$ & 39 & 91 \\
2 & $\mathrm{Cu}\left(\mathrm{CH}_{3} \mathrm{CN}\right)_{4} \mathrm{PF}_{6}$ & 30 & 88 \\
3 & $\mathrm{CuOAc}$ & 38 & 82 \\
4 & $\mathrm{CuCN}$ & 41 & 89 \\
5 & $\mathrm{Cu}(\mathrm{OTf})_{2}$ & Trace & - \\
\hline
\end{tabular}

a 1 (a 0.4 mmol, 2.0 equiv), 2 a ( $0.2 \mathrm{mmol}, 1.0$ equiv), TMSCN (0.3 mmol, 1.5 equiv), copper salt ( $0.01 \mathrm{mmol}, 5$ mol\%), chiral ligand $\mathbf{L 1}(0.012 \mathrm{mmol}, 6 \mathrm{~mol} \%)$ and organo-photocatalyst Ph-PTZ (0.01 mmol, $5 \mathrm{~mol} \%)$ in 2.0 $\mathrm{mL}$ of DCE for $24 \mathrm{~h}$ under the irradiation of $2 \times 3 \mathrm{~W}$ purple LEDs. ${ }^{b} \mathrm{NMR}$ yield determined using 1,3,5trimethoxybenzene as an internal standard. ${ }^{\mathrm{c}}$ Determined by chiral HPLC.

\subsection{Table S2. The Effect of Chiral Ligands $\mathrm{s}^{\mathrm{a}}$}

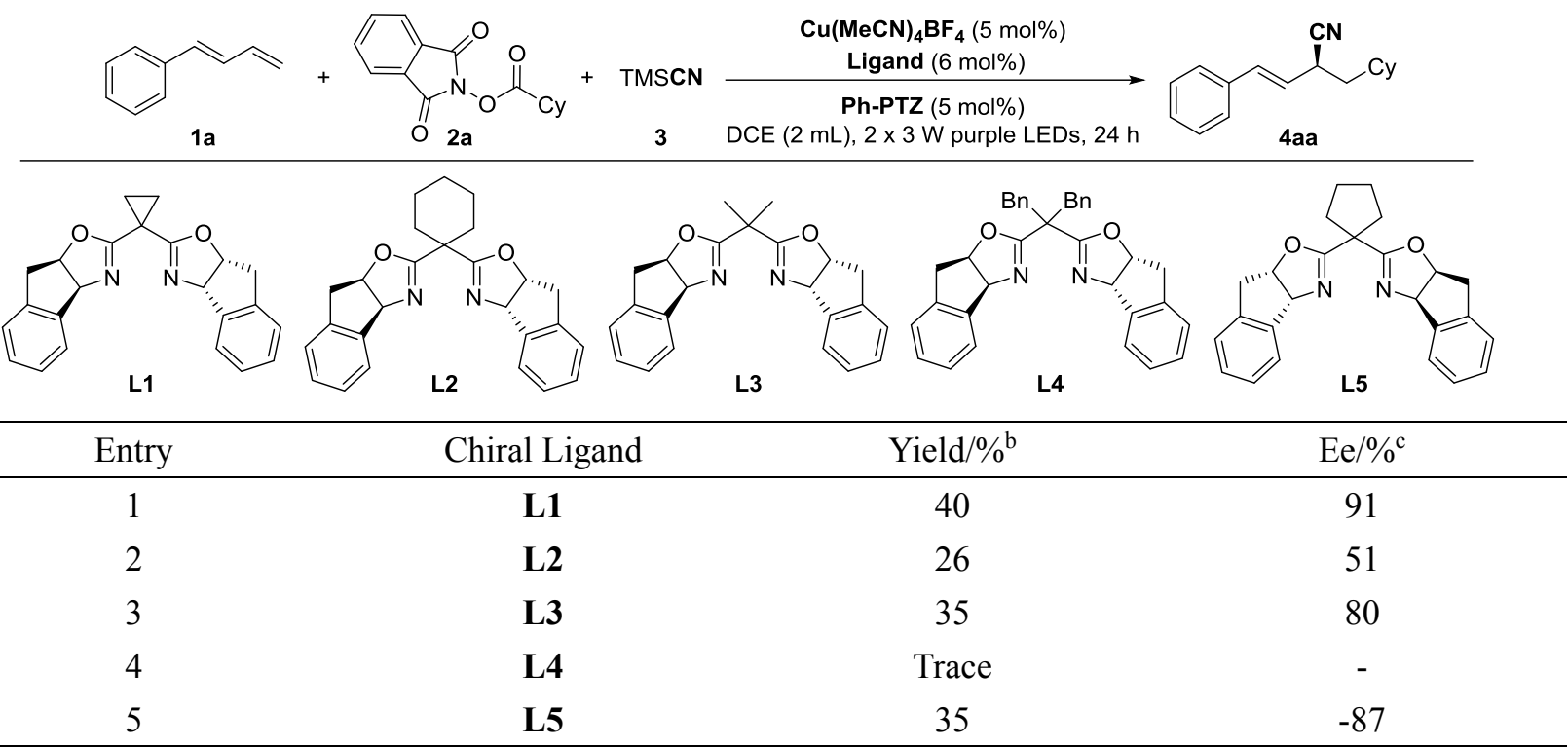

${ }^{a} \mathbf{1 a}\left(0.4 \mathrm{mmol}, 2.0\right.$ equiv), 2a (0.2 mmol, 1.0 equiv), TMSCN (0.3 mmol, 1.5 equiv), $\mathbf{C u}(\mathbf{M e C N})_{4} \mathbf{B F}_{4}(0.01$ mmol, $5 \mathrm{~mol} \%)$, chiral ligand (0.012 mmol, $6 \mathrm{~mol} \%$ ) and organo-photocatalyst Ph-PTZ (0.01 mmol, $5 \mathrm{~mol} \%)$ in $2.0 \mathrm{~mL}$ of DCE for $24 \mathrm{~h}$ under the irradiation of $2 \times 3 \mathrm{~W}$ purple LEDs. ${ }^{b} \mathrm{NMR}$ yield determined using 1,3,5trimethoxybenzene as an internal standard. ${ }^{\mathrm{c}}$ Determined by chiral HPLC. 


\subsection{Table S3. The Effect of Solvents ${ }^{\mathrm{a}}$}

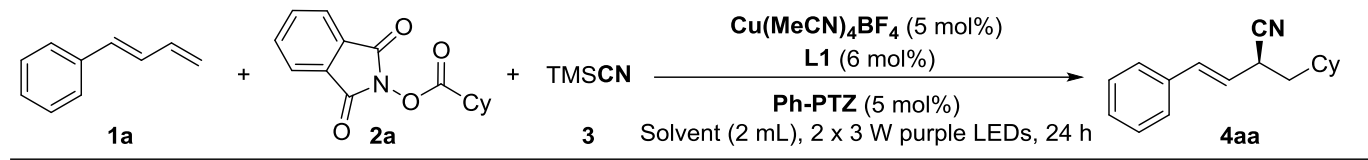

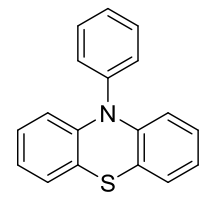

Ph-PTZ

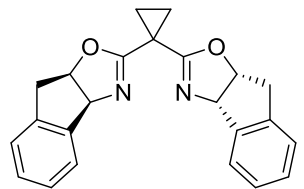

L1

\begin{tabular}{cccc}
\hline Entry & Solvent & Yield $/ \%^{\mathrm{b}}$ & $\mathrm{Ee} / \%^{\mathrm{c}}$ \\
\hline 1 & $\mathrm{THF}$ & Trace & 91 \\
2 & $\mathrm{DCM}$ & 20 & 90 \\
3 & $\mathrm{MeCN}$ & 22 & 85 \\
4 & $\mathrm{DMF}$ & 21 & 85 \\
5 & $\mathrm{DCE}$ & 40 & 91 \\
6 & $\mathrm{CHCl}$ & $\mathrm{NR}$ & - \\
7 & $\mathrm{DMSO}$ & 20 & 82 \\
\hline
\end{tabular}

${ }^{\mathrm{a}} \mathbf{1 a}\left(0.4 \mathrm{mmol}, 2.0\right.$ equiv), $\mathbf{2 a}\left(0.2 \mathrm{mmol}, 1.0\right.$ equiv), TMSCN (0.3 mmol, 1.5 equiv), $\mathbf{C u}(\mathbf{M e C N})_{4} \mathbf{B F}_{4}(0.01$ mmol, $5 \mathrm{~mol} \%)$, chiral ligand L1 $(0.012 \mathrm{mmol}, 6 \mathrm{~mol} \%)$ and organo-photocatalyst Ph-PTZ (0.01 mmol, 5 mol\%) in $2.0 \mathrm{~mL}$ of solvent for $24 \mathrm{~h}$ under the irradiation of $2 \times 3 \mathrm{~W}$ purple LEDs. ${ }^{b} \mathrm{NMR}$ yield determined using 1,3,5-trimethoxybenzene as an internal standard. ${ }^{\mathrm{c}}$ Determined by chiral HPLC.

\subsection{Table S4. The Effect of TMSCN's Equivalent ${ }^{\mathrm{a}}$}
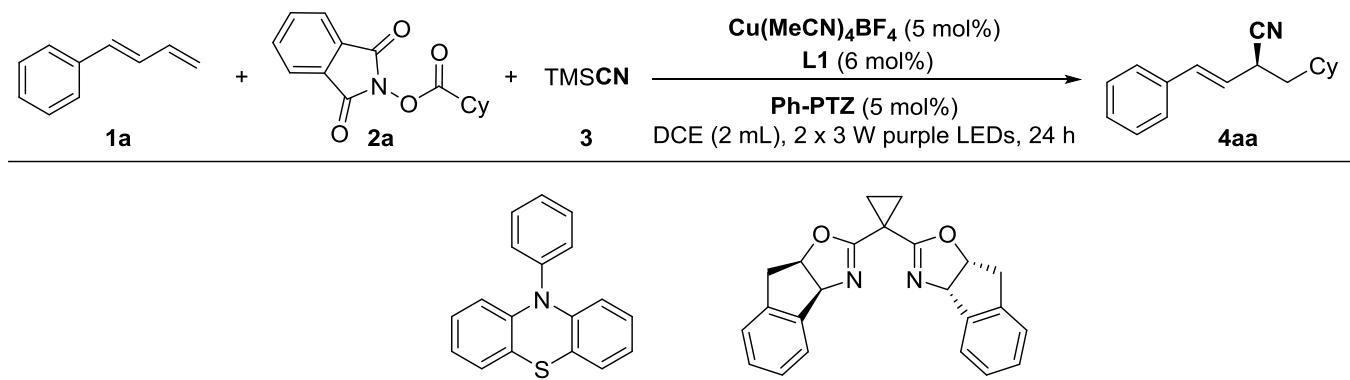

Ph-PTZ

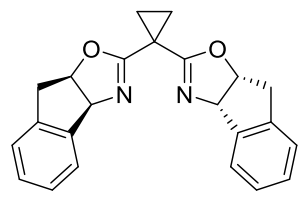

L1

\begin{tabular}{cccc}
\hline Entry & Equivalent of TMSCN $(\mathrm{x})$ & Yield $/ \%^{\mathrm{b}}$ & $\mathrm{Ee} / \%^{\mathrm{c}}$ \\
\hline 1 & 1.0 & 32 & 91 \\
2 & 1.5 & 40 & 91 \\
3 & 3.0 & 45 & 91 \\
\hline
\end{tabular}

${ }^{a} \mathbf{1 a}\left(0.4 \mathrm{mmol}, 2.0\right.$ equiv), $\mathbf{2 a}\left(0.2 \mathrm{mmol}, 1.0\right.$ equiv), TMSCN (x equiv), $\mathbf{C u}(\mathbf{M e C N})_{4} \mathbf{B F}_{4}(0.01 \mathrm{mmol}, 5 \mathrm{~mol} \%)$, chiral ligand $\mathbf{L 1}(0.012 \mathrm{mmol}, 6 \mathrm{~mol} \%)$ and organo-photocatalyst Ph-PTZ (0.01 mmol, $5 \mathrm{~mol} \%)$ in $2.0 \mathrm{~mL}$ of

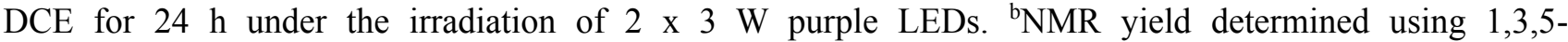
trimethoxybenzene as an internal standard. ${ }^{\mathrm{c}}$ Determined by chiral HPLC. 


\subsection{Table S5. The Effect of 1,3-Diene's Equivalent ${ }^{\mathrm{a}}$}

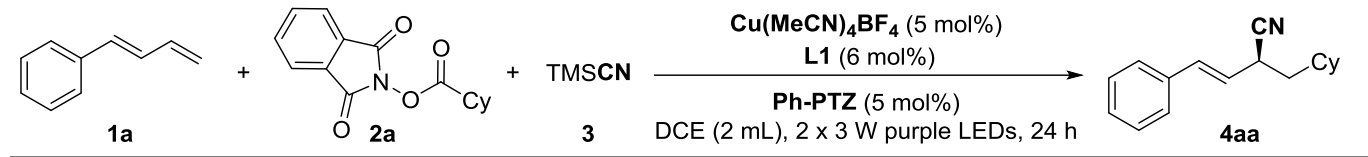

\begin{tabular}{cccc} 
\\
Entry & Equivalent of $\mathbf{1 a}(\mathrm{x})$ & $\mathrm{Eield} / \%^{\mathrm{b}}$ & \\
\hline 1 & 1.0 & 16 & 91 \\
2 & 2.0 & 45 & 91 \\
3 & 3.0 & 54 & ${ }^{\mathrm{c}}$ \\
\hline
\end{tabular}

${ }^{a} \mathbf{1 a}$ (x equiv), 2a (0.2 mmol, 1.0 equiv), TMSCN (0.6 mmol, 3.0 equiv), $\mathbf{C u}(\mathbf{M e C N})_{4} \mathbf{B F}_{4}(0.01 \mathrm{mmol}, 5 \mathrm{~mol} \%)$, chiral ligand $\mathbf{L 1}(0.012 \mathrm{mmol}, 6 \mathrm{~mol} \%)$ and organo-photocatalyst Ph-PTZ $(0.01 \mathrm{mmol}, 5 \mathrm{~mol} \%)$ in $2.0 \mathrm{~mL}$ of DCE for $24 \mathrm{~h}$ under the irradiation of $2 \times 3 \mathrm{~W}$ purple LEDs. ${ }^{\text {bNMR }}$ yield determined using 1,3,5trimethoxybenzene as an internal standard. ${ }^{\mathrm{c}}$ Determined by chiral HPLC.

\subsection{Table S6. The Effect of Photocatalyst ${ }^{\mathrm{a}}$}
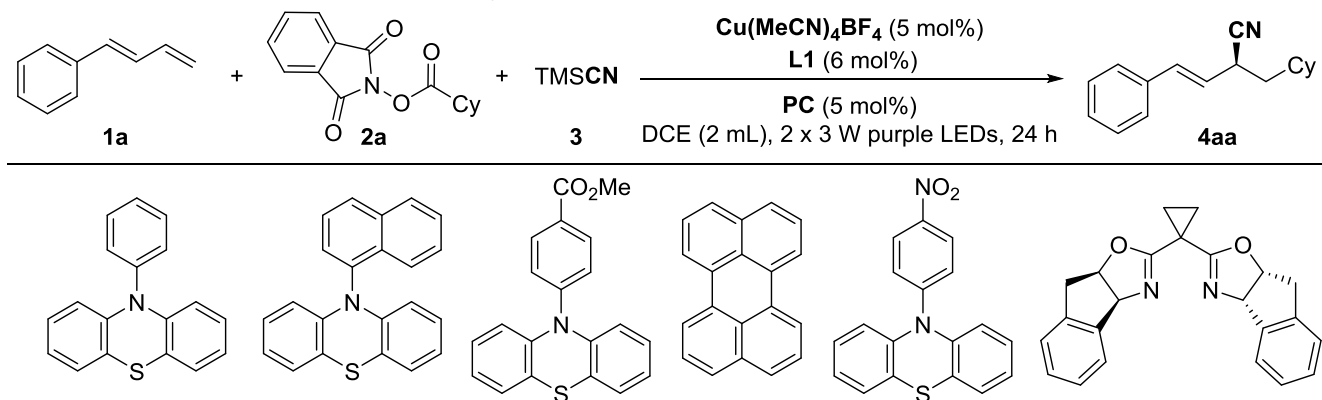

PC2

PC3

PC4
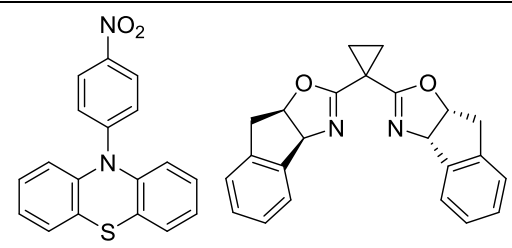

\begin{tabular}{cccc}
\hline Entry & Photocatalyst & Yield $/ \%^{\mathrm{b}}$ & $\mathrm{Ee} / \%^{\mathrm{c}}$ \\
\hline 1 & PC1 & 52 & 91 \\
2 & PC2 & 45 & 91 \\
3 & PC3 & 40 & 91 \\
$4^{\mathrm{d}}$ & PC4 (Perylene) & 55 & 91 \\
5 & PC5 & NR & - \\
\hline
\end{tabular}

${ }^{\mathrm{a}} \mathbf{1 a}\left(0.6 \mathrm{mmol}, 3.0\right.$ equiv), 2a (0.2 mmol, 1.0 equiv), TMSCN (0.6 mmol, 3.0 equiv), $\mathbf{C u}(\mathbf{M e C N})_{4} \mathbf{B F}_{4}(0.01$ mmol, $5 \mathrm{~mol} \%)$, chiral ligand $\mathbf{L 1}(0.012 \mathrm{mmol}, 6 \mathrm{~mol} \%)$ and organo-photocatalyst $(0.01 \mathrm{mmol}, 5 \mathrm{~mol} \%)$ in 2.0 $\mathrm{mL}$ of DCE for $24 \mathrm{~h}$ under the irradiation of $2 \times 3 \mathrm{~W}$ purple LEDs. ${ }^{b} \mathrm{NMR}$ yield determined using 1,3,5trimethoxybenzene as an internal standard. ${ }^{\mathrm{c}}$ Determined by chiral HPLC. ${ }^{\mathrm{d}} 2$ x $3 \mathrm{~W}$ white LEDs, $36 \mathrm{~h}$. 


\subsection{Table S7. The Effect of Concentration ${ }^{\mathrm{a}}$}
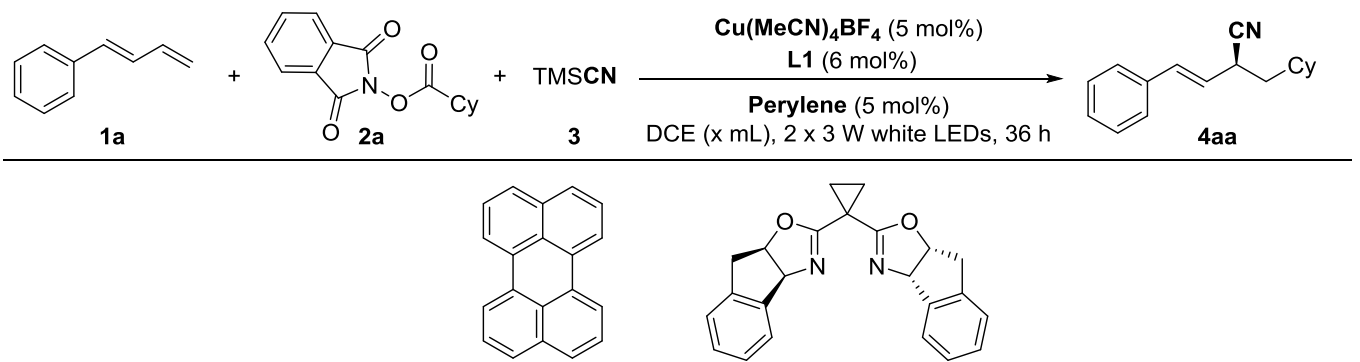

PC4 (Perylene)

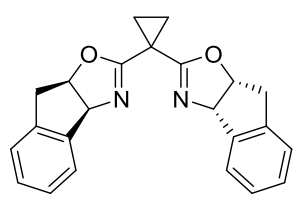

L1

\begin{tabular}{cccc}
\hline Entry & $\mathrm{X}(\mathrm{mL})$ & Yield $/ \%^{\mathrm{b}}$ & $\mathrm{Ee} / \%^{\mathrm{c}}$ \\
\hline 1 & 0.5 & 70 & 92 \\
2 & 1.0 & 58 & 91 \\
3 & 2.0 & 55 & 90 \\
4 & 4.0 & 37 & 90 \\
\hline
\end{tabular}

${ }^{\mathrm{a}} \mathbf{1 a}\left(0.6 \mathrm{mmol}, 3\right.$ equiv), $\mathbf{2 a}\left(0.2 \mathrm{mmol}, 1.0\right.$ equiv), TMSCN ( $0.6 \mathrm{mmol}, 3$ equiv), $\mathbf{C u}(\mathbf{M e C N})_{4} \mathbf{B F}_{4}(0.01 \mathrm{mmol}$, $5 \mathrm{~mol} \%)$, chiral ligand L1 (0.012 mmol, $6 \mathrm{~mol} \%$ ) and Perylene (0.01 mmol, $5 \mathrm{~mol} \%)$ in x mL of DCE for $36 \mathrm{~h}$ under the irradiation of $2 \times 3 \mathrm{~W}$ white LEDs. ${ }^{b} \mathrm{NMR}$ yield determined using 1,3,5-trimethoxybenzene as an internal standard. ${ }^{\mathrm{c}}$ Determined by chiral HPLC.

\subsection{Table S8. The Effect of Light Source ${ }^{a}$}
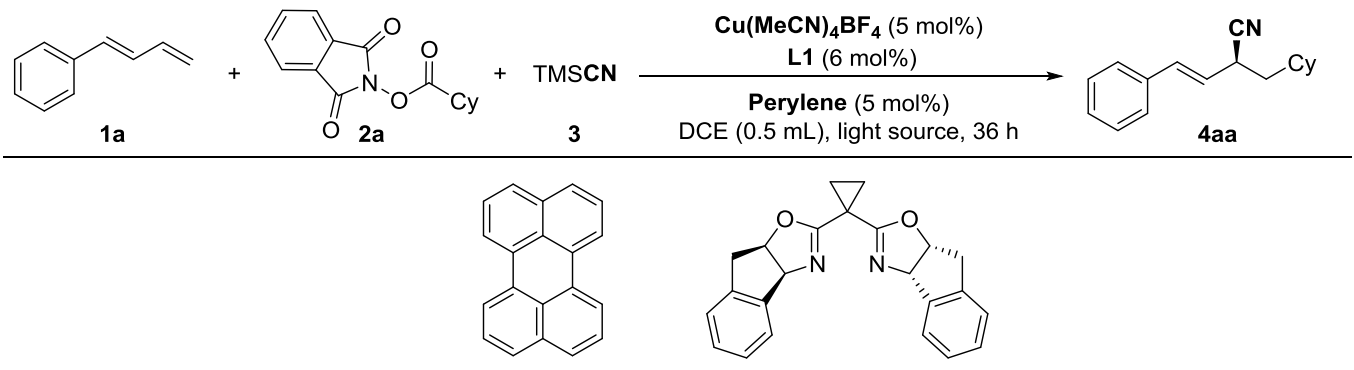

PC4 (Perylene)

L1

\begin{tabular}{cccc}
\hline Entry & Light source & Yield $/ \%^{\mathrm{b}}$ & Ee/\% ${ }^{\mathrm{c}}$ \\
\hline 1 & $2 \times 3$ W blue LEDs & 61 & 91 \\
2 & $2 \times 3$ W white LEDs & 71 & 92 \\
3 & 7 W blue LED & 15 & 90 \\
4 & 7 W white LED & NR & - \\
\hline
\end{tabular}

${ }^{\mathrm{a}} \mathbf{1 a}\left(0.6 \mathrm{mmol}, 3\right.$ equiv), $\mathbf{2 a}\left(0.2 \mathrm{mmol}, 1.0\right.$ equiv), TMSCN ( $0.6 \mathrm{mmol}, 3.0$ equiv), $\mathbf{C u}(\mathbf{M e C N})_{4} \mathbf{B F}_{4}(0.01 \mathrm{mmol}$, $5 \mathrm{~mol} \%)$, chiral ligand $\mathbf{L 1}(0.012 \mathrm{mmol}, 6 \mathrm{~mol} \%)$ and Perylene $(0.01 \mathrm{mmol}, 5 \mathrm{~mol} \%)$ in $0.5 \mathrm{~mL}$ of DCE for 36 $\mathrm{h}$ under the irradiation of LEDs. ${ }^{\mathrm{b}} \mathrm{NMR}$ yield determined using 1,3,5-trimethoxybenzene as an internal standard. ${ }^{\mathrm{c}}$ Determined by chiral HPLC. 


\subsection{Table S9. Control Experiments ${ }^{\mathrm{a}}$}
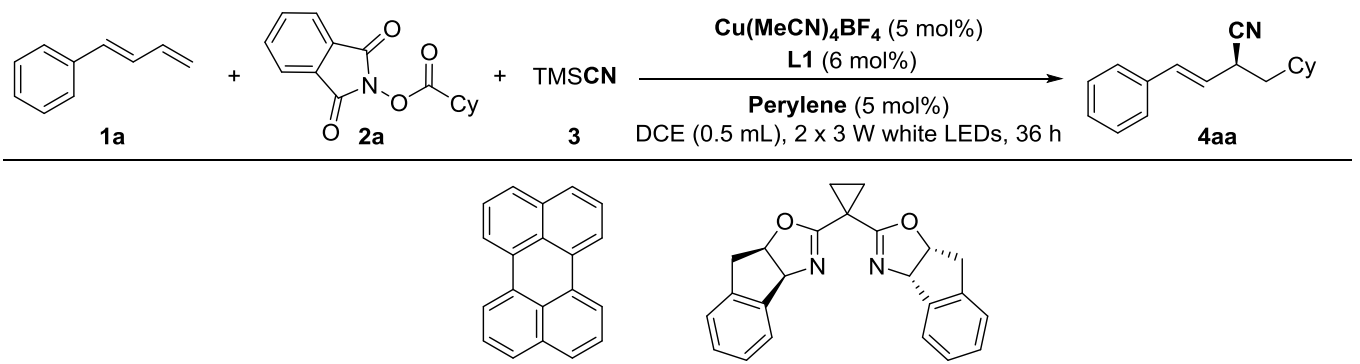

PC4 (Perylene)

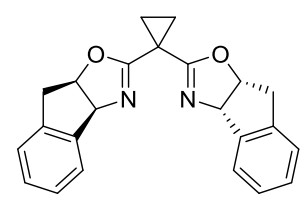

L1

\begin{tabular}{cccc}
\hline Entry & Variation & Yield $/ \%^{\mathrm{b}}$ & Ee $/ \%$ \\
\hline 1 & No light & NR & ND \\
2 & No Perylene & NR & ND \\
3 & No Cu/L & NR & ND
\end{tabular}

${ }^{\mathrm{a}} \mathbf{1 a}\left(0.6 \mathrm{mmol}, 3\right.$ equiv), $\mathbf{2 a}\left(0.2 \mathrm{mmol}, 1.0\right.$ equiv), TMSCN ( $0.6 \mathrm{mmol}, 3.0$ equiv), $\mathbf{C u}(\mathbf{M e C N})_{\mathbf{4}} \mathbf{B F}_{\mathbf{4}}(0.01 \mathrm{mmol}$, $5 \mathrm{~mol} \%)$, chiral ligand $\mathbf{L 1}(0.012 \mathrm{mmol}, 6 \mathrm{~mol} \%)$ and Perylene $(0.01 \mathrm{mmol}, 5 \mathrm{~mol} \%)$ in $0.5 \mathrm{~mL}$ of DCE for 36 $\mathrm{h}$ under the irradiation of $2 \times 3 \mathrm{~W}$ white LEDs. ${ }^{\mathrm{b}} \mathrm{NMR}$ yield determined using 1,3,5-trimethoxybenzene as an internal standard. NR: no reaction. ND: not determined. 


\section{General Procedures and Characterization Data of Products}

\subsection{General Procedures}

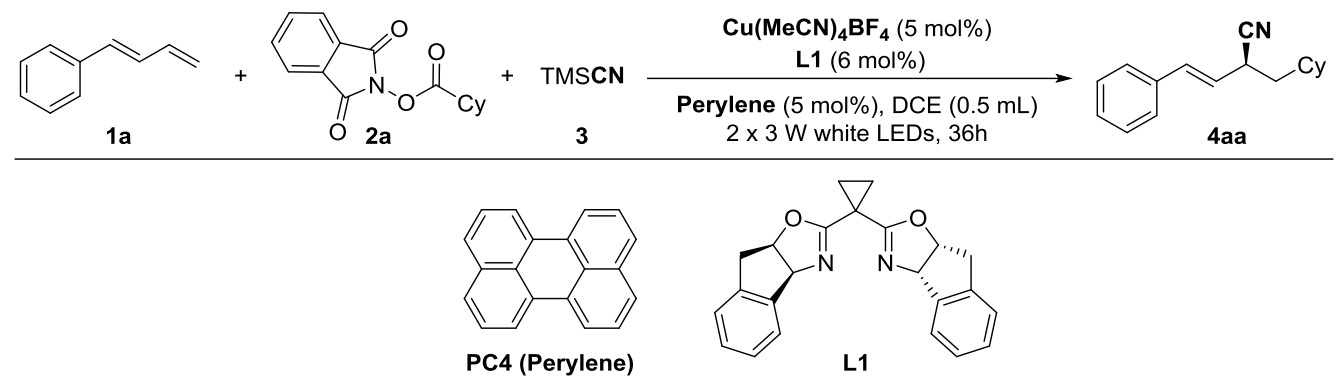

General procedure $\mathbf{C}$ (with product 4aa as an example): In an argon-filled glove box, a flamedried $10 \mathrm{ml}$ Schlenk tube equipped with a magnetic stirrer bar and rubber plug was charged sequentially with $\mathbf{C u}\left(\mathbf{C H}_{3} \mathbf{C N}\right)_{4} \mathbf{B F}_{4}(3.15 \mathrm{mg}, 0.01 \mathrm{mmol})$ and $\mathbf{L 1}$ (4.28 $\left.\mathrm{mg}, 0.012 \mathrm{mmol}\right)$, followed by the addition of DCE $(0.5 \mathrm{~mL})$. The mixture was then stirred at room temperature for 30 minutes. $N$-(Acyloxy)phthalimides 2a $(54.6 \mathrm{mg}, 0.20 \mathrm{mmol})$, organo-photocatalyst Perylene $(2.52 \mathrm{mg}, 0.01$ mmol) and TMSCN $(59.5 \mathrm{mg}, 76 \mu \mathrm{L}, 0.6 \mathrm{mmol})$ were added to the resulting mixture. The vial was closed and the Schlenk tube was removed from the glove box. After that, 1,3-diene 1a $(78.1 \mathrm{mg}, 84 \mu \mathrm{L}$, $0.6 \mathrm{mmol}$ ) was added into the mixture through microinjector. At last, the mixture was stirred at a distance of $\sim 1 \mathrm{~cm}$ from a $2 \times 3 \mathrm{~W}$ white LEDs at $29^{\circ} \mathrm{C}$ about $36 \mathrm{~h}$ until the reaction was completed, as monitored by TLC analysis. The product was purified by flash column chromatography on silica gel to afford the desired product.

The reaction for 4aa-4ai, $4 \mathbf{b d}-\mathbf{4 l d}, \mathbf{4 n d}, \mathbf{4 p d}$ and $\mathbf{4 q d}$ were performed according to the general procedure $\mathrm{C}$. The reaction for $\mathbf{4 m d}$ and $\mathbf{4 o d}$ were prepared according to the general procedure $\mathrm{C}$ by replacing perylene and light with $1 \mathrm{~mol} \% f a c-\operatorname{Ir}(\text { ppy })_{3}$ and $2 \times 3 \mathrm{~W}$ blue LEDs respectively. The reaction for 4aj was prepared according to the general procedure $\mathrm{C}$ by replacing perylene, $0.5 \mathrm{~mL} \mathrm{DCE}$ and light with $5 \mathrm{~mol} \%$ Ph-PTZ, $2 \mathrm{~mL}$ DCE and $2 \times 3 \mathrm{~W}$ purple LEDs respectively. It is worth noting that the polarity of the Perylene and the products 4 in the reactions are very close. In the separation process, the Perylene needs to be separated with petroleum ether and dichloromethane (200/1) first, and then the product is separated with petroleum ether and ethyl acetate (100/1).

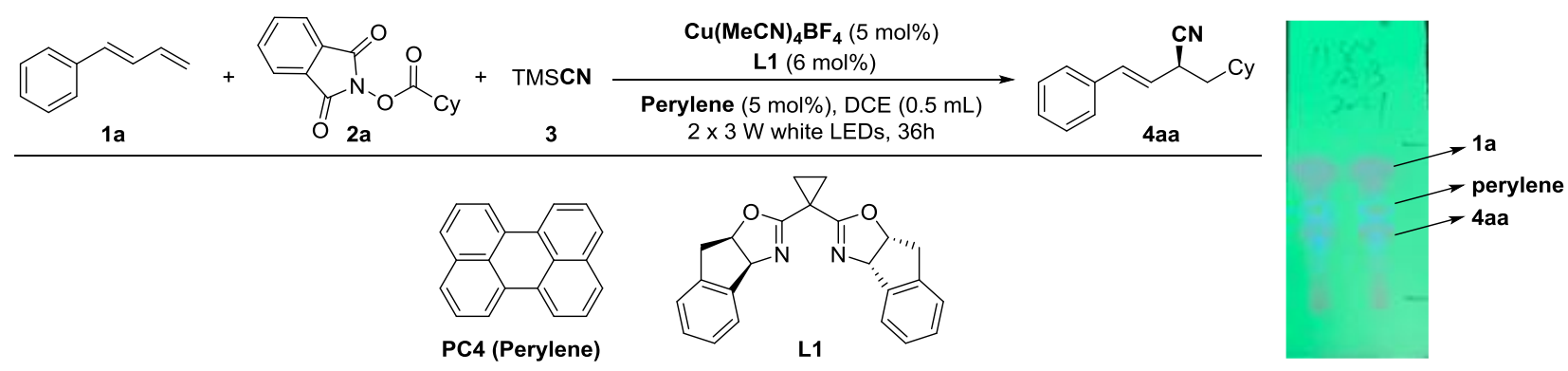

Figure S5. Photographic guide for TLC plates of the asymmetric carbocyanation reaction 


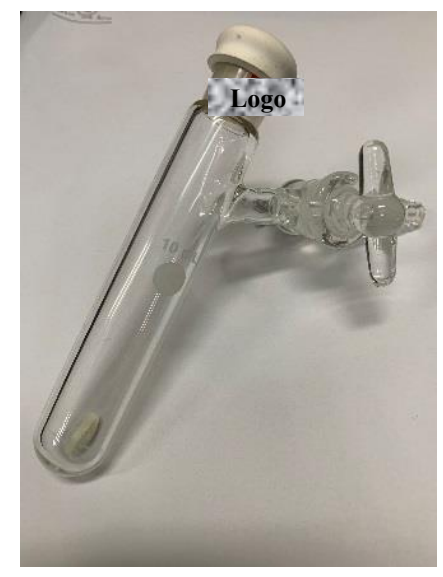

Figure S6. Schlenk tube equipped with a magnetic stirrer bar and rubber plug

Note: The racemic samples were prepared according to the general procedure A by replacing the chiral ligand $\mathbf{L 1}$ with no-chiral ligand $\mathbf{L 6}$.

\subsection{Characterization Data of Products}

\section{$(S, E)$-2-(Cyclohexylmethyl)-4-phenylbut-3-enenitrile (4aa)}

$\mathrm{CN}_{\mathrm{N}} 66 \%(31.3 \mathrm{mg})$ isolated yield, light yellow oil, $[\alpha]_{\mathrm{D}}{ }^{25}=6.66\left(\mathrm{c}=1.04\right.$ in $\left.\mathrm{CHCl}_{3}\right) ; 91 \%$ ee, determined by HPLC analysis (Chiralpak AS-H column, hexane/i-PrOH, 85:15 $\mathrm{v} / \mathrm{v}$, flow rate $0.5 \mathrm{~mL} / \mathrm{min}, \lambda=220 \mathrm{~nm}, 25^{\circ} \mathrm{C}$ ), tR (major) $=10.22 \mathrm{~min}, \mathrm{tR}$ (minor) $=$ $9.72 \mathrm{~min} ;{ }^{1} \mathbf{H}$ NMR $\left(400 \mathrm{MHz}, \mathrm{CDCl}_{3}\right) \delta=7.40-7.30(\mathrm{~m}, 4 \mathrm{H}), 7.30-7.24(\mathrm{~m}, 1 \mathrm{H}), 6.71(\mathrm{dd}, J=$ $15.8,1.4 \mathrm{~Hz}, 1 \mathrm{H}), 6.03(\mathrm{dd}, J=15.8,6.5 \mathrm{~Hz}, 1 \mathrm{H}), 3.54-3.42(\mathrm{~m}, 1 \mathrm{H}), 1.85-1.64(\mathrm{~m}, 6 \mathrm{H}), 1.58-$ $1.50(\mathrm{~m}, 2 \mathrm{H}), 1.35-1.11(\mathrm{~m}, 3 \mathrm{H}), 1.02-0.88(\mathrm{~m}, 2 \mathrm{H}) ;{ }^{13} \mathbf{C ~ N M R}\left(100 \mathrm{MHz}, \mathrm{CDCl}_{3}\right) \delta=135.8,132.9$, $128.7,128.2,126.5,123.6,120.4,40.9,35.1,33.2,32.5,31.8,26.3,25.9,25.9$. HRMS (APCI) for $\mathrm{C}_{17} \mathrm{H}_{21} \mathrm{~N}[\mathrm{M}]^{+}$: calcd 239.1669, found 239.1678.

\section{( $S, E)$-2-(Cyclopentylmethyl)-4-phenylbut-3-enenitrile (4ab)}

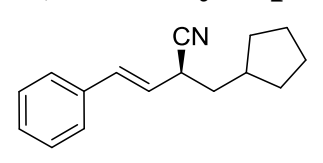

$73 \%(32.5 \mathrm{mg})$ isolated yield, light yellow oil, $[\alpha]_{\mathrm{D}}^{25}=1.80\left(\mathrm{c}=1.08\right.$ in $\left.\mathrm{CHCl}_{3}\right)$; 93\% ee, determined by HPLC analysis (Chiralpak AS-H column, hexane/i-PrOH, $95: 5 \mathrm{v} / \mathrm{v}$, flow rate $0.5 \mathrm{~mL} / \mathrm{min}, \lambda=250 \mathrm{~nm}, 25^{\circ} \mathrm{C}$ ), tR (major) $=14.67 \mathrm{~min}, \mathrm{tR}$ $($ minor $)=13.54 \mathrm{~min} ;{ }^{1} \mathbf{H}$ NMR $\left(400 \mathrm{MHz}, \mathrm{CDCl}_{3}\right) \delta=7.40-7.27(\mathrm{~m}, 5 \mathrm{H}), 6.71(\mathrm{~d}, J=15.8 \mathrm{~Hz}, 1 \mathrm{H})$, $6.05(\mathrm{dd}, J=15.8,6.6 \mathrm{~Hz}, 1 \mathrm{H}), 3.42(\mathrm{q}, J=6.9 \mathrm{~Hz}, 1 \mathrm{H}), 2.05(\mathrm{~m}, 1 \mathrm{H}), 1.88(\mathrm{~m}, 3 \mathrm{H}), 1.76-1.58(\mathrm{~m}$, $4 \mathrm{H}), 1.23-1.07(\mathrm{~m}, 2 \mathrm{H}) ;{ }^{13} \mathbf{C}$ NMR $\left(100 \mathrm{MHz}, \mathrm{CDCl}_{3}\right) \delta=135.8,132.9,128.7,128.2,126.5,123.5$, 120.4, 39.6, 37.7, 33.8, 32.6, 32.2, 25.1, 25.0. HRMS (ESI) for $\mathrm{C}_{16} \mathrm{H}_{19} \mathrm{NNa}[\mathrm{M}+\mathrm{Na}]^{+}$: calcd 248.1410, found 248.1399 .

\section{( $S, E)$-2-(Cyclopent-3-en-1-ylmethyl)-4-phenylbut-3-enenitrile (4ac)}

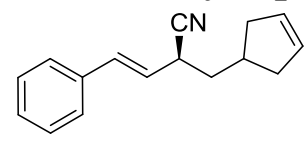

$69 \%(31.1 \mathrm{mg})$ isolated yield, light yellow oil, $[\alpha]_{\mathrm{D}}{ }^{25}=0.30\left(\mathrm{c}=1.10\right.$ in $\left.\mathrm{CHCl}_{3}\right)$; 92\% ee, determined by HPLC analysis (Chiralpak AS-H column, hexane $/ i$-PrOH, $95: 5 \mathrm{v} / \mathrm{v}$, flow rate $0.5 \mathrm{~mL} / \mathrm{min}, \lambda=250 \mathrm{~nm}, 25^{\circ} \mathrm{C}$ ), tR (major) $=15.10 \mathrm{~min}, \mathrm{tR}$ $($ minor $)=14.25 \mathrm{~min} ;{ }^{1} \mathbf{H}$ NMR $\left(400 \mathrm{MHz}, \mathrm{CDCl}_{3}\right) \delta=7.41-7.26(\mathrm{~m}, 5 \mathrm{H}), 6.72(\mathrm{~d}, J=15.8 \mathrm{~Hz}, 1 \mathrm{H})$, $6.05(\mathrm{dd}, J=15.8,6.6 \mathrm{~Hz}, 1 \mathrm{H}), 5.68(\mathrm{~s}, 2 \mathrm{H}), 2.61-2.49(\mathrm{~m}, 1 \mathrm{H}), 2.64-2.46(\mathrm{~m}, 3 \mathrm{H}), 2.05(\mathrm{dd}, J=$ 
11.8, 7.2 Hz, 2H), $2.00-1.76(\mathrm{~m}, 2 \mathrm{H}) ;{ }^{13} \mathbf{C ~ N M R}\left(100 \mathrm{MHz}, \mathrm{CDCl}_{3}\right) \delta=135.7,133.2,129.7,129.5$, $128.7,128.2,126.5,123.2,120.2,39.8,38.8,38.4,35.4,33.5$. HRMS (ESI) for $\mathrm{C}_{16} \mathrm{H}_{17} \mathrm{NNa}[\mathrm{M}+\mathrm{Na}]^{+}$: calcd 246.1253, found 246.1260 .

\section{$(S, E)$-2-Styrylhexanenitrile (4ad)}

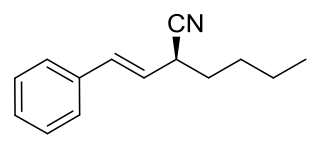

$73 \%(29.0 \mathrm{mg})$ isolated yield, light yellow oil, $[\alpha]_{\mathrm{D}}{ }^{25}=2.07\left(\mathrm{c}=0.96\right.$ in $\left.\mathrm{CHCl}_{3}\right)$;

96\% ee, determined by HPLC analysis (Chiralpak AS-H column, hexane $/ i-\mathrm{PrOH}$, 95:5 v/v, flow rate $0.5 \mathrm{~mL} / \mathrm{min}, \lambda=250 \mathrm{~nm}, 25^{\circ} \mathrm{C}$ ), tR (major) $=12.17 \mathrm{~min}, \mathrm{tR}$ $($ minor $)=11.46 \mathrm{~min} ;{ }^{1} \mathbf{H}$ NMR $\left(400 \mathrm{MHz}, \mathrm{CDCl}_{3}\right) \delta=7.44-7.21(\mathrm{~m}, 5 \mathrm{H}), 6.71(\mathrm{dd}, J=15.8,1.5 \mathrm{~Hz}$, $1 \mathrm{H}), 6.04(\mathrm{dd}, J=15.9,6.5 \mathrm{~Hz}, 1 \mathrm{H}), 3.41(\mathrm{q}, J=5.9 \mathrm{~Hz}, 1 \mathrm{H}), 1.87-1.71(\mathrm{~m}, 2 \mathrm{H}), 1.56-1.46(\mathrm{~m}$, $2 \mathrm{H}), 1.44-1.32(\mathrm{~m}, 2 \mathrm{H}), 0.93(\mathrm{t}, J=7.2 \mathrm{~Hz}, 3 \mathrm{H}) .{ }^{13} \mathbf{C} \mathbf{N M R}\left(100 \mathrm{MHz}, \mathrm{CDCl}_{3}\right) \delta=135.8,133.1$, 128.7, 128.2, 126.5, 123.3, 120.2, 34.3, 33.0, 28.9, 22.1, 13.8. HRMS (EI) for $\mathrm{C}_{14} \mathrm{H}_{17} \mathrm{NNa}[\mathrm{M}+\mathrm{Na}]^{+}$: calcd 222.1253, found 222.1244 .

\section{(S, E)-4-Methyl-2-styrylpentanenitrile (4ae)}

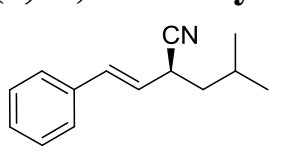

$69 \%(27.5 \mathrm{mg})$ isolated yield, light yellow oil, $[\alpha]_{\mathrm{D}}{ }^{25}=2.20\left(\mathrm{c}=0.92\right.$ in $\left.\mathrm{CHCl}_{3}\right)$;

$89 \%$ ee, determined by HPLC analysis (Chiralpak AS-H column, hexane $/ i$-PrOH, 95:5 v/v, flow rate $0.5 \mathrm{~mL} / \mathrm{min}, \lambda=250 \mathrm{~nm}, 25^{\circ} \mathrm{C}$ ), tR (major) $=12.63 \mathrm{~min}, \mathrm{tR}$ $($ minor $)=11.94 \mathrm{~min} ;{ }^{1} \mathbf{H}$ NMR $\left(400 \mathrm{MHz}, \mathrm{CDCl}_{3}\right) \delta=7.40-7.24(\mathrm{~m}, 5 \mathrm{H}), 6.72(\mathrm{dd}, J=15.8,1.4 \mathrm{~Hz}$, $1 \mathrm{H}), 6.03(\mathrm{dd}, J=15.8,6.6 \mathrm{~Hz}, 1 \mathrm{H}), 3.44(\mathrm{q}, J=6.5 \mathrm{~Hz}, 1 \mathrm{H}), 1.94-1.84(\mathrm{~m}, 1 \mathrm{H}), 1.78-1.71(\mathrm{~m}$, $1 \mathrm{H}), 1.58-1.51(\mathrm{~m}, 1 \mathrm{H}), 0.99(\mathrm{dd}, J=6.7,1.0 \mathrm{~Hz}, 6 \mathrm{H}) .{ }^{13} \mathbf{C}$ NMR $\left(100 \mathrm{MHz}, \mathrm{CDCl}_{3}\right) \delta=135.8$, 133.0, 128.7, 128.2, 126.5, 123.4, 120.3, 42.2, 32.5, 25.9, 22.5, 21.7. HRMS (ESI) for $\mathrm{C}_{14} \mathrm{H}_{17} \mathrm{NNa}$ $[\mathrm{M}+\mathrm{Na}]^{+}$: calcd 222.1253, found 222.1252.

\section{(S, E)-2-Ethyl-4-phenylbut-3-enenitrile (4af)}

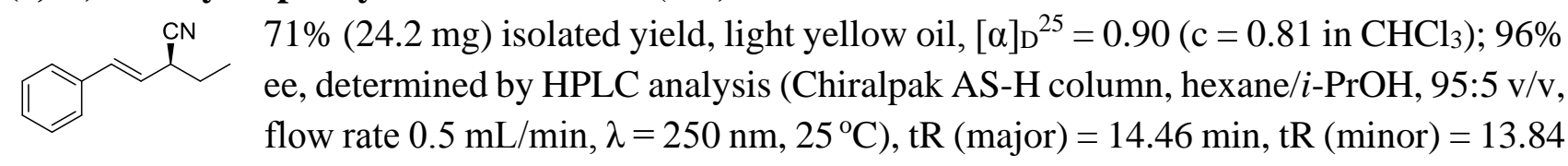
$\min ;{ }^{1} \mathbf{H}$ NMR $\left(400 \mathrm{MHz}, \mathrm{CDCl}_{3}\right) \delta=7.43-7.27(\mathrm{~m}, 5 \mathrm{H}), 6.73(\mathrm{~d}, J=15.8 \mathrm{~Hz}, 1 \mathrm{H}), 6.04(\mathrm{dd}, J=$ 15.8, $6.4 \mathrm{~Hz}, 1 \mathrm{H}), 3.39(\mathrm{q}, J=7.2 \mathrm{~Hz}, 1 \mathrm{H}), 1.91-1.73(\mathrm{~m}, 2 \mathrm{H}), 1.13(\mathrm{t}, J=7.4 \mathrm{~Hz}, 3 \mathrm{H}) .{ }^{\mathbf{1 3}} \mathbf{C} \mathbf{~ N M R}$ $\left(100 \mathrm{MHz} \mathrm{CDCl}_{3}\right) \delta=135.7,133.3,128.7,128.2,126.5,122.9,120.0,35.9,26.7,11.2$. HRMS (APCI) for $\mathrm{C}_{12} \mathrm{H}_{13} \mathrm{~N}[\mathrm{M}]^{+}$: calcd 171.1043, found 171.1047 .

\section{(S, E)-2-Styrylhept-6-enenitrile (4ag)}

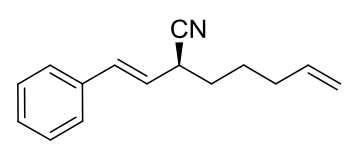

$74 \%$ (31.2 mg) isolated yield, light yellow oil, $[\alpha]_{\mathrm{D}}{ }^{25}=8.17\left(\mathrm{c}=1.04\right.$ in $\left.\mathrm{CHCl}_{3}\right)$;

$96 \%$ ee, determined by HPLC analysis (Chiralpak AZ-H column, hexane/i$\mathrm{PrOH}, 95: 5 \mathrm{v} / \mathrm{v}$, flow rate $0.5 \mathrm{~mL} / \mathrm{min}, \lambda=250 \mathrm{~nm}, 25^{\circ} \mathrm{C}$ ), tR (major) $=24.75$ $\min , \mathrm{tR}($ minor $)=23.94 \mathrm{~min} ;{ }^{1} \mathbf{H}$ NMR $\left(400 \mathrm{MHz}, \mathrm{CDCl}_{3}\right) \delta=7.41-7.26(\mathrm{~m}, 5 \mathrm{H}), 6.72(\mathrm{~d}, J=15.8$ $\mathrm{Hz}, 1 \mathrm{H}), 6.03(\mathrm{dd}, J=15.9,6.4 \mathrm{~Hz}, 1 \mathrm{H}), 5.86-5.71(\mathrm{~m}, 1 \mathrm{H}), 5.09-4.95(\mathrm{~m}, 2 \mathrm{H}), 3.43(\mathrm{q}, J=6.9 \mathrm{~Hz}$, $1 \mathrm{H}), 2.12(\mathrm{q}, J=7.2 \mathrm{~Hz}, 2 \mathrm{H}), 1.84-1.73(\mathrm{~m}, 2 \mathrm{H}), 1.70-1.57(\mathrm{~m}, 2 \mathrm{H}) .{ }^{\mathbf{1 3}} \mathbf{C} \mathbf{N M R}\left(100 \mathrm{MHz}, \mathrm{CDCl}_{3}\right)$ $\delta=137.5,135.7,133.2,128.7,128.2,126.5,123.0,120.1,115.4,34.3,33.0,32.6,25.9$. HRMS (ESI) for $\mathrm{C}_{15} \mathrm{H}_{17} \mathrm{NNa}[\mathrm{M}+\mathrm{Na}]^{+}$: calcd 234.1253.1253, found 234.1234. 


\section{(S, E)-2-Tyrylnon-8-ynenitrile (4ah)}

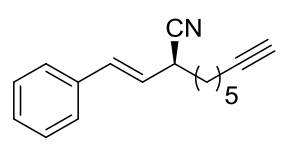

$79 \%(37.1 \mathrm{mg})$ isolated yield, light yellow oil, $[\alpha]_{\mathrm{D}}{ }^{25}=-1.50\left(\mathrm{c}=1.22\right.$ in $\left.\mathrm{CHCl}_{3}\right)$; 95\% ee, determined by HPLC analysis (Chiralpak AS-H column, hexane/ $i-\mathrm{PrOH}$, 95:5 v/v, flow rate $0.5 \mathrm{~mL} / \mathrm{min}, \lambda=250 \mathrm{~nm}, 25^{\circ} \mathrm{C}$ ), tR (major) $=21.03 \mathrm{~min}, \mathrm{tR}$ $($ minor $)=19.87 \mathrm{~min} ;{ }^{1} \mathbf{H}$ NMR $\left(400 \mathrm{MHz}, \mathrm{CDCl}_{3}\right) \delta=7.43-7.27(\mathrm{~m}, 5 \mathrm{H}), 6.72(\mathrm{~d}, J=15.8 \mathrm{~Hz}, 1 \mathrm{H})$, $6.03(\mathrm{dd}, J=15.8,6.4 \mathrm{~Hz}, 1 \mathrm{H}), 3.43(\mathrm{q}, J=6.9 \mathrm{~Hz}, 1 \mathrm{H}), 2.23-2.19(\mathrm{~m}, 2 \mathrm{H}), 1.95(\mathrm{~s}, 1 \mathrm{H}), 1.79$ (q, $J$ $=7.3 \mathrm{~Hz}, 2 \mathrm{H}), 1.62-1.44(\mathrm{~m}, 6 \mathrm{H}) .{ }^{13} \mathbf{C}$ NMR $\left(100 \mathrm{MHz}, \mathrm{CDCl}_{3}\right) \delta=135.7,133.2,128.7,128.2,126.5$, 123.0, 120.1, 84.2, 68.5, 34.3, 33.1, 28.0, 26.3, 18.2. HRMS (APCI) for $\mathrm{C}_{17} \mathrm{H}_{19} \mathrm{~N}[\mathrm{M}]^{+}$: calcd 237.1512, found 237.1523 .

\section{$(S, E)$-6-Chloro-2-styrylhexanenitrile (4ai)}

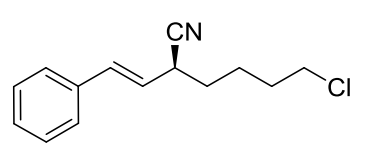

$71 \%(33.0 \mathrm{mg})$ isolated yield, light yellow oil, $[\alpha]_{\mathrm{D}}^{25}=6.83\left(\mathrm{c}=1.1\right.$ in $\left.\mathrm{CHCl}_{3}\right)$; $96 \%$ ee, determined by HPLC analysis (Chiralpak IE-H column, hexane/ $i$ -

$\mathrm{PrOH}, 95: 5 \mathrm{v} / \mathrm{v}$, flow rate $1.0 \mathrm{~mL} / \mathrm{min}, \lambda=254 \mathrm{~nm}, 25^{\circ} \mathrm{C}$ ), $\mathrm{tR}$ (major) $=12.18$ $\min , \mathrm{tR}($ minor $)=11.53 \mathrm{~min} ;{ }^{1} \mathbf{H}$ NMR $\left(400 \mathrm{MHz}, \mathrm{CDCl}_{3}\right) \delta=7.42-7.23(\mathrm{~m}, 5 \mathrm{H}), 6.73(\mathrm{~d}, J=15.8$ $\mathrm{Hz}, 1 \mathrm{H}), 6.03(\mathrm{~m}, 1 \mathrm{H}), 3.57-3.53(\mathrm{~m}, 2 \mathrm{H}), 3.44(\mathrm{q}, J=6.8 \mathrm{~Hz}, 1 \mathrm{H}), 1.89-1.76(\mathrm{~m}, 4 \mathrm{H}), 1.74-1.61$ $(\mathrm{m}, 2 \mathrm{H}) .{ }^{13} \mathbf{C ~ N M R}\left(100 \mathrm{MHz}, \mathrm{CDCl}_{3}\right) \delta=135.5,133.5,128.7,128.3,126.5,122.7,119.9,44.3,34.3$, 32.5, 31.8, 24.1. HRMS (ESI) for $\mathrm{C}_{14} \mathrm{H}_{16} \mathrm{ClNNa}[\mathrm{M}+\mathrm{Na}]^{+}$: calcd 256.0863, found 256.0867.

\section{(S, E)-2-(4-Methylstyryl)hexanenitrile (4bd)}

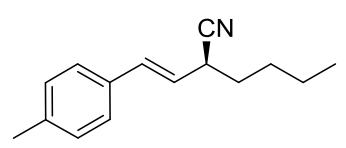

$67 \%(29.0 \mathrm{mg})$ isolated yield, light yellow oil, $[\alpha]_{\mathrm{D}}{ }^{25}=0.80\left(\mathrm{c}=0.97 \mathrm{in} \mathrm{CHCl}_{3}\right)$;

95\% ee, determined by HPLC analysis (Chiralpak IE-H column, hexane/i-PrOH, 95:5 v/v, flow rate $1.0 \mathrm{~mL} / \mathrm{min}, \lambda=254 \mathrm{~nm}, 25^{\circ} \mathrm{C}$ ), tR (major) $=8.97 \mathrm{~min}, \mathrm{tR}$ (minor) $=8.64 \mathrm{~min} ;{ }^{1} \mathbf{H}$ NMR $\left(400 \mathrm{MHz}, \mathrm{CDCl}_{3}\right) \delta=7.27(\mathrm{~d}, J=7.9 \mathrm{~Hz}, 2 \mathrm{H}), 7.14(\mathrm{~d}, J=7.8 \mathrm{~Hz}$, $2 \mathrm{H}), 6.67(\mathrm{~d}, J=15.7 \mathrm{~Hz}, 1 \mathrm{H}), 5.98(\mathrm{dd}, J=15.8,6.5 \mathrm{~Hz}, 1 \mathrm{H}), 3.40(\mathrm{q}, J=6.6 \mathrm{~Hz}, 1 \mathrm{H}), 2.34(\mathrm{~s}, 3 \mathrm{H})$, $1.74(\mathrm{~d}, J=7.3 \mathrm{~Hz}, 2 \mathrm{H}), 1.58-1.30(\mathrm{~m}, 4 \mathrm{H}), 0.93(\mathrm{t}, J=7.2 \mathrm{~Hz}, 3 \mathrm{H}) .{ }^{13} \mathbf{C} \mathbf{~ N M R}\left(100 \mathrm{MHz}, \mathrm{CDCl}_{3}\right)$ $\delta=138.1,132.9,129.4,126.4,122.1,120.3,34.3,33.0,28.9,22.1,21.2,13.8$. HRMS (APCI) for $\mathrm{C}_{15} \mathrm{H}_{19} \mathrm{~N}[\mathrm{M}]^{+}$: calcd 213.1512, found 213.1518.

\section{(S, E)-2-(4-Bromostyryl)hexanenitrile (4cd)}

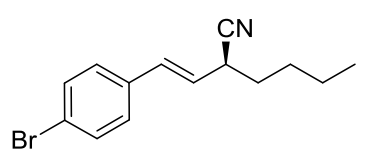

$56 \%(31.2 \mathrm{mg})$ isolated yield, light yellow oil, $[\alpha]_{\mathrm{D}}{ }^{25}=4.37(\mathrm{c}=1.04 \mathrm{in}$ $\mathrm{CHCl}_{3}$ ); 94\% ee, determined by HPLC analysis (Chiralpak AS-H column, hexane/i-PrOH, $95: 5 \mathrm{v} / \mathrm{v}$, flow rate $0.5 \mathrm{~mL} / \mathrm{min}, \lambda=250 \mathrm{~nm}, 25^{\circ} \mathrm{C}$ ), tR (major) $=16.24 \mathrm{~min}, \mathrm{tR}($ minor $)=18.52 \mathrm{~min} ;{ }^{1} \mathbf{H} \mathbf{~ N M R}\left(400 \mathrm{MHz}, \mathrm{CDCl}_{3}\right) \delta=7.46(\mathrm{~d}, J=8.5 \mathrm{~Hz}, 2 \mathrm{H}), 7.24$ $(\mathrm{d}, J=8.3 \mathrm{~Hz}, 2 \mathrm{H}), 6.66(\mathrm{~d}, J=15.8 \mathrm{~Hz}, 1 \mathrm{H}), 6.04(\mathrm{dd}, J=15.8,6.4 \mathrm{~Hz}, 1 \mathrm{H}), 3.41(\mathrm{q}, J=6.8 \mathrm{~Hz}, 1 \mathrm{H})$, 1.77 (q, J = 7.5 Hz, 2H), $1.55-1.31(\mathrm{~m}, 4 \mathrm{H}), 0.94(\mathrm{t}, J=7.2 \mathrm{~Hz}, 3 \mathrm{H}) .{ }^{13} \mathbf{C} \mathbf{N M R}\left(100 \mathrm{MHz}, \mathrm{CDCl}_{3}\right)$ $\delta=134.6,131.9,131.8,128.0,124.0,122.0,120.0,34.3,32.9,28.9,22.1,13.8$. HRMS (APCI) for $\mathrm{C}_{14} \mathrm{H}_{16} \mathrm{BrN}[\mathrm{M}]^{+}$: calcd 277.0461, found 277.0472.

\section{(S, E)-2-(4-Fluorostyryl)hexanenitrile (4dd)}

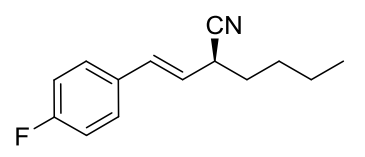

$63 \%(27.4 \mathrm{mg})$ isolated yield, light yellow oil, $[\alpha]_{\mathrm{D}}^{25}=1.50\left(\mathrm{c}=0.91 \mathrm{in} \mathrm{CHCl}_{3}\right)$; 97\% ee, determined by HPLC analysis (Chiralpak AS-H column, hexane/ $i$ $\mathrm{PrOH}, 99: 1 \mathrm{v} / \mathrm{v}$, flow rate $0.5 \mathrm{~mL} / \mathrm{min}, \lambda=254 \mathrm{~nm}, 25^{\circ} \mathrm{C}$ ), $\mathrm{tR}$ (major) $=18.32$ 
$\min , \mathrm{tR}($ minor $)=17.49 \mathrm{~min} ;{ }^{1} \mathbf{H}$ NMR $\left(400 \mathrm{MHz}, \mathrm{CDCl}_{3}\right) \delta=7.35(\mathrm{dd}, J=8.4,5.4 \mathrm{~Hz}, 2 \mathrm{H}), 7.03(\mathrm{t}$, $J=8.6 \mathrm{~Hz}, 2 \mathrm{H}), 6.68(\mathrm{~d}, J=15.8 \mathrm{~Hz}, 1 \mathrm{H}), 5.96(\mathrm{dd}, J=15.8,6.4 \mathrm{~Hz}, 1 \mathrm{H}), 3.41(\mathrm{q}, J=6.9 \mathrm{~Hz}, 1 \mathrm{H})$, $1.77(\mathrm{q}, J=7.6 \mathrm{~Hz}, 2 \mathrm{H}), 1.57-1.33(\mathrm{~m}, 4 \mathrm{H}), 0.94(\mathrm{t}, J=7.2 \mathrm{~Hz}, 3 \mathrm{H}) .{ }^{13} \mathbf{C ~ N M R}\left(100 \mathrm{MHz}, \mathrm{CDCl}_{3}\right)$ $\delta=163.9,161.4,132.0,128.2(\mathrm{~d}, J=8.0 \mathrm{~Hz}), 128.1,123.0,123.0,120.2,115.7(\mathrm{~d}, J=21.0 \mathrm{~Hz}), 34.4$, 33.0, 28.9, 22.2, 13.9. ${ }^{19} \mathbf{F}$ NMR $\left(376 \mathrm{MHz}, \mathrm{CDCl}_{3}\right) \delta=-113.39$. HRMS (APCI) for $\mathrm{C}_{14} \mathrm{H}_{16} \mathrm{FN}[\mathrm{M}]^{+}$: calcd 217.1261, found 217.1281.

\section{(S, E)-2-(4-(Trifluoromethyl)styryl)hexanenitrile (4ed)}

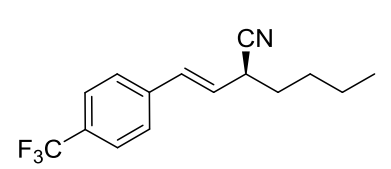

$62 \%(33.3 \mathrm{mg})$ isolated yield, light yellow oil, $[\alpha]_{\mathrm{D}}{ }^{25}=3.63(\mathrm{c}=1.10 \mathrm{in}$ $\mathrm{CHCl}_{3}$ ); $94 \%$ ee, determined by HPLC analysis (Chiralpak AS-H column, hexane $/ i$-PrOH, $95: 5 \mathrm{v} / \mathrm{v}$, flow rate $0.5 \mathrm{~mL} / \mathrm{min}, \lambda=250 \mathrm{~nm}, 25^{\circ} \mathrm{C}$ ), tR (major) $=12.79 \mathrm{~min}, \mathrm{tR}$ (minor) $=14.12 \mathrm{~min} ;{ }^{1} \mathbf{H} \mathbf{N M R}\left(400 \mathrm{MHz}, \mathrm{CDCl}_{3}\right) \delta=7.64-$ $7.44(\mathrm{~m}, 4 \mathrm{H}), 6.77(\mathrm{~d}, J=15.8 \mathrm{~Hz}, 1 \mathrm{H}), 6.15(\mathrm{dd}, J=15.9,6.2 \mathrm{~Hz}, 1 \mathrm{H}), 3.46(\mathrm{q}, J=6.7 \mathrm{~Hz}, 1 \mathrm{H}), 1.80$ $(\mathrm{q}, J=7.6 \mathrm{~Hz}, 2 \mathrm{H}), 1.55-1.34(\mathrm{~m}, 4 \mathrm{H}), 0.94(\mathrm{t}, J=7.2 \mathrm{~Hz}, 3 \mathrm{H}) .{ }^{13} \mathbf{C} \mathbf{N M R}\left(100 \mathrm{MHz}, \mathrm{CDCl}_{3}\right) \delta=$ $139.1,131.8,130.0(\mathrm{q}, J=33.0 \mathrm{~Hz}), 126.7,125.9,125.7$ (q, $J=4.0 \mathrm{~Hz}), 124.0(\mathrm{~d}, J=270.0 \mathrm{~Hz}), 119.8$, $34.4,32.8,28.9,22.1,13.8 .{ }^{19} \mathrm{~F} \mathrm{NMR}\left(376 \mathrm{MHz} \mathrm{CDCl}_{3}\right) \delta=-62.60$. HRMS (EI) for $\mathrm{C}_{15} \mathrm{H}_{16} \mathrm{~F}_{3} \mathrm{~N}[\mathrm{M}]^{+}$: calcd 267.1229, found 267.1239.

\section{(S, E)-2-(2-Methoxystyryl)hexanenitrile (4fd)}

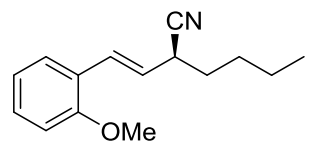

$58 \%(27.0 \mathrm{mg})$ isolated yield, light yellow oil, $[\alpha]_{\mathrm{D}}{ }^{25}=13.17\left(\mathrm{c}=0.90\right.$ in $\left.\mathrm{CHCl}_{3}\right)$; 96\% ee, determined by HPLC analysis (Chiralpak AS-H column, hexane/i-PrOH, 95: $5 \mathrm{v} / \mathrm{v}$, flow rate $0.5 \mathrm{~mL} / \mathrm{min}, \lambda=250 \mathrm{~nm}, 25^{\circ} \mathrm{C}$ ), tR (major) $=15.60 \mathrm{~min}, \mathrm{tR}$ (minor) $=14.95 \mathrm{~min} ;{ }^{1} \mathbf{H}$ NMR $\left(400 \mathrm{MHz}, \mathrm{CDCl}_{3}\right) \delta=7.39(\mathrm{dd}, J=7.6,1.7 \mathrm{~Hz}, 1 \mathrm{H}), 7.30-7.22(\mathrm{~m}$, $1 \mathrm{H}), 7.03-6.85(\mathrm{~m}, 3 \mathrm{H}), 6.09(\mathrm{dd}, J=15.9,6.8 \mathrm{~Hz}, 1 \mathrm{H}), 3.85(\mathrm{~s}, 3 \mathrm{H}), 3.41(\mathrm{q}, J=7.1,1 \mathrm{H}), 1.82-$ $1.72(\mathrm{~m}, 2 \mathrm{H}), 1.59-1.30(\mathrm{~m}, 4 \mathrm{H}), 0.93(\mathrm{t}, J=7.3 \mathrm{~Hz}, 3 \mathrm{H}) .{ }^{13} \mathbf{C} \mathbf{N M R}\left(100 \mathrm{MHz}, \mathrm{CDCl}_{3}\right) \delta=156.9$, $129.3,128.5,127.2$, 124.8, 124.0, 120.7, 120.5, 111.0, 55.4, 34.8, 33.2, 29.0, 22.2 13.8. HRMS (ESI) for $\mathrm{C}_{15} \mathrm{H}_{19} \mathrm{NNaO}[\mathrm{M}+\mathrm{Na}]^{+}$: calcd 252.1359, found 252.1351 .

\section{(S, E)-2-(3-Methylstyryl)hexanenitrile (4gd)}

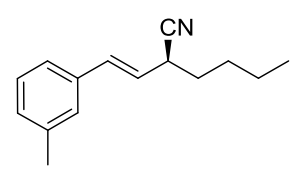

$61 \%(26.0 \mathrm{mg})$ isolated yield, light yellow oil, $[\alpha]_{\mathrm{D}}{ }^{25}=3.57\left(\mathrm{c}=0.87\right.$ in $\left.\mathrm{CHCl}_{3}\right)$;

94\% ee, determined by HPLC analysis (Chiralpak AS-H column, hexane $/ i$-PrOH, $95: 5 \mathrm{v} / \mathrm{v}$, flow rate $0.5 \mathrm{~mL} / \mathrm{min}, \lambda=250 \mathrm{~nm}, 25^{\circ} \mathrm{C}$ ), tR (major) $=12.01 \mathrm{~min}$, tR $($ minor $)=10.25 \mathrm{~min} ;{ }^{1} \mathbf{H}$ NMR $\left(400 \mathrm{MHz}, \mathrm{CDCl}_{3}\right) \delta=7.27-7.22(\mathrm{~m}, 1 \mathrm{H}), 7.21-$ $7.16(\mathrm{~m}, 2 \mathrm{H}), 7.10(\mathrm{~d}, J=7.4 \mathrm{~Hz}, 1 \mathrm{H}), 6.68(\mathrm{~d}, J=15.8 \mathrm{~Hz}, 1 \mathrm{H}), 6.03(\mathrm{dd}, J=15.8,6.5 \mathrm{~Hz}, 1 \mathrm{H}), 3.41$ $(\mathrm{q}, J=7.1 \mathrm{~Hz}, 1 \mathrm{H}), 2.35(\mathrm{~s}, 3 \mathrm{H}), 1.82-1.72(\mathrm{~m}, 2 \mathrm{H}), 1.55-1.33(\mathrm{~m}, 4 \mathrm{H}), 0.93(\mathrm{t}, J=7.2 \mathrm{~Hz}, 3 \mathrm{H})$. ${ }^{13} \mathrm{C} \mathrm{NMR}\left(100 \mathrm{MHz}, \mathrm{CDCl}_{3}\right) \delta=138.3,135.7,133.3,129.0,128.6,127.2,123.7,123.1,120.25,34.4$, 33.1, 28.9, 22.2, 21.4, 13.8. HRMS (ESI) for $\mathrm{C}_{15} \mathrm{H}_{19} \mathrm{NNa}[\mathrm{M}+\mathrm{Na}]^{+}$: calcd 234.1410, found 234.1407.

\section{(S, E)-2-(3-Fluorostyryl)hexanenitrile (4hd)}

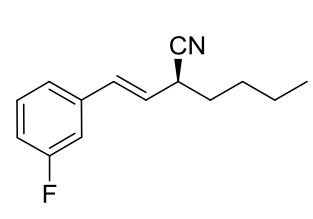

$50 \%(21.6 \mathrm{mg})$ isolated yield, light yellow oil, $[\alpha]_{\mathrm{D}}{ }^{25}=3.80\left(\mathrm{c}=0.72\right.$ in $\left.\mathrm{CHCl}_{3}\right)$; $92 \%$ ee, determined by HPLC analysis (Chiralpak AS-H column, hexane $/ i-\mathrm{PrOH}$, 95:5 v/v, flow rate $0.5 \mathrm{~mL} / \mathrm{min}, \lambda=250 \mathrm{~nm}, 25^{\circ} \mathrm{C}$ ), tR (major) $=13.91 \mathrm{~min}$, tR (minor) $=12.97 \mathrm{~min} ;{ }^{1} \mathbf{H}$ NMR $\left(400 \mathrm{MHz}, \mathrm{CDCl}_{3}\right) \delta=7.35-7.24(\mathrm{~m}, 1 \mathrm{H}), 7.11$ 
$(\mathrm{dd}, J=23.2,8.7 \mathrm{~Hz}, 2 \mathrm{H}), 6.98(\mathrm{t}, J=8.5 \mathrm{~Hz}, 1 \mathrm{H}), 6.70(\mathrm{~d}, J=15.9 \mathrm{~Hz}, 1 \mathrm{H}), 6.06(\mathrm{dd}, J=15.8,6.2$ $\mathrm{Hz}, 1 \mathrm{H}), 3.43(\mathrm{q}, J=7.0 \mathrm{~Hz}, 1 \mathrm{H}), 1.84-1.72(\mathrm{~m}, 2 \mathrm{H}), 1.55-1.33(\mathrm{~m}, 4 \mathrm{H}), 0.94(\mathrm{t}, J=7.2 \mathrm{~Hz}, 3 \mathrm{H})$. ${ }^{13}$ C NMR $\left(100 \mathrm{MHz}, \mathrm{CDCl}_{3}\right) \delta=164.3,161.8,138.0(\mathrm{~d}, J=7.7 \mathrm{~Hz}), 132.0(\mathrm{~d}, J=2.7 \mathrm{~Hz}), 130.2(\mathrm{~d}, J$ $=8.4 \mathrm{~Hz}), 124.7,122.5(\mathrm{~d}, J=2.7 \mathrm{~Hz}), 119.9,115.0(\mathrm{~d}, J=21.4 \mathrm{~Hz}), 112.9(\mathrm{~d}, J=22.0 \mathrm{~Hz}), 34.3,32.9$, 28.9, 22.1, 13.8. ${ }^{19} \mathrm{~F}$ NMR (376 MHz, $\left.\mathrm{CDCl}_{3}\right) \delta=-113.09$. HRMS (APCI) for $\mathrm{C}_{14} \mathrm{H}_{16} \mathrm{FN}[\mathrm{M}]^{+}$: calcd 217.1261, found 217.1245.

\section{(S, E)-2-(2-(Thiophen-3-yl)vinyl)hexanenitrile (4id)}

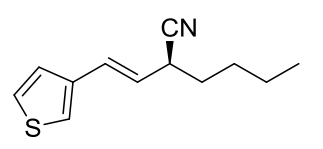

$70 \%(29.0 \mathrm{mg})$ isolated yield, light yellow oil, $[\alpha]_{\mathrm{D}}^{25}=7.53\left(\mathrm{c}=0.97\right.$ in $\left.\mathrm{CHCl}_{3}\right)$; 94\% ee, determined by HPLC analysis (Chiralpak AS-H column, hexane $/ i-\mathrm{PrOH}$, 95:5 v/v, flow rate $0.5 \mathrm{~mL} / \mathrm{min}, \lambda=250 \mathrm{~nm}, 25^{\circ} \mathrm{C}$ ), tR (major) $=18.46 \mathrm{~min}, \mathrm{tR}$ $($ minor $)=17.33 \mathrm{~min} ;{ }^{1} \mathbf{H}$ NMR $\left(400 \mathrm{MHz}, \mathrm{CDCl}_{3}\right) \delta=7.30(\mathrm{dd}, J=5.1,3.0 \mathrm{~Hz}, 1 \mathrm{H}), 7.24-7.16(\mathrm{~m}$, $2 \mathrm{H}), 6.72(\mathrm{~d}, J=15.8 \mathrm{~Hz}, 1 \mathrm{H}), 5.90(\mathrm{dd}, J=15.8,6.4 \mathrm{~Hz}, 1 \mathrm{H}), 3.38(\mathrm{q}, J=7.3,6.9 \mathrm{~Hz}, 1 \mathrm{H}), 1.75(\mathrm{q}$, $J=7.6 \mathrm{~Hz}, 2 \mathrm{H}), 1.57-1.32(\mathrm{~m}, 4 \mathrm{H}), 0.93(\mathrm{t}, J=7.2 \mathrm{~Hz}, 3 \mathrm{H}) .{ }^{13} \mathbf{C ~ N M R}\left(100 \mathrm{MHz}, \mathrm{CDCl}_{3}\right) \delta=138.2$, 127.2, 126.4, 124.7, 123.1, 123.0, 120.2, 34.2, 33.0, 28.8, 22.1, 13.8. HRMS (APCI) for $\mathrm{C}_{12} \mathrm{H}_{15} \mathrm{NS}$ $[\mathrm{M}]^{+}$: calcd 205.0920, found 205.0933.

\section{(S, E)-2-(2-(Furan-3-yl)vinyl)hexanenitrile (4jd)}

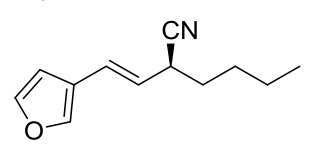

$73 \%(27.6 \mathrm{mg})$ isolated yield, light yellow oil, $[\alpha]_{\mathrm{D}}{ }^{25}=22.53\left(\mathrm{c}=0.92\right.$ in $\left.\mathrm{CHCl}_{3}\right)$; 95\% ee, determined by HPLC analysis (Chiralpak AD-H column, hexane $/ i-\mathrm{PrOH}$, 99:1 v/v, flow rate $0.5 \mathrm{~mL} / \mathrm{min}, \lambda=254 \mathrm{~nm}, 25^{\circ} \mathrm{C}$ ), tR (major) $=15.25 \mathrm{~min}, \mathrm{tR}$ $($ minor $)=14.85 \mathrm{~min} ;{ }^{1} \mathbf{H}$ NMR $\left(400 \mathrm{MHz}, \mathrm{CDCl}_{3}\right) \delta=7.50-7.36(\mathrm{~m}, 2 \mathrm{H}), 6.59(\mathrm{~d}, J=15.7 \mathrm{~Hz}, 1 \mathrm{H})$, $6.51(\mathrm{~d}, J=2.0 \mathrm{~Hz}, 1 \mathrm{H}), 5.77(\mathrm{dd}, J=15.7,6.4 \mathrm{~Hz}, 1 \mathrm{H}), 3.37(\mathrm{q}, J=7.4,6.9 \mathrm{~Hz}, 1 \mathrm{H}), 1.73(\mathrm{t}, J=7.5$ $\mathrm{Hz}, 2 \mathrm{H}), 1.58-1.30(\mathrm{~m}, 4 \mathrm{H}), 0.93(\mathrm{t}, J=7.2 \mathrm{~Hz}, 3 \mathrm{H}) .{ }^{13} \mathbf{C ~ N M R}\left(100 \mathrm{MHz}, \mathrm{CDCl}_{3}\right) \delta=143.8,141.0$, $122.9,122.8,122.7,120.2,107.2,77.3,77.0,76.7,34.3,33.0,28.8,22.1,13.8$. HRMS (ESI) for $\mathrm{C}_{12} \mathrm{H}_{15} \mathrm{NNaO}[\mathrm{M}+\mathrm{Na}]^{+}$: calcd 212.1046, found 212.1045 .

\section{(S, E)-2-(2-(Pyridin-3-yl)vinyl)hexanenitrile (4kd)}

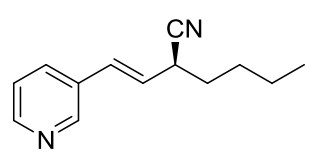

$45 \%(18.0 \mathrm{mg})$ isolated yield, light yellow oil, $[\alpha]_{\mathrm{D}}{ }^{25}=6.40\left(\mathrm{c}=0.60\right.$ in $\left.\mathrm{CHCl}_{3}\right)$; $70 \%$ ee, determined by HPLC analysis (Chiralpak OD-H column, hexane/i-PrOH, $90: 10 \mathrm{v} / \mathrm{v}$, flow rate $1.0 \mathrm{~mL} / \mathrm{min}, \lambda=254 \mathrm{~nm}, 25^{\circ} \mathrm{C}$ ), tR (major) $=25.52 \mathrm{~min}, \mathrm{tR}$ $($ minor $)=16.31 \mathrm{~min} ;{ }^{1} \mathbf{H}$ NMR $\left(400 \mathrm{MHz}, \mathrm{CDCl}_{3}\right) \delta=8.62(\mathrm{~s}, 1 \mathrm{H}), 8.52(\mathrm{~d}, J=4.8 \mathrm{~Hz}, 1 \mathrm{H}), 7.70(\mathrm{~d}$, $J=7.9 \mathrm{~Hz}, 1 \mathrm{H}), 7.32-7.25(\mathrm{~m}, 1 \mathrm{H}), 6.73(\mathrm{~d}, J=15.9 \mathrm{~Hz}, 1 \mathrm{H}), 6.14(\mathrm{dd}, J=15.9,6.2 \mathrm{~Hz}, 1 \mathrm{H}), 3.47$ $(\mathrm{q}, J=6.8 \mathrm{~Hz}, 1 \mathrm{H}), 1.62-1.33(\mathrm{~m}, 4 \mathrm{H}), 0.95(\mathrm{t}, J=7.2 \mathrm{~Hz}, 3 \mathrm{H}) .{ }^{13} \mathbf{C} \mathbf{N M R}\left(100 \mathrm{MHz}, \mathrm{CDCl}_{3}\right) \delta=$ 149.2, 148.2, 133.1, 131.4, 129.6, 125.6, 123.5, 119.8, 34.4, 32.8, 28.9, 22.1, 13.8. HRMS (ESI) for $\mathrm{C}_{13} \mathrm{H}_{17} \mathrm{~N}_{2}[\mathrm{M}+\mathrm{H}]^{+}$: calcd 201.1386, found 201.1386.

\section{(S, E)-2-(1-Phenylprop-1-en-2-yl)hexanenitrile (4ld)}

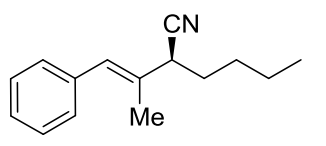

$70 \%(29.7 \mathrm{mg})$ isolated yield, light yellow oil, $[\alpha]_{\mathrm{D}}{ }^{25}=3.57\left(\mathrm{c}=0.99\right.$ in $\left.\mathrm{CHCl}_{3}\right)$; 97\% ee, determined by HPLC analysis (Chiralpak AS-H column, hexane/i-PrOH, 95:5 v/v, flow rate $0.5 \mathrm{~mL} / \mathrm{min}, \lambda=250 \mathrm{~nm}, 25^{\circ} \mathrm{C}$ ), tR (major) $=11.39 \mathrm{~min}, \mathrm{tR}$ $($ minor $)=10.18 \mathrm{~min} ;{ }^{1} \mathbf{H}$ NMR $\left(400 \mathrm{MHz}, \mathrm{CDCl}_{3}\right) \delta=7.39-7.31(\mathrm{~m}, 2 \mathrm{H}), 7.28-7.20(\mathrm{~m}, 3 \mathrm{H}), 6.56$ (s, 1H), $3.33-3.23(\mathrm{~m}, 1 \mathrm{H}), 1.94(\mathrm{~d}, J=1.5 \mathrm{~Hz}, 3 \mathrm{H}), 1.89-1.71(\mathrm{~m}, 2 \mathrm{H}), 1.55-1.33(\mathrm{~m}, 4 \mathrm{H}), 0.94$ 
$(\mathrm{t}, J=7.1 \mathrm{~Hz}, 3 \mathrm{H}) .{ }^{13} \mathrm{C}$ NMR $\left(100 \mathrm{MHz}, \mathrm{CDCl}_{3}\right) \delta=136.7,131.9,129.1,128.8,128.2,127.0,120.5$, 41.1, 31.4, 29.1, 22.1, 15.5, 13.8. HRMS (ESI) for $\mathrm{C}_{15} \mathrm{H}_{19} \mathrm{NNa}[\mathrm{M}+\mathrm{Na}]^{+}$: calcd 236.1410, found 236.1396.

\section{(R, Z)-2-(1-Bromo-2-phenylvinyl)hexanenitrile (4md)}

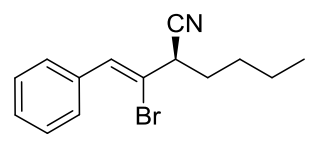

$42 \%(23.2 \mathrm{mg})$ isolated yield, light yellow oil, $[\alpha]_{\mathrm{D}}{ }^{25}=1.97\left(\mathrm{c}=0.77\right.$ in $\left.\mathrm{CHCl}_{3}\right)$; 97\% ee, determined by HPLC analysis (Chiralpak AS-H column, hexane $/ i-\mathrm{PrOH}$, $95: 5 \mathrm{v} / \mathrm{v}$, flow rate $0.5 \mathrm{~mL} / \mathrm{min}, \lambda=250 \mathrm{~nm}, 25^{\circ} \mathrm{C}$ ), tR (major) $=16.79 \mathrm{~min}$, tR $($ minor $)=12.77 \mathrm{~min} ;{ }^{1} \mathbf{H}$ NMR $\left(400 \mathrm{MHz}, \mathrm{CDCl}_{3}\right) \delta=7.62-7.54(\mathrm{~m}, 2 \mathrm{H}), 7.43-7.31(\mathrm{~m}, 3 \mathrm{H}), 7.11$ $(\mathrm{s}, 1 \mathrm{H}), 3.66(\mathrm{t}, J=7.1 \mathrm{~Hz}, 1 \mathrm{H}), 1.99(\mathrm{q}, J=7.5 \mathrm{~Hz}, 2 \mathrm{H}), 1.54-1.37(\mathrm{~m}, 4 \mathrm{H}), 0.95(\mathrm{t}, J=7.0 \mathrm{~Hz}, 3 \mathrm{H})$. ${ }^{13} \mathrm{C}$ NMR $\left(100 \mathrm{MHz}, \mathrm{CDCl}_{3}\right) \delta=134.5,131.5,129.0,128.6,128.3,119.2,118.8,43.5,32.0,28.4$, 22.0, 13.8. HRMS (ESI) for $\mathrm{C}_{14} \mathrm{H}_{16} \mathrm{BrNNa}[\mathrm{M}+\mathrm{Na}]^{+}$: calcd 300.0358, found 300.0360.

\section{(S, E)-2-(2-(4-Fluorophenyl)prop-1-en-1-yl)hexanenitrile (4nd)}

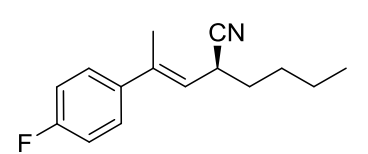

$60 \%(27.8 \mathrm{mg})$ isolated yield, light yellow oil, $[\alpha]_{\mathrm{D}}{ }^{25}=6.50\left(\mathrm{c}=0.92\right.$ in $\left.\mathrm{CHCl}_{3}\right)$; $74 \%$ ee, determined by HPLC analysis (Chiralpak AS-H column, hexane/ $i$ $\mathrm{PrOH}, 95: 5 \mathrm{v} / \mathrm{v}$, flow rate $0.5 \mathrm{~mL} / \mathrm{min}, \lambda=250 \mathrm{~nm}, 25^{\circ} \mathrm{C}$ ), tR (major) $=14.57$ $\min , \mathrm{tR}$ (minor) $=11.84 \mathrm{~min} ;{ }^{1} \mathbf{H}$ NMR $\left(400 \mathrm{MHz}, \mathrm{CDCl}_{3}\right) \delta=7.38-7.28(\mathrm{~m}, 2 \mathrm{H}), 7.02(\mathrm{t}, J=8.7$ $\mathrm{Hz}, 2 \mathrm{H}), 5.59-5.55(\mathrm{~m}, 1 \mathrm{H}), 3.52-3.46(\mathrm{~m}, 1 \mathrm{H}), 2.08(\mathrm{~d}, J=1.4 \mathrm{~Hz}, 3 \mathrm{H}), 1.88-1.79(\mathrm{~m}, 1 \mathrm{H}), 1.74$ - $1.66(\mathrm{~m}, 1 \mathrm{H}), 1.55-1.45(\mathrm{~m}, 2 \mathrm{H}), 1.44-1.33(\mathrm{~m}, 2 \mathrm{H}), 0.94(\mathrm{t}, J=7.2 \mathrm{~Hz}, 3 \mathrm{H}) .{ }^{13} \mathbf{C} \mathbf{~ N M R}(100$ $\left.\mathrm{MHz}_{1} \mathrm{CDCl}_{3}\right) \delta=163.7,161.2,139.0,138.2,127.5(\mathrm{~d}, J=8.0 \mathrm{~Hz}), 121.7,120.7,115.2(\mathrm{~d}, J=21.0$ $\mathrm{Hz}$ ), 33.0, 30.3, 29.0, 22.2, 16.6, 13.8. $\left.{ }^{19} \mathrm{~F} \mathrm{NMR} \mathrm{(376} \mathrm{MHz,} \mathrm{CDCl}_{3}\right) \delta=-114.61$. HRMS (APCI) for $\mathrm{C}_{15} \mathrm{H}_{18} \mathrm{FN}[\mathrm{M}]^{+}$: calcd 231.1418, found 231.1429.

\section{Mixture of (1S, 7R)- and (1S, 7R) -3-Phenyl-7-propylcyclohept-2-ene-1-carbonitrile (4od)}

$36 \%(17.2 \mathrm{mg})$ isolated yield, light yellow oil, $[\alpha]_{\mathrm{D}}^{25}=-2.57\left(\mathrm{c}=0.54\right.$ in $\left.\mathrm{CHCl}_{3}\right)$; trans $/ \mathrm{cis}$
$=2.8: 1,79 \%$ ee, $96 \%$ ee, determined by HPLC analysis (Chiralpak AS column, hexane $/ i-$ $\mathrm{PrOH}, 99: 1 \mathrm{v} / \mathrm{v}$, flow rate $0.5 \mathrm{~mL} / \mathrm{min}, \lambda=254 \mathrm{~nm}, 25^{\circ} \mathrm{C}$ ), tR (major) $=20.46 \mathrm{~min}$, tR $($ minor $)=13.28 \mathrm{~min} ; \mathrm{tR}($ major $)=19.30 \mathrm{~min}, \mathrm{tR}($ minor $)=12.59 \mathrm{~min} ;{ }^{1} \mathbf{H} \mathbf{N M R}\left(400 \mathrm{MHz}, \mathrm{CD}_{3} \mathrm{OD}\right)$ $\delta=7.36-7.22(\mathrm{~m}, 7 \mathrm{H}), 5.89(\mathrm{~d}, J=8.1 \mathrm{~Hz}, 1 \mathrm{H}), 5.82(\mathrm{~d}, J=7.4 \mathrm{~Hz}, 1 \mathrm{H}), 3.77(\mathrm{~d}, J=8.3 \mathrm{~Hz}, 1 \mathrm{H})$, $3.70(\mathrm{t}, J=7.2 \mathrm{~Hz}, 1 \mathrm{H}), 2.84-2.77(\mathrm{~m}, 1 \mathrm{H}), 2.69-2.62(\mathrm{~m}, 1 \mathrm{H}), 2.24-2.16(\mathrm{~m}, 1 \mathrm{H}), 2.06-1.69(\mathrm{~m}$, $5 \mathrm{H}), 1.70-1.51(\mathrm{~m}, 3 \mathrm{H}), 1.50-1.24(\mathrm{~m}, 7 \mathrm{H}), 0.94(\mathrm{t}, J=7.1 \mathrm{~Hz}, 4 \mathrm{H}) .{ }^{13} \mathbf{C} \mathbf{N M R}\left(150 \mathrm{MHz}, \mathrm{CDCl}_{3}\right)$ $\delta=149.3,148.6,143.3,143.1,128.3,127.5,125.9,121.2,121.0,120.8,119.7,38.8,37.6,36.0,35.2$, $35.1,34.9,34.28,33.7,32.0,24.3,22.4,20.3,19.9,14.1,14.0$. HRMS (APCI) for $\mathrm{C}_{12} \mathrm{H}_{21} \mathrm{~N}[\mathrm{M}]^{+}$: calcd 239.1669, found 239.1681.

\section{(S, E)-2-Butylundec-3-enenitrile (4qd)}

$\mathrm{CN}_{\mathrm{N}} \quad 33 \%(14.5 \mathrm{mg})$ isolated yield, light yellow oil, $[\alpha]_{\mathrm{D}}^{25}=3.73(\mathrm{c}=1.45 \mathrm{in}$ $\mathrm{CHCl}_{3}$ ); $7 \%$ ee, determined by HPLC analysis (Chiralpak AS-H column, hexane $/ i$-PrOH, $95: 5 \mathrm{v} / \mathrm{v}$, flow rate $0.5 \mathrm{~mL} / \mathrm{min}, \lambda=250 \mathrm{~nm}, 25^{\circ} \mathrm{C}$ ), tR (major) $=8.39 \mathrm{~min}$, tR (minor) $=8.76 \mathrm{~min} ;{ }^{1} \mathbf{H}$ NMR $\left(400 \mathrm{MHz}, \mathrm{CDCl}_{3}\right) \delta=5.82-5.75(\mathrm{~m}, 1 \mathrm{H}), 5.31(\mathrm{dd}, J=15.3,6.5 \mathrm{~Hz}, 1 \mathrm{H})$, $3.19(\mathrm{q}, J=7.0 \mathrm{~Hz}, 1 \mathrm{H}), 2.05(\mathrm{q}, J=7.6 \mathrm{~Hz}, 2 \mathrm{H}), 1.45-1.27(\mathrm{~m}, 13 \mathrm{H}), 0.94-0.87(\mathrm{~m}, 7 \mathrm{H}) .{ }^{13} \mathbf{C} \mathbf{N M R}$ $\left(100 \mathrm{MHz} \mathrm{CDCl}_{3}\right) \delta=134.8,123.6,120.8,34.0,33.2,33.0,32.1,31.8,31.6,31.5,31.0,28.8,28.7$, 
28.6, 26.7, 22.6, 22.5, 22.1, 14.1, 14.0, 13.9, 13.8. HRMS (APCI) for $\mathrm{C}_{15} \mathrm{H}_{27} \mathrm{~N}[\mathrm{M}]^{+}$: calcd 221.2138, found 221.2120 .

$(2 S, 6 R)-6-((5 S, 8 R, 9 S, 10 S, 13 R, 14 S, 17 R)-10,13-D i m e t h y l-3,7,12$ trioxohexadecahy-dro-1Hcyclopenta[a]phenanthren-17-yl)-2-((E)-styryl)heptanenitrile (4aj)

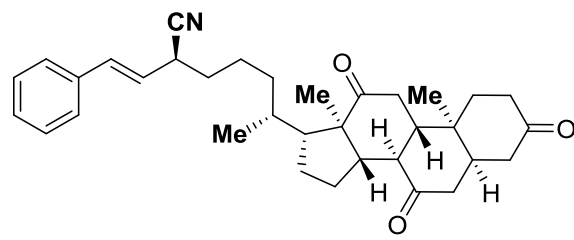

$41 \%(42.1 \mathrm{mg})$ isolated yield, white solid, $[\alpha]_{\mathrm{D}}{ }^{25}=27.73(\mathrm{c}=1.40$ in $\left.\mathrm{CHCl}_{3}\right)$; d.r. >19:1; ${ }^{1} \mathbf{H}$ NMR (600 $\left.\mathrm{MHz}, \mathrm{CDCl}_{3}\right) \delta=7.39-$ $7.33(\mathrm{~m}, 4 \mathrm{H}), 7.28(\mathrm{~d}, J=7.6 \mathrm{~Hz}, 1 \mathrm{H}), 6.72(\mathrm{~d}, J=15.6 \mathrm{~Hz}, 1 \mathrm{H})$, $6.04(\mathrm{dd}, J=15.9,6.4 \mathrm{~Hz}, 1 \mathrm{H}), 4.12(\mathrm{q}, J=7.2 \mathrm{~Hz}, 1 \mathrm{H}), 3.43(\mathrm{q}$, $\mathrm{J}=7.0 \mathrm{~Hz}, 1 \mathrm{H}), 2.95-2.82(\mathrm{~m}, 3 \mathrm{H}), 2.38-2.26(\mathrm{~m}, 4 \mathrm{H}), 2.23-$ $2.20(\mathrm{~m}, 2 \mathrm{H}), 2.18-2.09(\mathrm{~m}, 2 \mathrm{H}), 2.04-1.92(\mathrm{~m}, 4 \mathrm{H}), 1.91-1.71(\mathrm{~m}, 4 \mathrm{H}), 1.66-1.59(\mathrm{~m}, 2 \mathrm{H})$, $1.52-1.45(\mathrm{~m}, 1 \mathrm{H}), 1.40(\mathrm{~s}, 3 \mathrm{H}), 1.30-1.23(\mathrm{~m}, 5 \mathrm{H}), 1.17(\mathrm{~s}, 1 \mathrm{H}), 1.07(\mathrm{~s}, 3 \mathrm{H}), 0.85(\mathrm{~d}, \mathrm{~J}=6.6 \mathrm{~Hz}$, $3 \mathrm{H}) .{ }^{13} \mathrm{C}$ NMR $\left(100 \mathrm{MHz}, \mathrm{CDCl}_{3}\right) \delta=212.0,209.1,208.7,135.7,133.2,128.6,128.2,126.5,123.1$, 120.2, 56.8, 51.7, 48.9, 46.7, 45.7, 45.5, 44.9, 42.7, 38.6, 36.4, 36.0, 35.2, 34.7, 34.3, 33.6, 31.5, 27.8, 25.1, 23.9, 22.6, 21.8, 18.9, 14.1, 11.8. HRMS (ESI) for $\mathrm{C}_{34} \mathrm{H}_{43} \mathrm{NNaO}_{3}[\mathrm{M}+\mathrm{Na}]^{+}$: calcd 536.3135, found 536.3157 .

\section{Synthetic Application of the Reaction}

\subsection{Scaled-Up Reaction}

This reaction was conducted according to the general procedure $\mathrm{C}$ : In an argon-filled glove box, a flame-dried $100 \mathrm{ml}$ Schlenk tube equipped with a magnetic stirrer bar was charged sequentially with $\mathbf{C u}\left(\mathbf{C H}_{3} \mathbf{C N}\right)_{4} \mathbf{B F}_{4}(157.5 \mathrm{mg}, 0.5 \mathrm{mmol})$ and $\mathbf{L 1}(214.0 \mathrm{mg}, 0.6 \mathrm{mmol})$, followed by the addition of DCE $(25 \mathrm{~mL})$. The mixture was then stirred at room temperature for 30 minutes. $N$ (Acyloxy)phthalimides 2d (10 mmol), organo-photocatalyst Perylene (126.2 mg, $0.5 \mathrm{mmol}), 1,3$ diene $1 \mathbf{a}(30 \mathrm{mmol})$ and TMSCN $(30 \mathrm{mmol})$ were added to the resulting mixture. The vial was closed and the Schlenk tube was removed from the glove box. At last, the mixture was stirred at a distance of $\sim 1 \mathrm{~cm}$ from a $2 \times 3 \mathrm{~W}$ white LEDs at $29^{\circ} \mathrm{C}$ about $96 \mathrm{~h}$ until the reaction was completed, as monitored by TLC analysis. The product was purified by flash column chromatography on silica gel (petrol ether/ EtOAc $=100 / 1)$ to afford the desired product.
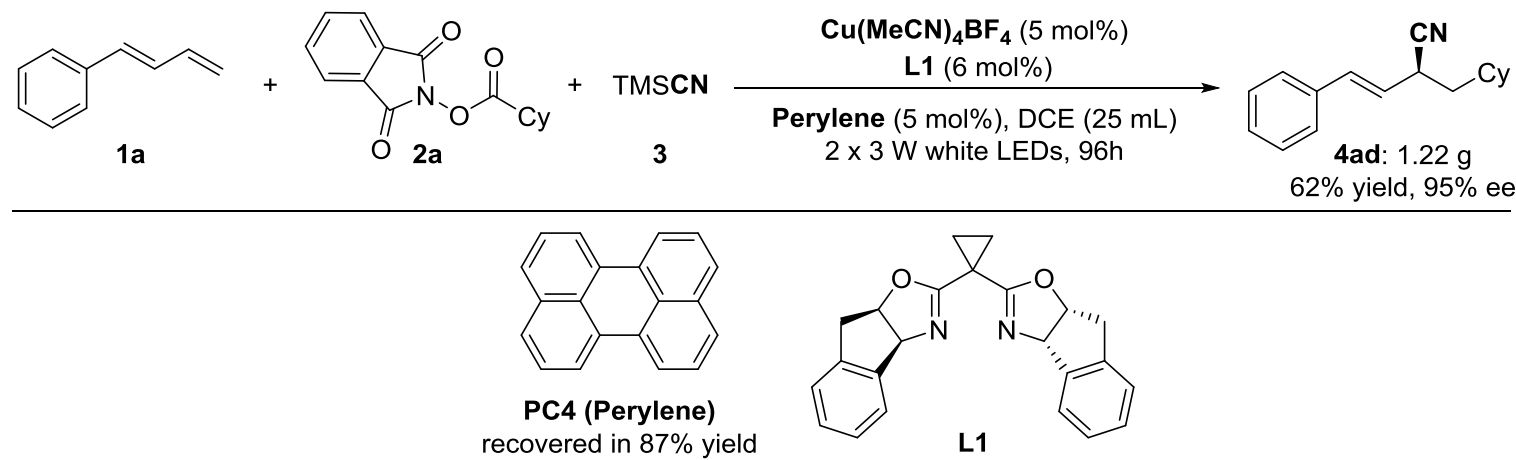

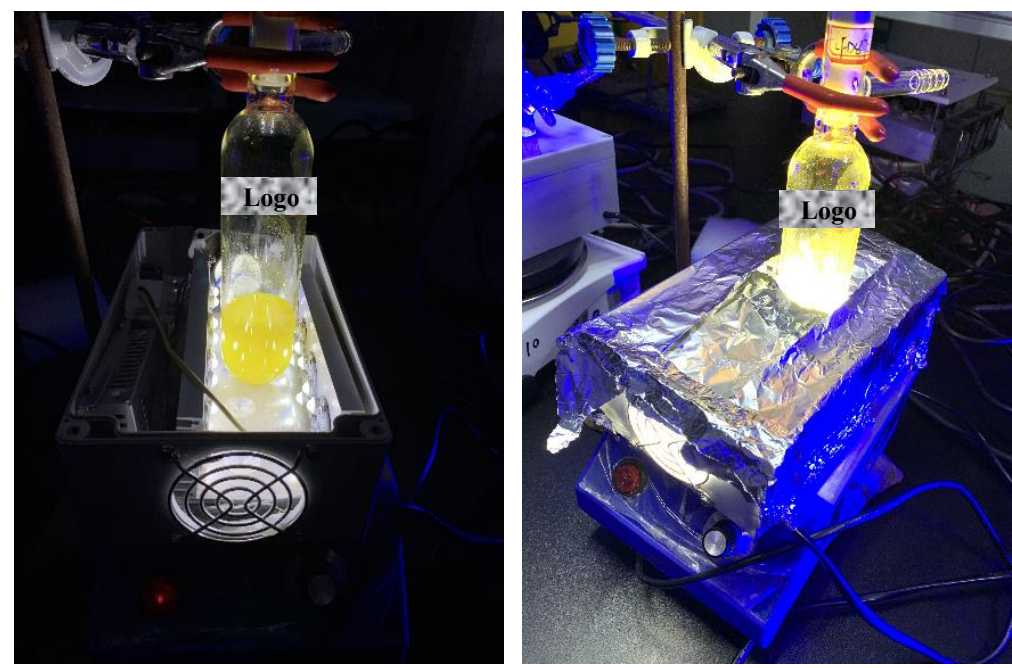

Figure S7. Scaled-up reaction

\subsection{Reduction of Double Bond}
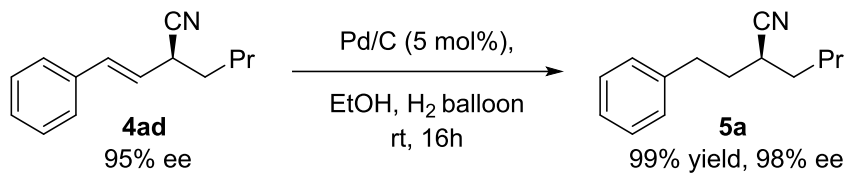

To a flame-dried Schlenk bottle, 4ad (20.1 mg, $0.1 \mathrm{mmol}, 1.0$ equiv, 95\% ee), $\mathrm{Pd} / \mathrm{C}$ (10 mg, 5 $\mathrm{wt} \%)$ were dissolved in EtOH $(40 \mathrm{~mL})$. The mixture was then bubbled with hydrogen gas for 20 minutes. Hydrogen balloon was inserted on the branch port of the Schlenk bottle and then the mixture stirred at room temperature for 16 hours. After that, the mixture was filtered through a pad of celite and washed with EtOAc quickly. The filtrate was concentrated under reduced pressure and then purified by flash column chromatography on silica gel to afford 5a (20.0 mg, 99\% yield, 98\% ee) as colorless oil. $[\alpha]_{\mathrm{D}}{ }^{25}=15.3\left(\mathrm{c}=0.66\right.$ in $\left.\mathrm{CHCl}_{3}\right)$; ee was determined by HPLC analysis (Chiralpak AD$\mathrm{H}$ column, hexane $/ i-\mathrm{PrOH}, 99: 1 \mathrm{v} / \mathrm{v}$, flow rate $0.5 \mathrm{~mL} / \mathrm{min}, \lambda=254 \mathrm{~nm}, 25^{\circ} \mathrm{C}$ ), tR (major) $=8.49 \mathrm{~min}$, $\mathrm{tR}$ (minor) $=8.76 \mathrm{~min} ;{ }^{1} \mathbf{H}$ NMR $\left(400 \mathrm{MHz}, \mathrm{CDCl}_{3}\right) \delta=7.32-7.28(\mathrm{~m}, 2 \mathrm{H}), 7.25-7.16(\mathrm{~m}, 3 \mathrm{H})$, 2.92-2.85 (m, 1H), 2.77-2.70 (m, 1H), 2.52-2.45 (m, 1H), $2.01-1.79(\mathrm{~m}, 2 \mathrm{H}), 1.70-1.46(\mathrm{~m}, 4 \mathrm{H})$, $1.42-1.29(\mathrm{~m}, 2 \mathrm{H}), 0.91(\mathrm{t}, J=7.2 \mathrm{~Hz}, 3 \mathrm{H}) .{ }^{13} \mathbf{C ~ N M R}\left(100 \mathrm{MHz}, \mathrm{CDCl}_{3}\right) \delta=140.3,128.6,128.4$, 126.3, 122.1, 34.0, 33.3, 31.9, 31.0, 29.2, 22.2, 13.8. HRMS (APCI) for $\mathrm{C}_{14} \mathrm{H}_{19} \mathrm{~N}[\mathrm{M}]^{+}$: calcd 201.1512, found 201.1517 .

\subsection{Reduction of Double Bond and Cyano Group}
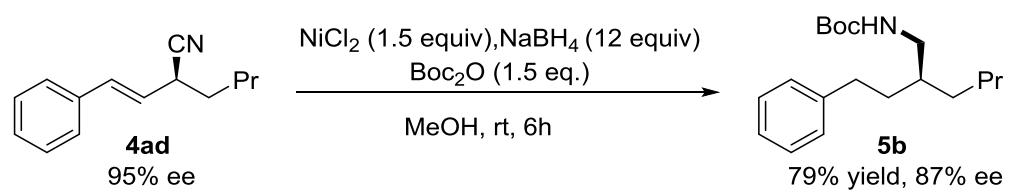

To a flame-dried $25 \mathrm{~mL}$ Schlenk bottle, 4 ad $\left(20.1 \mathrm{mg}, 0.1 \mathrm{mmol}, 1.0\right.$ equiv, $95 \%$ ee), $\mathrm{NiCl}_{2}(19.5$ $\mathrm{mg}, 0.15 \mathrm{mmol}, 1.5$ equiv) and $\mathrm{Boc}_{2} \mathrm{O}(65.4 \mathrm{mg}, 0.3 \mathrm{mmol}, 3.0$ equiv) were dissolved in $\mathrm{MeOH}$ (10 $\mathrm{mL}$ ). The mixture was cooled to $0{ }^{\circ} \mathrm{C}$ and $\mathrm{NaBH}_{4}(45.3 \mathrm{mg}, 1.2 \mathrm{mmol}, 12.0$ equiv) was then added slowly. The reaction system was stirred at r.t. for $6 \mathrm{~h}$. After completion of the reaction (TLC), the reaction was quenched with saturated $\mathrm{NH}_{4} \mathrm{Cl}$ solution and the mixture was extracted with EtOAc. The 
concentrated organic layer was dried over anhydrous $\mathrm{Na}_{2} \mathrm{SO}_{4}$. The organic layer was then concentrated under reduced pressure and purified by flash column chromatography on silica gel to afford $\mathbf{5 b}$ (24.0 $\mathrm{mg}, 79 \%$ yield, $87 \%$ ee) as colorless oil. $[\alpha] \mathrm{D}^{25}=-1.13\left(\mathrm{c}=0.80\right.$ in $\left.\mathrm{CHCl}_{3}\right)$; ee was determined by HPLC analysis (Chiralpak OJ-H column, hexane/i-PrOH, 95:5 v/v, flow rate $1.0 \mathrm{~mL} / \mathrm{min}, \lambda=220 \mathrm{~nm}$, $\left.25{ }^{\circ} \mathrm{C}\right)$, tR (major) $=10.38 \mathrm{~min}, \mathrm{tR}$ (minor) $=9.30 \mathrm{~min} ;{ }^{1} \mathbf{H} \mathbf{N M R}\left(400 \mathrm{MHz}, \mathrm{CDCl}_{3}\right) \delta=7.27(\mathrm{dd}, J=$ $8.2,6.8 \mathrm{~Hz}, 2 \mathrm{H}), 7.18(\mathrm{~d}, J=7.3 \mathrm{~Hz}, 3 \mathrm{H}), 4.50(\mathrm{~s}, 1 \mathrm{H}), 3.13(\mathrm{q}, J=6.1 \mathrm{~Hz}, 2 \mathrm{H}), 2.63(\mathrm{dd}, J=9.6,6.4$ $\mathrm{Hz}, 2 \mathrm{H}), 1.64-1.49(\mathrm{~m}, 3 \mathrm{H}), 1.47(\mathrm{~s}, 9 \mathrm{H}), 1.35-1.23(\mathrm{~m}, 6 \mathrm{H}), 0.90(\mathrm{q}, J=6.5,3 \mathrm{H}) .{ }^{13} \mathbf{C} \mathbf{N M R}(100$ $\left.\mathrm{MHz}, \mathrm{CDCl}_{3}\right) \delta=156.1,142.6,128.3,125.7,79.0,43.5,38.0,33.6,33.0,31.3,28.8,28.4,23.0,14.0$. HRMS (APCI) for $\mathrm{C} 19 \mathrm{H} 31 \mathrm{NO}_{2}[\mathrm{M}]+$ : calcd 305.2349, found 305.2345.

\subsection{Alcoholysis of Cyano Group}
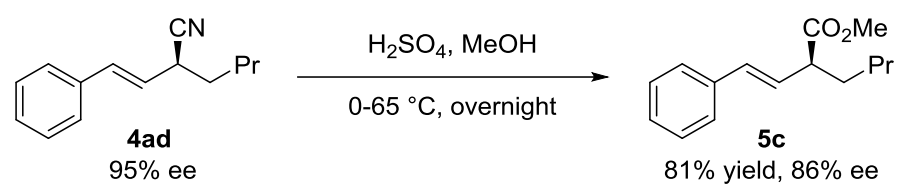

To a flame-dried $10 \mathrm{~mL}$ sealing tube, 4 ad $(20.1 \mathrm{mg}, 0.1 \mathrm{mmol}, 1.0$ equiv, $95 \%$ ee $)$ was dissolved in $\mathrm{MeOH}(0.8 \mathrm{~mL})$. The mixture was cooled to $0{ }^{\circ} \mathrm{C}$ and concentrated $\mathrm{H}_{2} \mathrm{SO}_{4}(0.35 \mathrm{~mL})$ was then added slowly. The reaction system was stirred at $65^{\circ} \mathrm{C}$ for overnight. After completion of the reaction (TLC), the reaction was quenched with cold water and the mixture was extracted with EtOAc. The concentrated organic layer was dried over anhydrous $\mathrm{Na}_{2} \mathrm{SO}_{4}$. The organic layer was then concentrated under reduced pressure and purified by flash column chromatography on silica gel to afford $\mathbf{5 c}$ (18.7 $\mathrm{mg}, 81 \%$ yield, $86 \%$ ee $)$ as colorless oil. $[\alpha]_{\mathrm{D}}{ }^{25}=8.23\left(\mathrm{c}=0.62\right.$ in $\left.\mathrm{CHCl}_{3}\right)$; ee was determined by HPLC analysis (Chiralpak IC-H column, hexane $/ i-\mathrm{PrOH}, 95: 5 \mathrm{v} / \mathrm{v}$, flow rate $1.0 \mathrm{~mL} / \mathrm{min}, \lambda=220 \mathrm{~nm}$, $\left.25^{\circ} \mathrm{C}\right), \mathrm{tR}$ (major) $=9.05 \mathrm{~min}, \mathrm{tR}$ (minor) $=9.47 \mathrm{~min} ;{ }^{1} \mathbf{H} \mathbf{~ N M R}\left(400 \mathrm{MHz}, \mathrm{CDCl}_{3}\right) \delta=7.40-7.34$ $(\mathrm{m}, 2 \mathrm{H}), 7.33-7.27(\mathrm{~m}, 2 \mathrm{H}), 7.25-7.19(\mathrm{~m}, 1 \mathrm{H}), 6.46(\mathrm{~d}, J=15.9 \mathrm{~Hz}, 1 \mathrm{H}), 6.19(\mathrm{dd}, J=15.9,9.0$ $\mathrm{Hz}, 1 \mathrm{H}), 3.70(\mathrm{~s}, 3 \mathrm{H}), 3.18-3.12(\mathrm{~m}, 1 \mathrm{H}), 1.91-1.78(\mathrm{~m}, 1 \mathrm{H}), 1.69-1.60(\mathrm{~m}, 1 \mathrm{H}), 1.39-1.27(\mathrm{~m}$, $4 \mathrm{H}), 0.89(\mathrm{t}, J=6.9 \mathrm{~Hz}, 3 \mathrm{H}) .{ }^{13} \mathbf{C} \mathbf{N M R}\left(100 \mathrm{MHz}, \mathrm{CDCl}_{3}\right) \delta=174.7,136.8,132.1,128.5,127.8$, 127.5, 126.3, 51.8, 49.6, 32.4, 29.3, 22.4, 13.9. HRMS (APCI) for $\mathrm{C}_{15} \mathrm{H}_{20} \mathrm{O}_{2}[\mathrm{M}]^{+}$: calcd 232.1458, found 232.1455 .

\subsection{Isomerization of Double Bond}

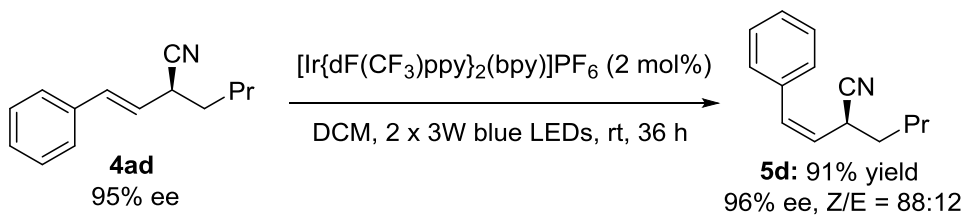

To a dried $10 \mathrm{~mL}$ Schlenk tube, 4ad (20.1 mg, $0.1 \mathrm{mmol}, 1.0$ equiv, $95 \%$ ee) and $\left[\operatorname{Ir}\left\{\mathrm{dF}\left(\mathrm{CF}_{3}\right)_{2} \mathrm{ppy}_{2}\right\}_{2}(\mathrm{bpy})\right] \mathrm{PF}_{6}(2.2 \mathrm{mg}, 0.002 \mathrm{mmol}, 2 \mathrm{~mol} \%)$ was dissolved in DCM (1.0 mL). Then, the resulting mixture was degassed via 'freeze-pumpthaw' procedure ( 3 times) under argon atmosphere. At last, the mixture was stirred at a distance of $\sim 1 \mathrm{~cm}$ from a $2 \times 3 \mathrm{~W}$ blue LEDs at $29^{\circ} \mathrm{C}$ about $36 \mathrm{~h}$. The mixture was then concentrated under reduced pressure and purified by flash column chromatography on silica gel to afford the mixture of $\mathbf{4 a d}$ and $\mathbf{5 d}(17.9 \mathrm{mg}, 91 \%$ yield, $\mathrm{Z} / \mathrm{E}=88: 12$, $96 \%$ ee) as light yellow oil, $[\alpha]_{\mathrm{D}}{ }^{25}=58.20\left(\mathrm{c}=0.66\right.$ in $\left.\mathrm{CHCl}_{3}\right)$; Ratio of $\mathbf{4 a d}$ and $\mathbf{5 d}$ was confirmed by ${ }^{1} \mathrm{H}$ NMR; ee was determined by HPLC analysis (Chiralpak AS-H column, hexane/i-PrOH, 95:5 v/v, 
flow rate $0.5 \mathrm{~mL} / \mathrm{min}, \lambda=220 \mathrm{~nm}, 25^{\circ} \mathrm{C}$ ), tR (major) $=9.63 \mathrm{~min}$, tR (minor) $=10.08 \mathrm{~min} ;{ }^{1} \mathbf{H ~ N M R}$ $\left(400 \mathrm{MHz}, \mathrm{CDCl}_{3}\right) \delta=7.43-7.35(\mathrm{~m}, 2 \mathrm{H}), 7.34-7.27(\mathrm{~m}, 1 \mathrm{H}), 7.27-7.21(\mathrm{~m}, 2 \mathrm{H}), 6.70(\mathrm{~d}, J=11.1$ $\mathrm{Hz}, 1 \mathrm{H}), 5.59(\mathrm{dd}, J=11.1,9.9 \mathrm{~Hz}, 1 \mathrm{H}), 3.65-3.58(\mathrm{~m}, 1 \mathrm{H}), 1.82-1.73(\mathrm{~m}, 1 \mathrm{H}), 1.68-1.59(\mathrm{~m}$, $1 \mathrm{H}), 1.51-1.37(\mathrm{~m}, 2 \mathrm{H}), 1.35-1.22(\mathrm{~m}, 2 \mathrm{H}), 0.88(\mathrm{t}, J=7.3 \mathrm{~Hz}, 3 \mathrm{H}) .{ }^{\mathbf{1 3}} \mathbf{C ~ N M R}\left(100 \mathrm{MHz}, \mathrm{CDCl}_{3}\right)$ $\delta=135.5,133.3,128.6,128.4,127.8,125.8,120.8,33.1,29.9,28.8,22.1,13.7$. HRMS (APCI) for $\mathrm{C}_{14} \mathrm{H}_{17} \mathrm{~N}[\mathrm{M}]^{+}$: calcd 199.1356, found 199.1369 .

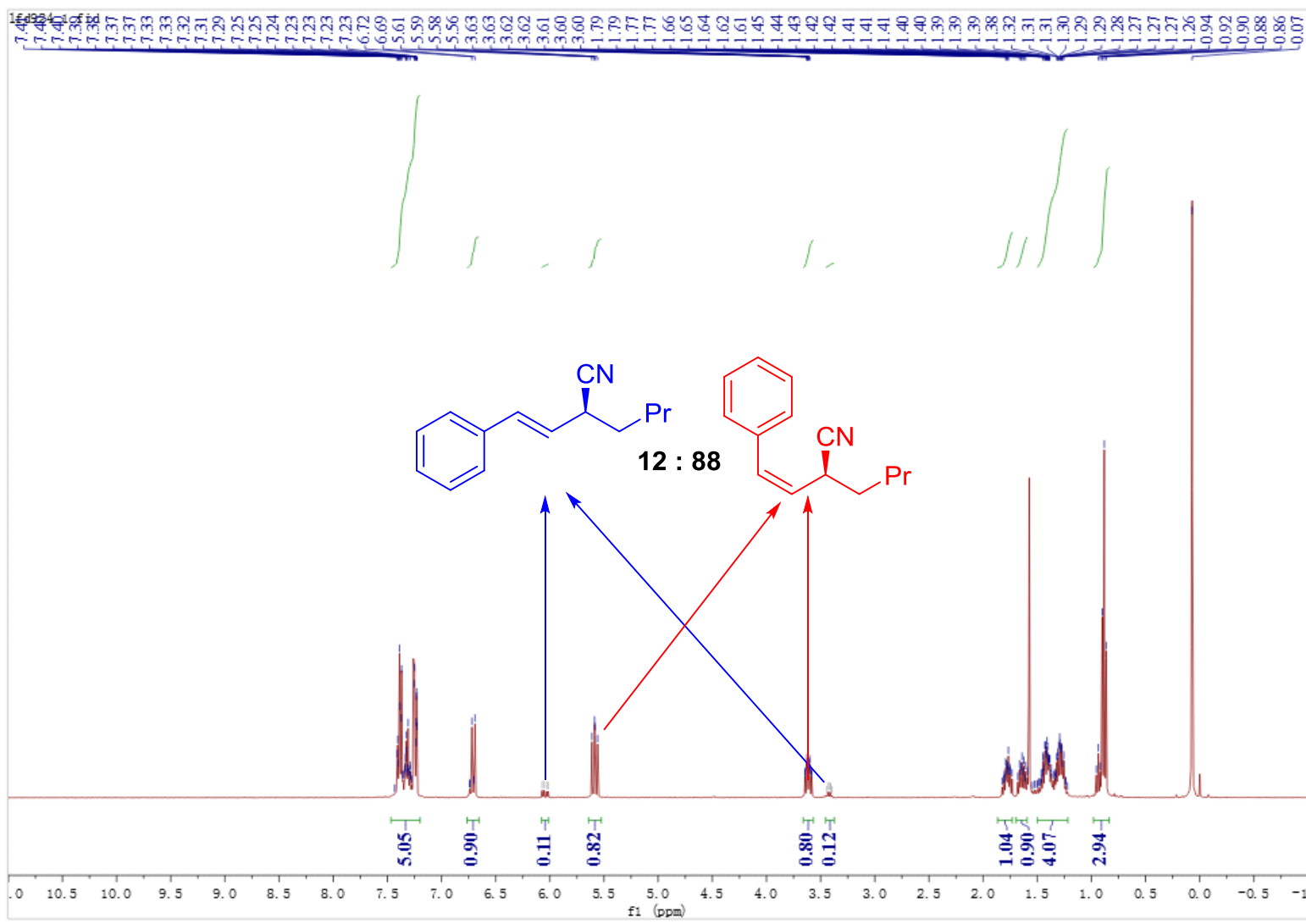

Figure S8. ${ }^{1} \mathrm{H}$ NMR spectrum of $\mathbf{4 a d}$ and $\mathbf{5 d}\left(400 \mathrm{MHz}, \mathrm{CDCl}_{3}\right)$ 


\section{Mechanistic Investigation}

\subsection{Control Experiment with Allyl Esters}
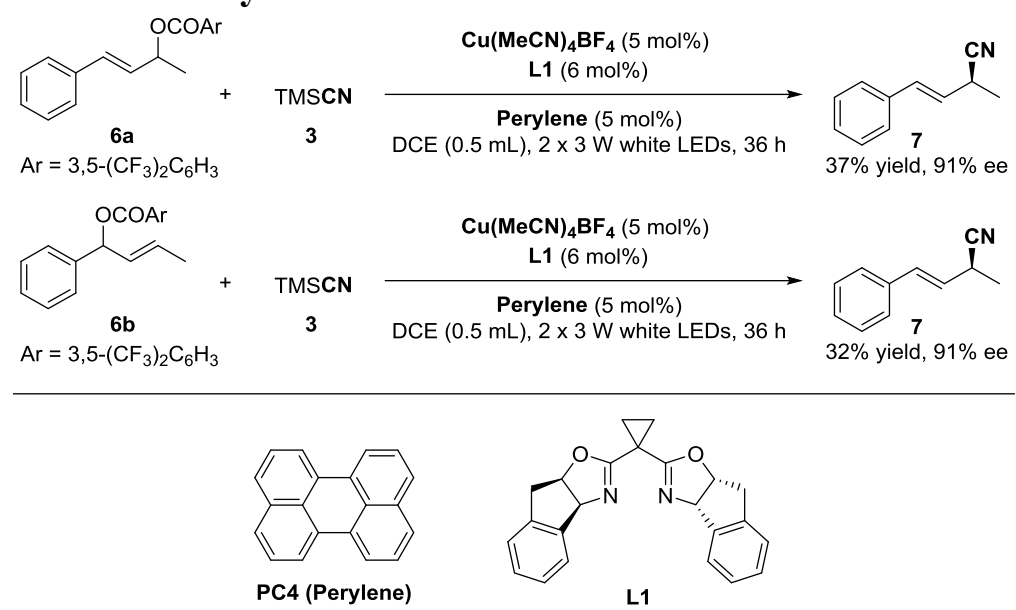

This reaction was conducted according to the general procedure C: In an argon-filled glove box, a flame-dried $10 \mathrm{ml}$ Schlenk tube equipped with a magnetic stirrer bar was charged sequentially with $\mathbf{C u}\left(\mathrm{CH}_{3} \mathbf{C N}\right)_{4} \mathbf{B F}_{4}(3.15 \mathrm{mg}, 0.01 \mathrm{mmol})$ and $\mathbf{L 1}(4.28 \mathrm{mg}, 0.012 \mathrm{mmol})$, followed by the addition of DCE $(0.5 \mathrm{~mL})$. The mixture was then stirred at room temperature for 30 minutes. Allyl ester $\mathbf{6 a} / \mathbf{6} \mathbf{b}$ (77.6 mg, $0.20 \mathrm{mmol}$ ), organo-photocatalyst Perylene (2.52 mg, $0.01 \mathrm{mmol}$ ) and TMSCN (59.5 mg, $76 \mu \mathrm{L}, 0.6 \mathrm{mmol}$ ) were added to the resulting mixture. The vial was closed and the Schlenk tube was removed from the glove box. At last, the mixture was stirred at a distance of $\sim 1 \mathrm{~cm}$ from a 2 x $3 \mathrm{~W}$ white LEDs at $29^{\circ} \mathrm{C}$ about $36 \mathrm{~h}$. The product was purified by flash column chromatography on silica gel to afford the desired product.

\section{$(S, E)$-2-Methyl-4-phenylbut-3-enenitrile (7)}

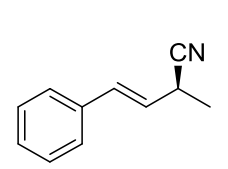

$37 \%(11.6 \mathrm{mg})$ isolated yield, light yellow oil, $[\alpha]_{\mathrm{D}}{ }^{25}=8.20\left(\mathrm{c}=0.38\right.$ in $\left.\mathrm{CHCl}_{3}\right) ; 91 \%$ ee, determined by HPLC analysis (Chiralpak AS-H column, hexane $/ i-\mathrm{PrOH}, 95: 5 \mathrm{v} / \mathrm{v}$, flow rate $0.5 \mathrm{~mL} / \mathrm{min}, \lambda=250 \mathrm{~nm}, 25^{\circ} \mathrm{C}$ ), tR (major) $=18.01 \mathrm{~min}$, $\mathrm{tR}$ (minor) $=16.58$ $\min ;{ }^{1} \mathbf{H}$ NMR $\left(400 \mathrm{MHz}, \mathrm{CDCl}_{3}\right) \delta=7.40-7.27(\mathrm{~m}, 5 \mathrm{H}), 6.72(\mathrm{dd}, J=15.8,1.5 \mathrm{~Hz}$, 1H), $6.07(\mathrm{dd}, J=15.8,6.1 \mathrm{~Hz}, 1 \mathrm{H}), 3.54-3.47(\mathrm{~m}, 1 \mathrm{H}), 1.51(\mathrm{~d}, J=7.2 \mathrm{~Hz}, 3 \mathrm{H}) .{ }^{13} \mathbf{C}$ NMR $(100$ $\mathrm{MHz}_{\mathrm{CDCl}}$ ) $\delta=135.7,132.5,128.7,128.2,126.5,124.3,120.9,28.4,19.0 . \mathrm{HRMS}$ (APCI) for $\mathrm{C}_{11} \mathrm{H}_{11} \mathrm{~N}[\mathrm{M}]^{+}$: calcd 157.0886, found 157.0890.

\subsection{Control Experiment with TEMPO}

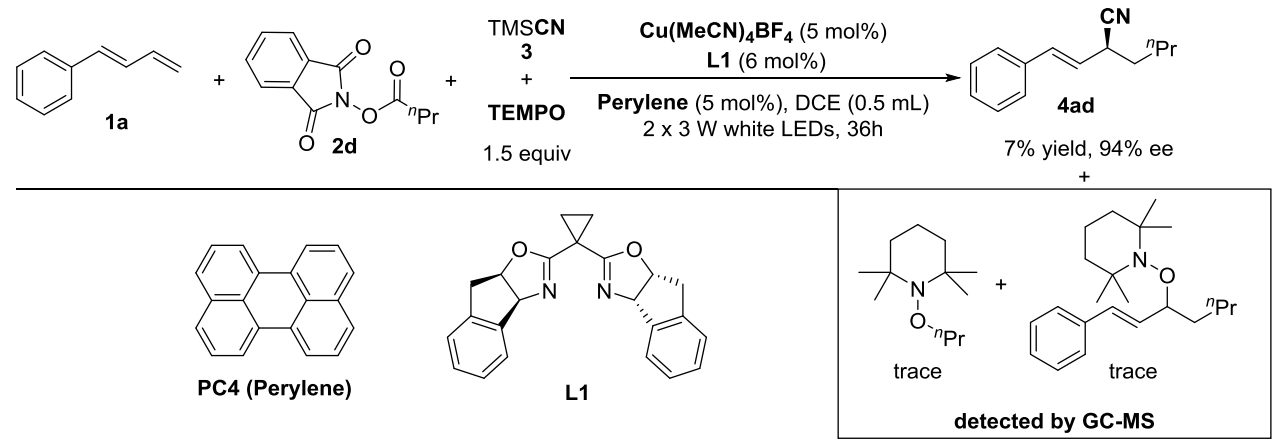


This reaction was conducted according to the general procedure C: In an argon-filled glove box, a flame-dried $10 \mathrm{ml}$ Schlenk tube equipped with a magnetic stirrer bar and rubber plug was charged sequentially with $\mathbf{C u}\left(\mathbf{C H}_{3} \mathbf{C N}\right)_{\mathbf{4}} \mathbf{B F}_{4}(3.15 \mathrm{mg}, 0.01 \mathrm{mmol})$ and $\mathbf{L 1}(4.28 \mathrm{mg}, 0.012 \mathrm{mmol})$, followed by the addition of DCE $(0.5 \mathrm{~mL})$. The mixture was then stirred at room temperature for 30 minutes. $\mathrm{N}$-(Acyloxy)phthalimides 2d $(46.6 \mathrm{mg}, 0.20 \mathrm{mmol})$, organo-photocatalyst Perylene $(2.52 \mathrm{mg}, 0.01$ mmol), TEMPO (46.9 mg, $0.3 \mathrm{mmol})$ and TMSCN $(59.5 \mathrm{mg}, 76 \mu \mathrm{L}, 0.6 \mathrm{mmol})$ were added to the resulting mixture. The vial was closed and the Schlenk tube was removed from the glove box. After that, 1,3-diene $1 \mathrm{a}(78.1 \mathrm{mg}, 84 \mu \mathrm{L}, 0.6 \mathrm{mmol})$ was added into the mixture through microinjector. At last, the mixture was stirred at a distance of $\sim 1 \mathrm{~cm}$ from a 2 x $3 \mathrm{~W}$ white LEDs at $29{ }^{\circ} \mathrm{C}$ about $36 \mathrm{~h}$.
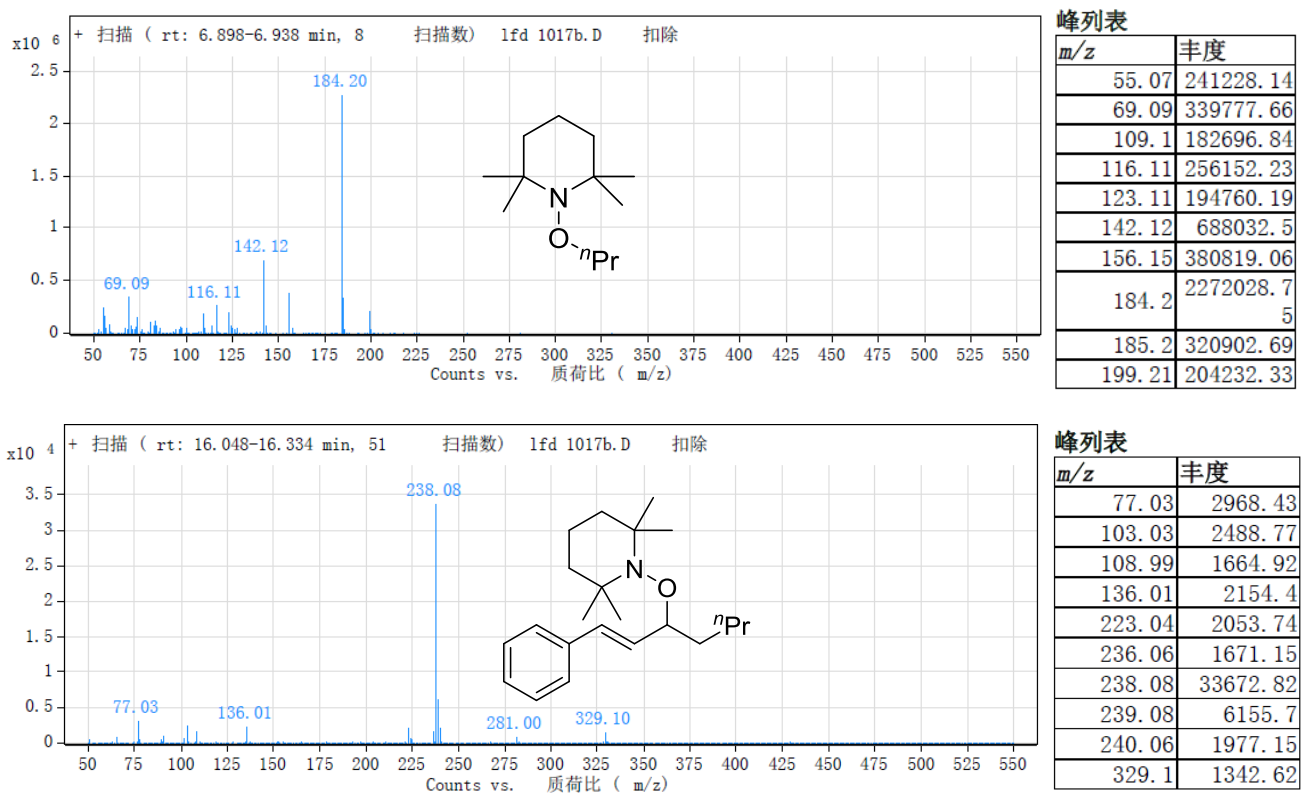

Figure S9. GC-MS spectrum of the TEMPO adducts

\subsection{Radical Clock Experiment}

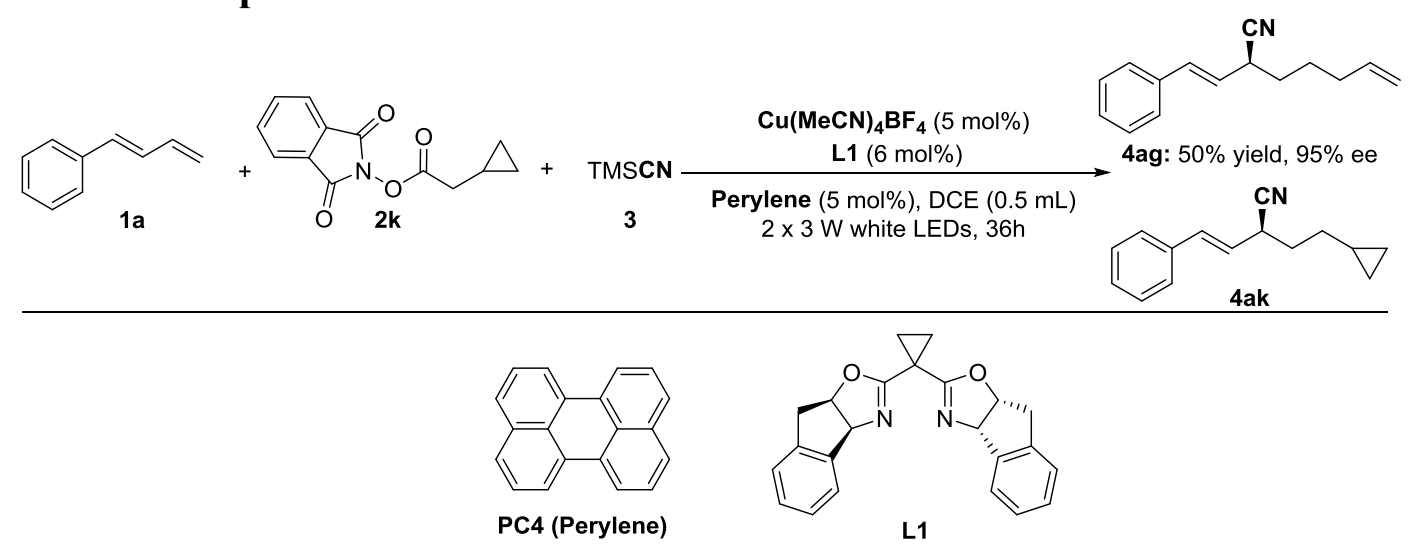

This reaction was conducted according to the general procedure $\mathrm{C}$, the reaction of $\mathbf{2 k}(49.0 \mathrm{mg}$, $0.2 \mathrm{mmol}$ ) gave $\mathbf{4 a g}$ (21.1 mg, 50\% yield, 95\% ee) as a light yellow oil, 4ak was not detected. 


\subsection{Light On-Off Experiments}
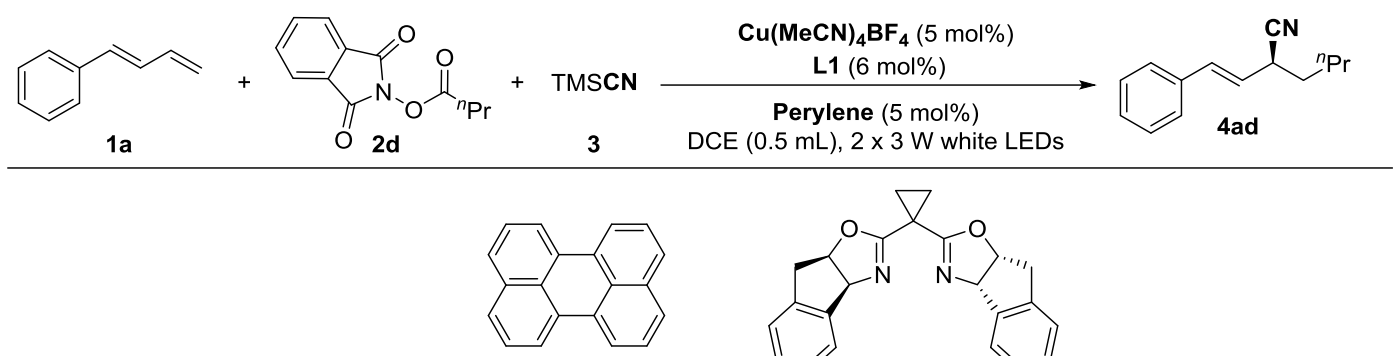

PC4 (Perylene)
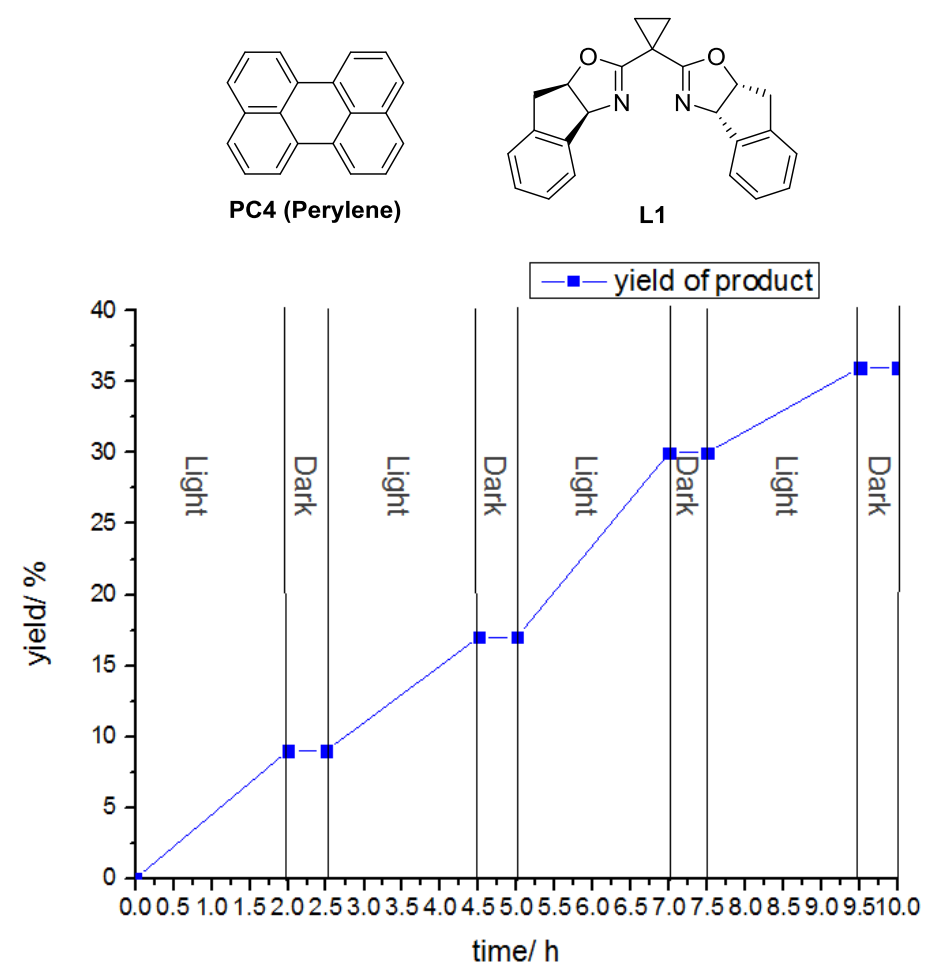

Figure S10. Light on-off experiments

The yield of 4ad was determined by ${ }^{1} \mathrm{H}$ NMR using 1,3,5-trimethoxybenzene as an internal standard. The results revealed that a radical chain process was not the major reaction pathway, while it could not be completely ruled out at the current stage.

\subsection{Determination of Quantum Yields}

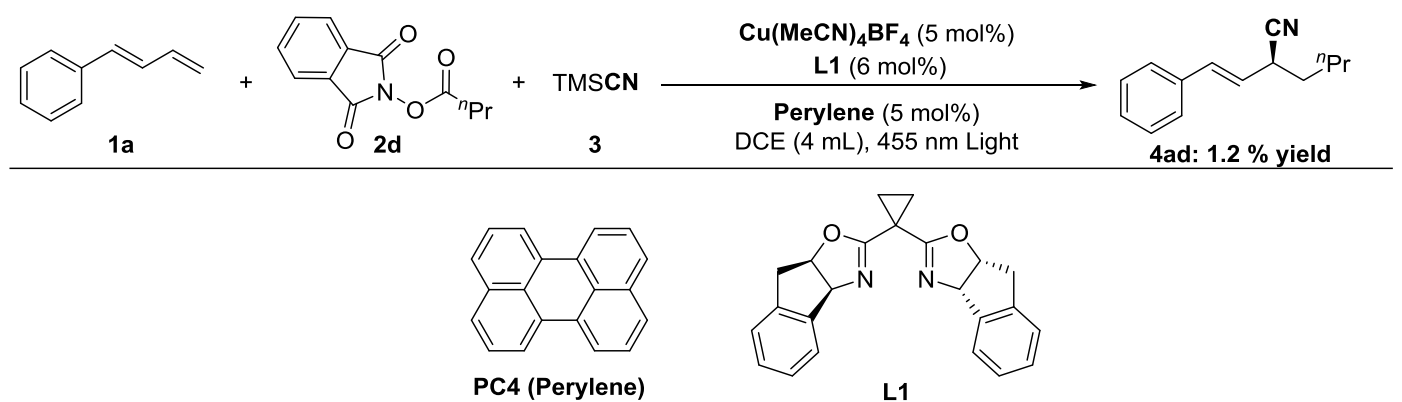

A cuvette was charged with $1 \mathrm{a}(1.2 \mathrm{mmol}), \mathbf{2 d}(0.4 \mathrm{mmol})$ and TMSCN $(1.2 \mathrm{mmol})$, $\mathrm{Cu}\left(\mathrm{CH}_{3} \mathrm{CN}\right)_{4} \mathbf{B F}_{4}(0.02 \mathrm{mmol})$ and $\mathbf{L 1}(0.024 \mathrm{mmol})$, Perylene $(0.02 \mathrm{mmol})$ and $4.0 \mathrm{~mL}$ DCE $(0.1$ M). The sample was irradiated $(\lambda=455 \mathrm{~nm}$, slit width $=3.0 \mathrm{~mm}$, slit height $5.0 \mathrm{~mm}$ with intensity of $\left.1.852 \mathrm{~mW} \cdot \mathrm{cm}^{-2}\right)$ for $18000 \mathrm{~s}(5 \mathrm{~h})$. The quantum yield was determined as follows. 
$\phi=$ Mole number for product/Mole number for absorption of photons $=\mathbf{0 . 2 7 7}$

$$
\phi=\frac{\mathrm{nN}_{\mathrm{A}} / \mathrm{t}}{\mathrm{fP} \lambda / \mathrm{hc}}
$$

$\mathrm{n}$ : the mole number of the product 4ad; t: reaction time $(18000 \mathrm{~s}, 5 \mathrm{~h})$; NA: $6.02 \times 10^{23} / \mathrm{mol}$; f: $1-10^{-\mathrm{A}}$ (455 nm, A= 1.05); P: P=E*S (E: illumination intensity, $E=1.852 \mathrm{~mW} / \mathrm{cm}^{2} ; \mathrm{S}$ : the area that irradiated $\left.\mathrm{S}=0.15 \mathrm{~cm}^{2}\right) ; \lambda$ : wavelength $\left(\lambda=4.55 \times 10^{-7} \mathrm{~m}\right) ; \mathrm{h}$ : planck constant $\left(\mathrm{h}=6.626 \times 10^{-34} \mathrm{~J} * \mathrm{~s}\right) ; \mathrm{c}$ : velocity of light $\left(\mathrm{c}=3 \times 10^{8} \mathrm{~m} / \mathrm{s}\right)$.

\subsection{Non-Linear Effect Experiments}

These reactions were conducted according to the general procedure C: In an argon-filled glove box, a flame-dried $10 \mathrm{ml}$ Schlenk tube equipped with a magnetic stirrer bar and rubber plug was charged sequentially with $\mathbf{C u}\left(\mathbf{C H}_{3} \mathbf{C N}\right)_{4} \mathbf{B F}_{4}(3.15 \mathrm{mg}, 0.01 \mathrm{mmol})$ and $\mathbf{L 1}$ (4.28 $\mathrm{mg}, 0.012 \mathrm{mmol}, \mathrm{x} \%$ ee), followed by the addition of DCE $(0.5 \mathrm{~mL})$. The mixture was then stirred at room temperature for 30 minutes. N-(Acyloxy)phthalimides $2 d$ ( $46.6 \mathrm{mg}, 0.20 \mathrm{mmol})$, organo-photocatalyst Perylene (2.52 $\mathrm{mg}, 0.01 \mathrm{mmol})$ and TMSCN $(59.5 \mathrm{mg}, 76 \mu \mathrm{L}, 0.6 \mathrm{mmol})$ were added to the resulting mixture. The vial was closed and the Schlenk tube was removed from the glove box. After that, 1,3-diene 1a (78.1 $\mathrm{mg}, 84 \mu \mathrm{L}, 0.6 \mathrm{mmol}$ ) was added into the mixture through microinjector. At last, the mixture was stirred at a distance of $\sim 1 \mathrm{~cm}$ from a $2 \times 3 \mathrm{~W}$ white LEDs at $29^{\circ} \mathrm{C}$ about $36 \mathrm{~h}$ until the reaction was completed, as monitored by TLC analysis.

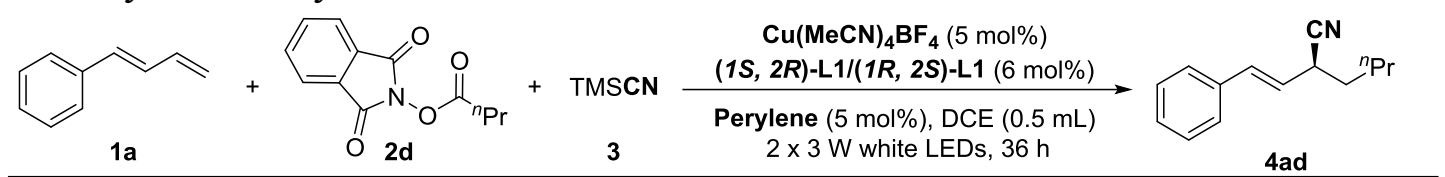

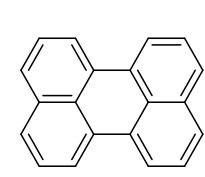

PC4 (Perylene)

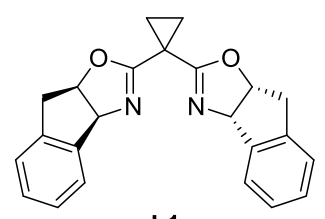

L1

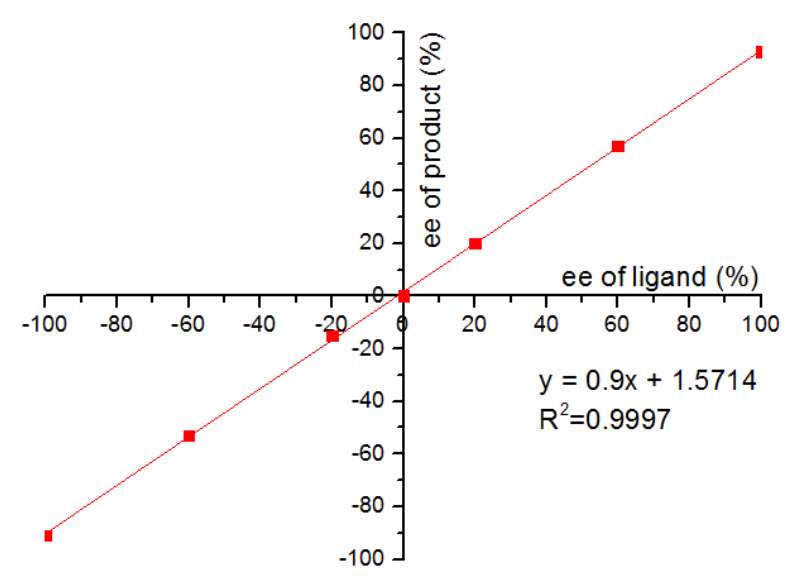

Figure S11. Relationship between ee values of ligand and product. 


\subsection{Luminescence Quenching Experiments}

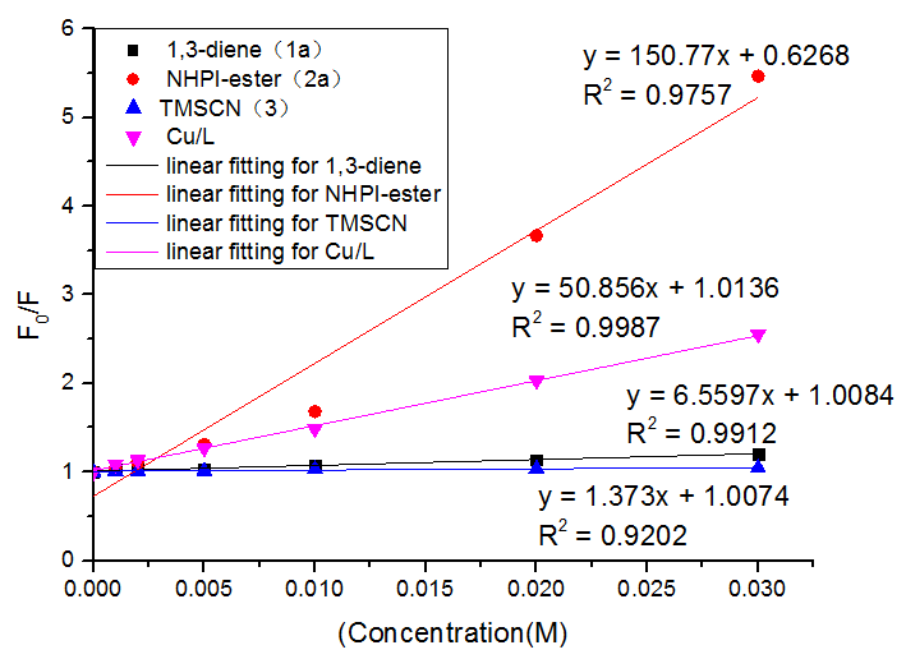

Figure S12. Perylene emission quenching by 1a, 2a, TMSCN and CuCN/L1.

Fluorescence spectra was collected on Agilent Fluorescence Spectrophotometer G9800A for all experiments. All perylene solutions were excited at $355 \mathrm{~nm}$ and the emission intensity was collected at $473 \mathrm{~nm}$. In a typical experiment, the emission spectrum of a $1 \times 10^{-4} \mathrm{M}$ solution of Perylene in DCE was collected. The significant decrease of Perylene luminescence could be observed in the presence of substrate 2a. And a slightly decrease of perylene luminescence was observed in the presence of 1a and TMSCN.

\subsection{IR Studies for Coordination Mode of Cyanide with Copper}

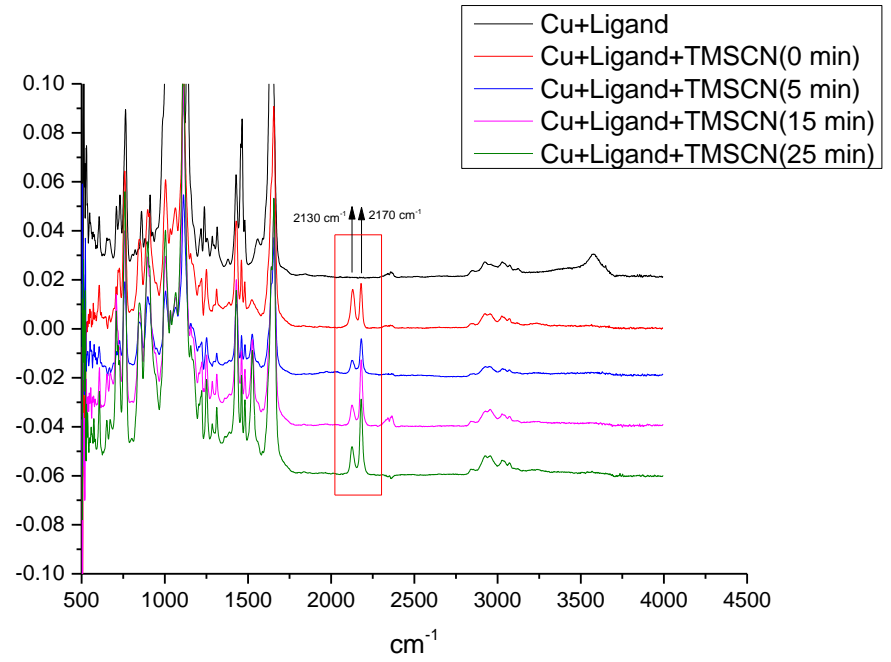

Figure S13. IR spectrum of $\mathrm{CuCN} / \mathrm{L} 1$ and TMSCN. 


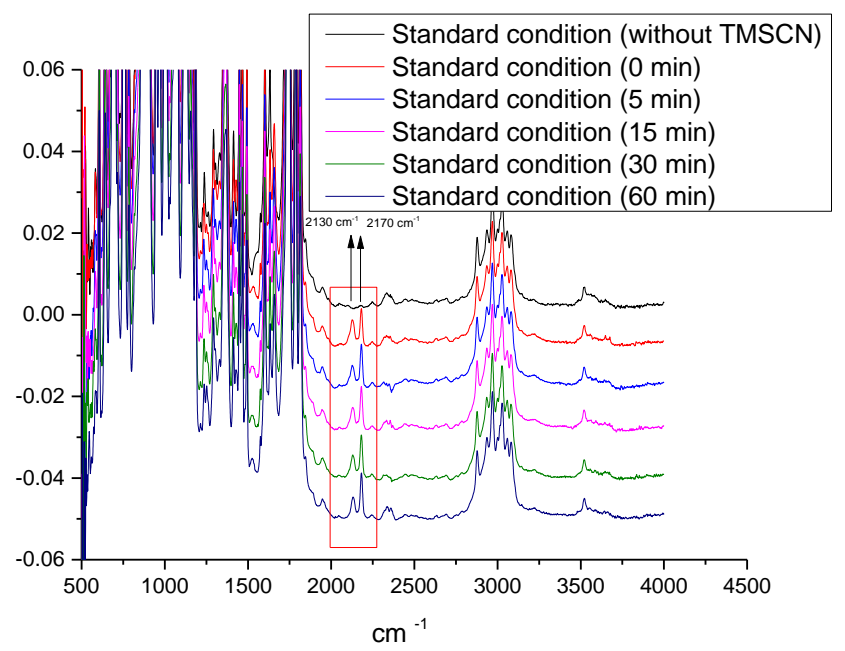

Figure S14. IR spectrum of reaction system.

IR spectra was collected on Thermo Scientific Nicolet iS50 FTIR for all experiments. When TMSCN $(76 \mu \mathrm{L}, 0.6 \mathrm{mmol})$ was added to $\mathrm{Cu} / \mathrm{L}$ solution $\left(\mathrm{Cu}\left(\mathrm{CH}_{3} \mathrm{CN}\right)_{4} \mathrm{BF}_{4}: 3.15 \mathrm{mg}, 0.01 \mathrm{mmol}\right.$; $\mathrm{L} 1: 4.28 \mathrm{mg}$, $0.012 \mathrm{mmol}$, DCE: $0.5 \mathrm{~mL}$ ), the characteristic signal of cyano carbon coordinate with copper (2130 $\mathrm{cm}^{-1}$ and $2170 \mathrm{~cm}^{-1}$ ) could be detected, but the signal of cyano nitrogen coordinate with copper (2088 $\mathrm{cm}^{-1}$ ) hasn't been detected. In addition, by monitoring the reaction system at different time, we still couldn't detect the characteristic signal of isocyanocopper species.

\subsection{Stereochemical Model for the Reaction of 4od}

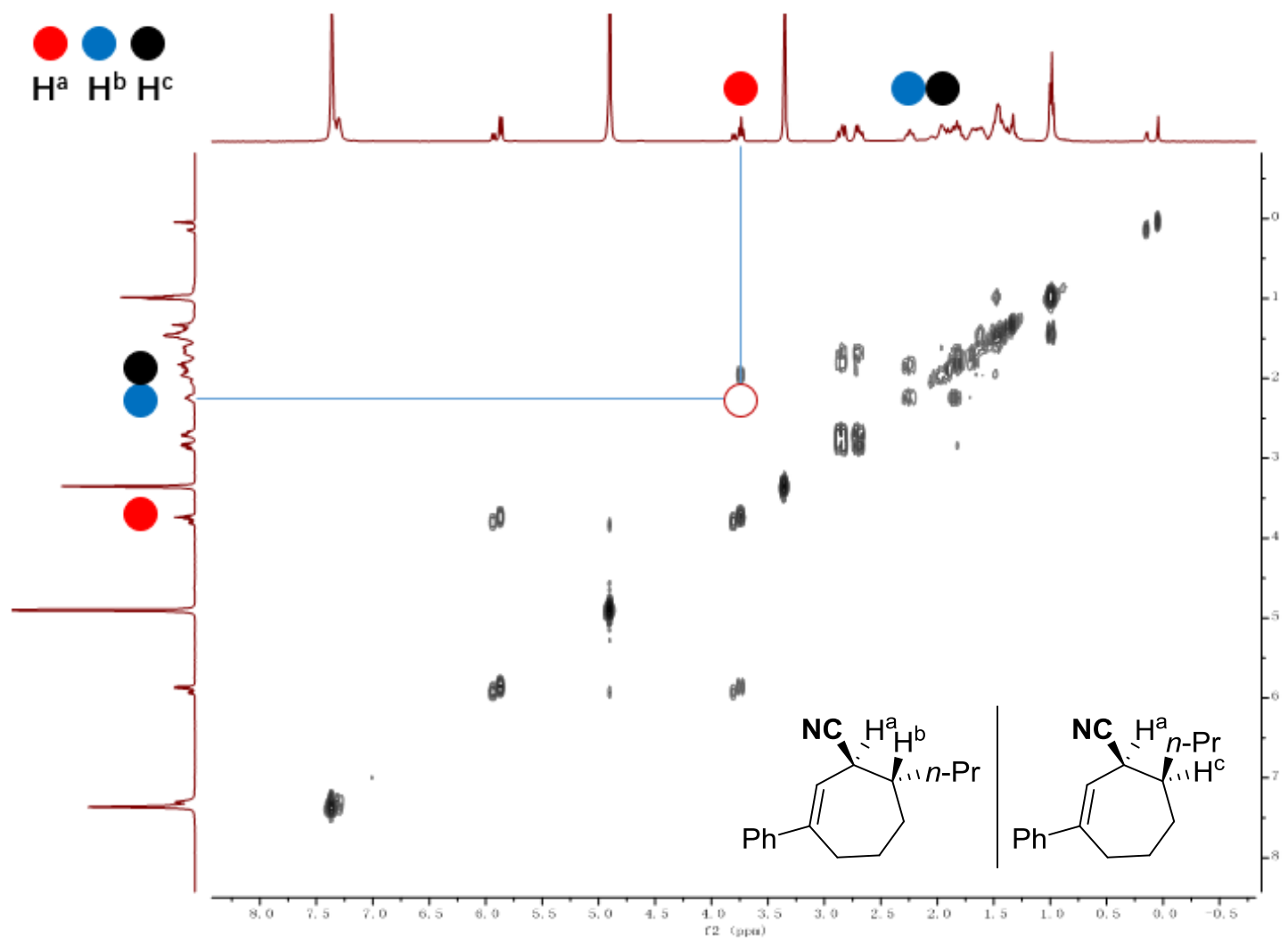

Figure S15. 2D-Cosy of product 4od 


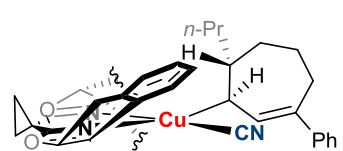

model A

$(1 S, 7 R)-40 d$ (major)

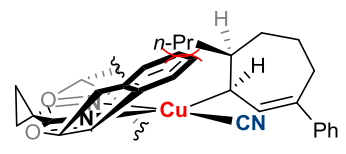

model B

$\prod$<smiles>N#C[C@@H]1C=C(c2ccccc2)CCC[C@H]1P</smiles>

(1S, 7S)-4od (minor)

Figure S16. Stereochemical model for the reaction of 4od

The relative configuration of two isomers of product 4od was confirmed by the 2D-Cosy spectrum. We thought that the propyl group in model B would repel with the benzene group of L1, making it difficult for the allyl radical to approach the $\mathrm{Cu}(\mathrm{II})$ intermediate, resulting in a diastereoselectivity (2.8:1).

\subsection{Byproduct Monitoring}
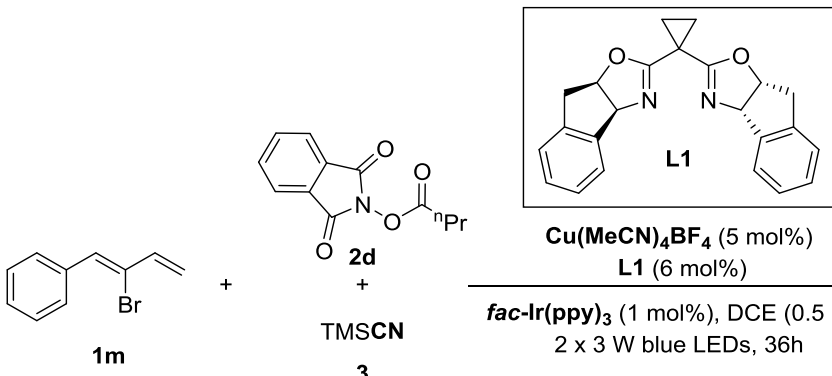

$$
\text { L1 (6 mol\%) }
$$

fac-Ir(ppy) $)_{3}(1 \mathrm{~mol} \%)$, DCE $(0.5 \mathrm{~mL})$ $2 \times 3 \mathrm{~W}$ blue LEDs, $36 \mathrm{~h}$
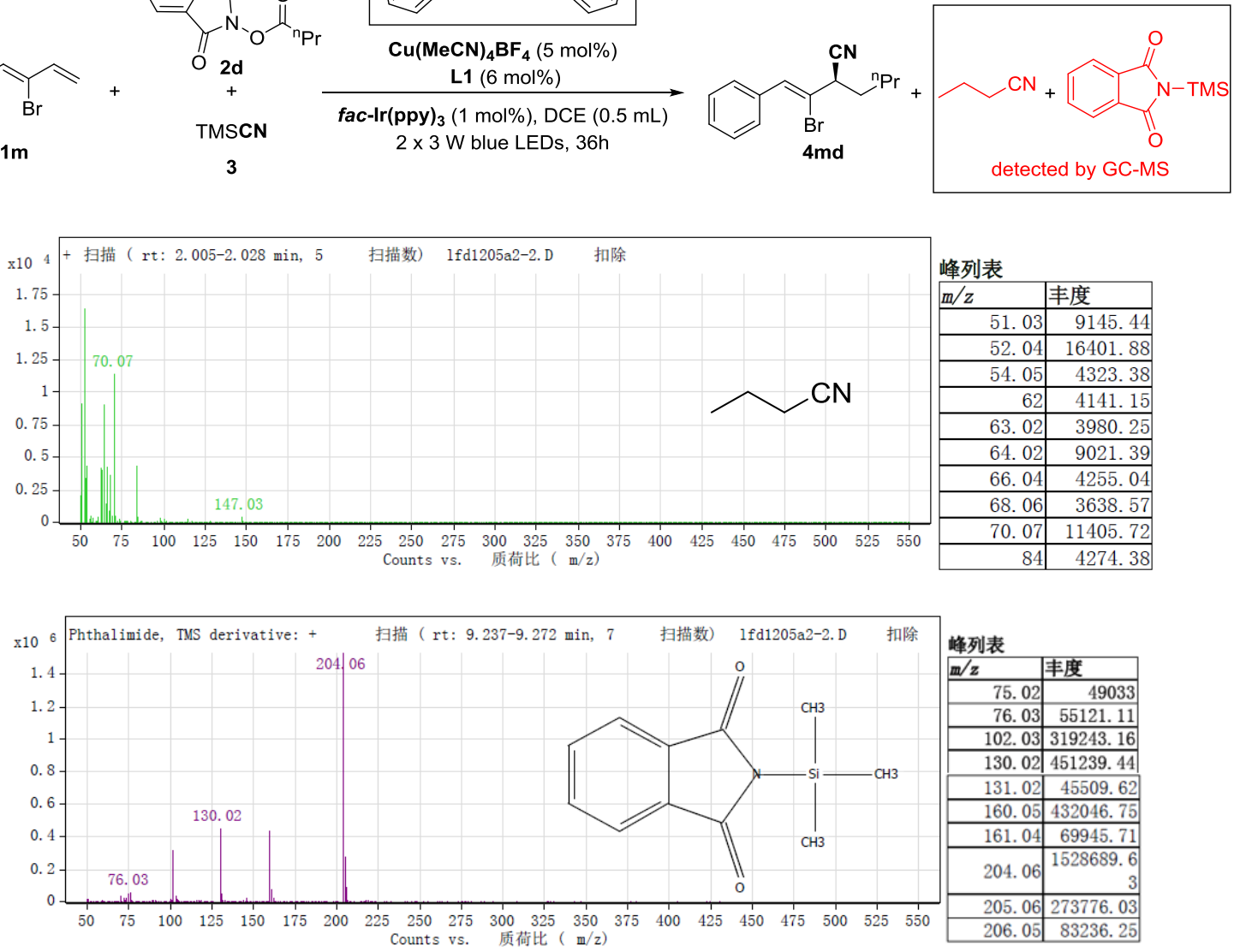

Figure S17. Byproduct monitoring for the reaction of $\mathbf{4 m d}$ 
The reaction mixture of the three-component carbocyanation for 4md was analyzed by GC-MS. The NHP ester was completely consumed, and byproduct butyronitrile and TMS-NHP were observed by GC-MS. According to this result, we thought the low yield may be the result of competitive twocomponent couplings between alkyl radicals with TMSCN or other unknown off-cycle reaction pathways. Even so, more mechanism studies are still needed in the following work.

\section{Extensions of reaction methodology}

\subsection{Enantioselective Difunctionalization of 1,3-Enynes}

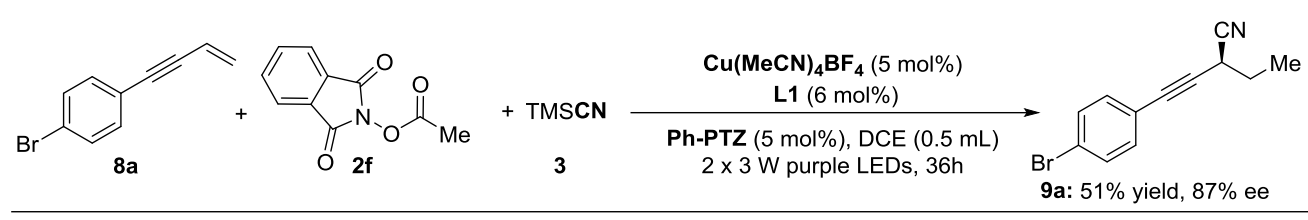

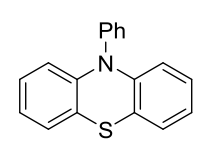

PC1 (Ph-PTZ)

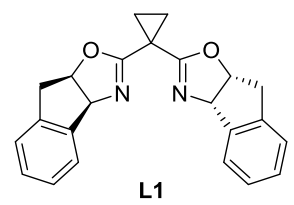

This reaction was conducted according to the general procedure $\mathrm{C}$ : In an argon-filled glove box, a flame-dried $10 \mathrm{ml}$ Schlenk tube equipped with a magnetic stirrer bar and rubber plug was charged sequentially with $\mathbf{C u}\left(\mathbf{C H}_{3} \mathbf{C N}\right)_{\mathbf{4}} \mathbf{B F}_{4}(3.15 \mathrm{mg}, 0.01 \mathrm{mmol})$ and $\mathbf{L 1}(4.28 \mathrm{mg}, 0.012 \mathrm{mmol})$, followed by the addition of DCE $(0.5 \mathrm{~mL})$. The mixture was then stirred at room temperature for 30 minutes. $N$-(Acyloxy)phthalimides $2 \mathbf{f}(0.20 \mathrm{mmol})$, organo-photocatalyst Ph-PTZ $(0.01 \mathrm{mmol})$ and TMSCN $(0.6 \mathrm{mmol})$ were added to the resulting mixture. The vial was closed and the Schlenk tube was removed from the glove box. After that, 1,3-enyne 8a $(0.6 \mathrm{mmol})$ was added into the mixture through microinjector. At last, the mixture was stirred at a distance of $\sim 1 \mathrm{~cm}$ from a $2 \times 3 \mathrm{~W}$ purple LEDs at $29{ }^{\circ} \mathrm{C}$ about $36 \mathrm{~h}$ until the reaction was completed, as monitored by TLC analysis. The product was purified by flash column chromatography on silica gel to afford the desired product.

\section{(S)-4-(4-Bromophenyl)-2-ethylbut-3-ynenitrile (9a)}

$$
\begin{aligned}
& 51 \%(25.5 \mathrm{mg}) \text { isolated yield, light yellow oil, }[\alpha]_{\mathrm{D}}^{25}=-8.47\left(\mathrm{c}=0.84 \mathrm{in} \mathrm{CHCl}_{3}\right) \text {; } \\
& 87 \% \text { ee, determined by HPLC analysis (Chiralpak AS-H column, hexane } i \text { - } \mathrm{PrOH}, \\
& \left.99: 1 \mathrm{v} / \mathrm{v}, \text { flow rate } 1 \mathrm{~mL} / \mathrm{min}, \lambda=254 \mathrm{~nm}, 25^{\circ} \mathrm{C}\right), \mathrm{tR}(\text { major })=20.39 \mathrm{~min}, \mathrm{tR}(\text { minor) } \\
& =19.12 \mathrm{~min} ;{ }^{1} \mathbf{H ~ N M R ~}\left(400 \mathrm{MHz}, \mathrm{CDCl}_{3}\right) \delta=7.46(\mathrm{~d}, J=8.2 \mathrm{~Hz}, 2 \mathrm{H}), 7.30(\mathrm{~d}, J
\end{aligned}
$$
$=8.3 \mathrm{~Hz}, 2 \mathrm{H}), 3.70(\mathrm{t}, J=6.6 \mathrm{~Hz}, 1 \mathrm{H}), 2.02-1.96(\mathrm{~m}, 2 \mathrm{H}), 1.21(\mathrm{t}, J=7.4 \mathrm{~Hz}, 3 \mathrm{H}) .{ }^{\mathbf{1 3}} \mathbf{C} \mathbf{N M R}(100$ $\left.\mathrm{MHz}_{2} \mathrm{CDCl}_{3}\right) \delta=133.2,131.7,123.2,120.7,117.3,83.0,82.2,26.9,25.3,11.2$. HRMS (APCI) for $\mathrm{C}_{12} \mathrm{H}_{10} \mathrm{BrN}[\mathrm{M}]^{+}$: calcd 246.9991, found 246.9995. 


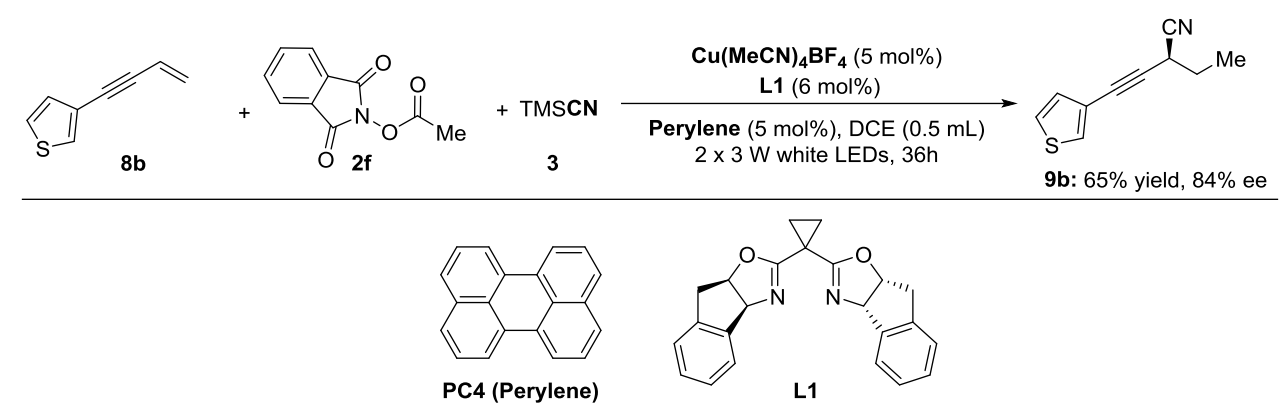

This reaction was conducted according to the general procedure C: In an argon-filled glove box, a flame-dried $10 \mathrm{ml}$ Schlenk tube equipped with a magnetic stirrer bar and rubber plug was charged sequentially with $\mathbf{C u}\left(\mathbf{C H}_{3} \mathbf{C N}\right)_{4} \mathbf{B F}_{4}(3.15 \mathrm{mg}, 0.01 \mathrm{mmol})$ and $\mathbf{L 1}(4.28 \mathrm{mg}, 0.012 \mathrm{mmol})$, followed by the addition of DCE $(0.5 \mathrm{~mL})$. The mixture was then stirred at room temperature for 30 minutes. $N$-(Acyloxy)phthalimides $2 \mathbf{f}(0.20 \mathrm{mmol})$, organo-photocatalyst Perylene $(0.01 \mathrm{mmol})$ and TMSCN $(0.6 \mathrm{mmol})$ were added to the resulting mixture. The vial was closed and the Schlenk tube was removed from the glove box. After that, 1,3-enyne $8 \mathrm{~b}(0.6 \mathrm{mmol})$ was added into the mixture through microinjector. At last, the mixture was stirred at a distance of $\sim 1 \mathrm{~cm}$ from a $2 \times 3 \mathrm{~W}$ white LEDs at 29 ${ }^{\circ} \mathrm{C}$ about $36 \mathrm{~h}$ until the reaction was completed, as monitored by TLC analysis. The product was purified by flash column chromatography on silica gel to afford the desired product.

(S)-2-Ethyl-4-(thiophen-3-yl)but-3-ynenitrile (9b)

$65 \%(22.7 \mathrm{mg})$ isolated yield, light yellow oil, $[\alpha]_{\mathrm{D}} 25=0.67\left(\mathrm{c}=0.65 \mathrm{in} \mathrm{CHCl}_{3}\right) ; 84 \%$
ee, determined by HPLC analysis (Chiralpak AS-H column, hexane/i-PrOH, 99:1 v/v,
flow rate $\left.1 \mathrm{~mL} / \mathrm{min}, \lambda=254 \mathrm{~nm}, 25^{\circ} \mathrm{C}\right), \mathrm{tR}($ major $)=22.05 \mathrm{~min}, \mathrm{tR}(\mathrm{minor})=19.27$
$\min ;{ }^{1} \mathbf{H ~ N M R}\left(400 \mathrm{MHz}, \mathrm{CDCl}_{3}\right) \delta=7.48(\mathrm{dd}, J=2.9,1.2 \mathrm{~Hz}, 1 \mathrm{H}), 7.27(\mathrm{dd}, J=5.4$, $2.5 \mathrm{~Hz}, 1 \mathrm{H}), 7.11(\mathrm{dd}, J=5.0,1.2 \mathrm{~Hz}, 1 \mathrm{H}), 3.70(\mathrm{t}, J=6.6 \mathrm{~Hz}, 1 \mathrm{H}), 2.02-1.95(\mathrm{~m}, 2 \mathrm{H}), 1.21(\mathrm{t}, J=$ $7.4 \mathrm{~Hz}, 3 \mathrm{H}) .{ }^{13} \mathbf{C} \mathbf{~ N M R}\left(100 \mathrm{MHz}, \mathrm{CDCl}_{3}\right) \delta=129.7,125.5,120.7,117.5,80.6,79.3,27.0,25.2,11.2$. HRMS (EI) for $\mathrm{C}_{10} \mathrm{H}_{9} \mathrm{NS}[\mathrm{M}]^{+}$: calcd 175.0450, found calcd 175.0460.

\subsection{Enantioselective Carbonylative Difunctionalizations}
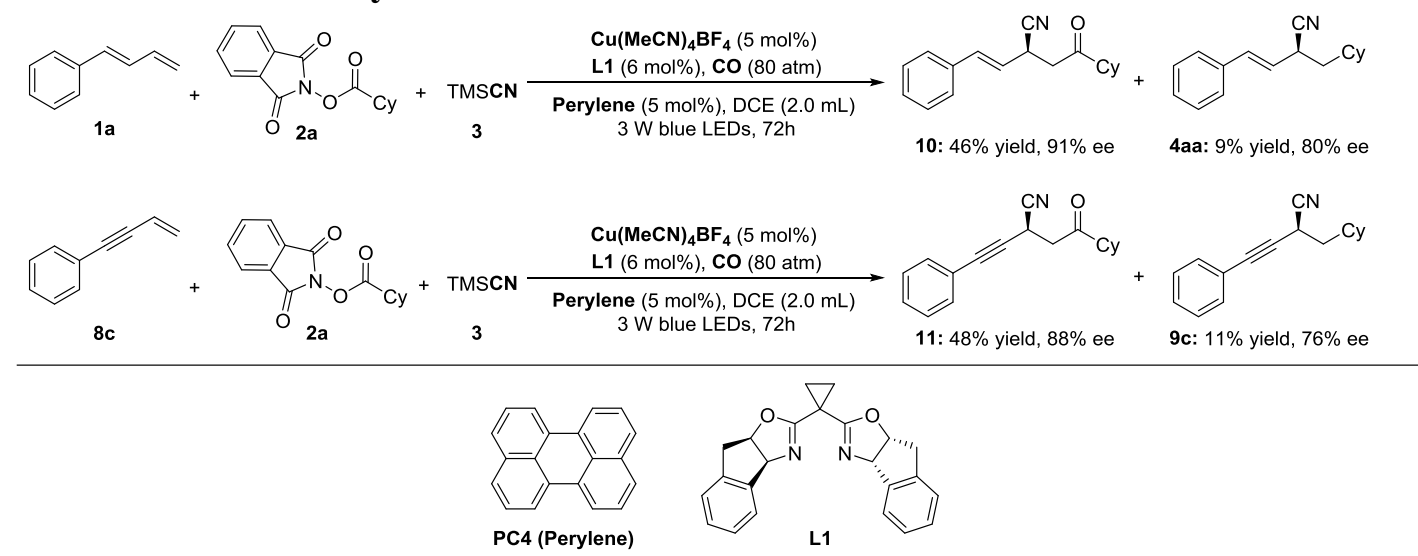

General procedure D: In an argon-filled glove box, a flame-dried $4 \mathrm{ml}$ glass bottle equipped with a magnetic stirrer bar was charged sequentially with $\mathbf{C u}\left(\mathbf{C H}_{3} \mathbf{C N}\right)_{4} \mathbf{B F}_{4}(3.15 \mathrm{mg}, 0.01 \mathrm{mmol})$ and $\mathbf{L 1}$ $(4.28 \mathrm{mg}, 0.012 \mathrm{mmol})$, followed by the addition of DCE $(0.5 \mathrm{~mL})$. The mixture was then stirred at room temperature for 30 minutes. $N$-(Acyloxy)phthalimides $2 \mathrm{a}(54.6 \mathrm{mg}, 0.20 \mathrm{mmol})$, organophotocatalyst Perylene (2.52 mg, $0.01 \mathrm{mmol})$ and TMSCN $(59.5 \mathrm{mg}, 76 \mu \mathrm{L}, 0.6 \mathrm{mmol})$ were added to 
the resulting mixture. The vial was closed and removed from the glove box. After that, 1,3-diene 1a or 1,3-enyne $8 \mathbf{c}(0.6 \mathrm{mmol})$ was added into the mixture through microinjector. The glass bottle was inserted in a needle and placed in the high pressure photoreactor, which was exchanged with $\mathrm{CO}$ for three times and kept at $80 \mathrm{~atm} \mathrm{CO}$. At last, the mixture was stirred at a distance of $\sim 1 \mathrm{~cm}$ from a $3 \mathrm{~W}$ blue LEDs about $72 \mathrm{~h}$ until the reaction was completed. The product was purified by flash column chromatography on silica gel to afford the desired product.

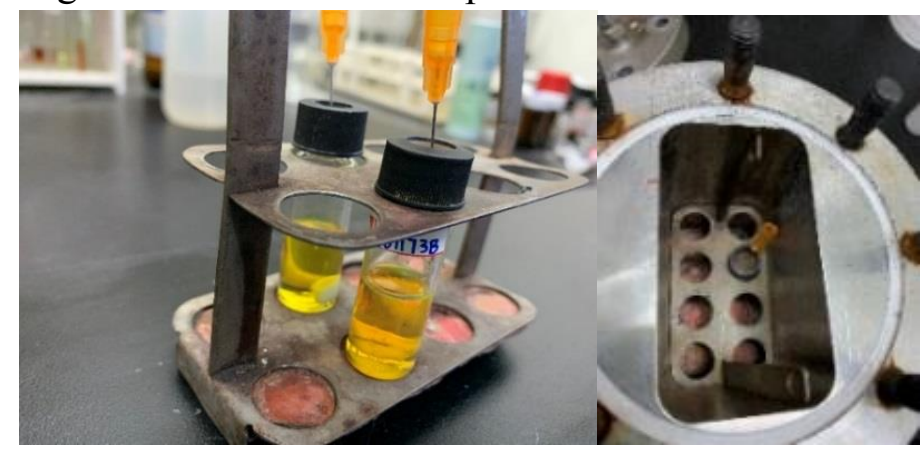

Figure S18. $4 \mathrm{~mL}$ glass bottle for carbonylative difunctionalization

(S, E)-2-(2-Cyclohexyl-2-oxoethyl)-4-phenylbut-3-enenitrile (10)

$46 \%(24.4 \mathrm{mg})$ isolated yield, colorless oil, $[\alpha]_{\mathrm{D}}^{25}=1.96\left(\mathrm{c}=0.81 \mathrm{in} \mathrm{CHCl}_{3}\right) ; 91 \%$
$\begin{aligned} & \text { ee, determined by HPLC analysis }(\text { Chiralpak AD-H column, hexane } / i-\mathrm{PrOH}, 90: 10 \\ & \left.\mathrm{v} / \mathrm{v}, \text { flow rate } 1.0 \mathrm{~mL} / \mathrm{min}, \lambda=254 \mathrm{~nm}, 25^{\circ} \mathrm{C}\right), \mathrm{tR} \text { (major) }=10.84 \mathrm{~min}, \mathrm{tR}(\mathrm{minor})\end{aligned}$ $=8.95 \mathrm{~min} ;{ }^{1} \mathbf{H} \mathbf{N M R}\left(400 \mathrm{MHz}, \mathrm{CDCl}_{3}\right) \delta=\delta 7.37-7.27(\mathrm{~m}, 5 \mathrm{H}), 6.77(\mathrm{dd}, J=15.9,1.5 \mathrm{~Hz}, 1 \mathrm{H})$, $6.04(\mathrm{dd}, J=15.8,6.2 \mathrm{~Hz}, 1 \mathrm{H}), 4.00-3.95(\mathrm{~m}, 1 \mathrm{H}), 3.02(\mathrm{dd}, J=17.8,6.7 \mathrm{~Hz}, 1 \mathrm{H}), 2.86(\mathrm{dd}, J=17.9$, $7.1 \mathrm{~Hz}, 1 \mathrm{H}), 2.40-2.32(\mathrm{~m}, 1 \mathrm{H}), 1.92-1.76(\mathrm{~m}, 4 \mathrm{H}), 1.69-1.67(\mathrm{~m}, 1 \mathrm{H}), 1.41-1.21(\mathrm{~m}, 5 \mathrm{H}) .{ }^{13} \mathrm{C}$ $\operatorname{NMR}\left(100 \mathrm{MHz}, \mathrm{CDCl}_{3}\right) \delta=208.6,135.4,133.6,128.7,128.3,126.5,121.8,119.8,50.6,43.5,28.8$, 28.1, 25.6, 25.4. HRMS (ESI) for $\mathrm{C}_{18} \mathrm{H}_{21} \mathrm{NNaO}[\mathrm{M}+\mathrm{Na}]^{+}$: calcd 290.1515, found 290.1518.

\section{(S)-2-(2-Cyclohexyl-2-oxoethyl)-4-phenylbut-3-ynenitrile (11)}

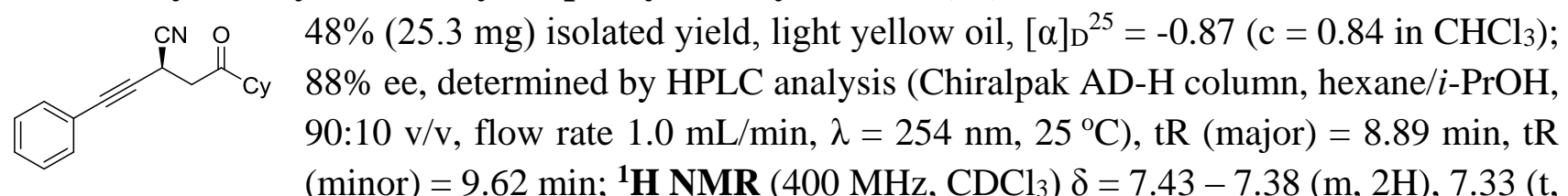
$J=5.2 \mathrm{~Hz}, 4 \mathrm{H}), 4.27(\mathrm{t}, J=7.0 \mathrm{~Hz}, 1 \mathrm{H}), 3.21-3.03(\mathrm{~m}, 2 \mathrm{H}), 2.44-2.36(\mathrm{~m}, 1 \mathrm{H}), 1.94-1.77(\mathrm{~m}$, $4 \mathrm{H}), 1.68(\mathrm{dd}, J=11.6,4.8 \mathrm{~Hz}, 1 \mathrm{H}), 1.45-1.17(\mathrm{~m}, 5 \mathrm{H}) .{ }^{13} \mathbf{C ~ N M R}\left(100 \mathrm{MHz}, \mathrm{CDCl}_{3}\right) \delta=207.5$, $131.8,129.0,128.3,121.4,117.2,83.9,80.5,50.5,43.6,28.1,28.0,25.6,25.4,25.3,18.7$. HRMS (ESI) for $\mathrm{C}_{18} \mathrm{H}_{19} \mathrm{NNaO}[\mathrm{M}+\mathrm{Na}]^{+}$: calcd 288.1359, found 288.1358.

\section{(S)-2-(Cyclohexylmethyl)-4-phenylbut-3-ynenitrile (9c)}

$\mathrm{CN}_{\mathrm{C}} 11 \%(5.2 \mathrm{mg})$ isolated yield, light yellow oil, $[\alpha]_{\mathrm{D}}^{25}=-0.70\left(\mathrm{c}=0.52 \mathrm{in} \mathrm{CHCl}_{3}\right) ; 76 \%$ ee, determined by HPLC analysis (Chiralpak AD-H column, hexane/i-PrOH, 99:1 $\mathrm{v} / \mathrm{v}$, flow rate $0.5 \mathrm{~mL} / \mathrm{min}, \lambda=254 \mathrm{~nm}, 25^{\circ} \mathrm{C}$ ), $\mathrm{tR}$ (major) $=11.49 \mathrm{~min}, \mathrm{tR}$ (minor) $=$ $11.98 \mathrm{~min} ;{ }^{1} \mathbf{H} \mathbf{N M R}\left(400 \mathrm{MHz}, \mathrm{CDCl}_{3}\right) \delta=7.43(\mathrm{dd}, \mathrm{J}=7.6,2.1 \mathrm{~Hz}, 2 \mathrm{H}), 7.36-$ $7.29(\mathrm{~m}, 3 \mathrm{H}), 3.81(\mathrm{t}, J=7.8 \mathrm{~Hz}, 1 \mathrm{H}), 1.92-1.60(\mathrm{~m}, 9 \mathrm{H}), 1.34-1.24(\mathrm{~m}, 3 \mathrm{H}), 1.02-0.91(\mathrm{~m}, 2 \mathrm{H})$. ${ }^{13} \mathrm{C} \mathrm{NMR}\left(100 \mathrm{MHz}, \mathrm{CDCl}_{3}\right) \delta=131.7,128.8,128.3,121.8,117.9,83.7,81.4,40.5,35.0,32.5,26.2$, 25.8, 21.4. HRMS (ESI) for $\mathrm{C}_{17} \mathrm{H}_{20} \mathrm{~N}[\mathrm{M}+\mathrm{H}]^{+}$: calcd 238.1590, found 238.1573. 


\section{Copies of NMR Spectra}

${ }^{1} \mathrm{H}$ NMR spectrum of compound $4 \mathrm{aa}\left(400 \mathrm{MHz} \mathrm{CDCl}_{3}\right)$

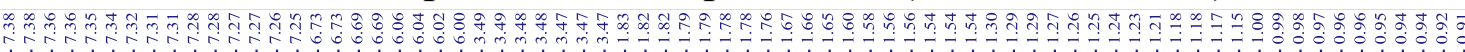

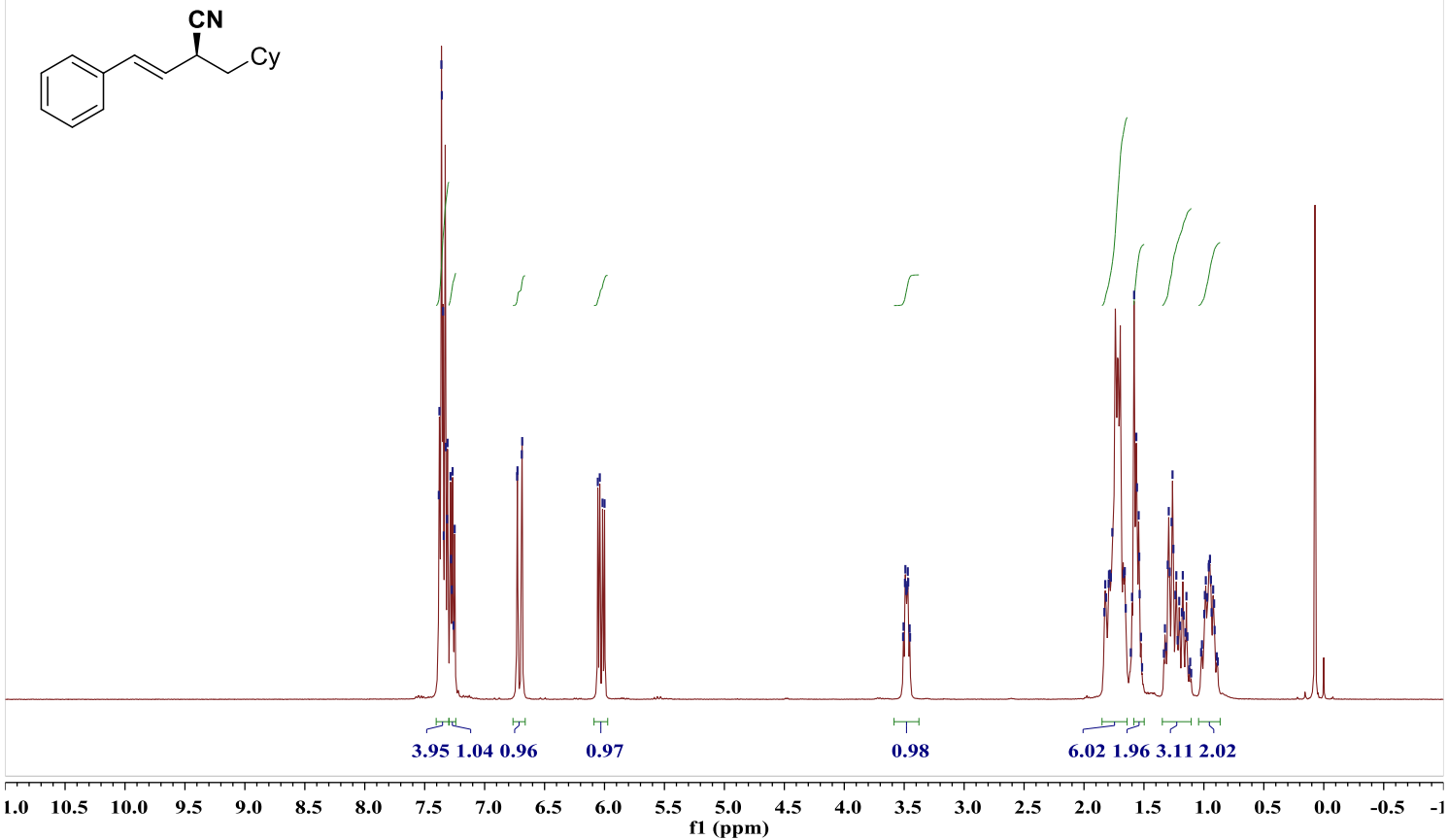

${ }^{13} \mathrm{C}$ NMR spectrum of compound $4 \mathrm{aa}\left(100 \mathrm{MHz}, \mathrm{CDCl}_{3}\right)$

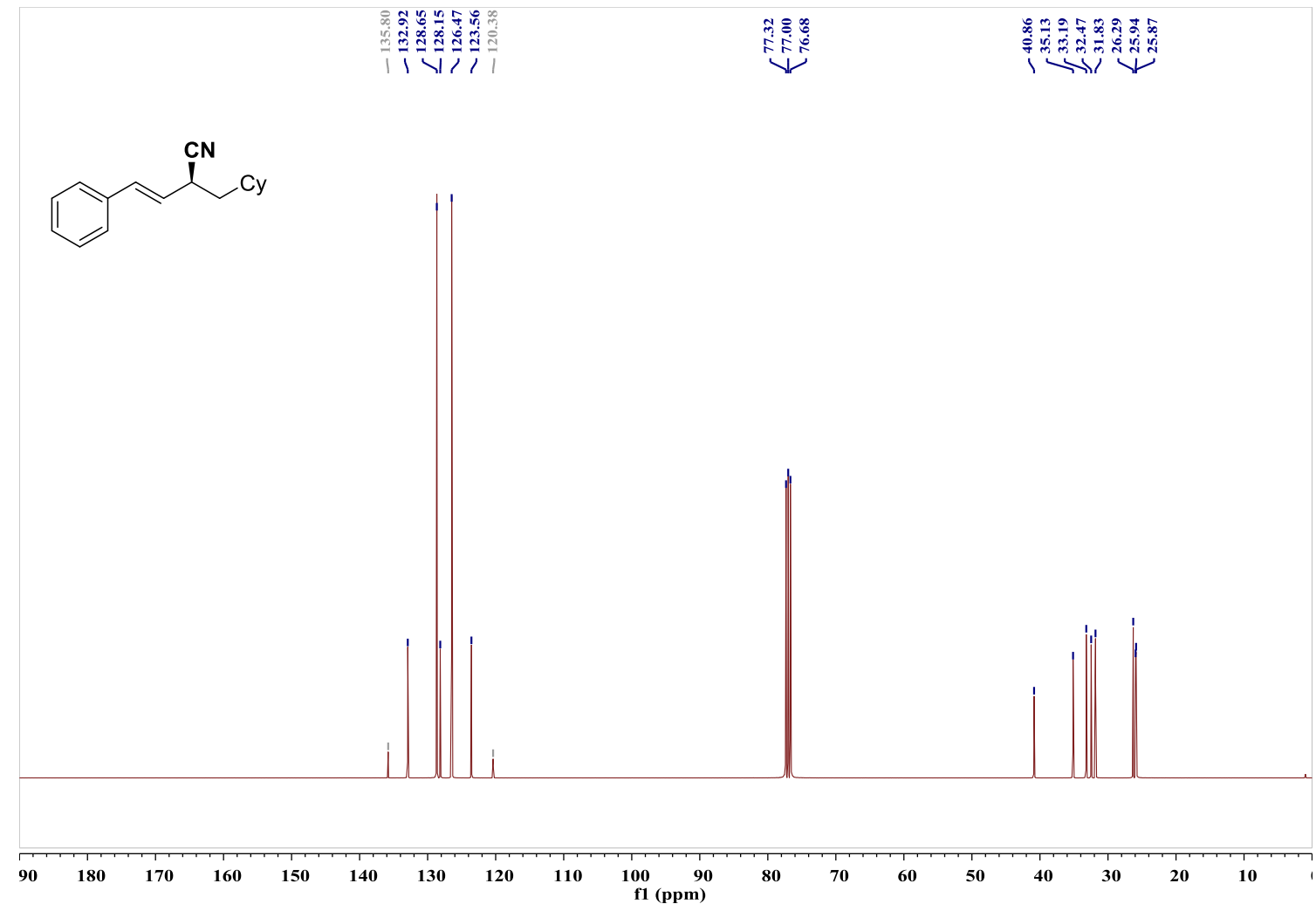


${ }^{1} \mathrm{H}$ NMR spectrum of compound $4 \mathrm{ab}\left(400 \mathrm{MHz}, \mathrm{CDCl}_{3}\right)$

1fa760b-201902711
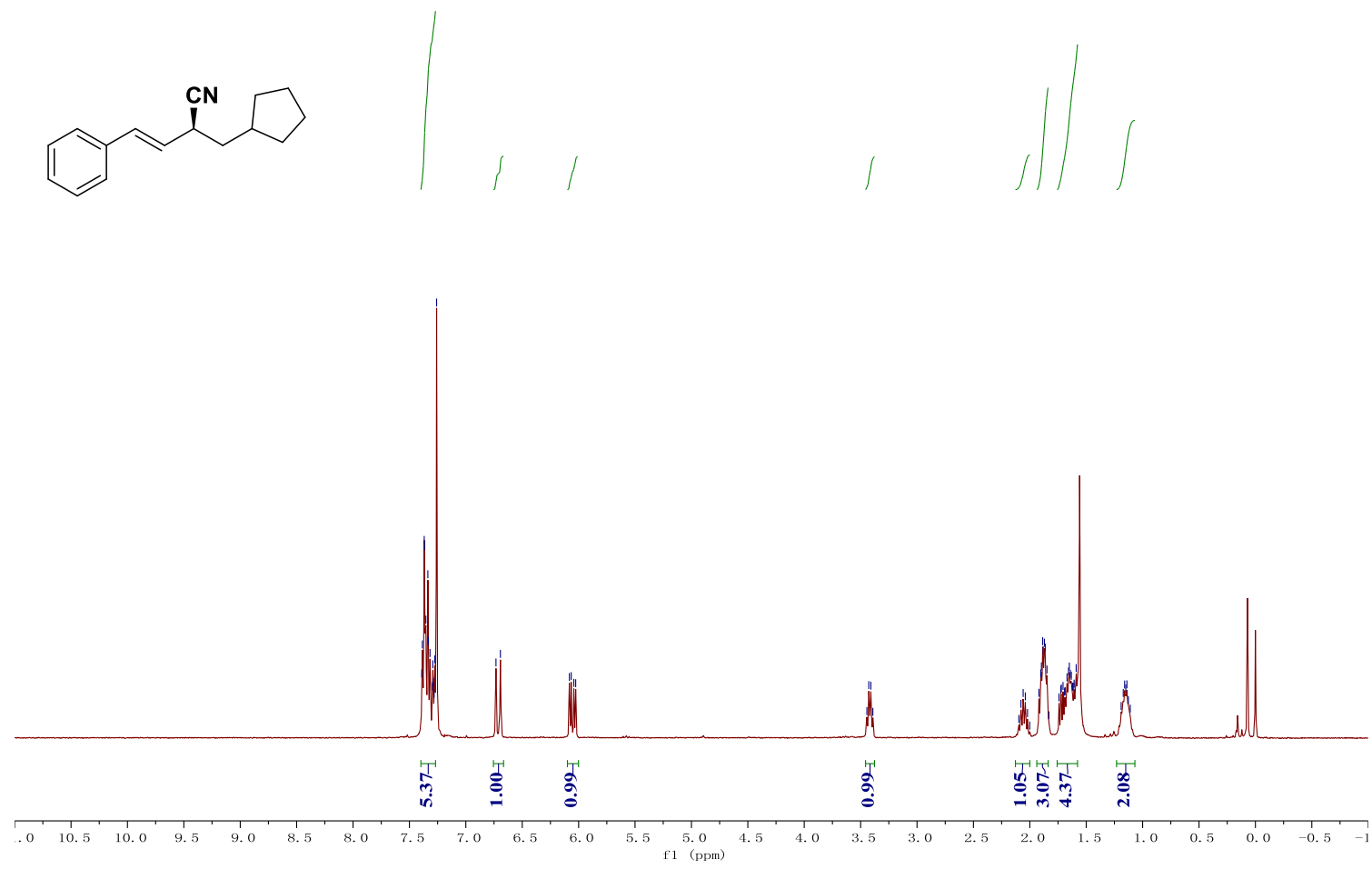

${ }^{13} \mathrm{C}$ NMR spectrum of compound $4 \mathrm{ab}\left(100 \mathrm{MHz}, \mathrm{CDCl}_{3}\right)$

1'd76062-20191027/2<smiles>N#CC(/C=C/c1ccccc1)CC1CCCC1</smiles>

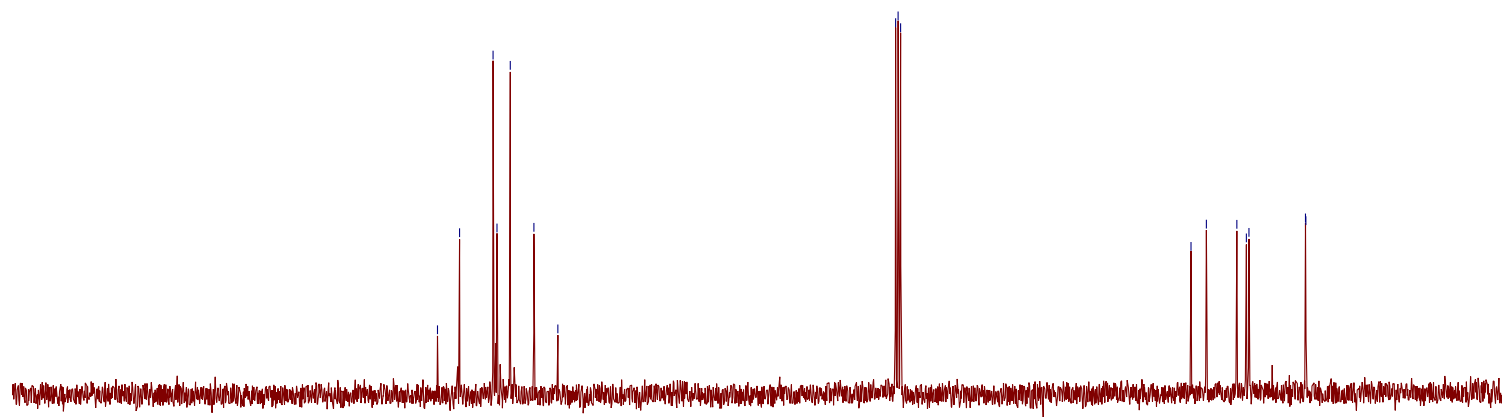

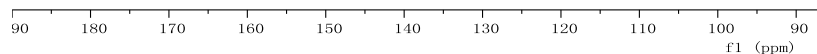


${ }^{1} \mathrm{H}$ NMR spectrum of compound $4 \mathrm{ac}\left(400 \mathrm{MHz}, \mathrm{CDCl}_{3}\right)$
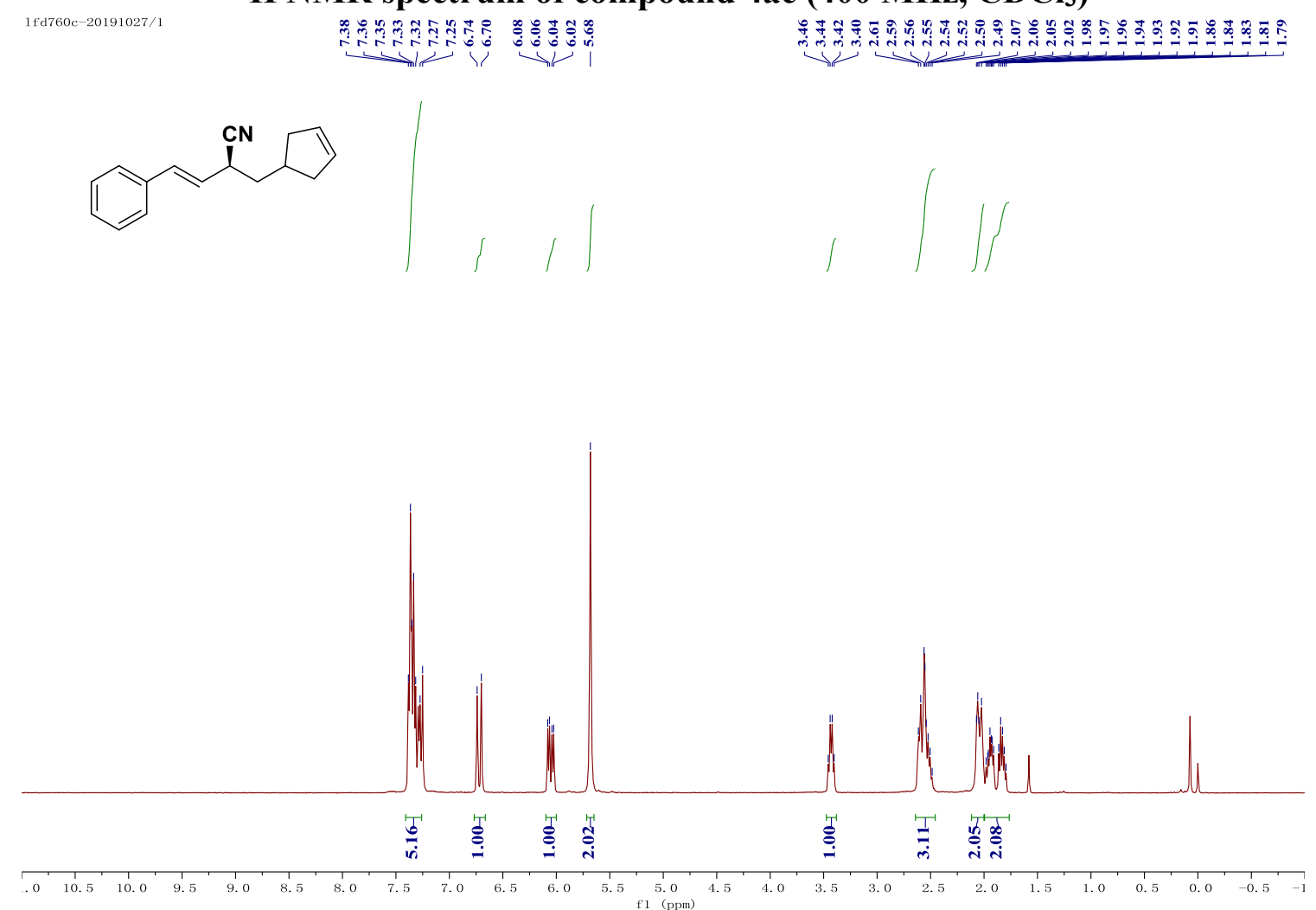

${ }^{13} \mathrm{C}$ NMR spectrum of compound $4 \mathrm{ac}\left(100 \mathrm{MHz}, \mathrm{CDCl}_{3}\right)$

1fd7600-20191027/2<smiles>N#CC(C=Cc1ccccc1)CC1CC=CC1</smiles>

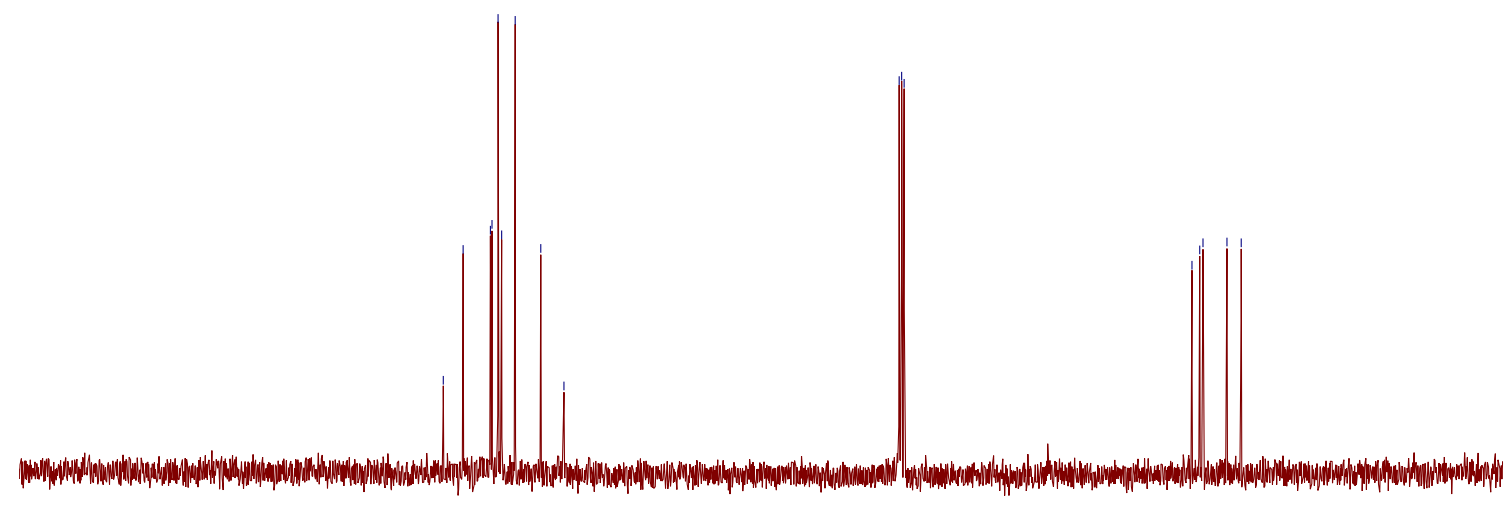


${ }^{1} \mathrm{H}$ NMR spectrum of compound $4 \mathrm{ad}\left(400 \mathrm{MHz}, \mathrm{CDCl}_{3}\right)$

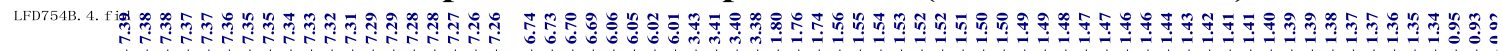<smiles>CCCC[C@H](C#N)/C=C/c1ccccc1</smiles><smiles>C1CCC1</smiles>

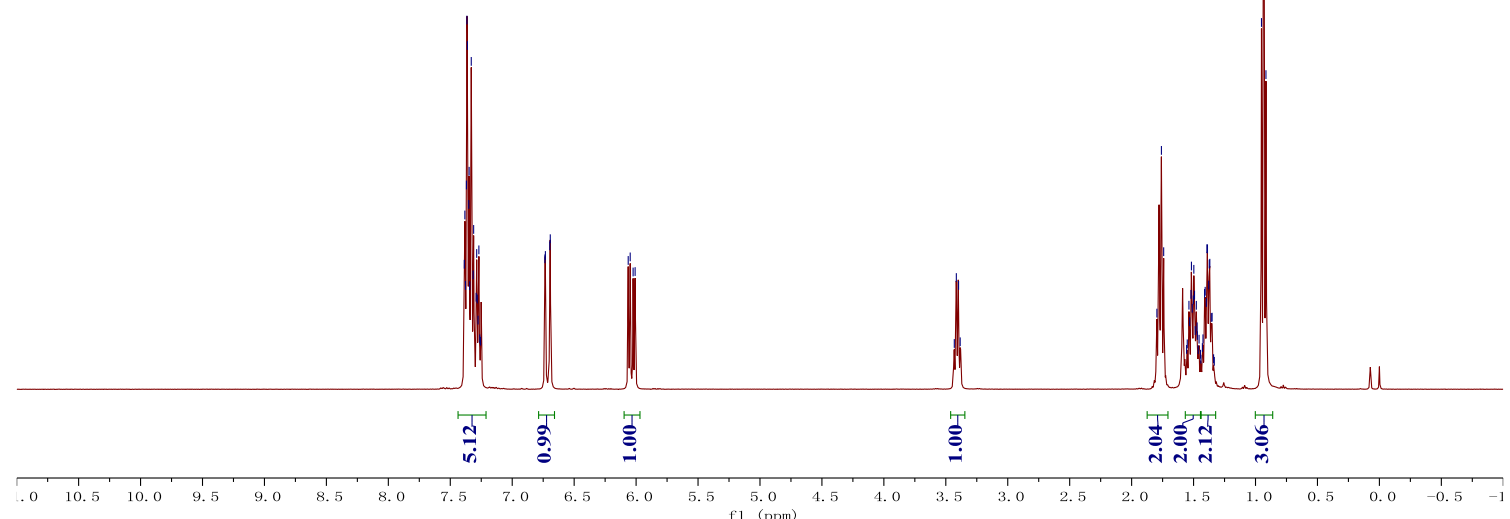

${ }^{13} \mathrm{C}$ NMR spectrum of compound $4 \mathrm{ad}\left(100 \mathrm{MHz}, \mathrm{CDCl}_{3}\right)$
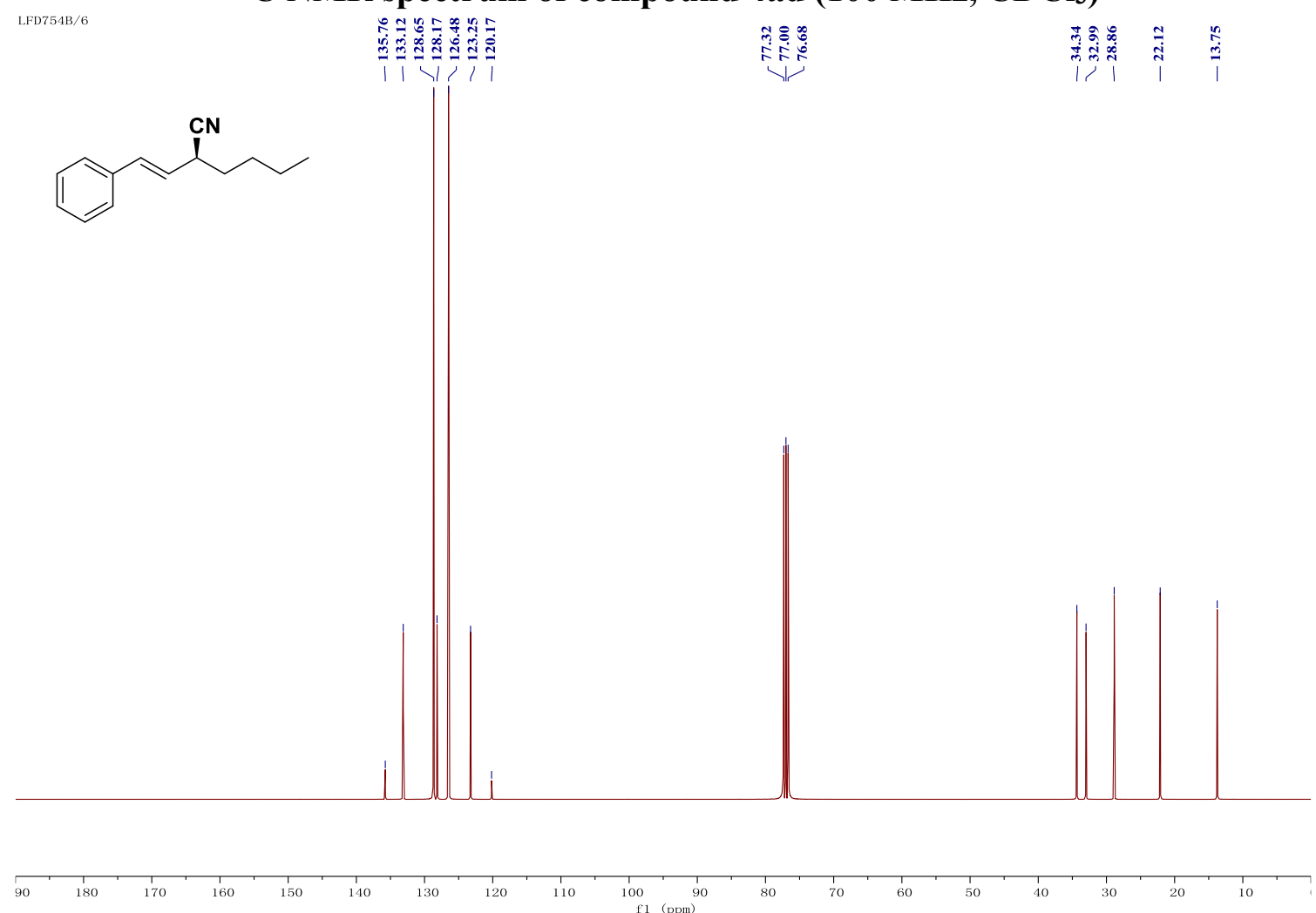
${ }^{1} \mathrm{H}$ NMR spectrum of compound $4 \mathrm{ae}\left(400 \mathrm{MHz}, \mathrm{CDCl}_{3}\right)$

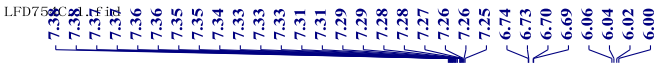

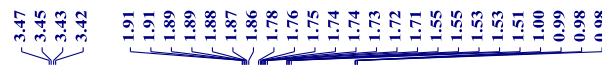
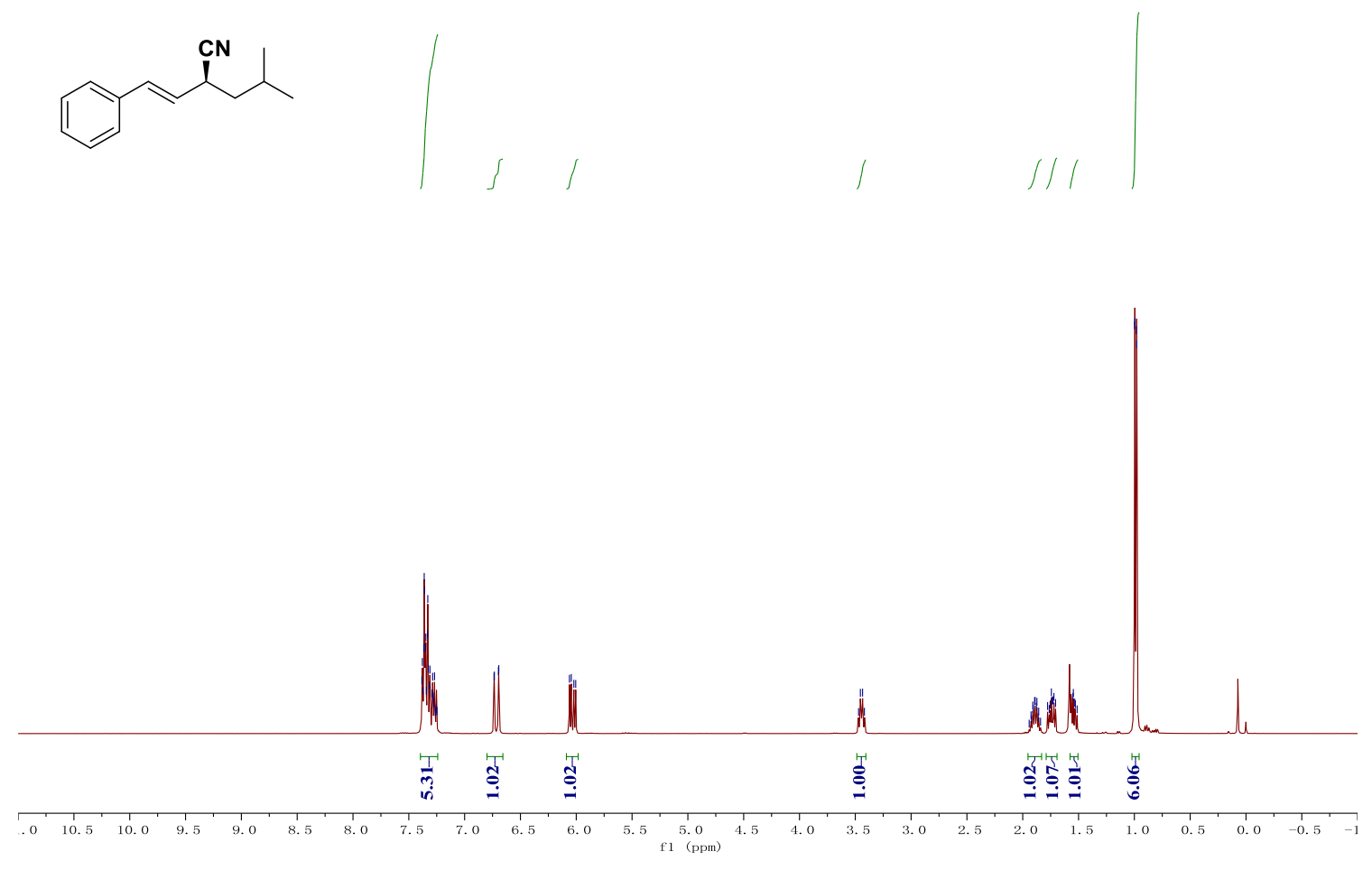

${ }^{13} \mathrm{C}$ NMR spectrum of compound $4 \mathrm{ae}\left(100 \mathrm{MHz}, \mathrm{CDCl}_{3}\right)$
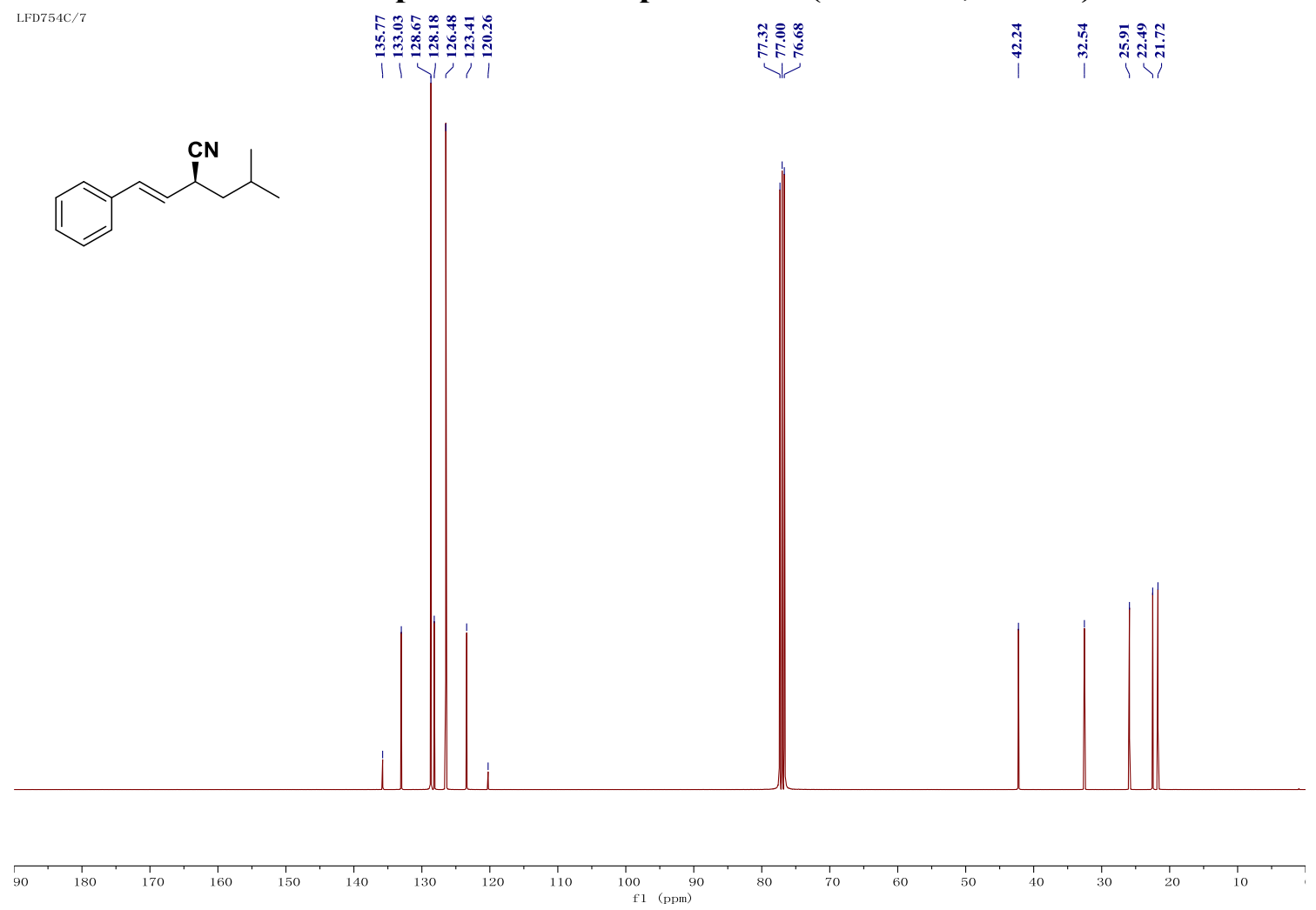

S37 
${ }^{1} \mathrm{H}$ NMR spectrum of compound 4 af $\left(400 \mathrm{MHz}, \mathrm{CDCl}_{3}\right)$

$1 \mathrm{fd} 760 \mathrm{a} / 1$

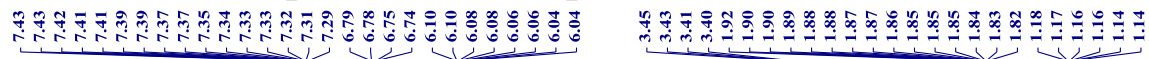
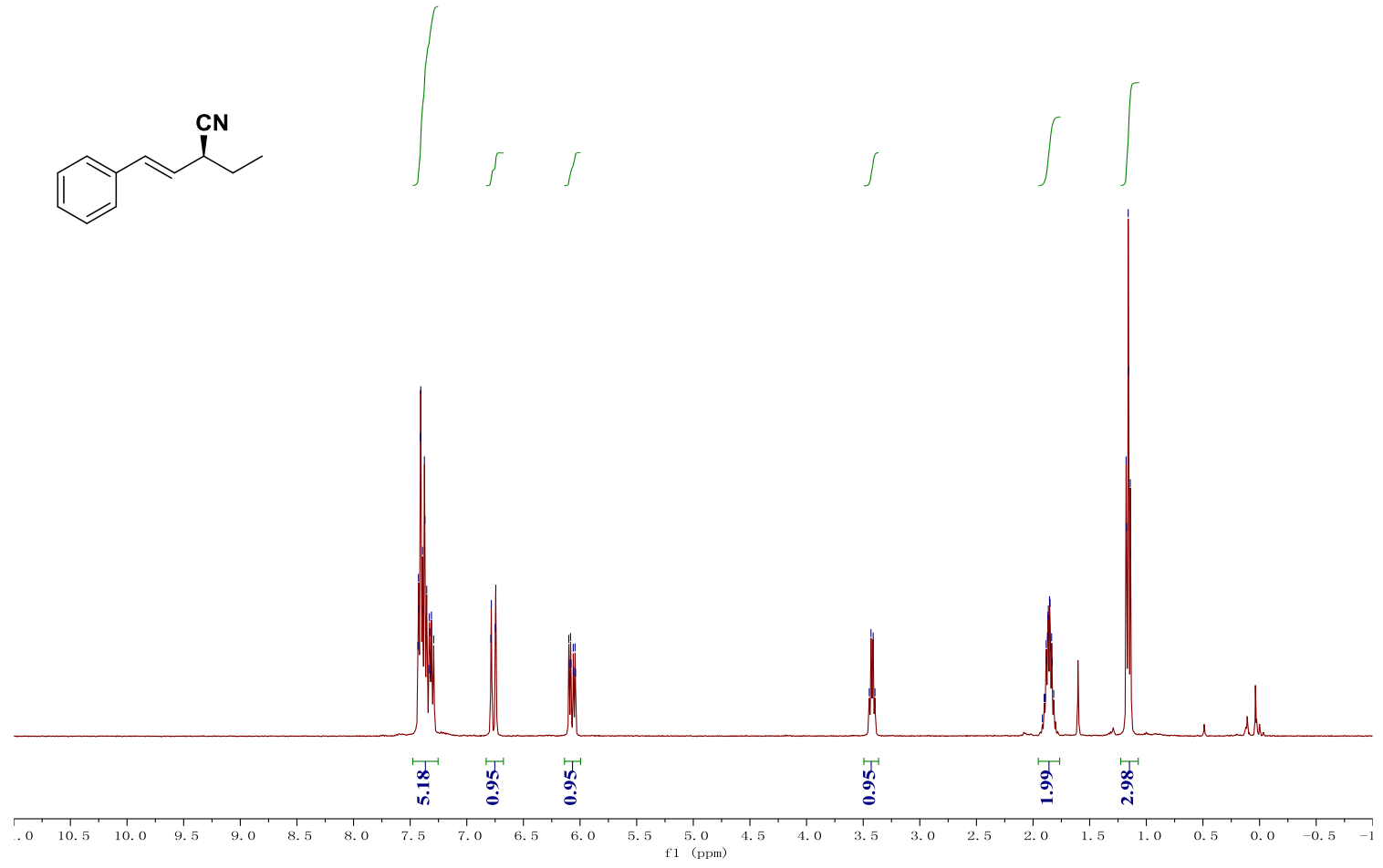

${ }^{13} \mathrm{C}$ NMR spectrum of compound 4 af $\left(100 \mathrm{MHz}, \mathrm{CDCl}_{3}\right)$

1fd760a - 20191027/2

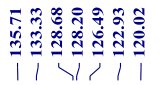

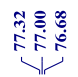

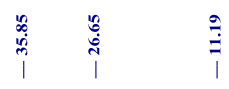
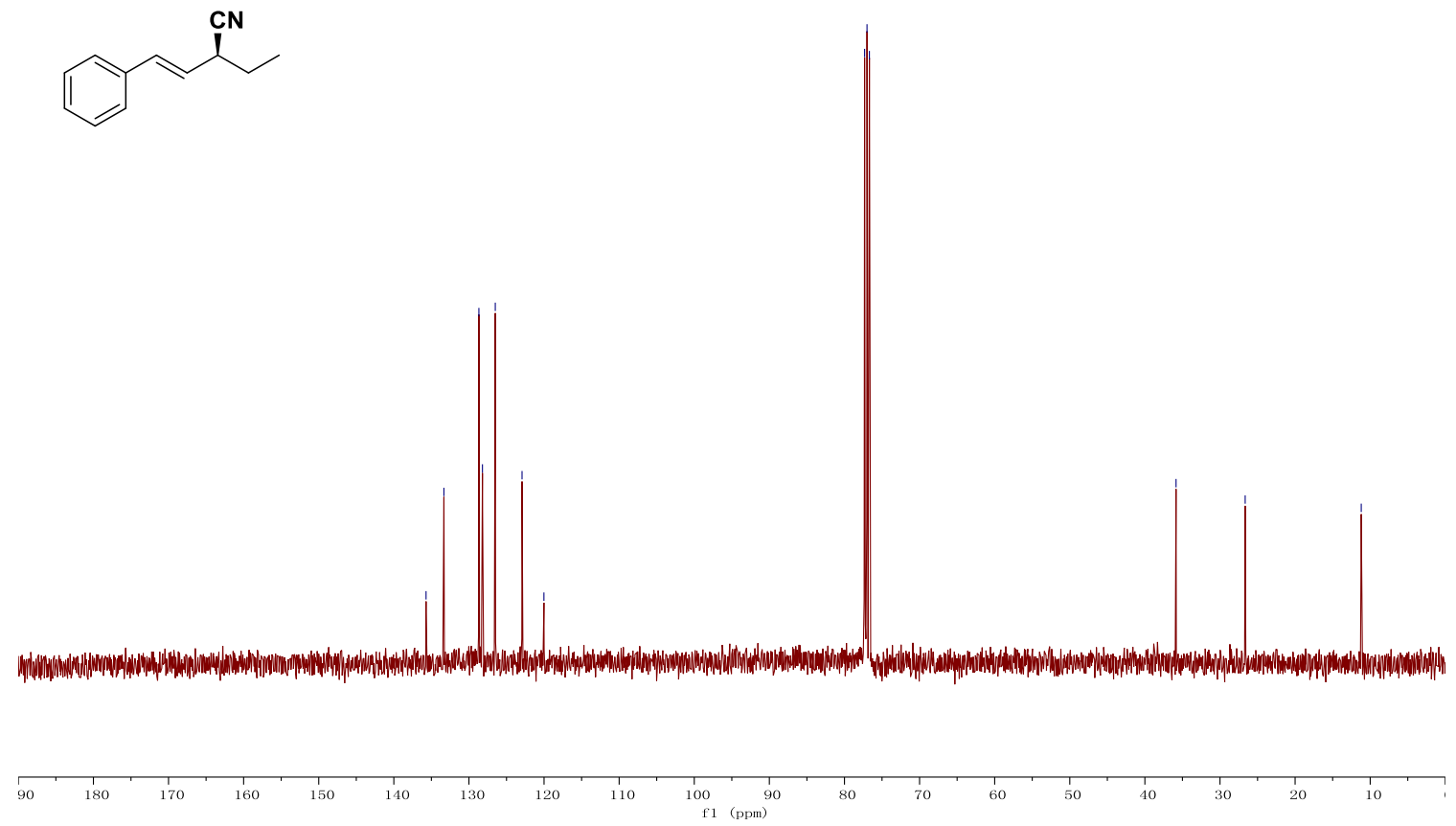

S38 
${ }^{1} \mathrm{H}$ NMR spectrum of compound $4 \mathrm{ag}\left(400 \mathrm{MHz}, \mathrm{CDCl}_{3}\right)$

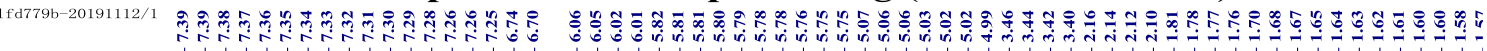

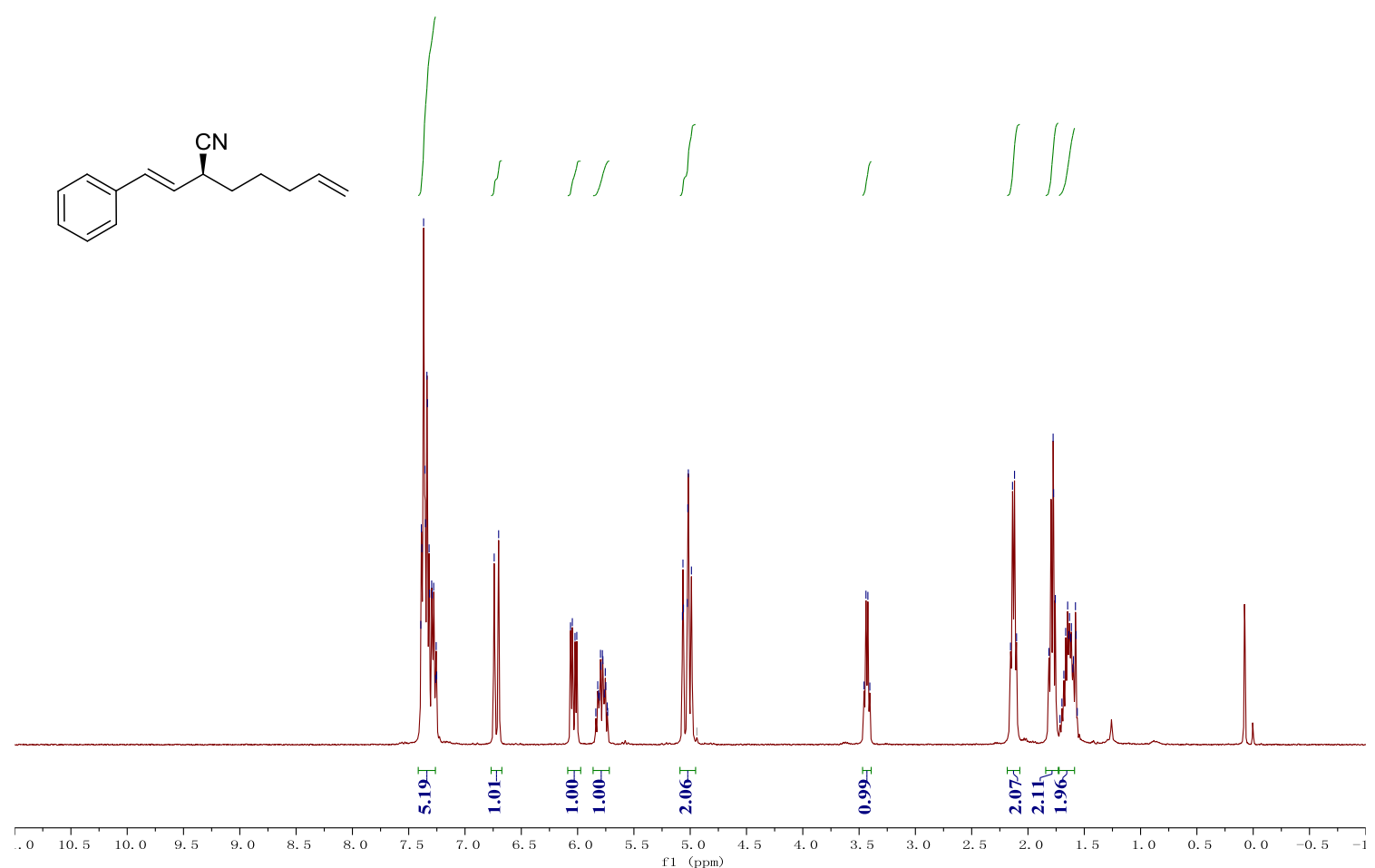

${ }^{13} \mathrm{C}$ NMR spectrum of compound $4 \mathrm{ag}\left(100 \mathrm{MHz}, \mathrm{CDCl}_{3}\right)$

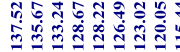

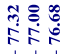

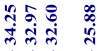
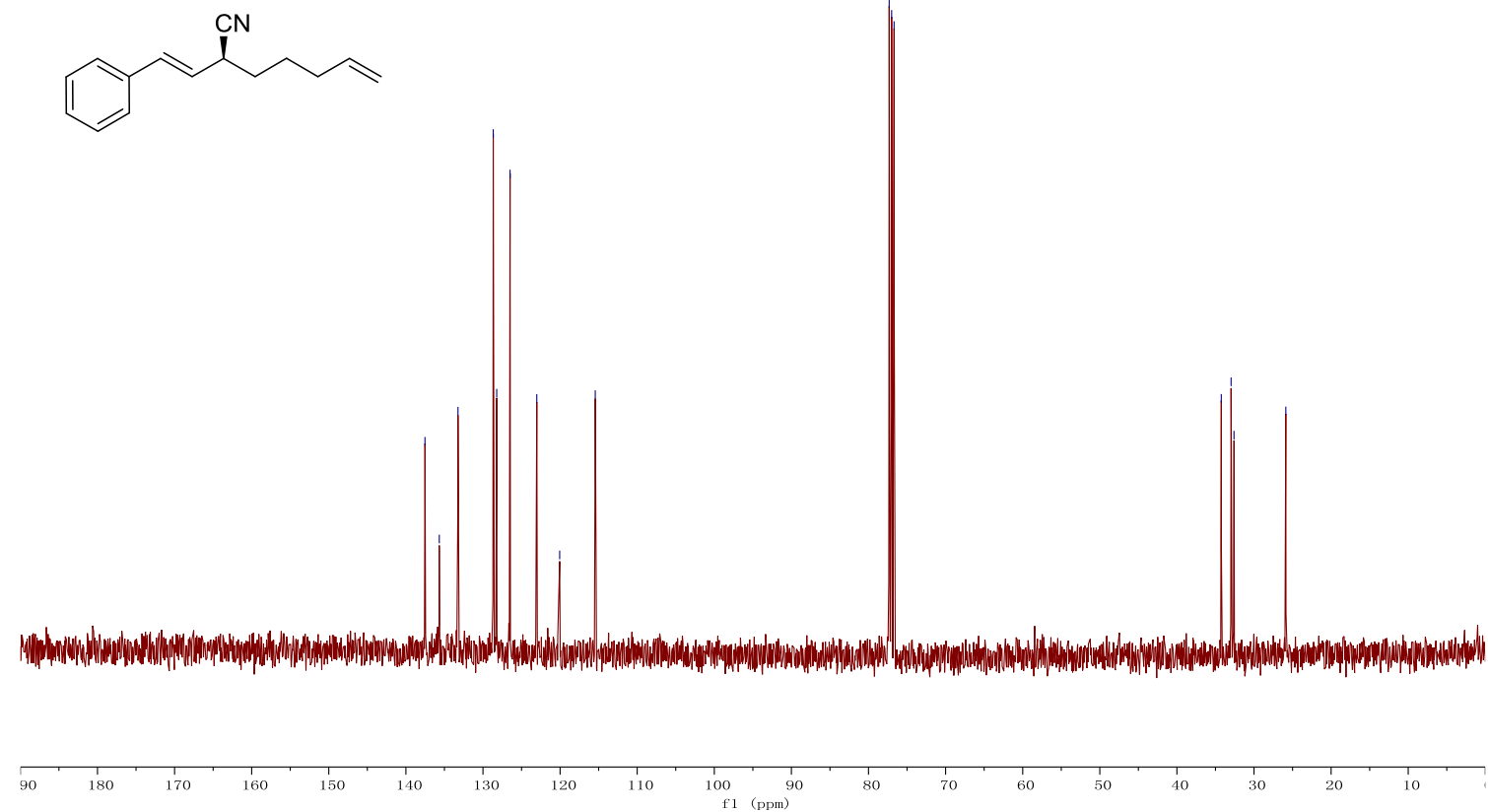
${ }^{1} \mathrm{H}$ NMR spectrum of compound $4 \mathrm{ah}\left(400 \mathrm{MHz}, \mathrm{CDCl}_{3}\right)$

1fd779c-20191112/2

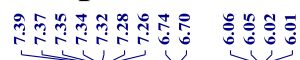

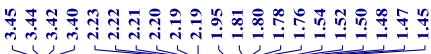<smiles>C#CC(C#N)/C=C/c1ccccc1</smiles><smiles>C1CCCC1</smiles>
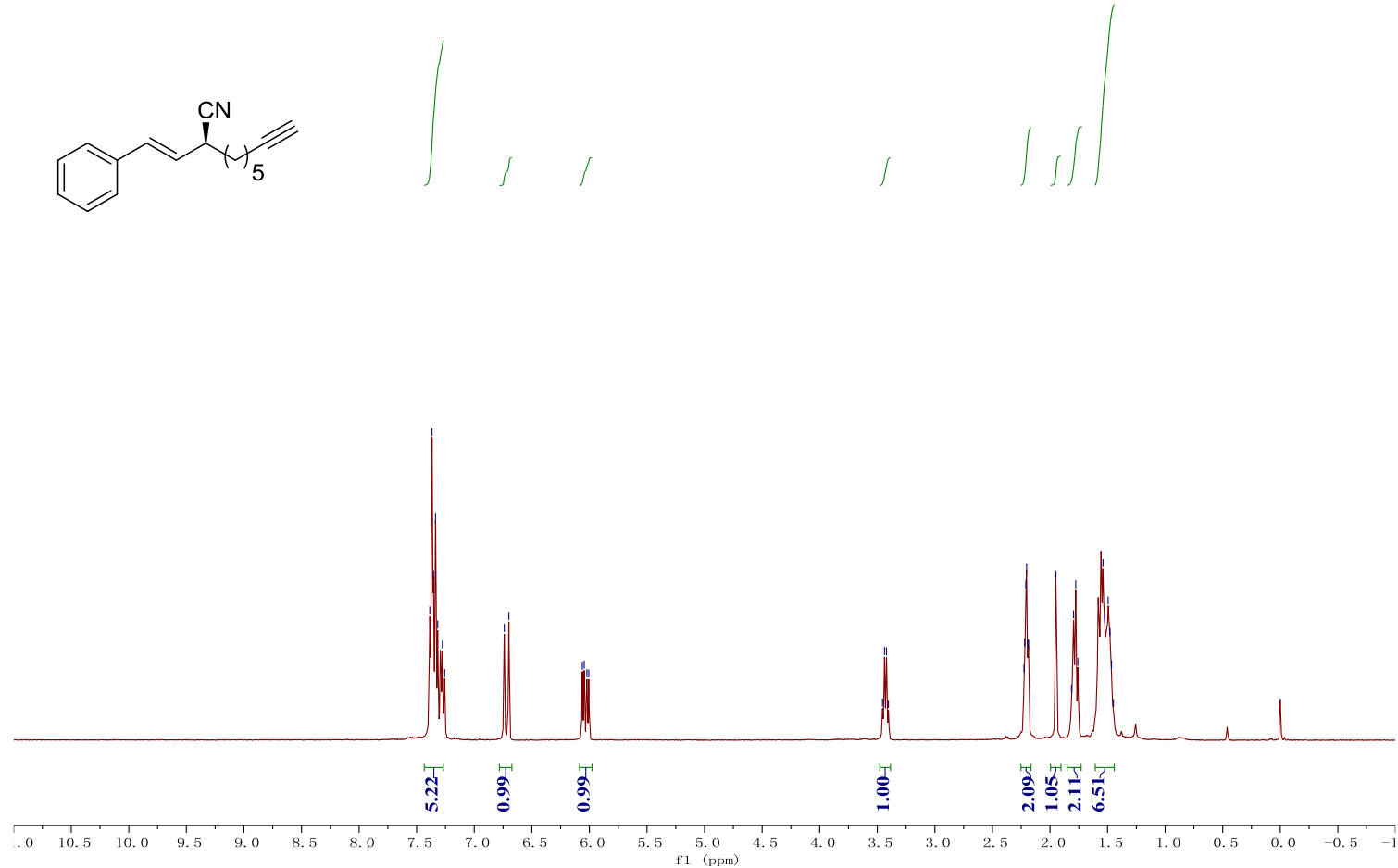

${ }^{13} \mathrm{C}$ NMR spectrum of compound $4 \mathrm{ah}\left(100 \mathrm{MHz}, \mathrm{CDCl}_{3}\right)$<smiles>C#CC(C#N)C=Cc1ccccc1</smiles>

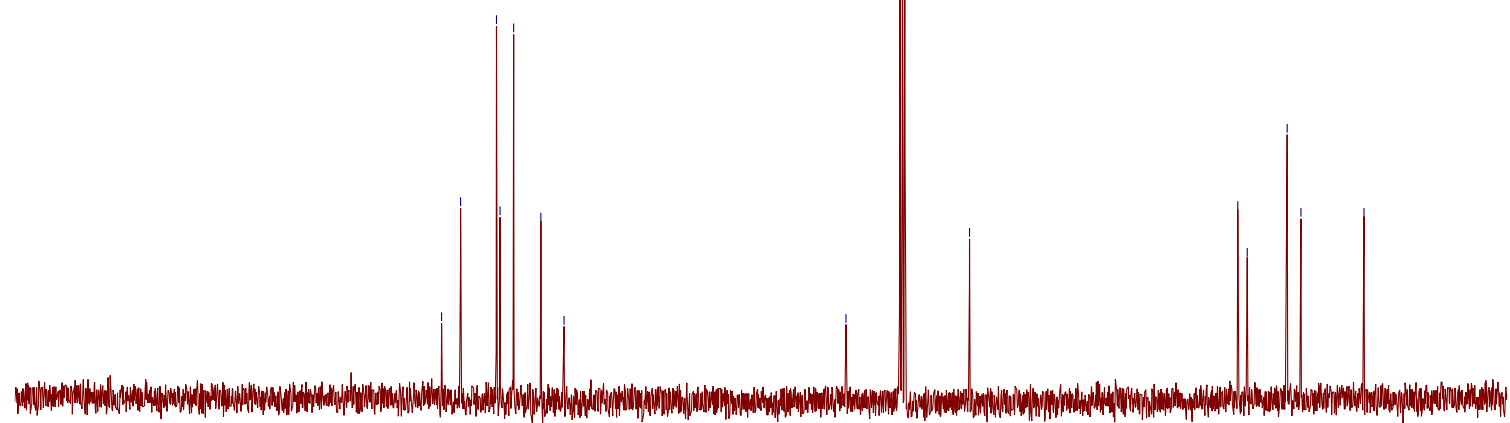

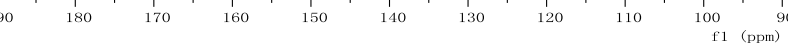


${ }^{1} \mathrm{H}$ NMR spectrum of compound $4 \mathrm{ai}\left(400 \mathrm{MHz}, \mathrm{CDCl}_{3}\right)$<smiles>N#CC(/C=C/c1ccccc1)CCCCCl</smiles>
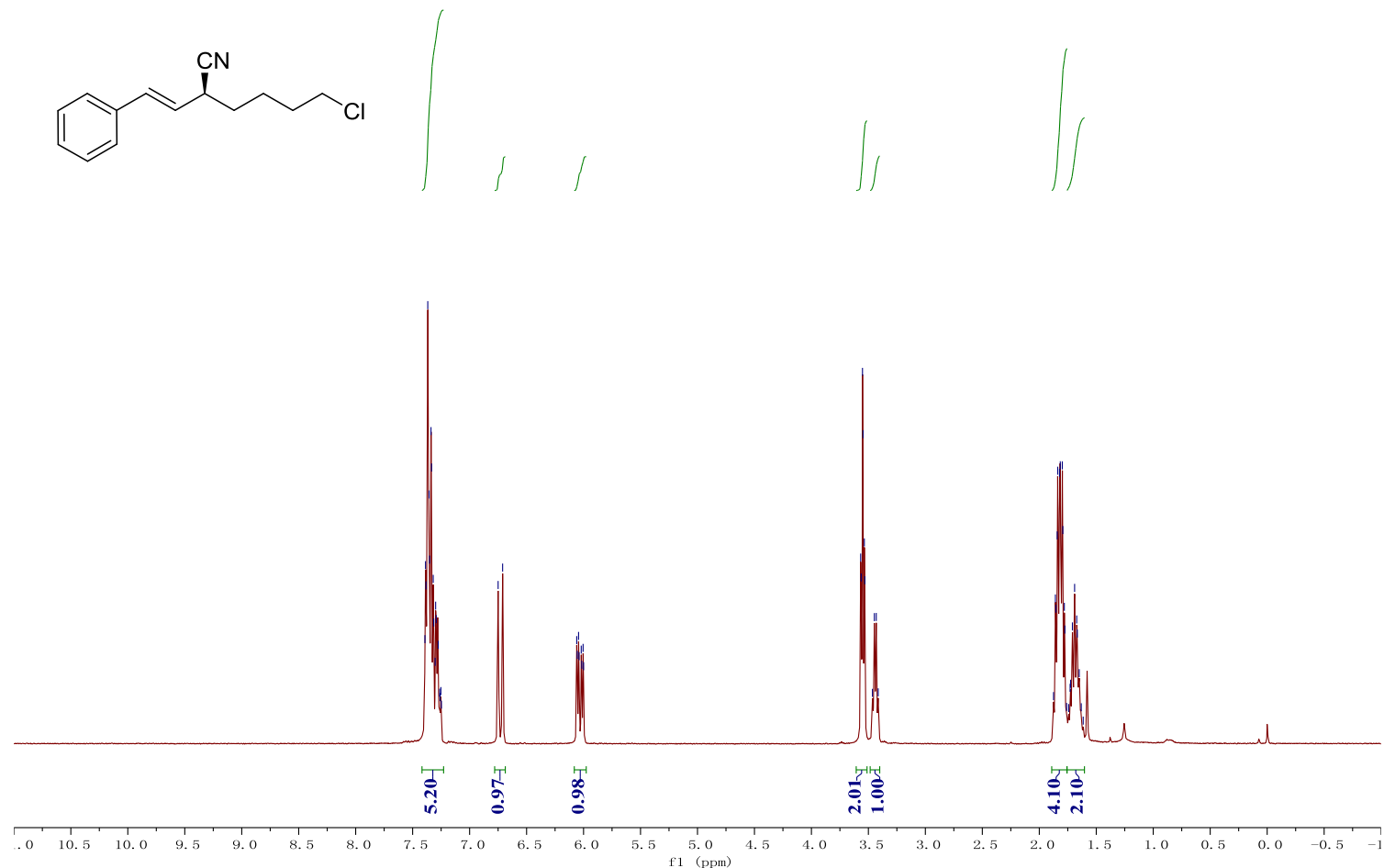

${ }^{13} \mathrm{C}$ NMR spectrum of compound 4ai $\left(100 \mathrm{MHz}, \mathrm{CDCl}_{3}\right)$<smiles>N#C[C@H](/C=C/c1ccccc1)CCCCCl</smiles>

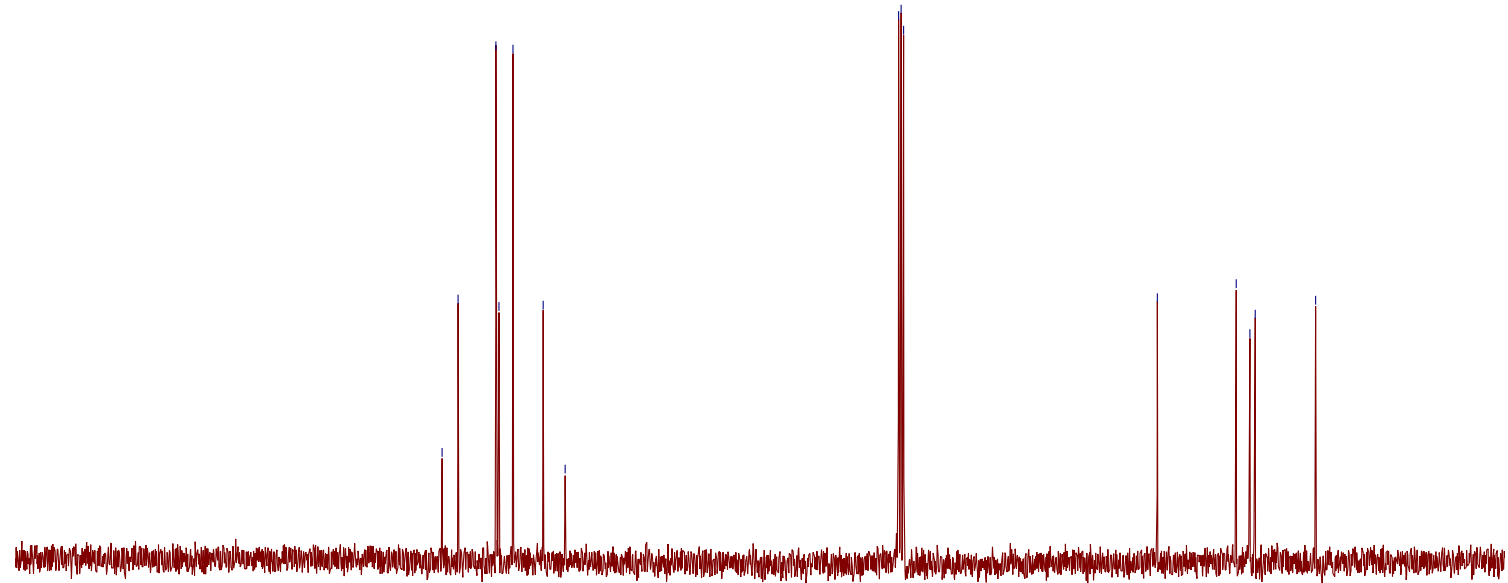

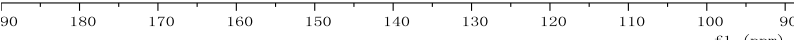


${ }^{1} \mathrm{H}$ NMR spectrum of compound $4 \mathrm{bd}\left(400 \mathrm{MHz}, \mathrm{CDCl}_{3}\right)$

$1 \mathrm{fd} 796 \mathrm{a}-2-20191204 / 1$

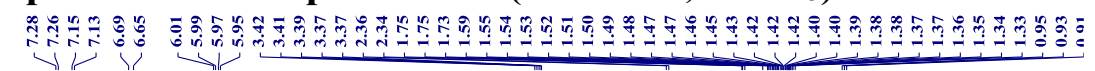
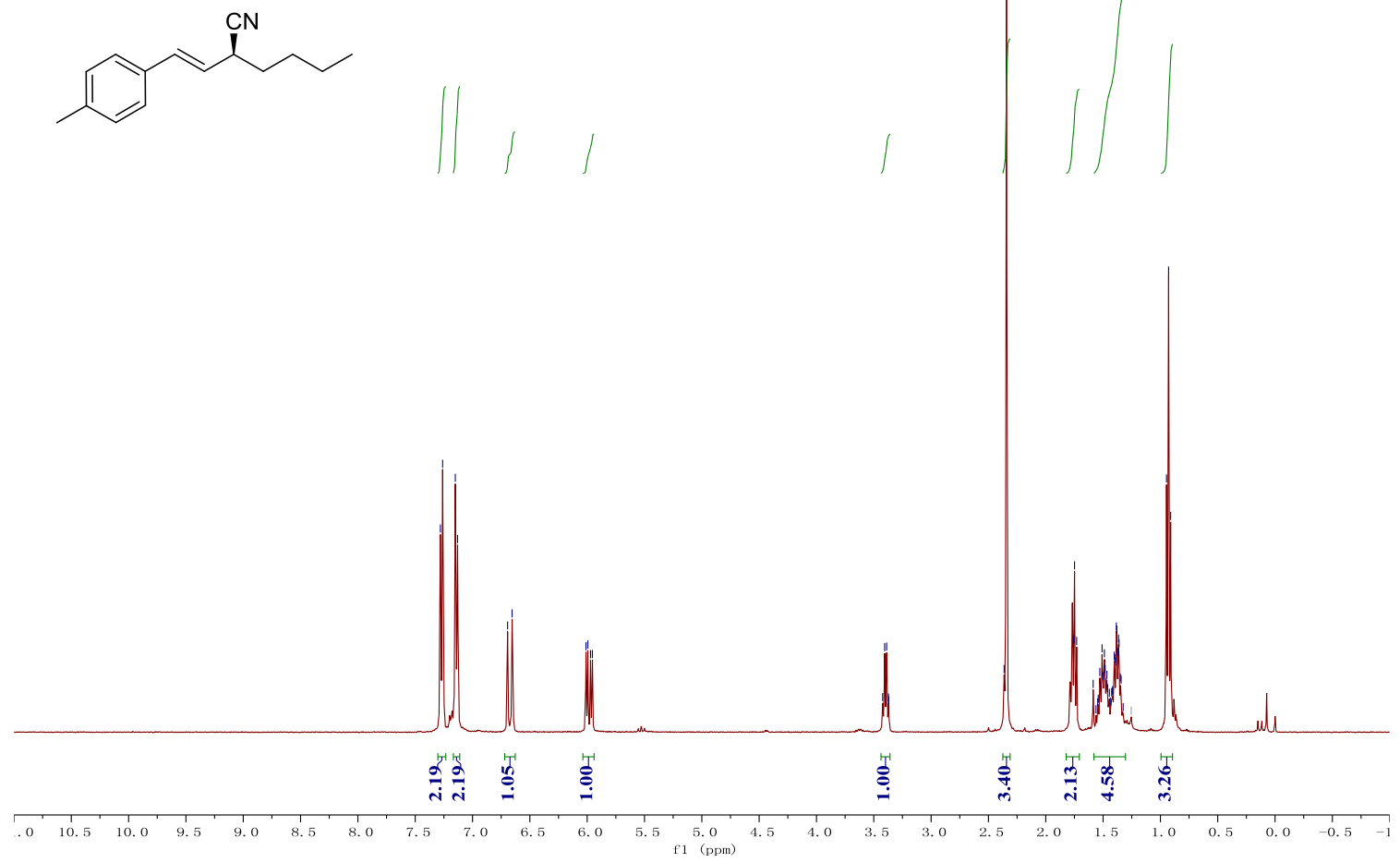

${ }^{13} \mathrm{C}$ NMR spectrum of compound $4 \mathrm{bd}\left(100 \mathrm{MHz}, \mathrm{CDCl}_{3}\right)$

1fd796a-2-20191204/2

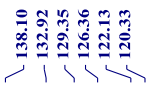

فำ

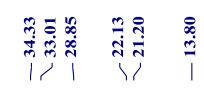
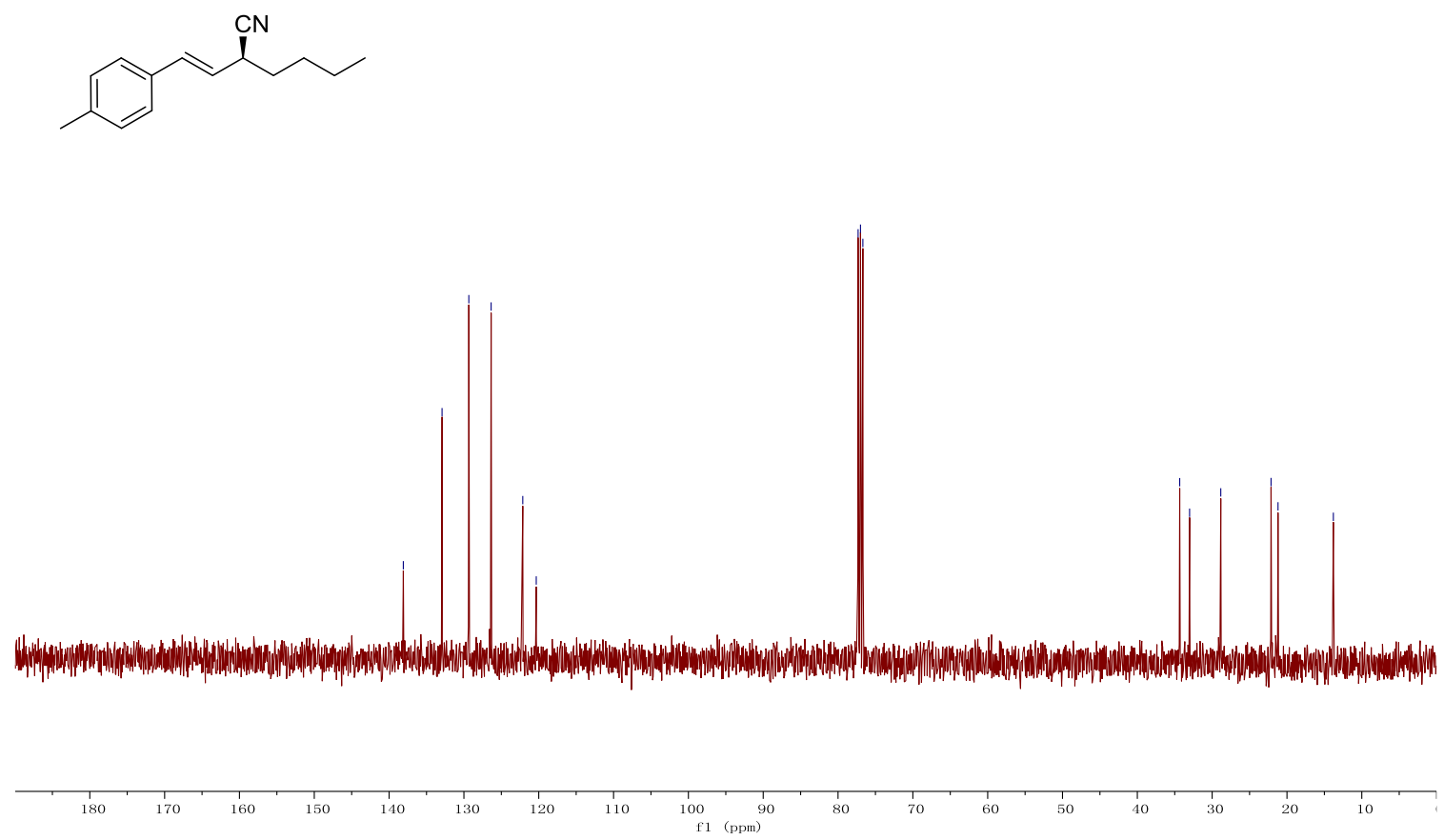

S42 
${ }^{1} \mathrm{H}$ NMR spectrum of compound $4 \mathrm{~cd}\left(400 \mathrm{MHz}, \mathrm{CDCl}_{3}\right)$

$1 \mathrm{fd} 796 \mathrm{~b} / 1$

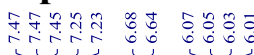

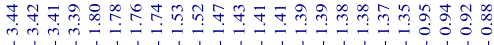<smiles>CCCCC(C#N)/C=C/c1ccc(Br)cc1</smiles>
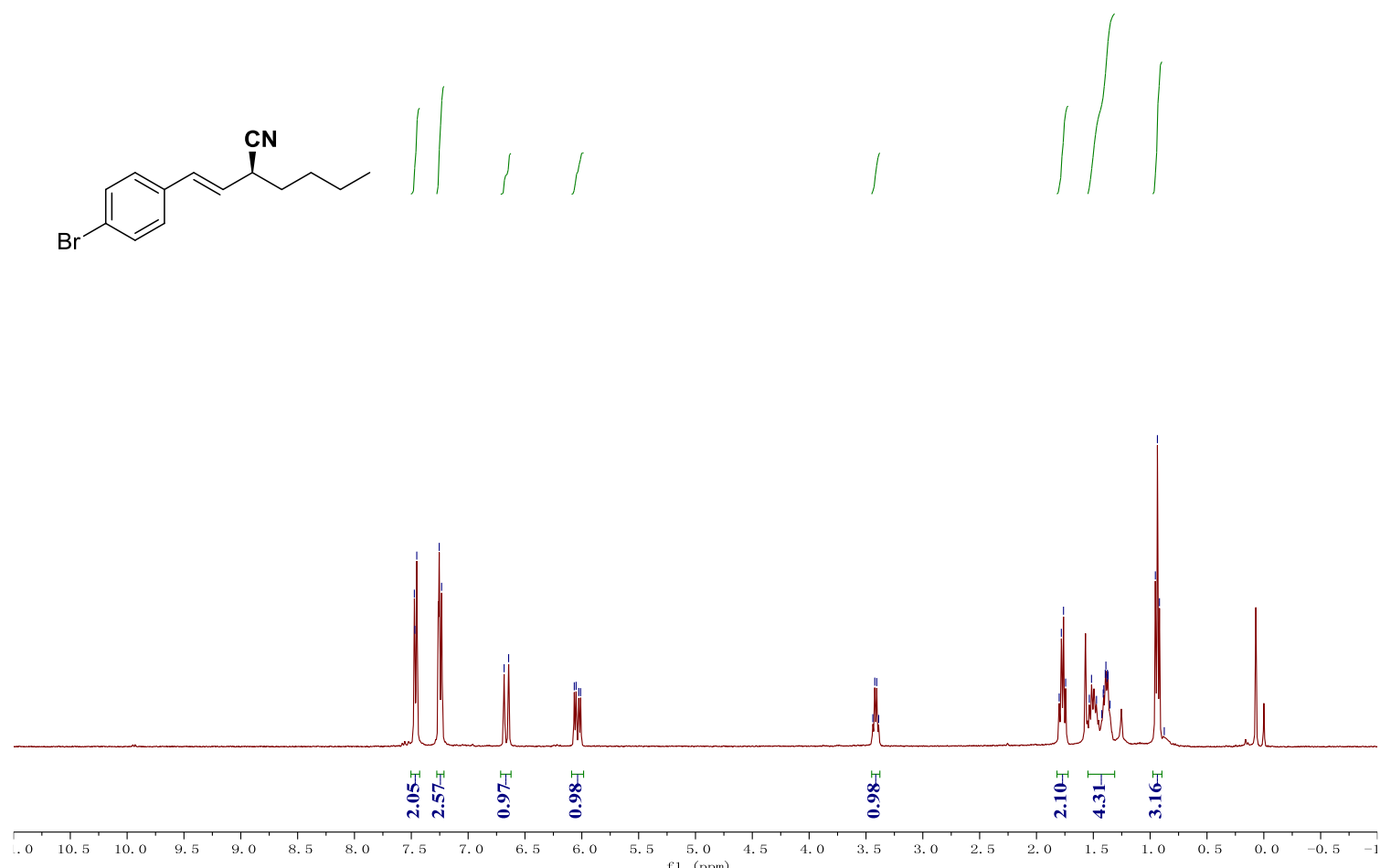

${ }^{13} \mathrm{C}$ NMR spectrum of compound $4 \mathrm{~cd}\left(100 \mathrm{MHz}, \mathrm{CDCl}_{3}\right)$

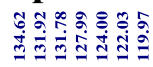

$1 \vee, 1+1$

要<smiles>CCCCC(C#N)/C=C/c1ccc(Br)cc1</smiles>

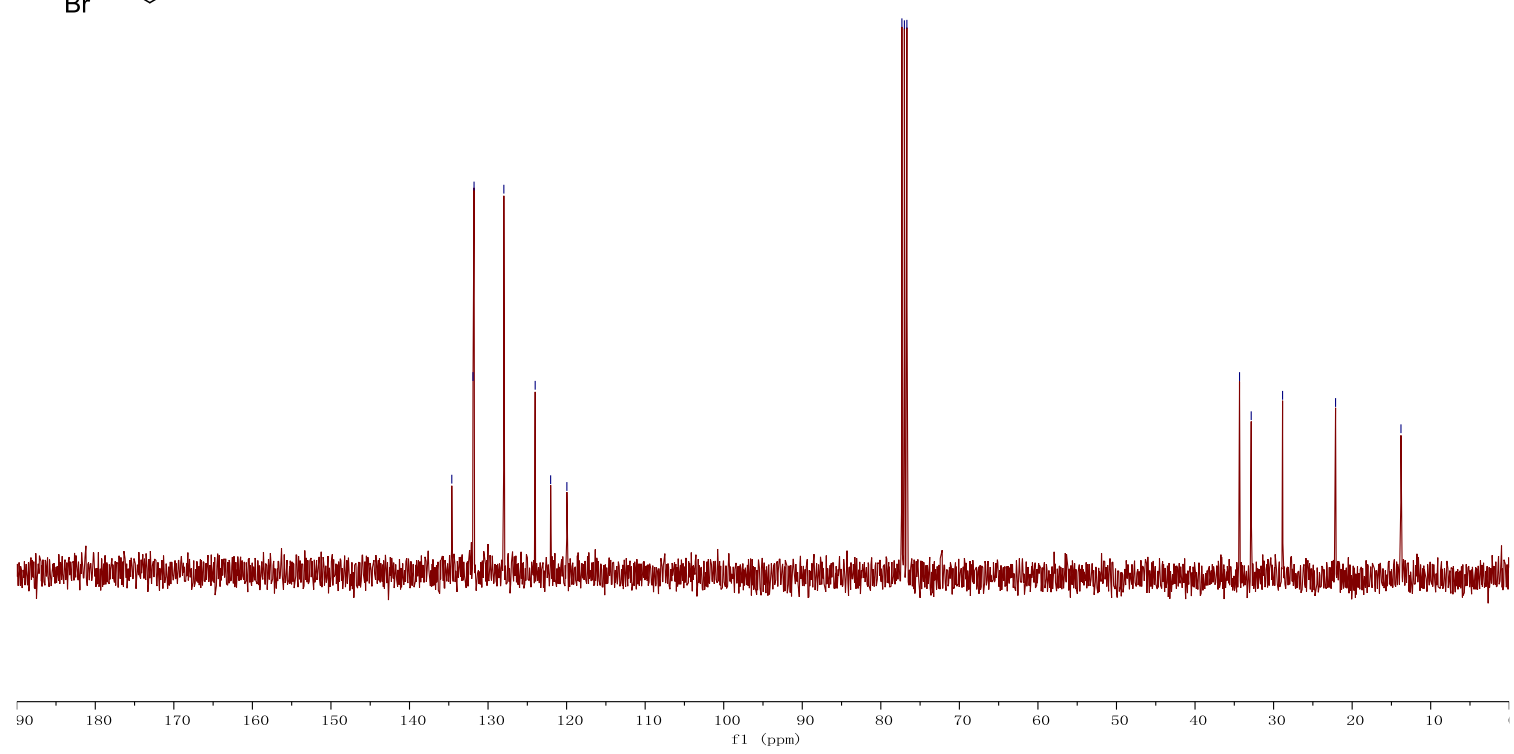


${ }^{1} \mathrm{H}$ NMR spectrum of compound $4 \mathrm{dd}\left(400 \mathrm{MHz}, \mathrm{CDCl}_{3}\right)$

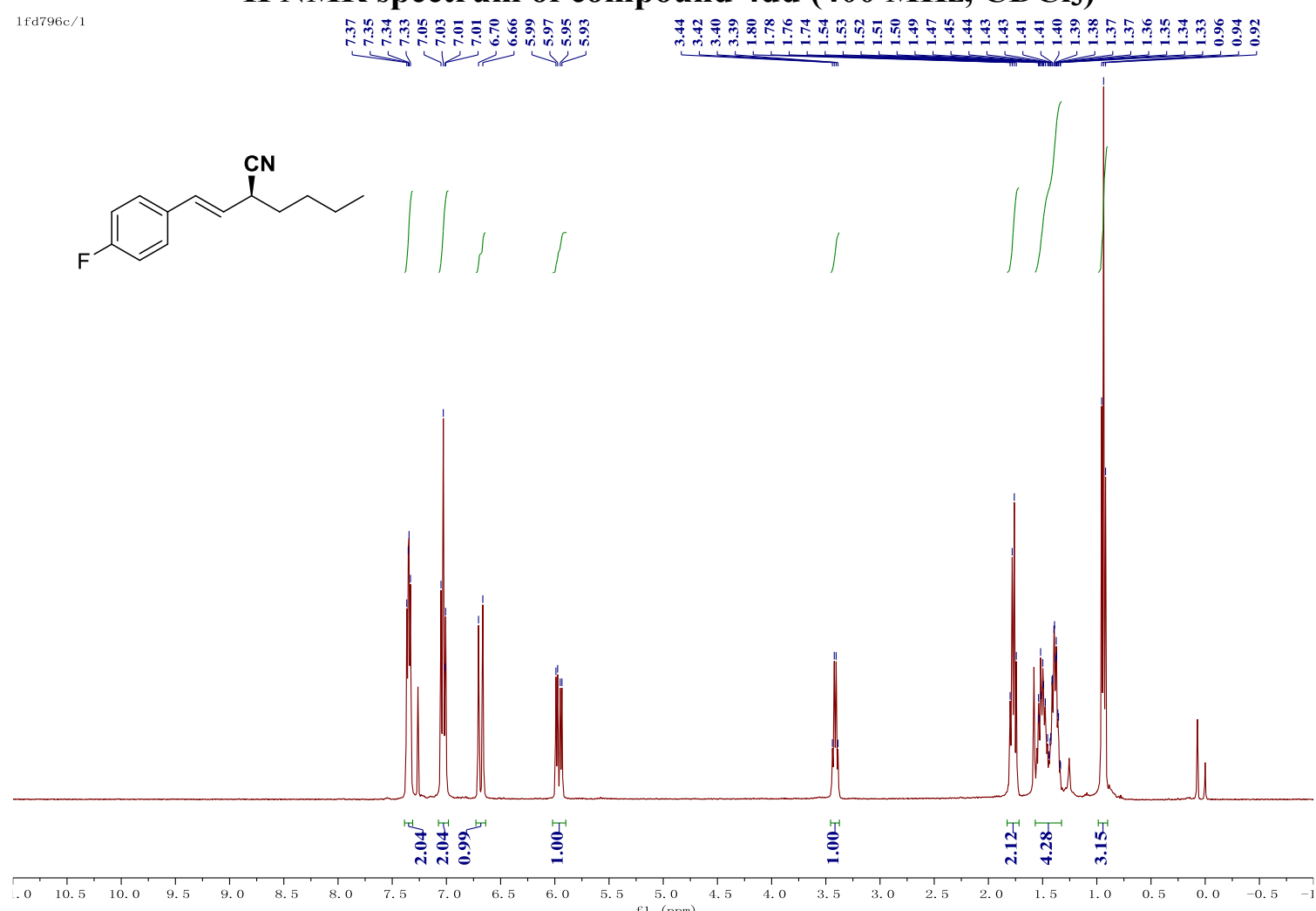

${ }^{13} \mathrm{C}$ NMR spectrum of compound $4 \mathrm{dd}\left(100 \mathrm{MHz}, \mathrm{CDCl}_{3}\right)$

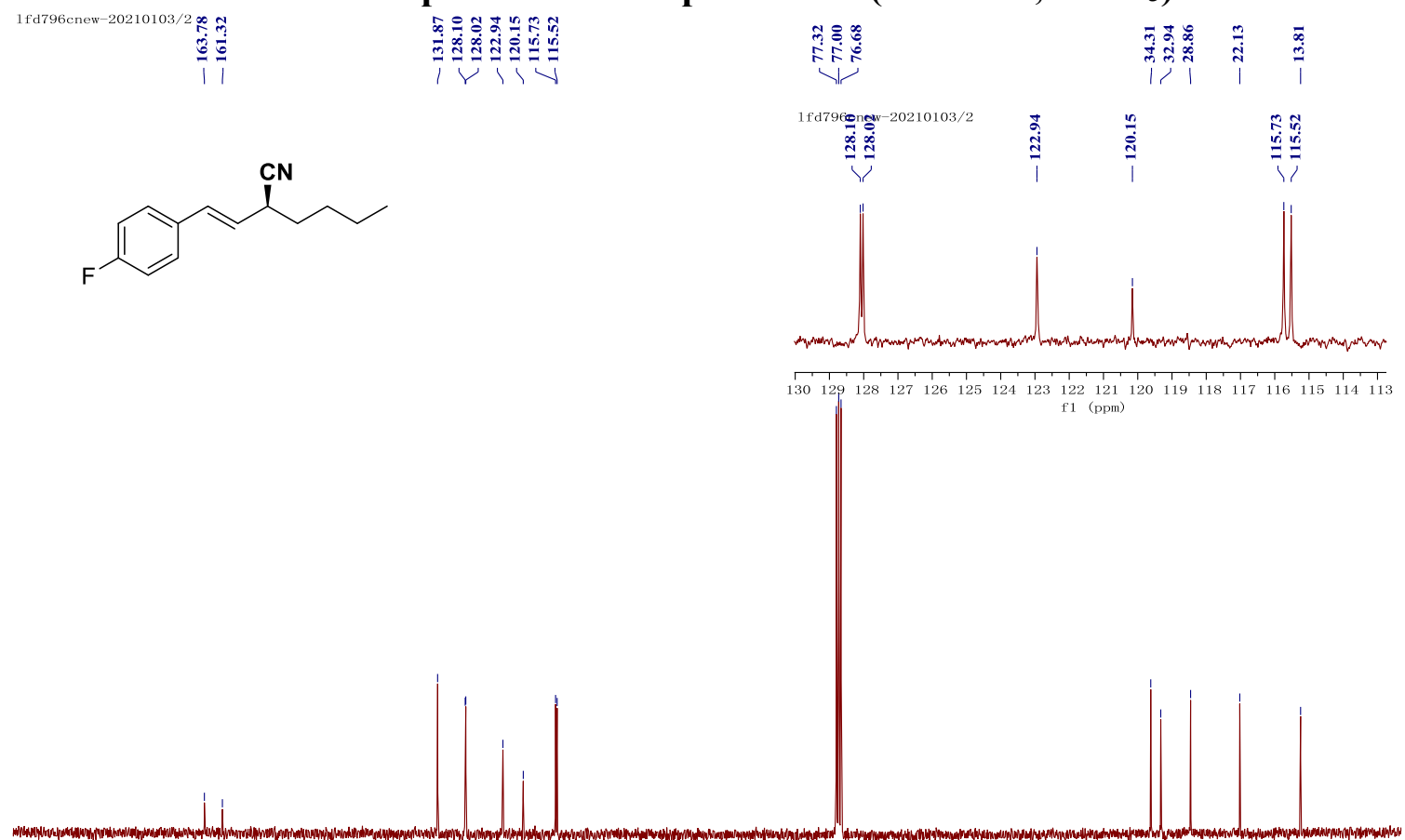

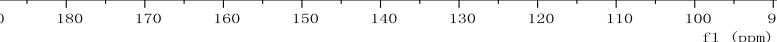


${ }^{19}$ F NMR spectrum of compound $4 \mathrm{dd}\left(376 \mathrm{MHz} \mathrm{CDCl}_{3}\right)$

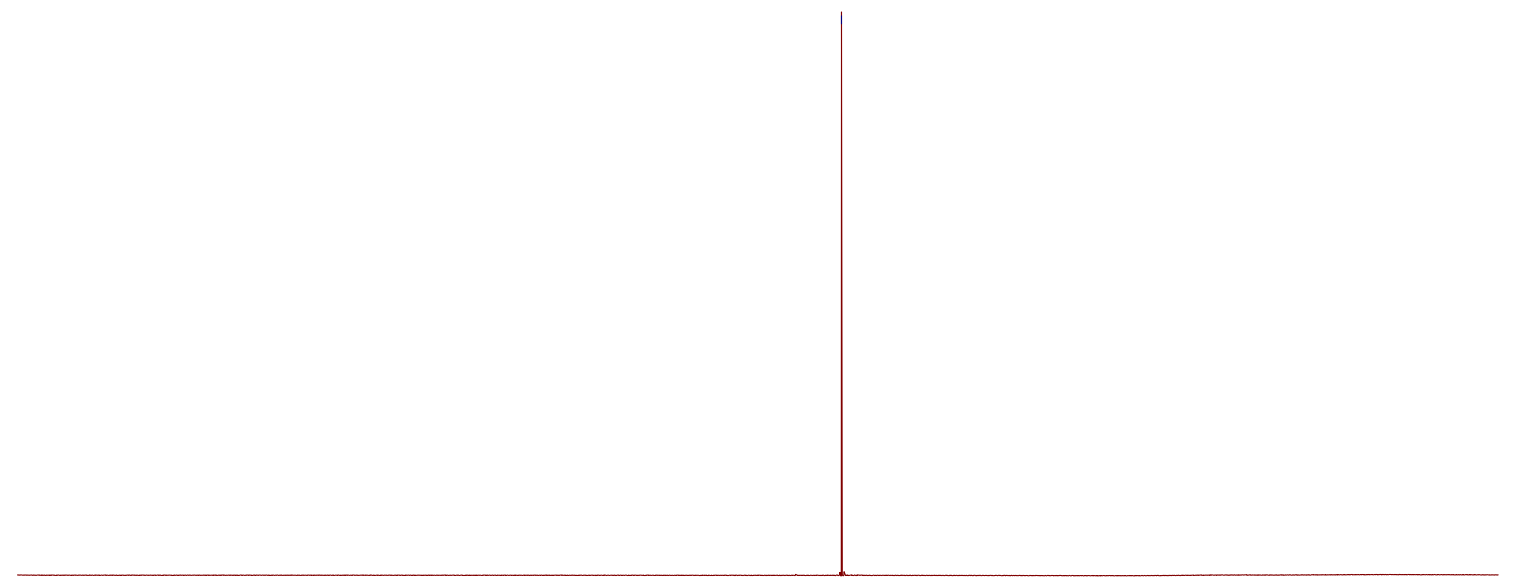

${ }^{1} \mathrm{H}$ NMR spectrum of compound $4 \mathrm{ed}\left(400 \mathrm{MHz}, \mathrm{CDCl}_{3}\right)$

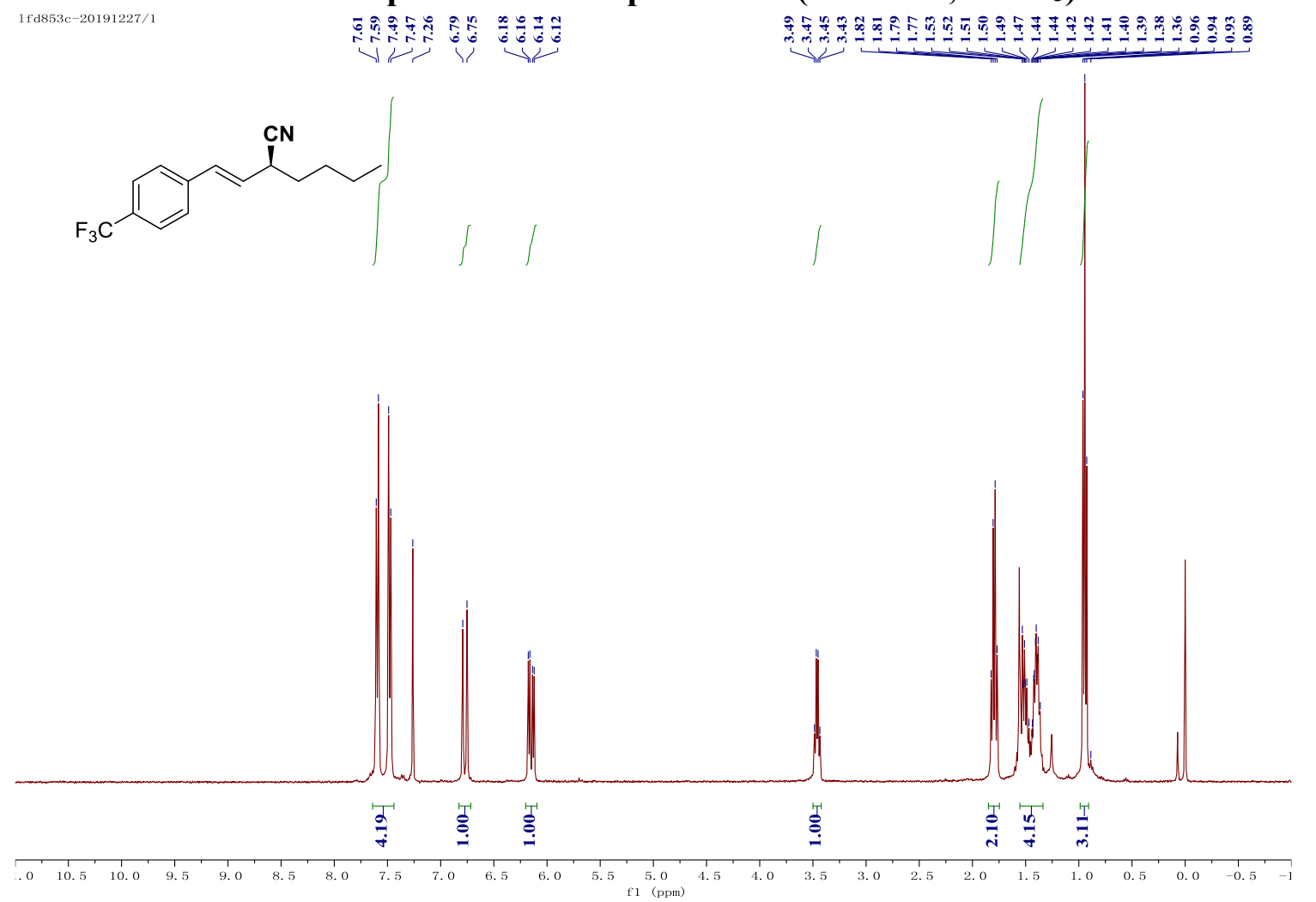


${ }^{13} \mathrm{C}$ NMR spectrum of compound $4 \mathrm{ed}\left(100 \mathrm{MHz}, \mathrm{CDCl}_{3}\right)$

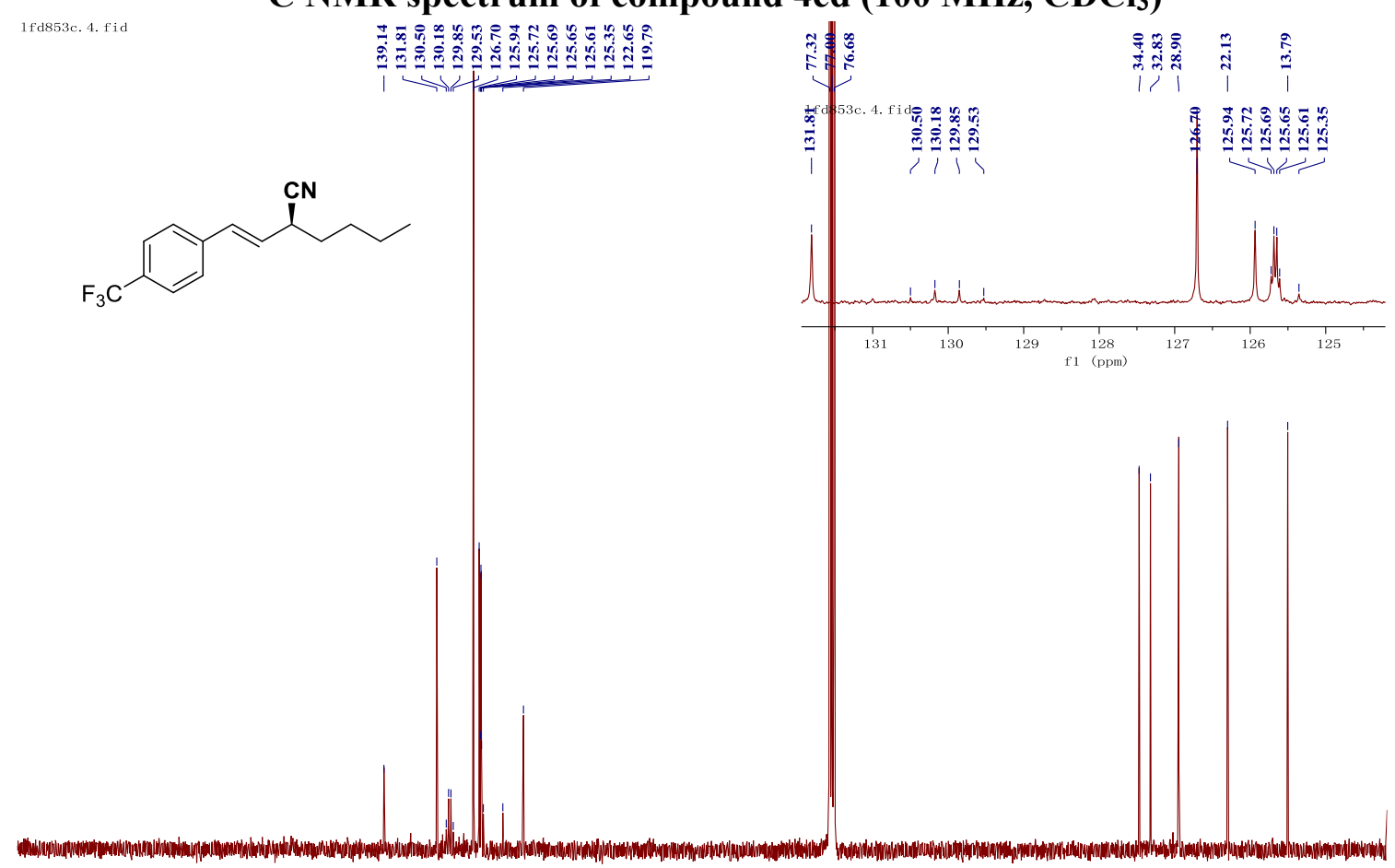

${ }^{19} \mathrm{~F}$ NMR spectrum of compound $4 \mathrm{ed}\left(376 \mathrm{MHz}, \mathrm{CDCl}_{3}\right)$<smiles>CCCCC(C#N)/C=C/c1ccc(C(F)(F)F)cc1</smiles> 
${ }^{1} \mathrm{H}$ NMR spectrum of compound $4 \mathrm{fd}\left(400 \mathrm{MHz}, \mathrm{CDCl}_{3}\right)$

$1 \mathrm{fd} 788 \mathrm{c} / 1$

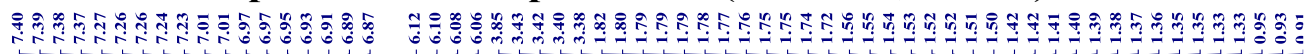
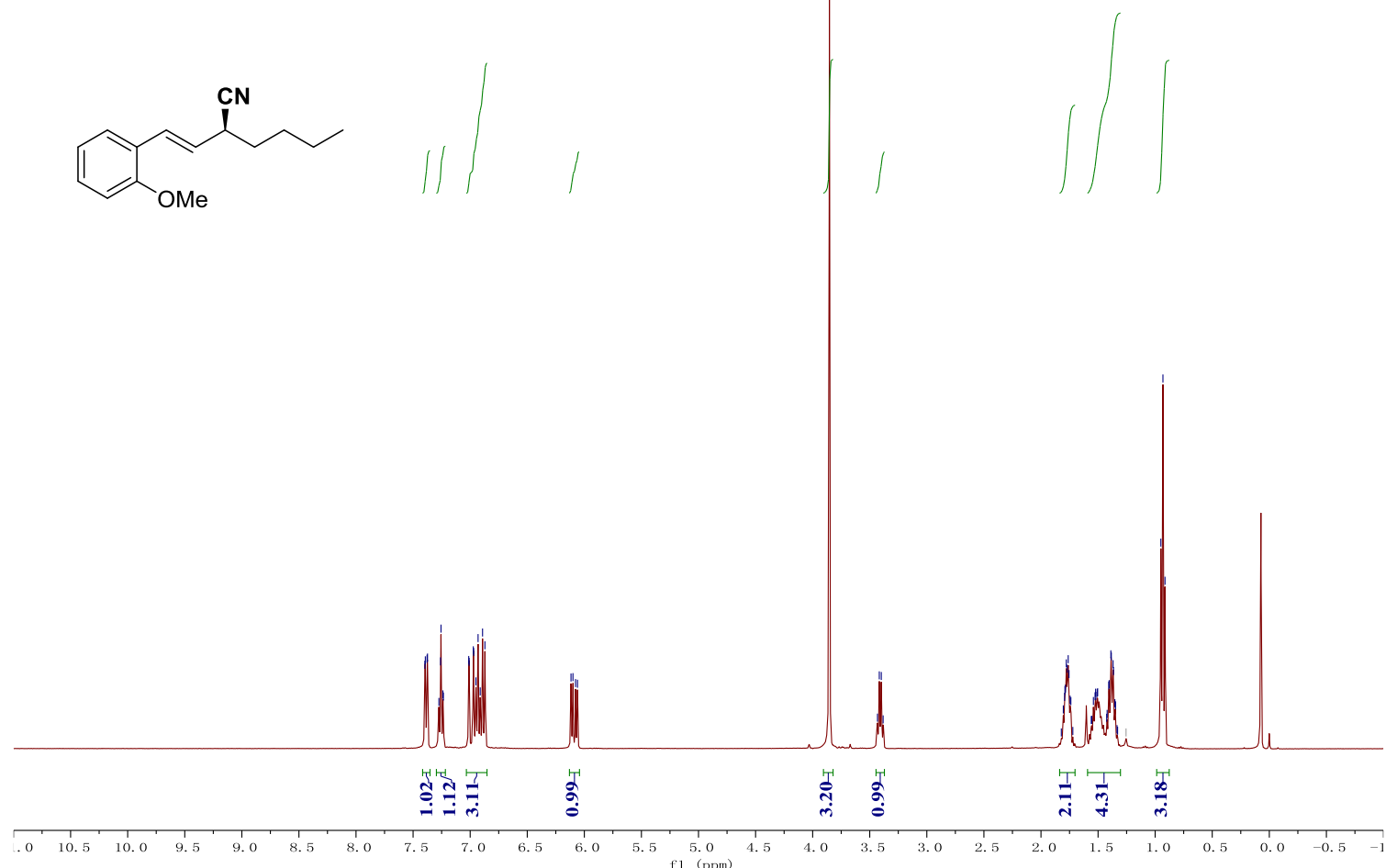

${ }^{13} \mathrm{C}$ NMR spectrum of compound $4 \mathrm{fd}\left(100 \mathrm{MHz} \mathrm{CDCl}_{3}\right)$

$1 \mathrm{fd} 788 \mathrm{c} / 8$

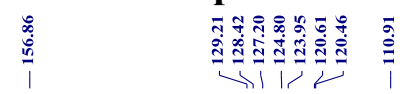

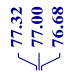

站

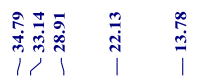<smiles>CCCC[C@H](C#N)/C=C/c1ccccc1OC</smiles>

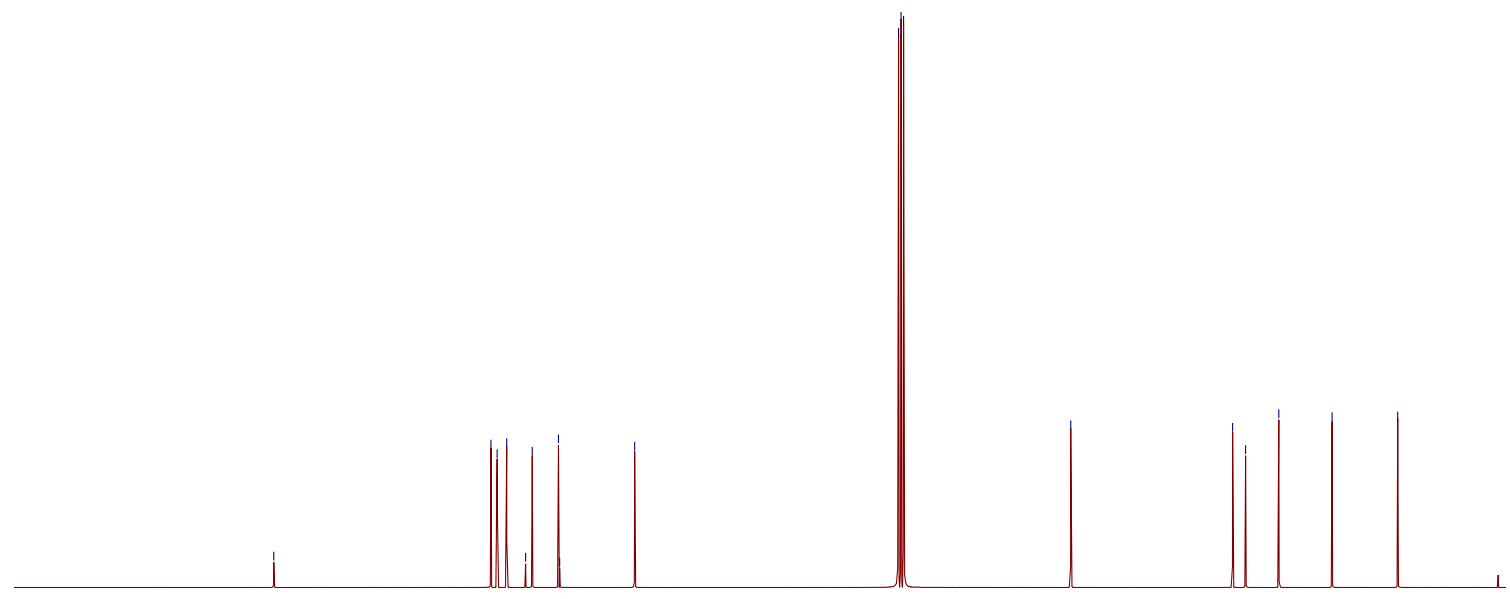

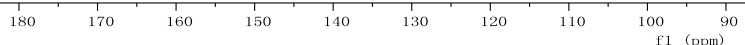


${ }^{1} \mathrm{H}$ NMR spectrum of compound $4 \mathrm{gd}\left(400 \mathrm{MHz}, \mathrm{CDCl}_{3}\right)$

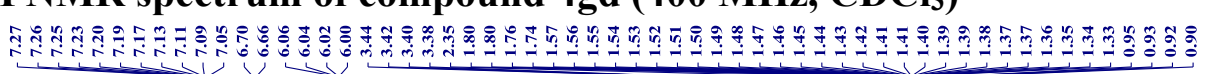
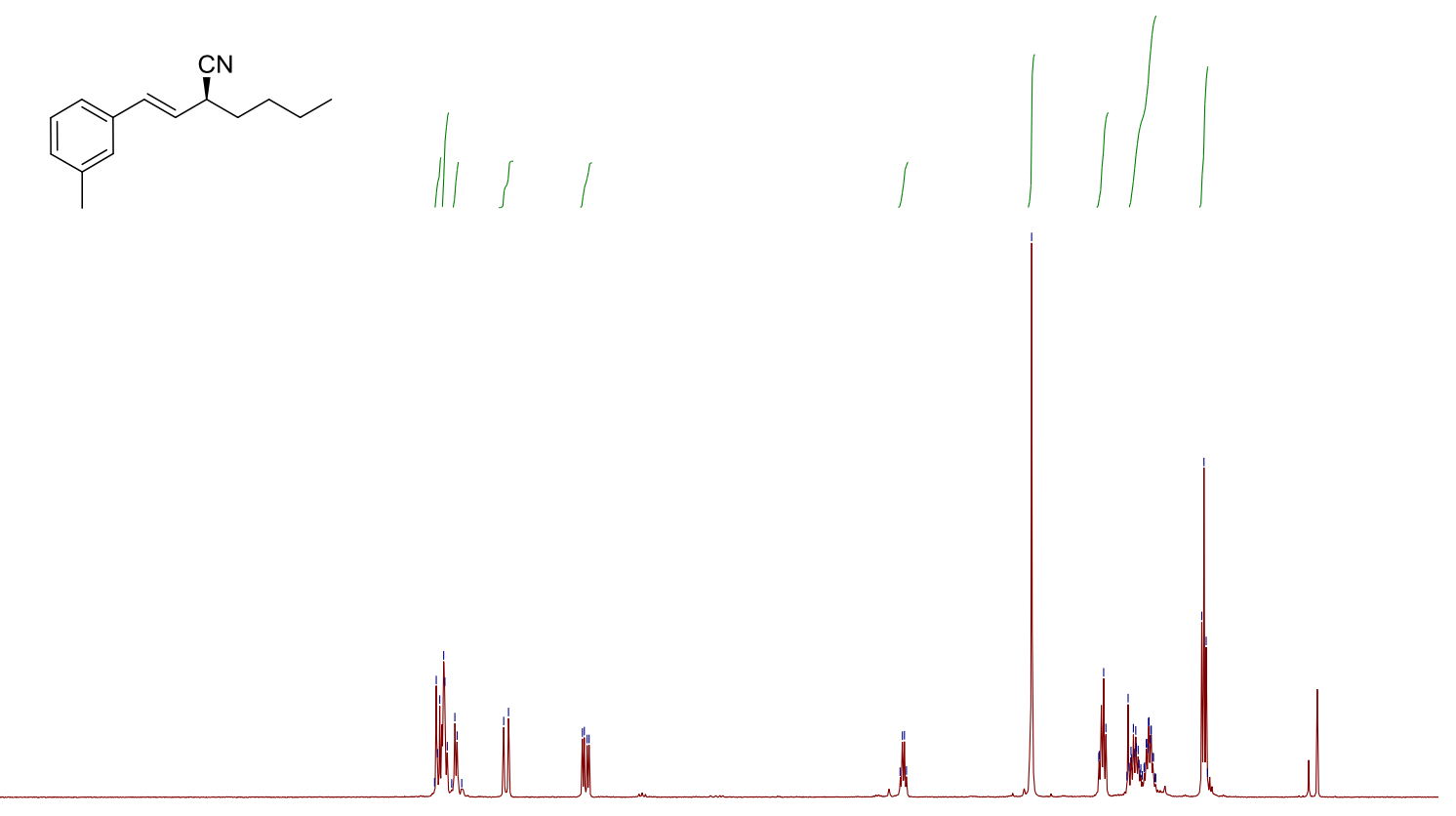

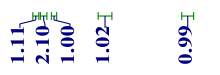

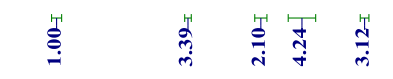

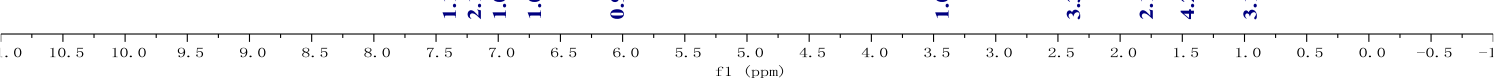

${ }^{13} \mathrm{C}$ NMR spectrum of compound $4 \mathrm{gd}\left(100 \mathrm{MHz}, \mathrm{CDCl}_{3}\right)$

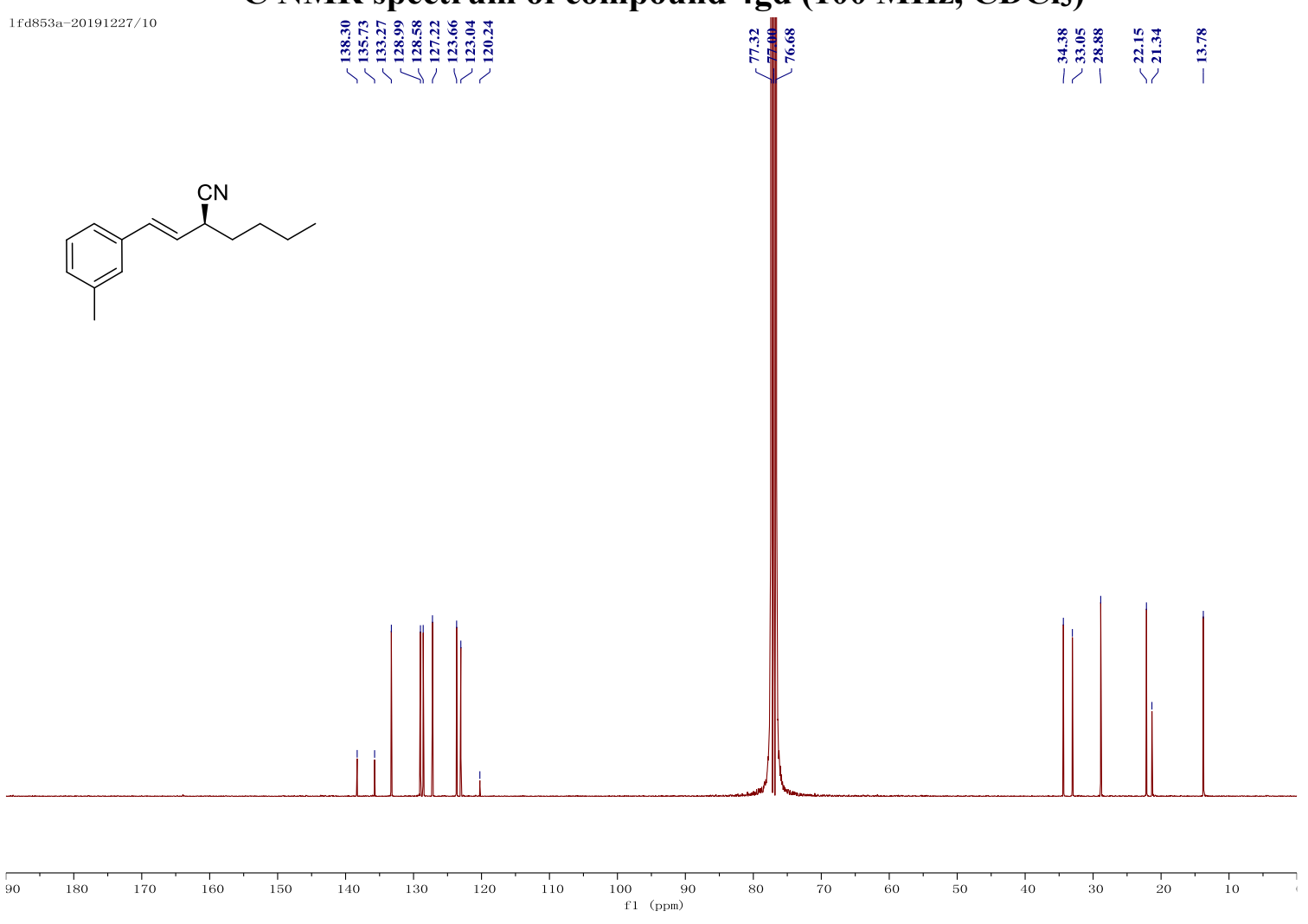

S48 
${ }^{1} \mathrm{H}$ NMR spectrum of compound $4 \mathrm{hd}\left(400 \mathrm{MHz} \mathrm{CDCl}_{3}\right)$

1fd853b-20191227/1<smiles>CCCCC(C#N)C=Cc1cccc(F)c1</smiles>
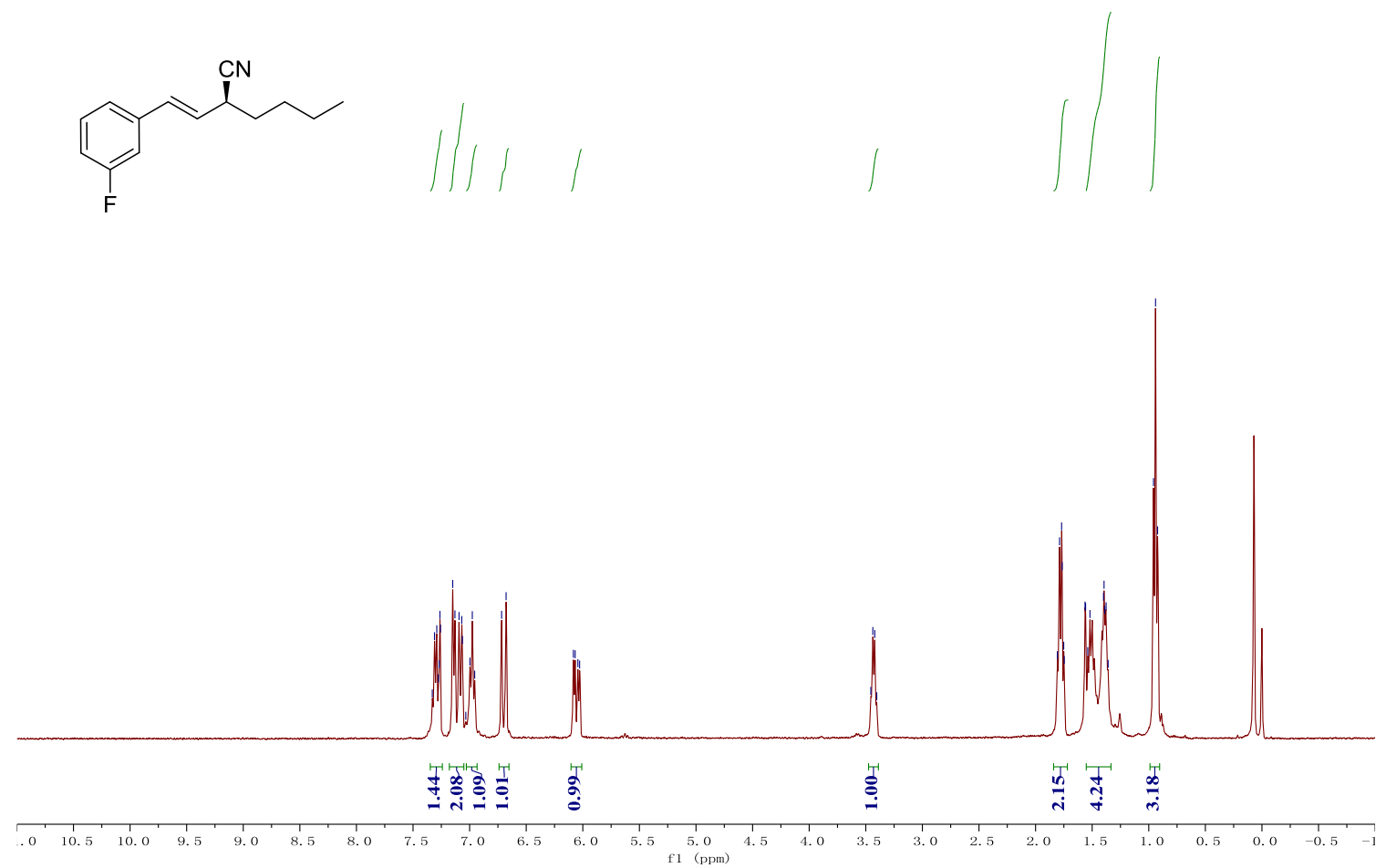

${ }^{13} \mathrm{C}$ NMR spectrum of compound $4 \mathrm{hd}\left(100 \mathrm{MHz}, \mathrm{CDCl}_{3}\right)$

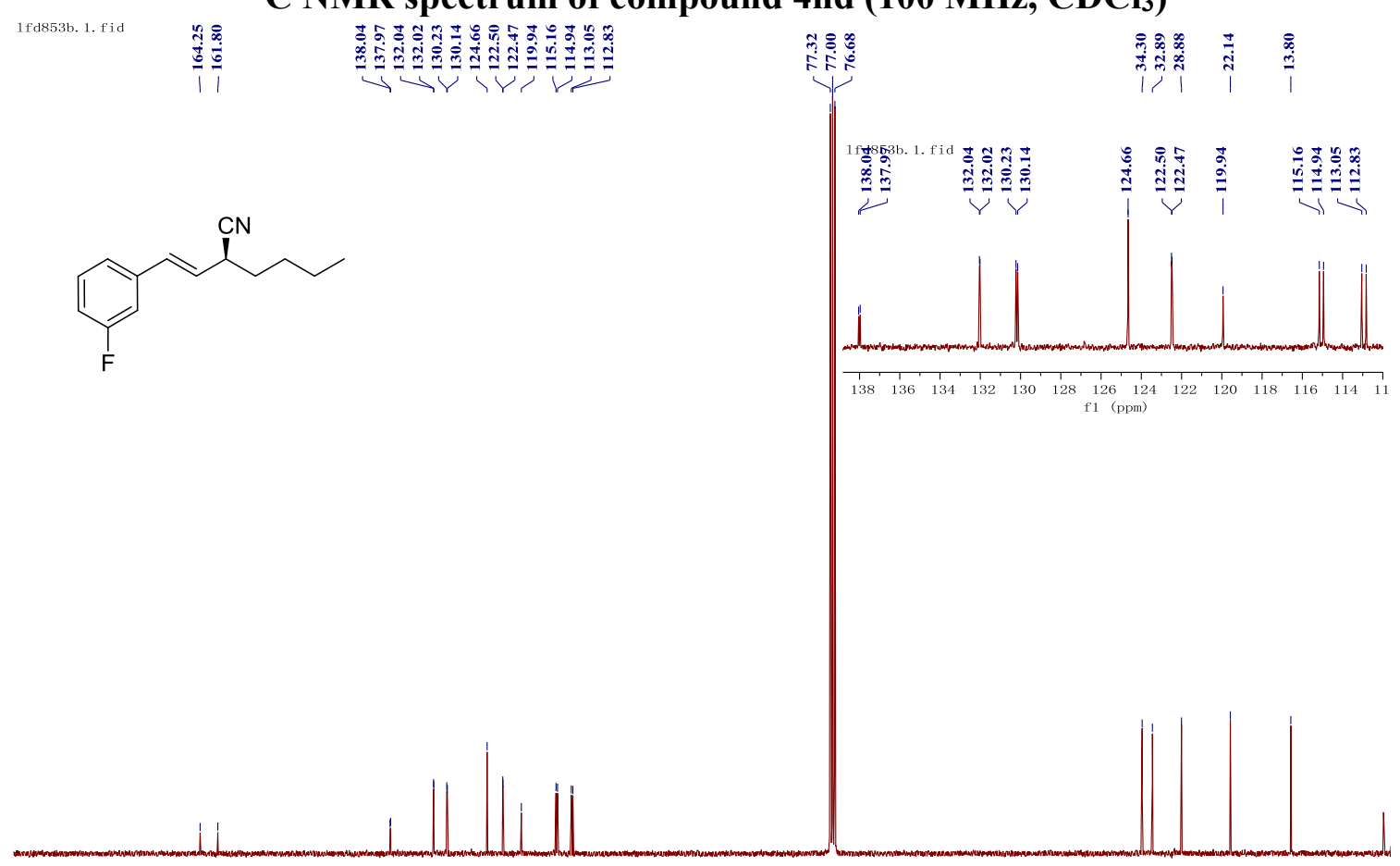

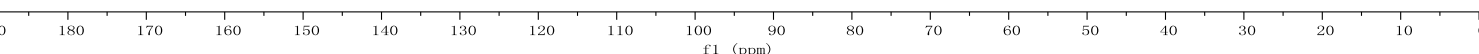


${ }^{19}$ F NMR spectrum of compound 4 hd (376 $\left.\mathrm{MHz}, \mathrm{CDCl}_{3}\right)$

1fd853b. 2. fid

$\stackrel{\stackrel{9}{i}}{i}$
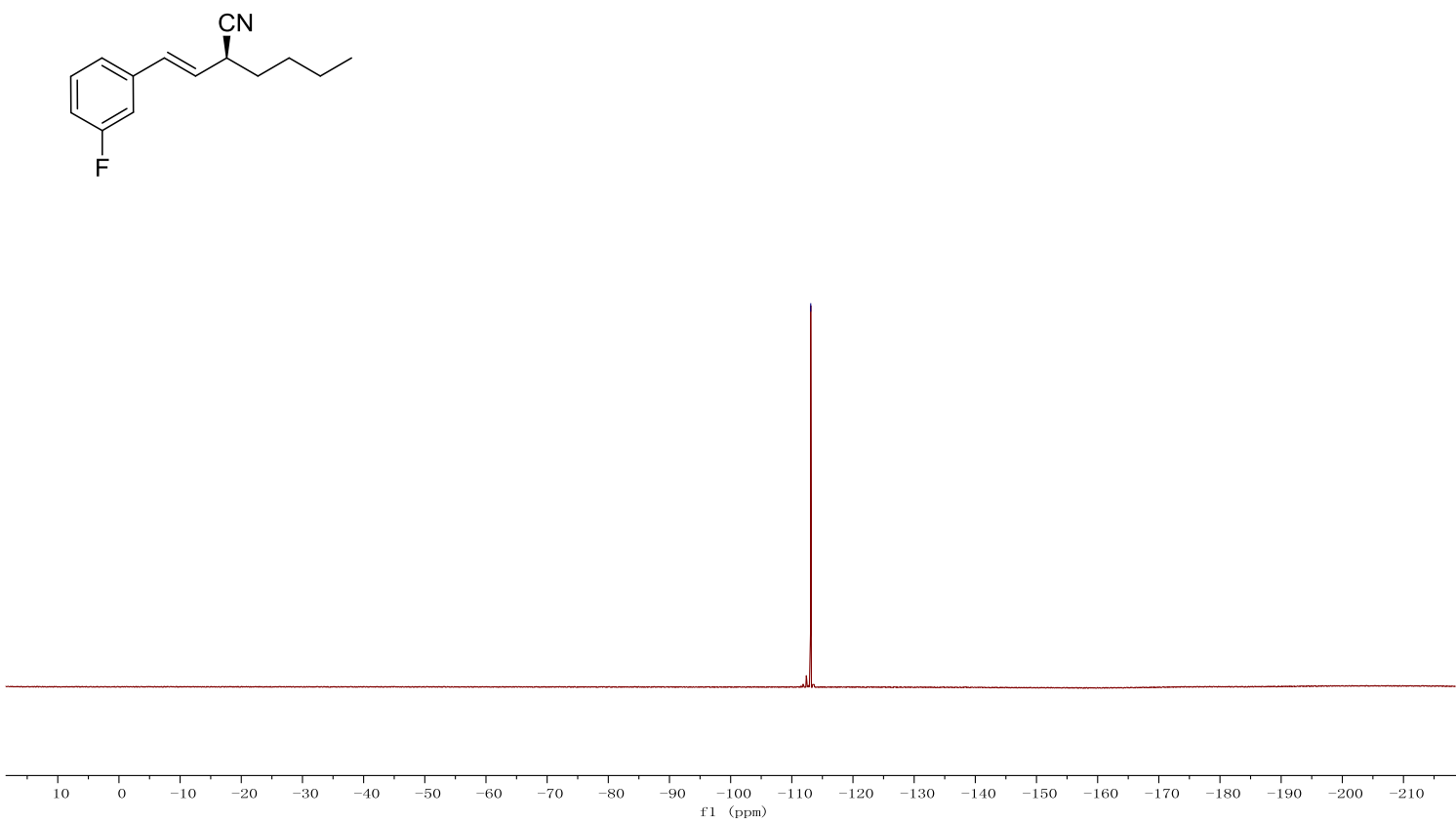

${ }^{1} \mathrm{H}$ NMR spectrum of compound $4 \mathrm{id}\left(400 \mathrm{MHz}, \mathrm{CDCl}_{3}\right)$

If $840 \mathrm{c}-20191220 / 1$

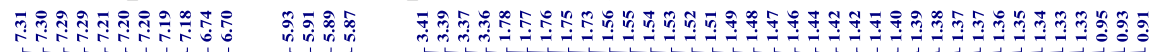
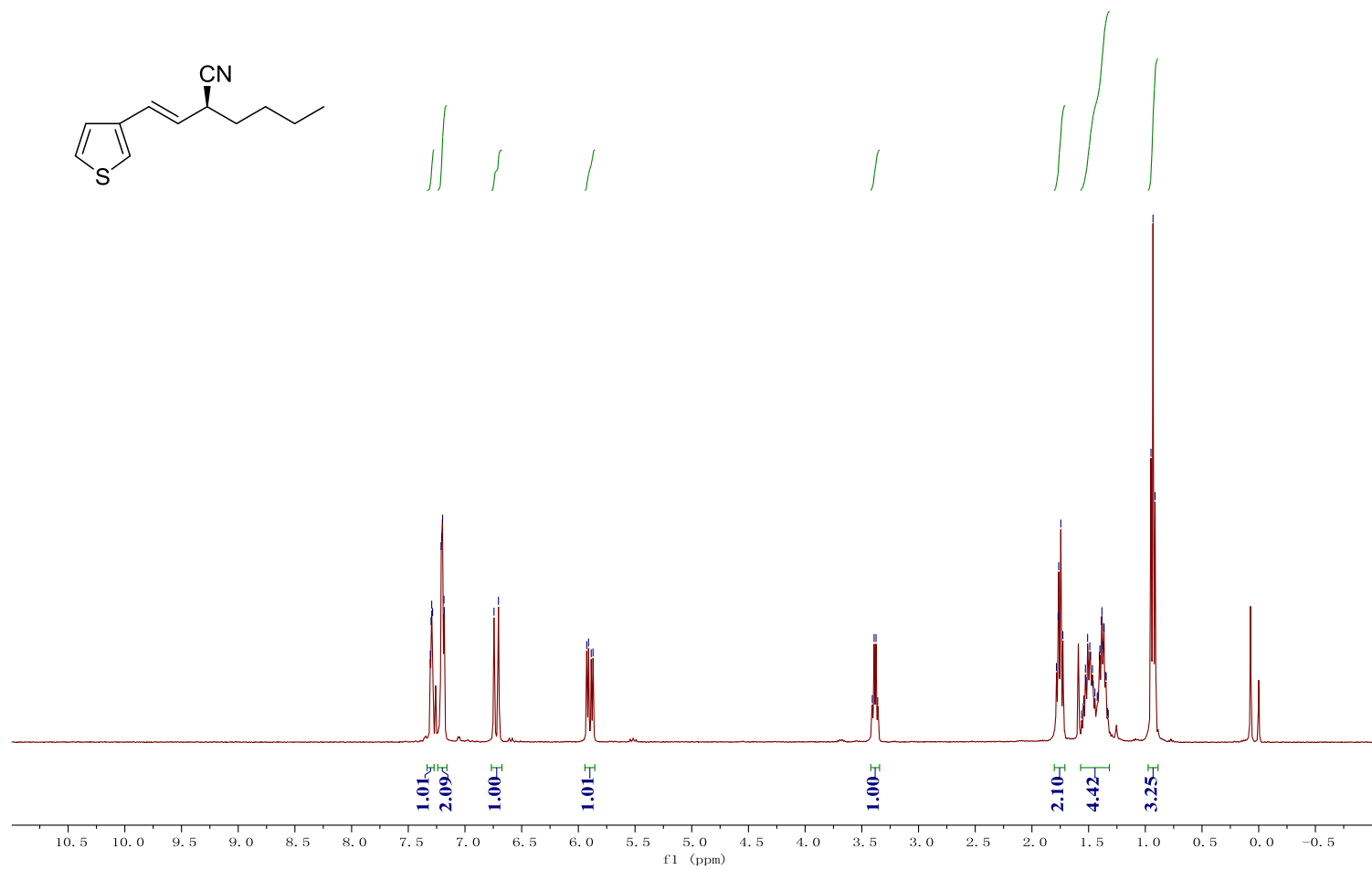

$\mathrm{S} 50$ 
${ }^{13} \mathrm{C}$ NMR spectrum of compound $4 \mathrm{id}\left(100 \mathrm{MHz}, \mathrm{CDCl}_{3}\right)$

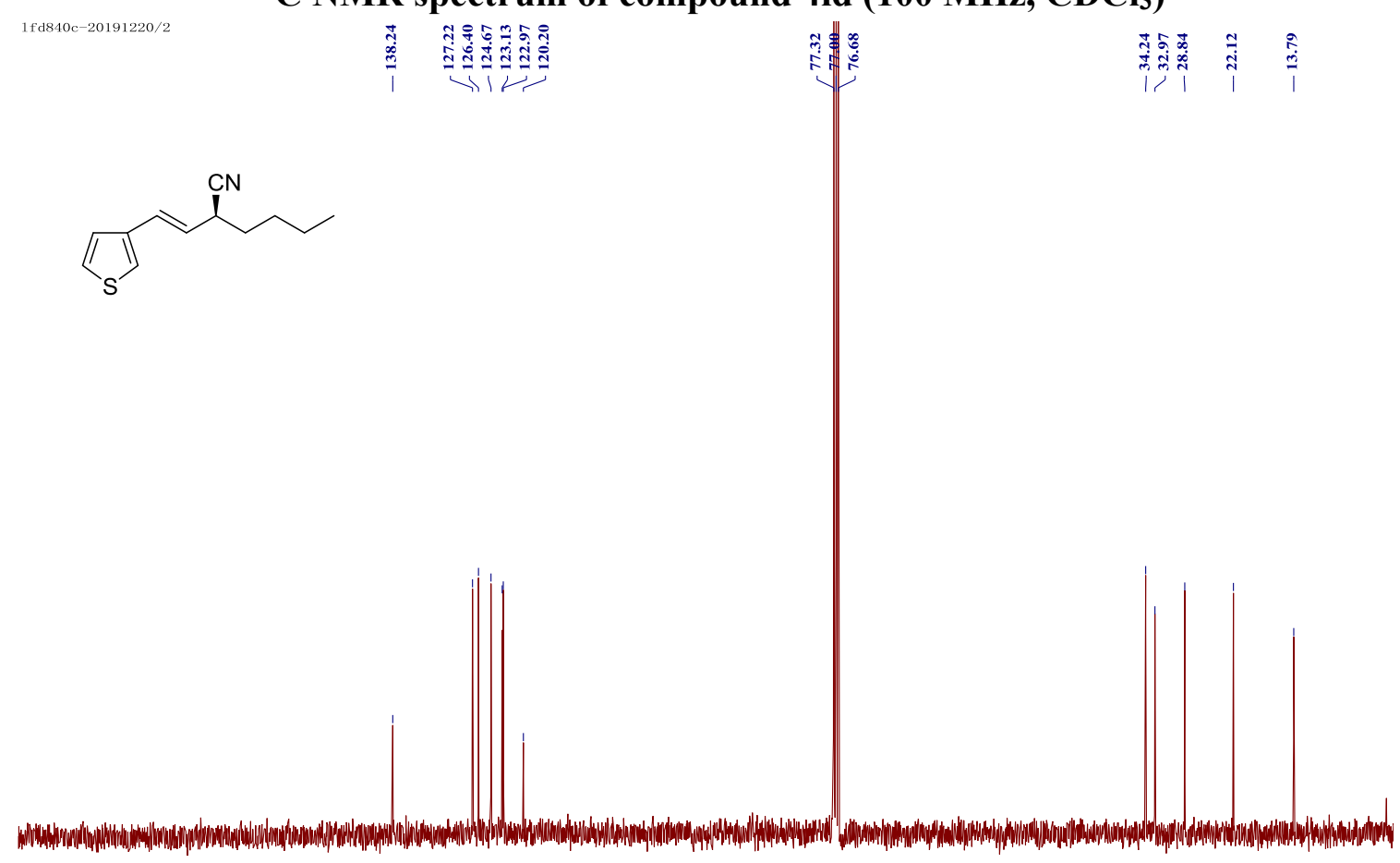

\section{${ }^{1} \mathrm{H}$ NMR spectrum of compound $4 \mathrm{jd}\left(400 \mathrm{MHz}, \mathrm{CDCl}_{3}\right)$}

1fa1199b-2021028/1<smiles>CCCCC(C#N)/C=C/c1ccoc1</smiles><smiles>C1=CC2CCCCC2C1</smiles>

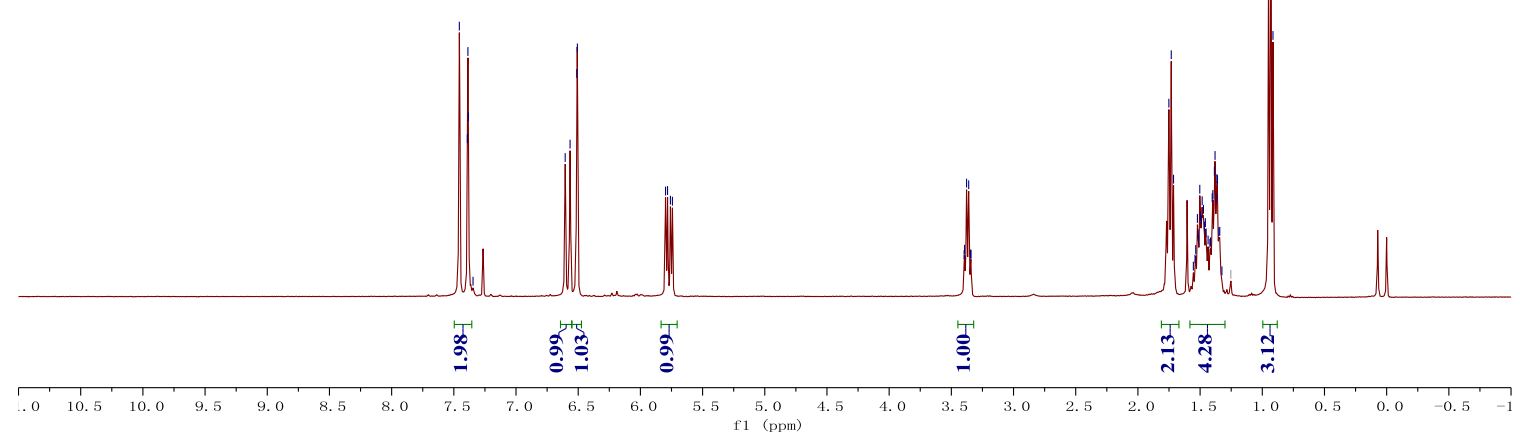


${ }^{13} \mathrm{C}$ NMR spectrum of compound 4jd (100 $\left.\mathrm{MHz}, \mathrm{CDCl}_{3}\right)$
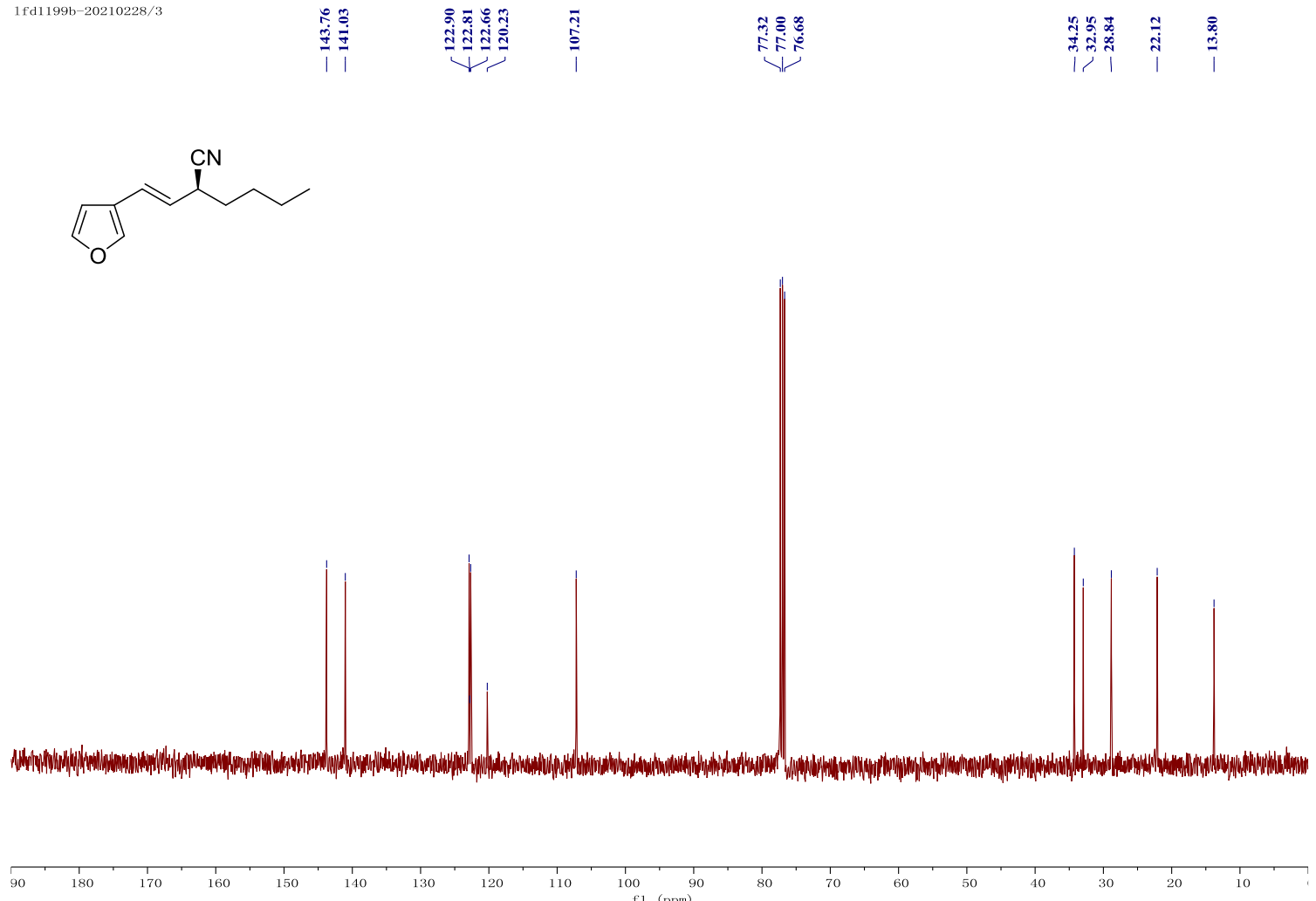

${ }^{1} \mathrm{H}$ NMR spectrum of compound $4 \mathrm{kd}\left(400 \mathrm{MHz} \mathrm{CDCl}_{3}\right)$
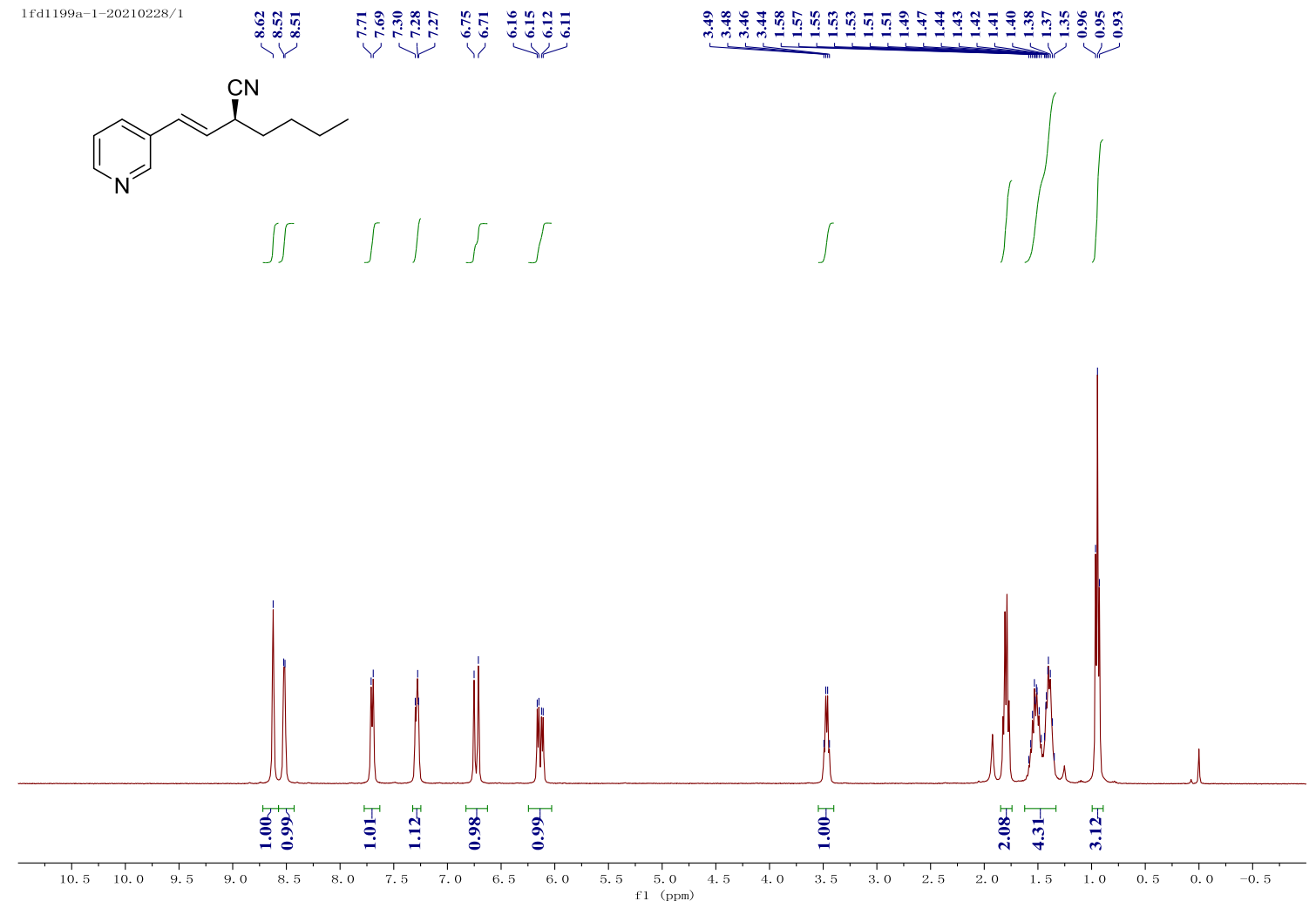
${ }^{13} \mathrm{C}$ NMR spectrum of compound $4 \mathrm{kd}\left(100 \mathrm{MHz}, \mathrm{CDCl}_{3}\right)$

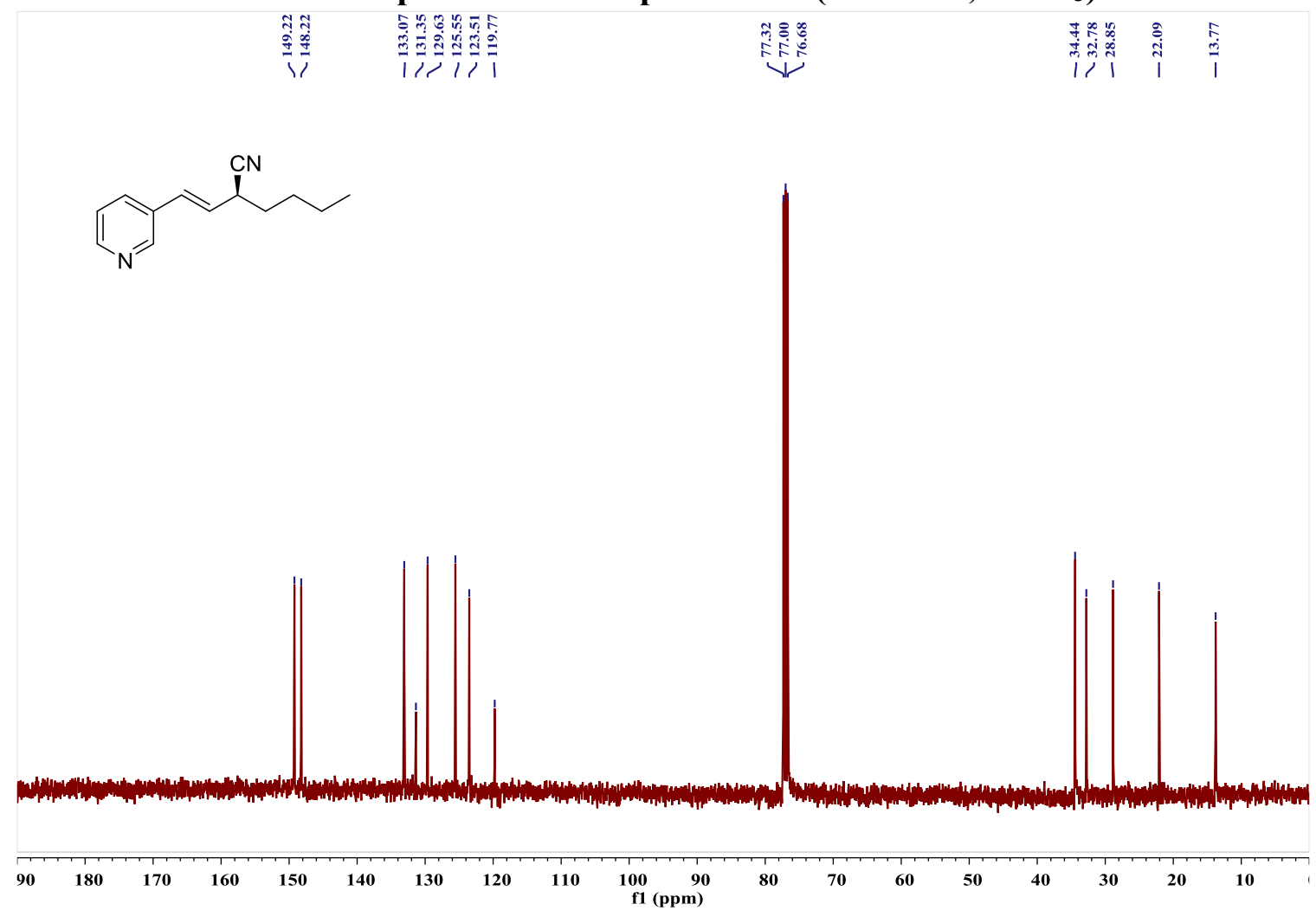

${ }^{1} \mathrm{H}$ NMR spectrum of compound $4 \mathrm{ld}\left(400 \mathrm{MHz}, \mathrm{CDCl}_{3}\right)$

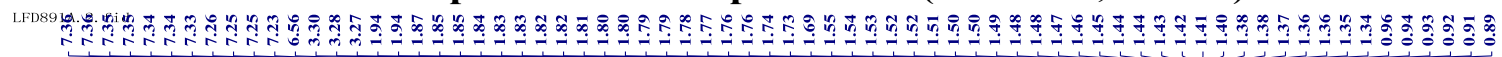<smiles></smiles>
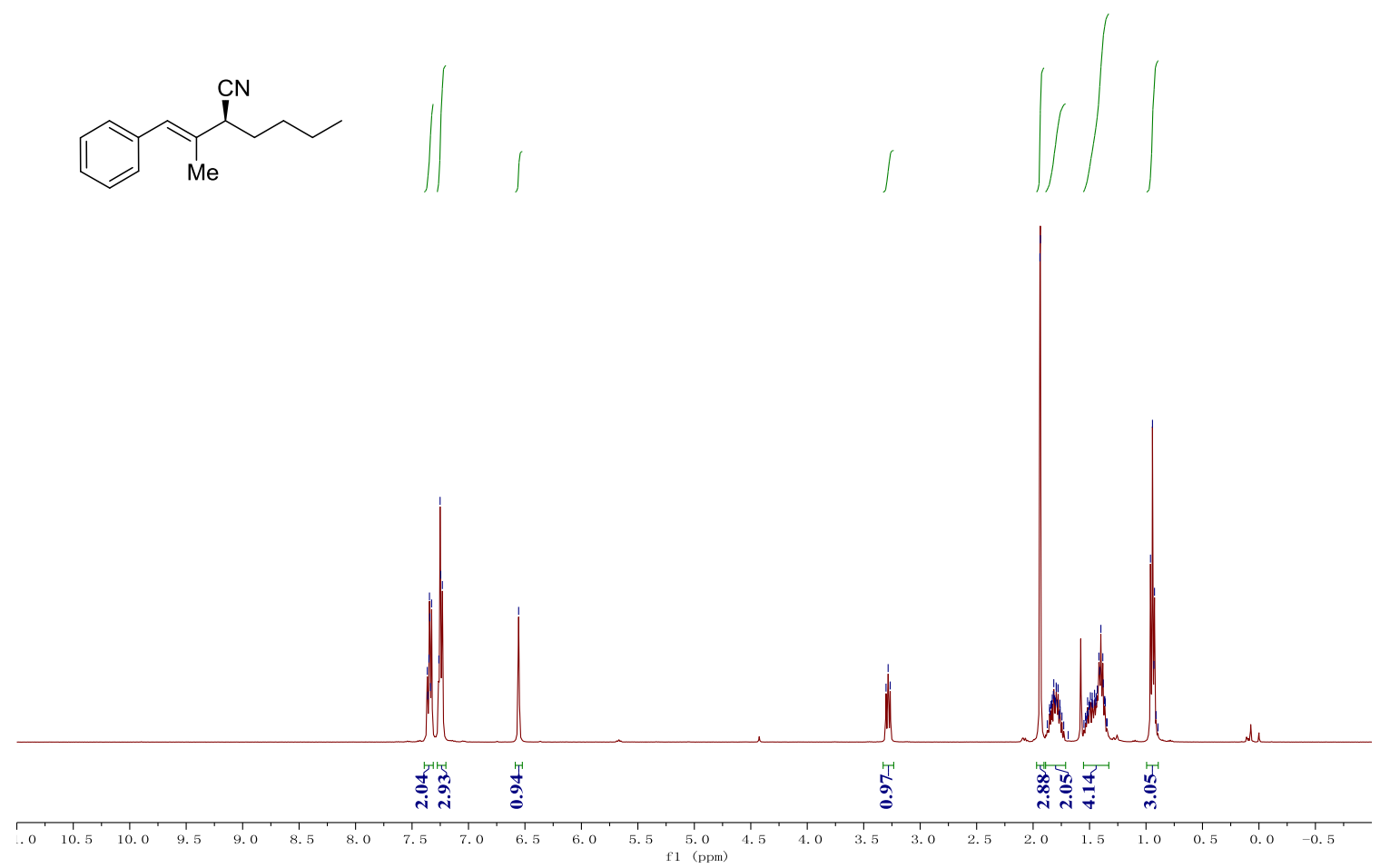
${ }^{13} \mathrm{C}$ NMR spectrum of compound $4 \mathrm{ld}\left(100 \mathrm{MHz}, \mathrm{CDCl}_{3}\right)$
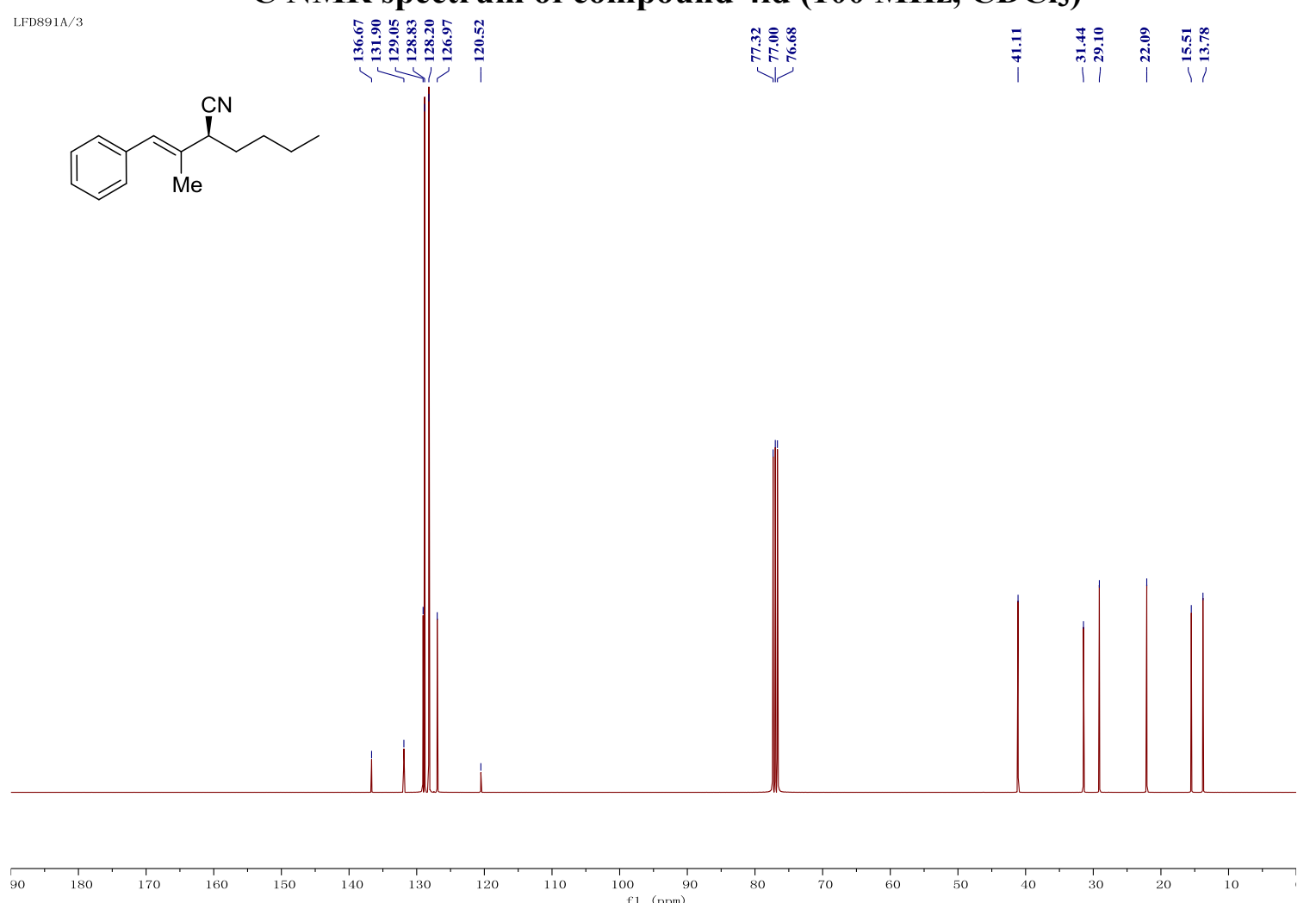

${ }^{1} \mathrm{H}$ NMR spectrum of compound $4 \mathrm{md}\left(400 \mathrm{MHz} \mathrm{CDCl}_{3}\right)$<smiles>CCCC[C@H](C#N)/C(Br)=C/c1ccccc1</smiles><smiles>CC1C=CCCC1</smiles>

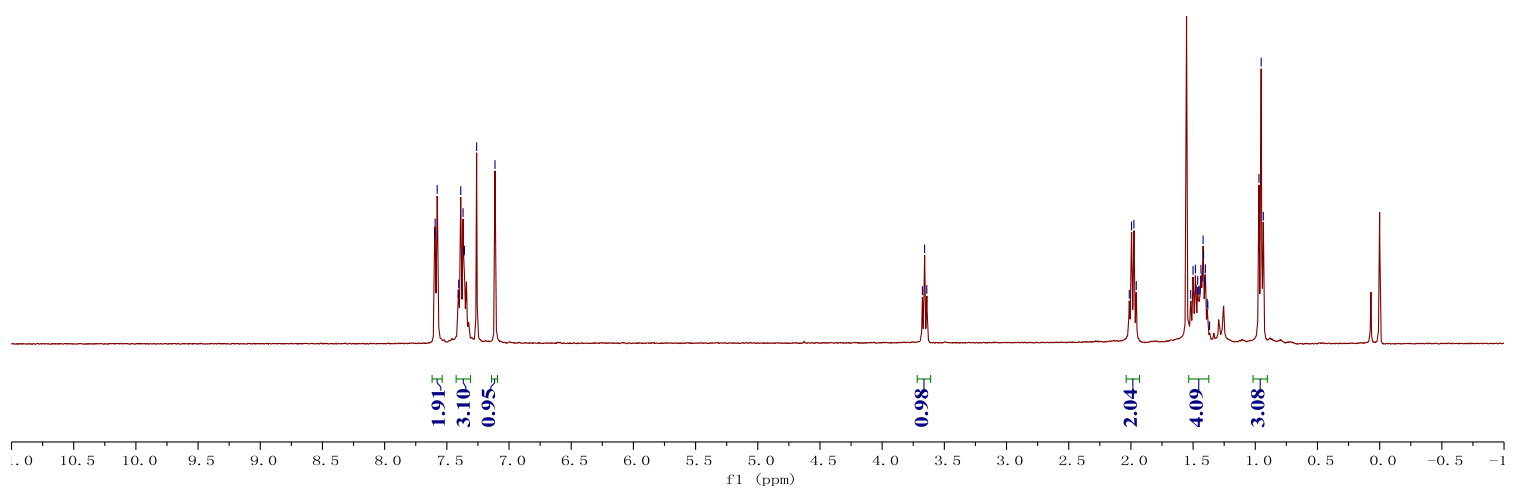


${ }^{13} \mathrm{C}$ NMR spectrum of compound $4 \mathrm{md}\left(100 \mathrm{MHz}, \mathrm{CDCl}_{3}\right)$
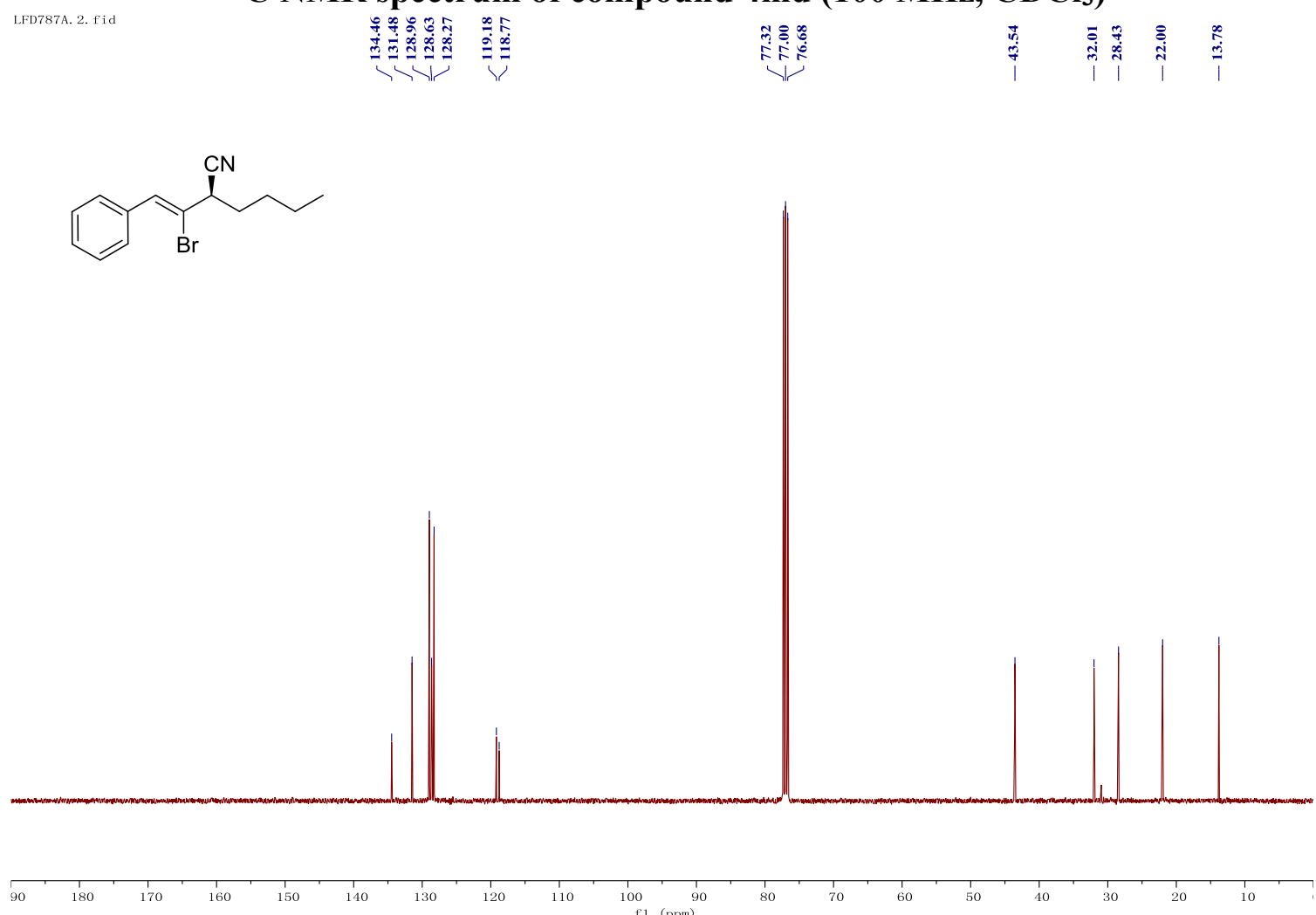

${ }^{1} \mathrm{H}$ NMR spectrum of compound $4 \mathrm{nd}\left(400 \mathrm{MHz} \mathrm{CDCl}_{3}\right)$

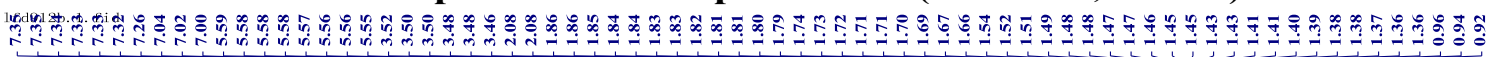

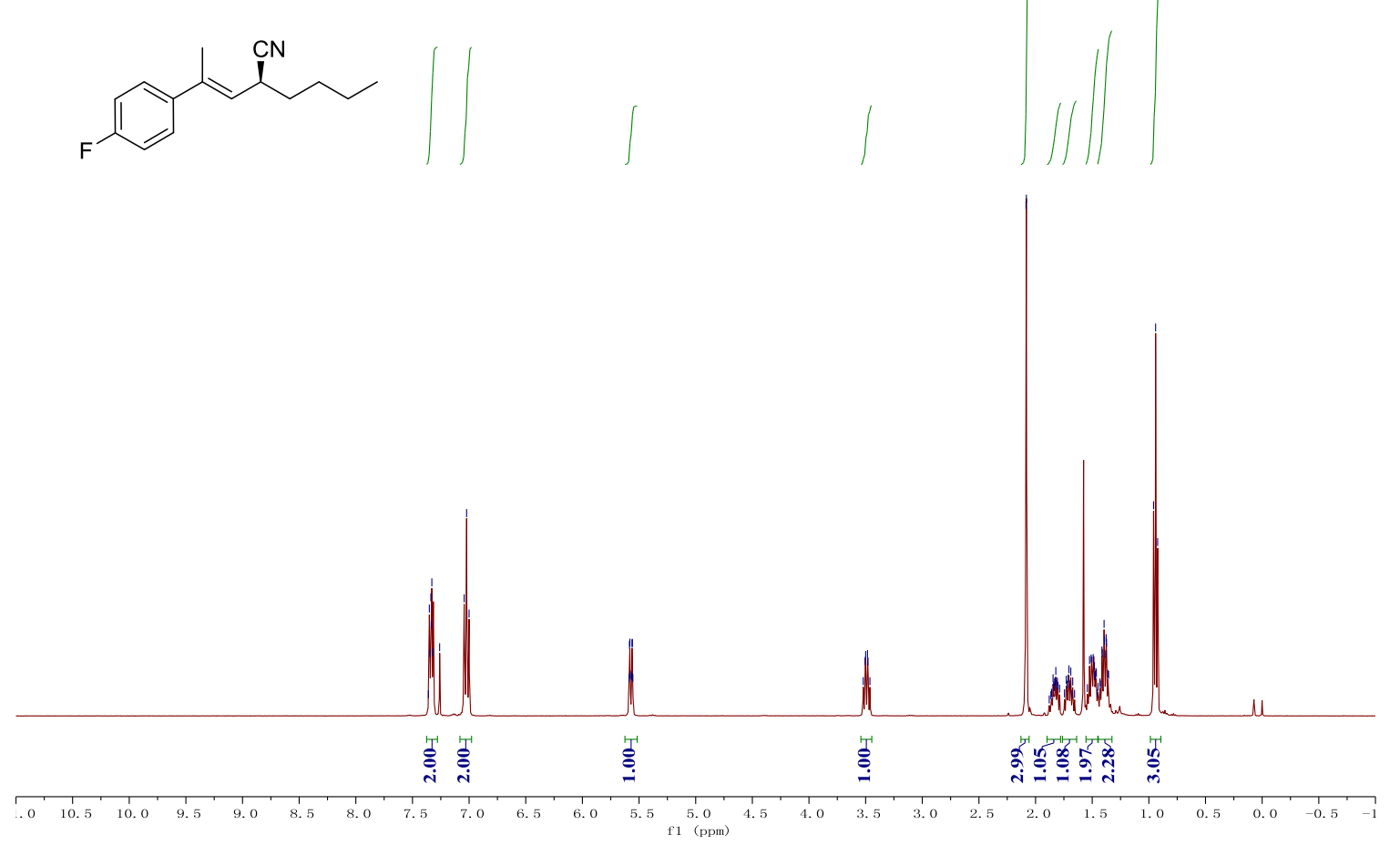


${ }^{13} \mathrm{C}$ NMR spectrum of compound 4nd (100 $\left.\mathrm{MHz}, \mathrm{CDCl}_{3}\right)$
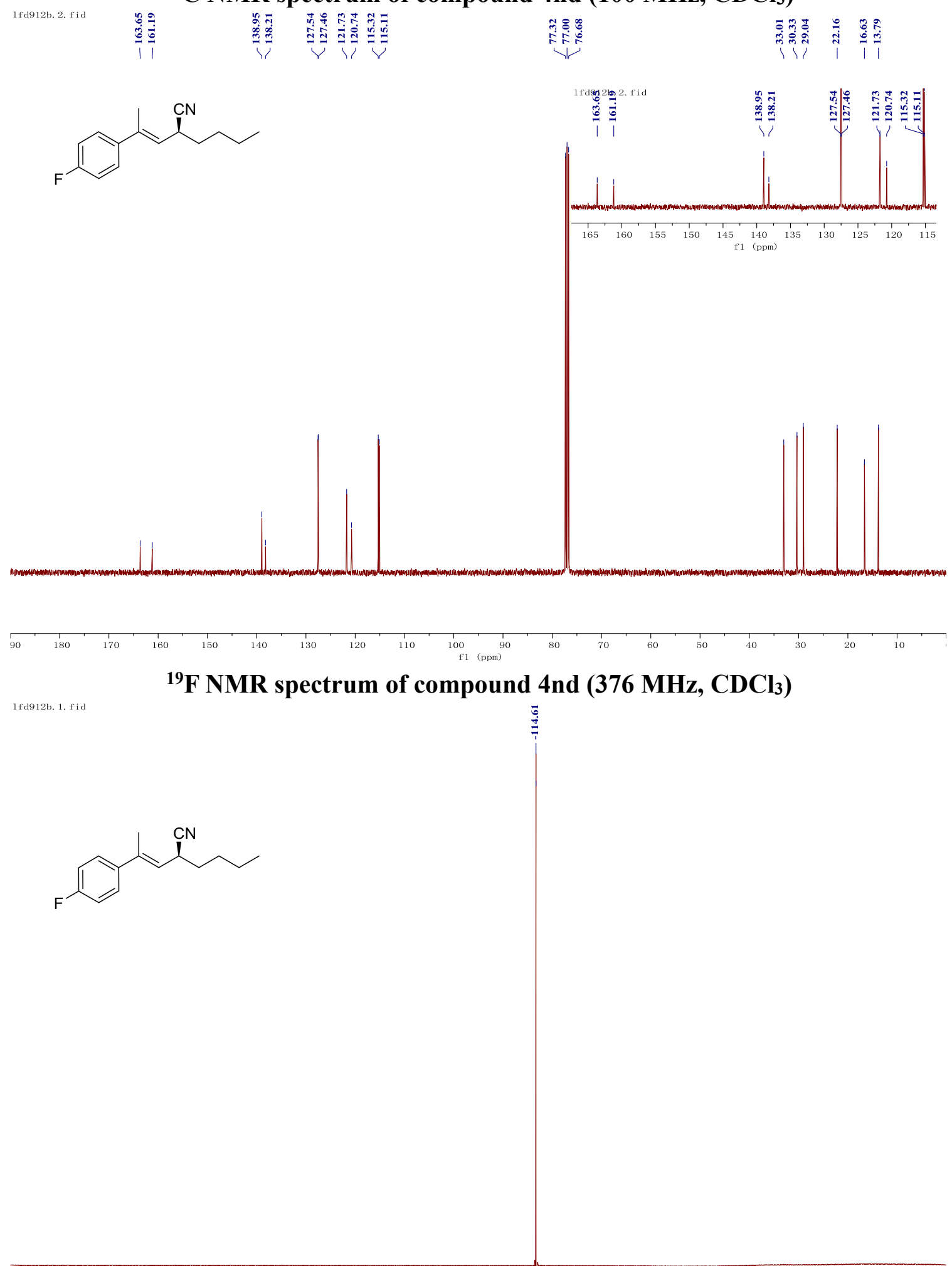

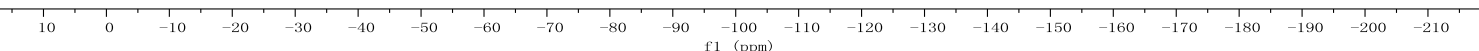


${ }^{1} \mathrm{H}$ NMR spectrum of compound $40 d\left(400 \mathrm{MHz}, \mathrm{CD}_{3} \mathrm{OD}\right)$

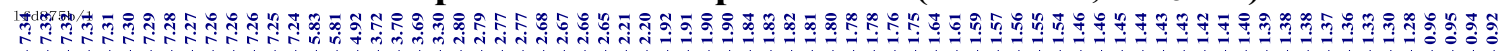<smiles>N#CC1=CC(c2ccccc2)CCC1C(=O)Nc1ccccc1</smiles>
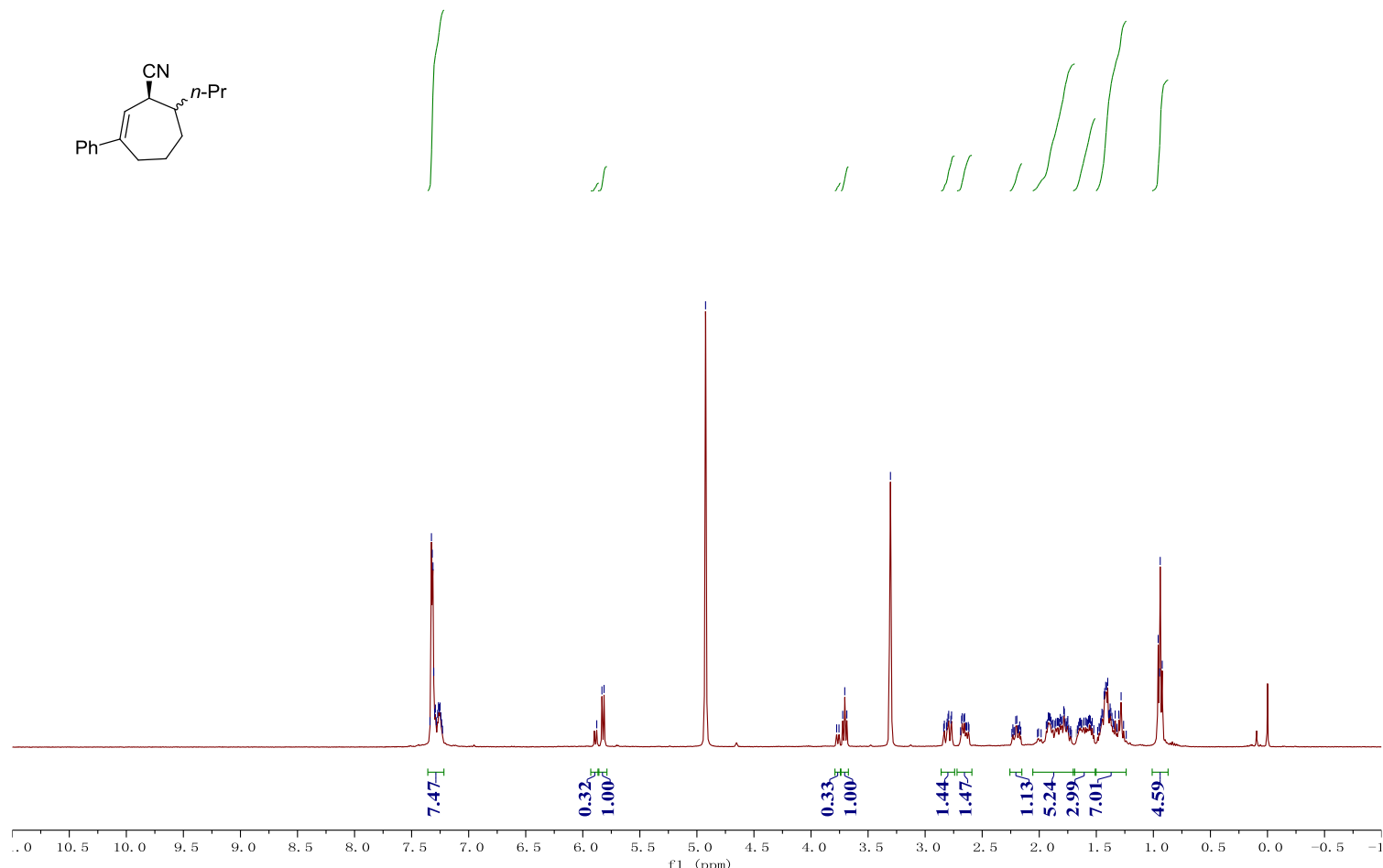

${ }^{13} \mathrm{C}$ NMR spectrum of compound $40 d\left(150 \mathrm{MHz} \mathrm{CDCl}_{3}\right)$

20200721-1fd891b-2-C13

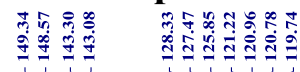

\%

管:

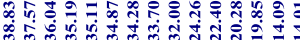

$\overbrace{}^{n-P r}$

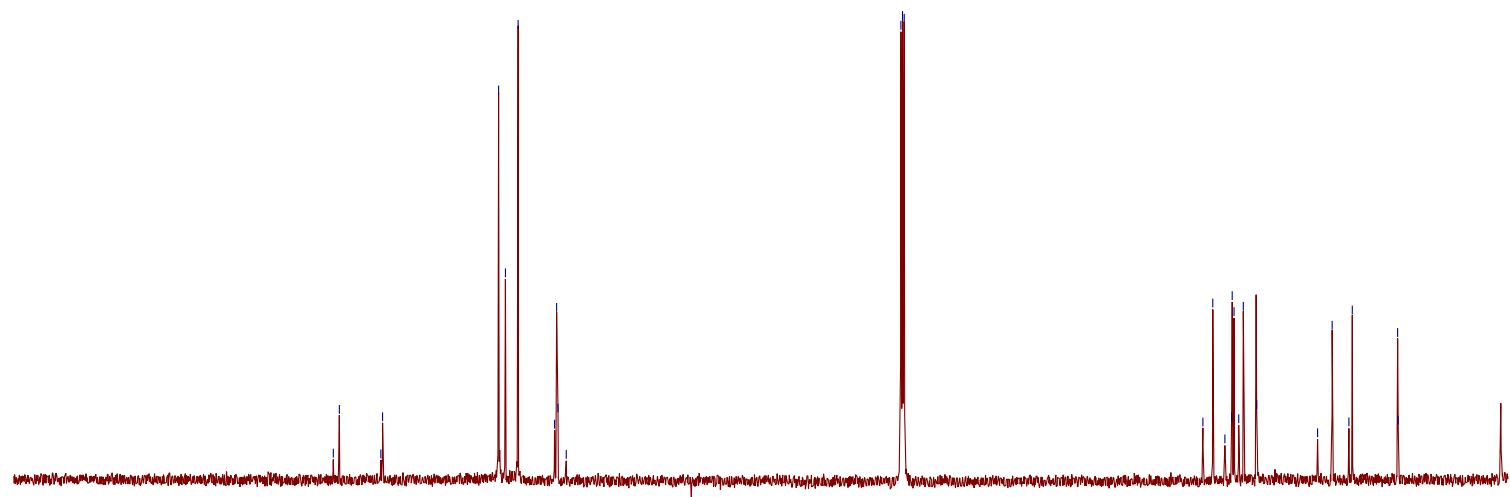


${ }^{1} \mathrm{H}$ NMR spectrum of compound $4 \mathrm{qd}\left(400 \mathrm{MHz}, \mathrm{CDCl}_{3}\right)$

1 fd1193-3-20210226/1

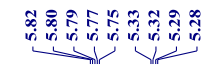

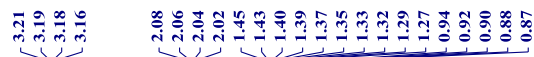
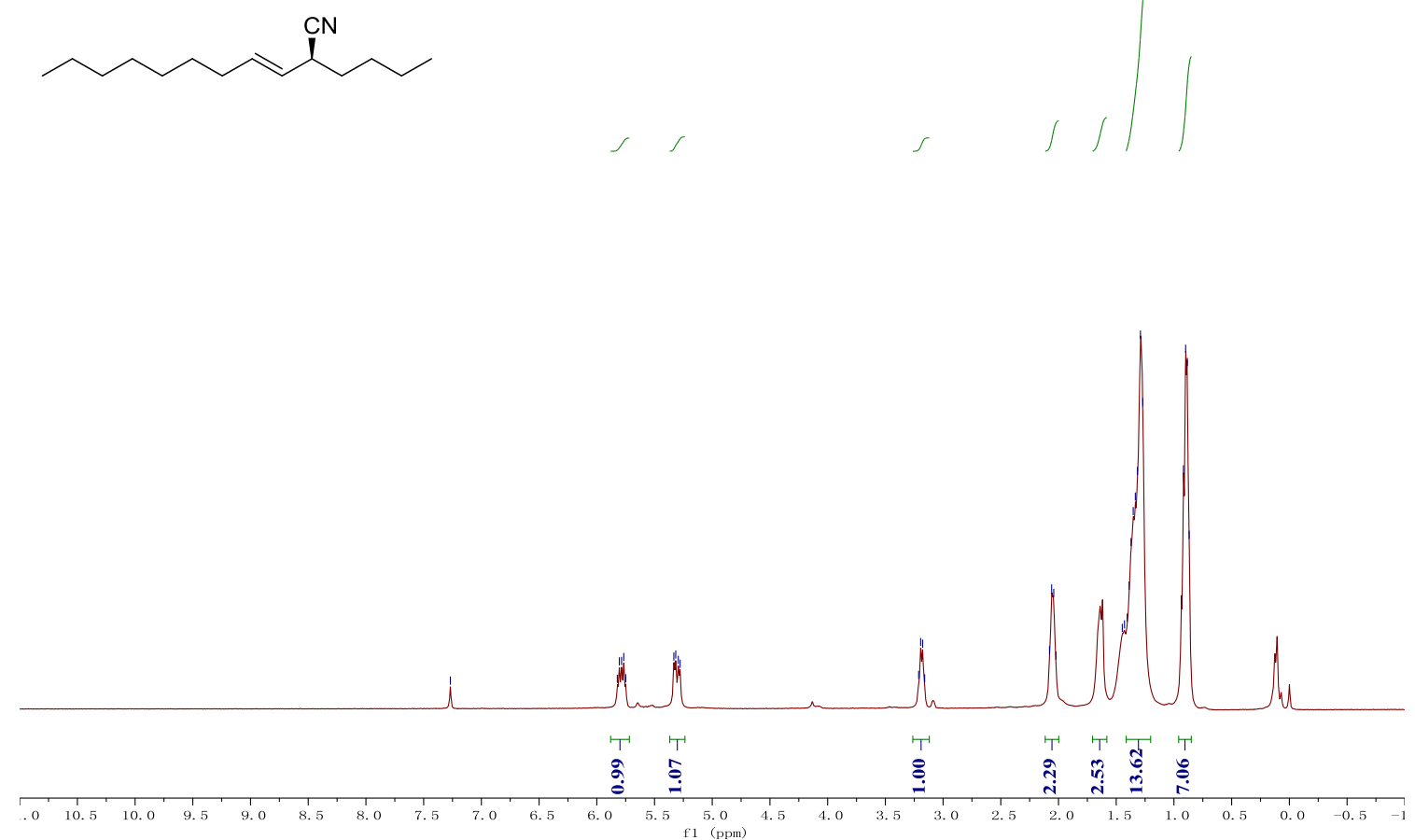

${ }^{13} \mathrm{C}$ NMR spectrum of compound $4 \mathrm{qd}\left(100 \mathrm{MHz}, \mathrm{CDCl}_{3}\right)$

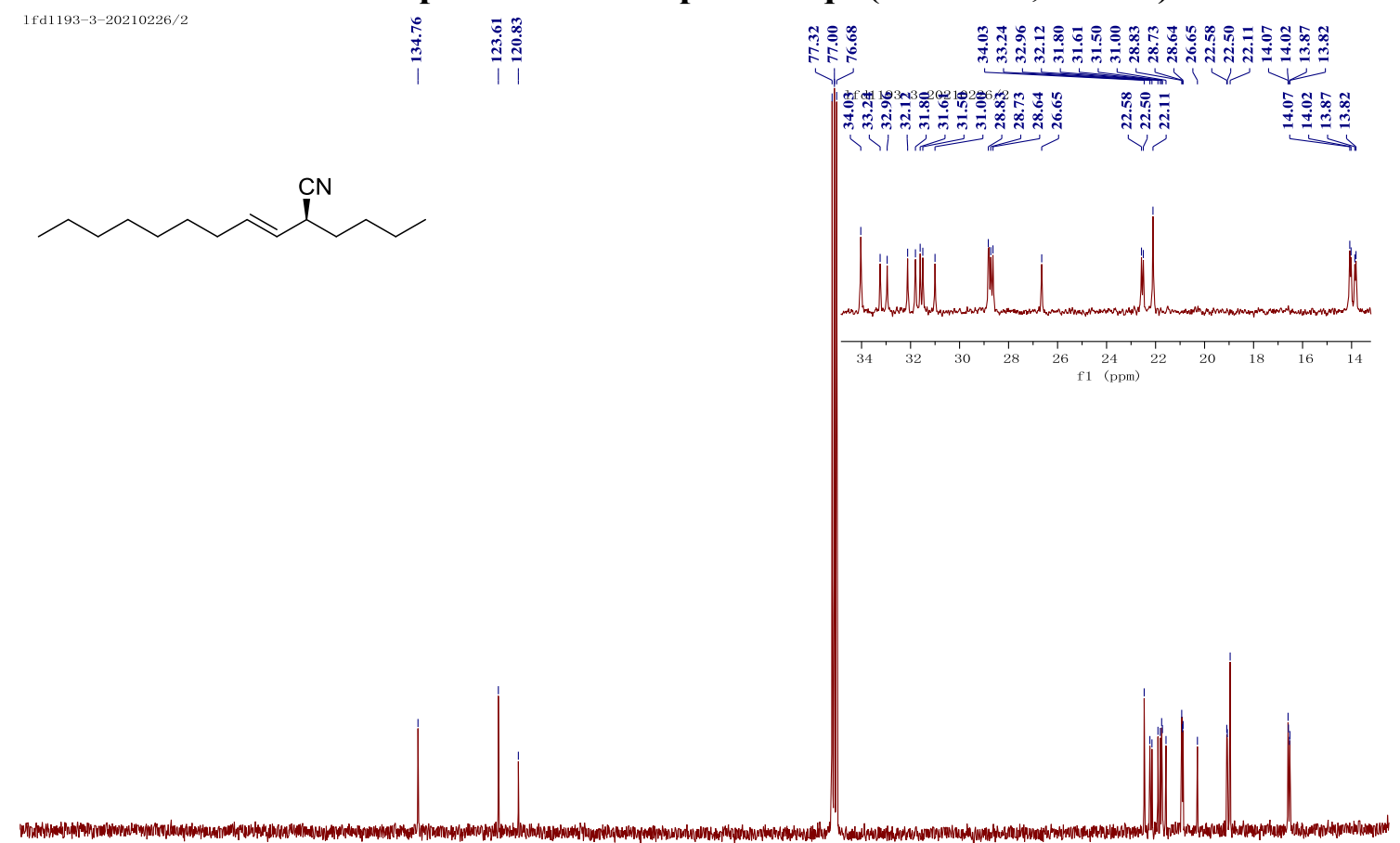


${ }^{1} \mathrm{H}$ NMR spectrum of compound 4aj (600 $\left.\mathrm{MHz}, \mathrm{CDCl}_{3}\right)$

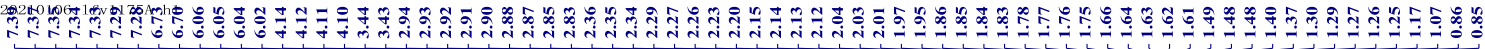

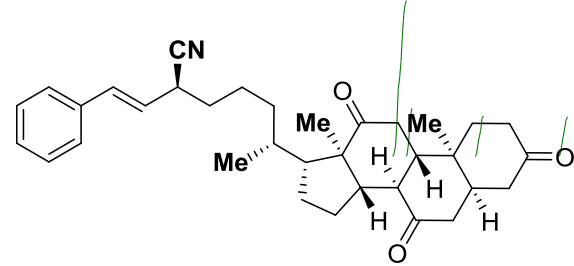
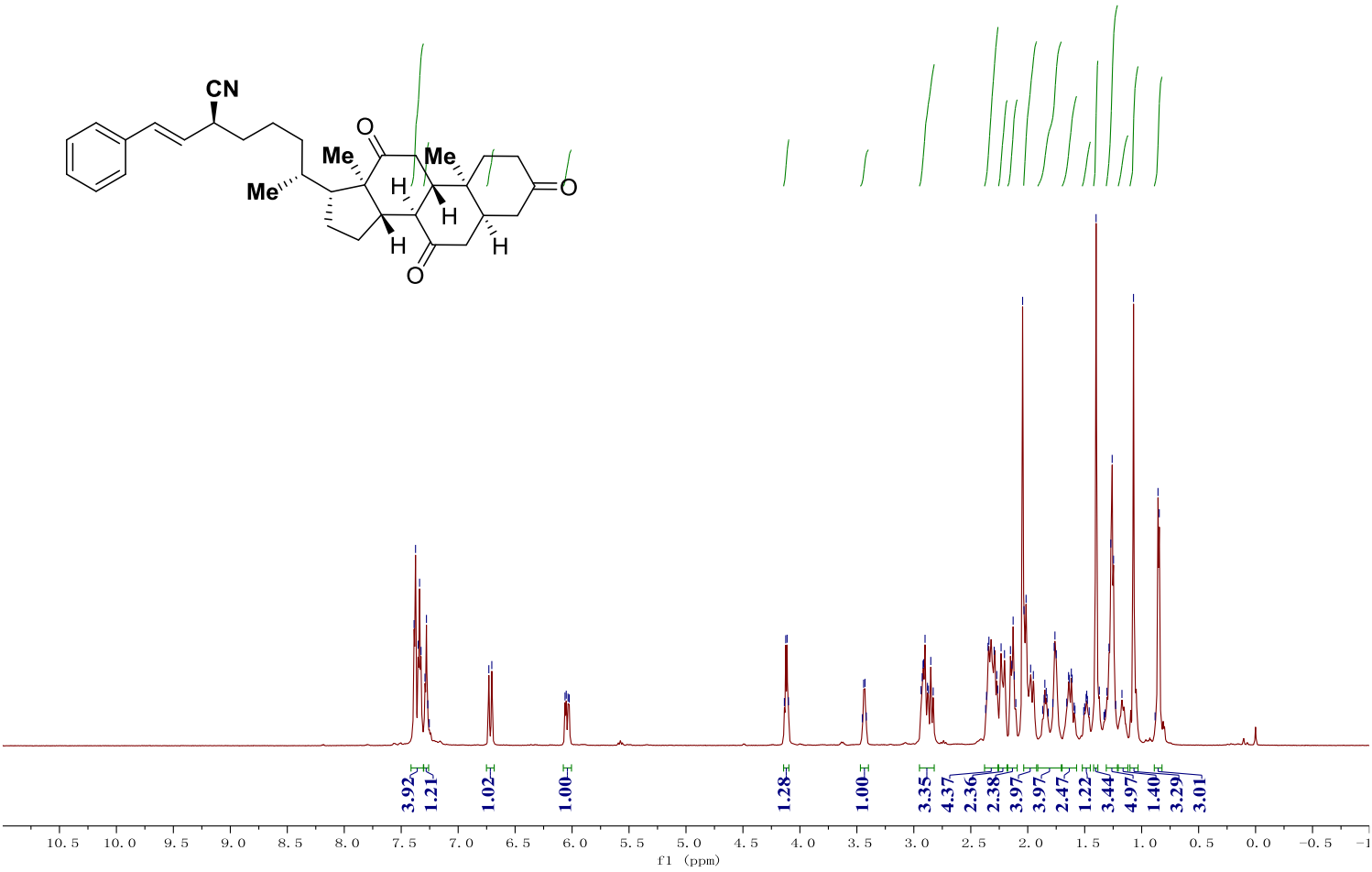

${ }^{13} \mathrm{C}$ NMR spectrum of compound 4aj (100 $\left.\mathrm{MHz}, \mathrm{CDCl}_{3}\right)$

ปู่

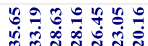

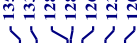

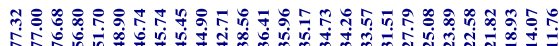

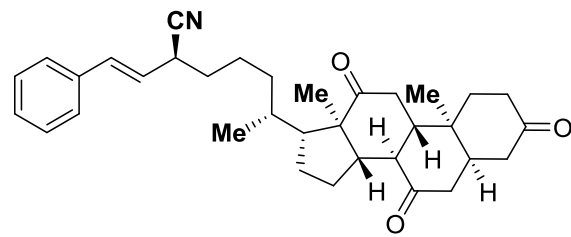

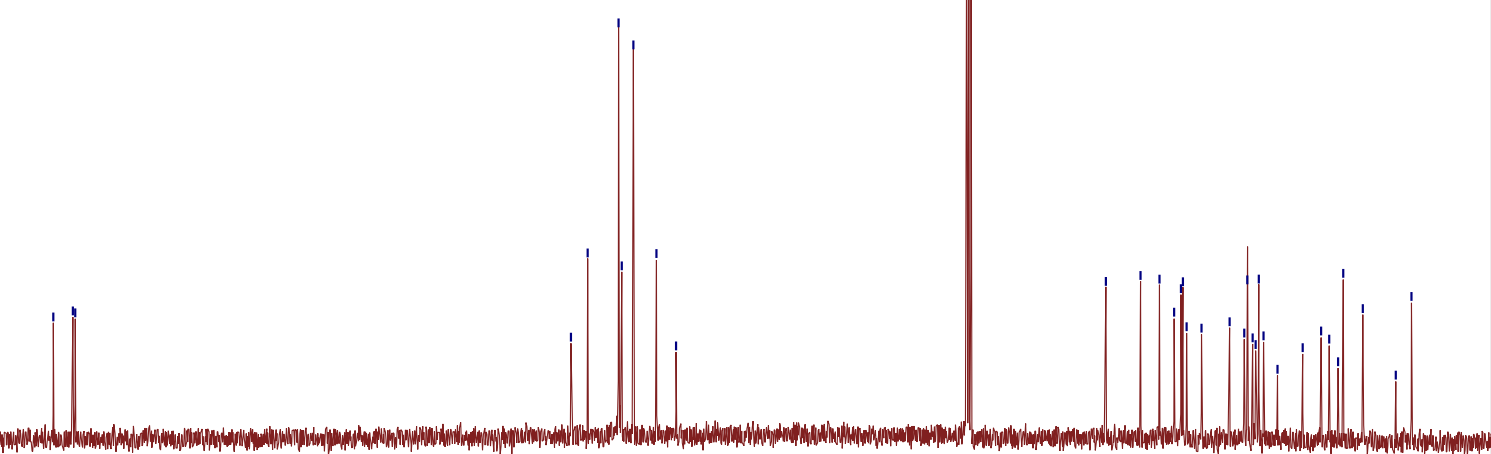

$\begin{array}{llllllllllllllllllllllllllll}20 & 210 & 200 & 190 & 180 & 170 & 160 & 150 & 140 & 130 & 120 & 110 & 100 & 90 & 80 & 70 & 60 & 50 & 40 & 30 & 20 & 10 & \end{array}$ 


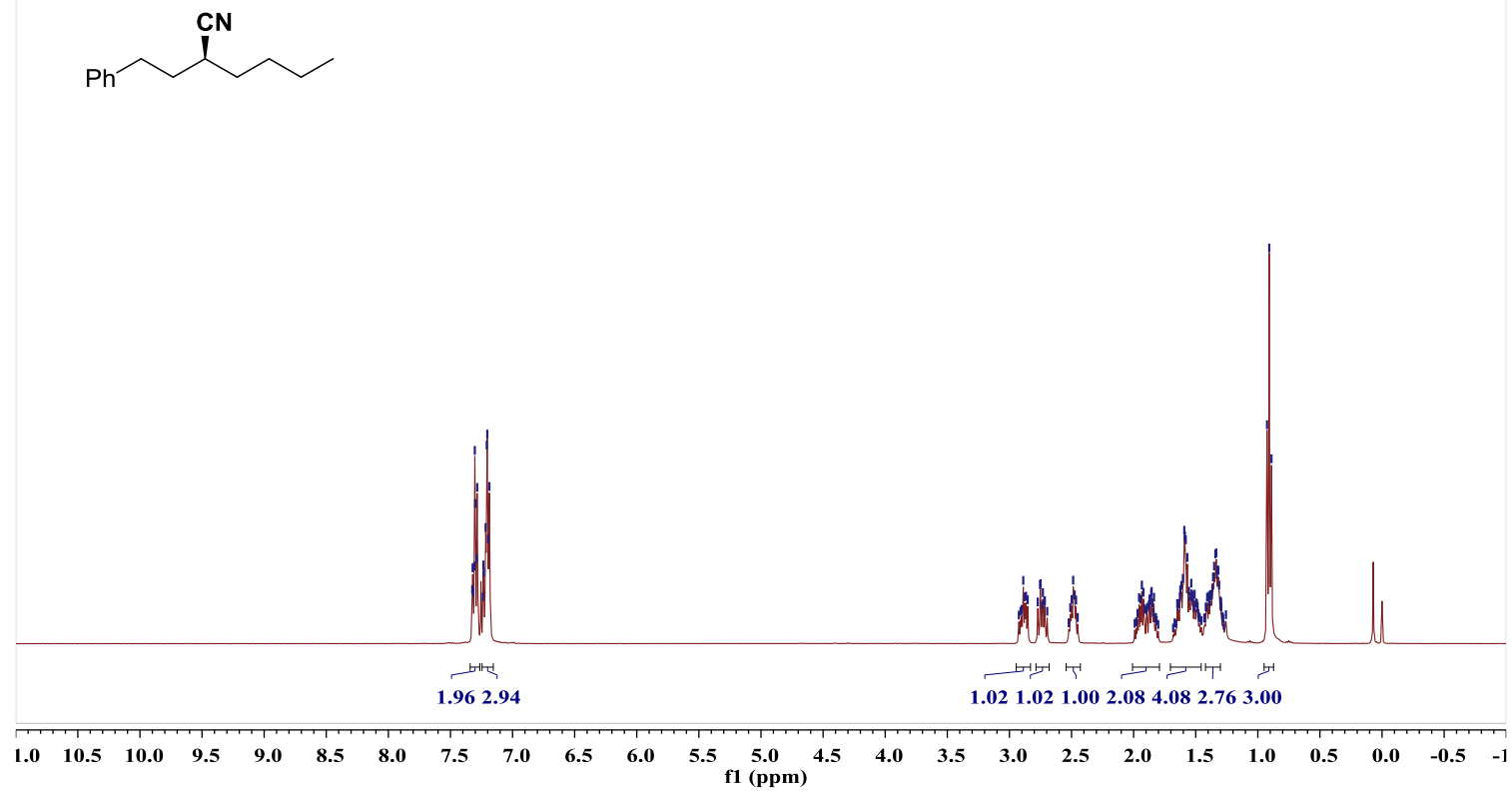

${ }^{13} \mathrm{C}$ NMR spectrum of compound $5 \mathrm{a}\left(100 \mathrm{MHz}, \mathrm{CDCl}_{3}\right)$

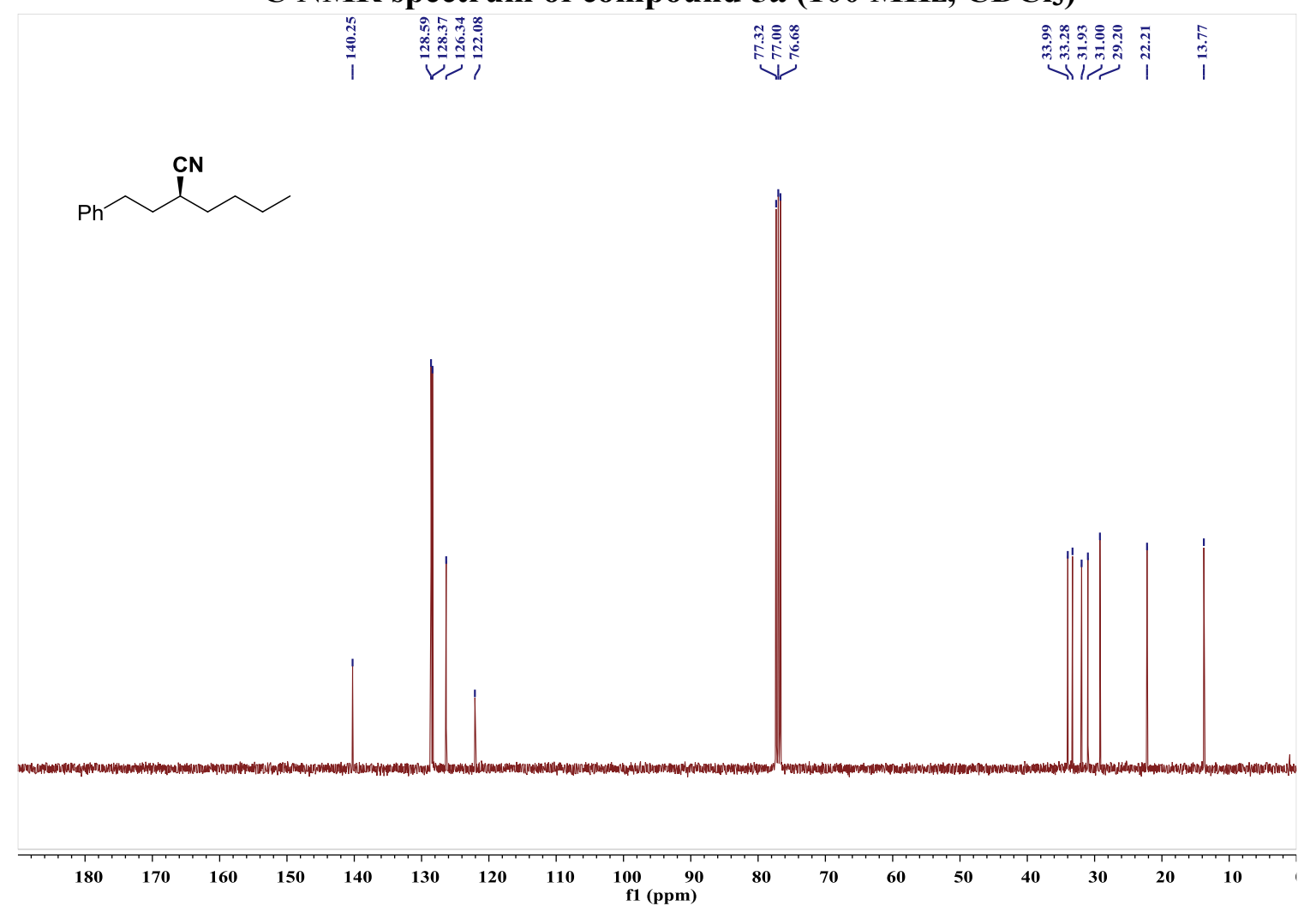


${ }^{1} \mathrm{H}$ NMR spectrum of compound $5 \mathrm{~b}\left(400 \mathrm{MHz}, \mathrm{CDCl}_{3}\right)$

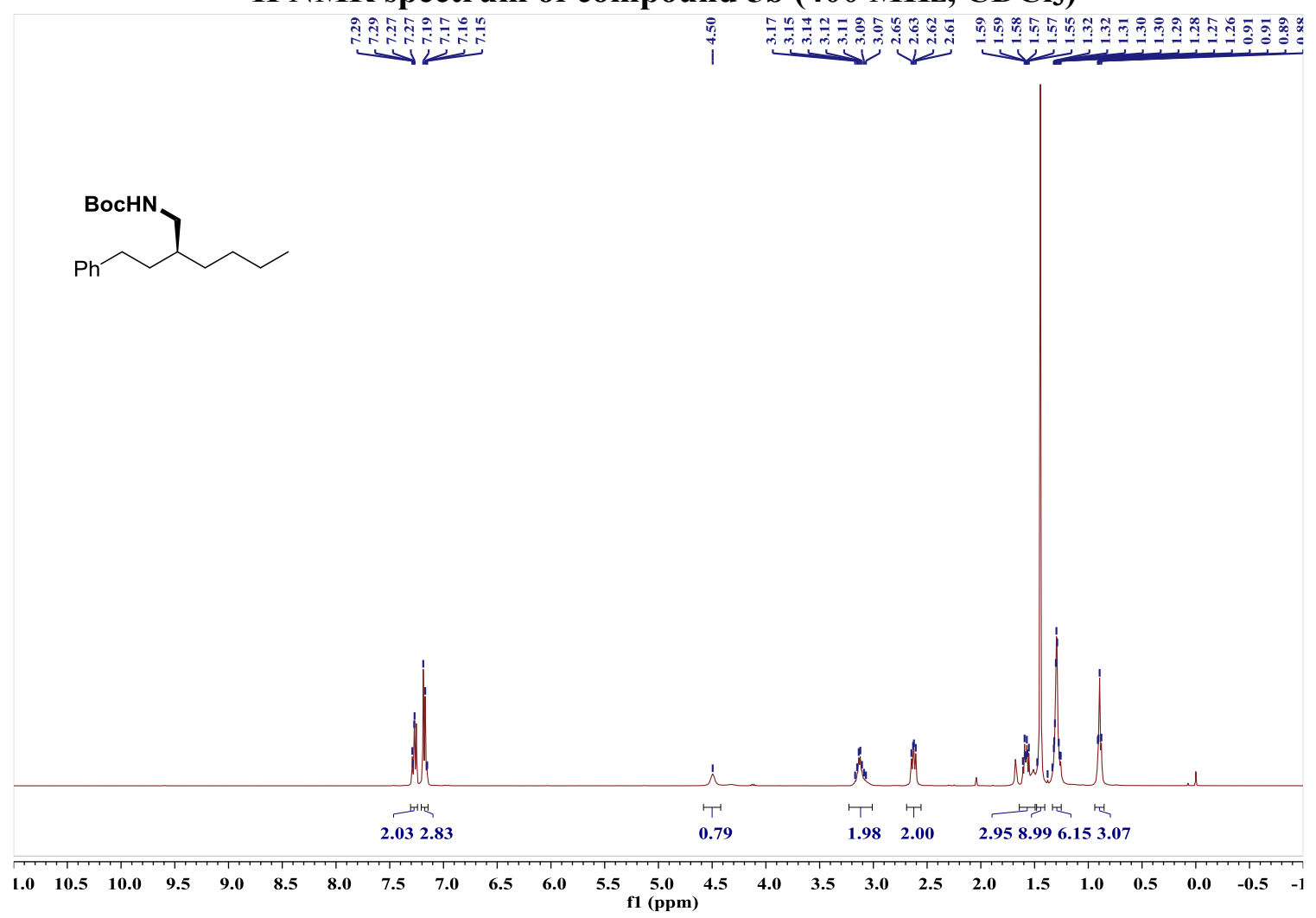

${ }^{13} \mathrm{C}$ NMR spectrum of compound $5 \mathrm{~b}\left(100 \mathrm{MHz}, \mathrm{CDCl}_{3}\right)$

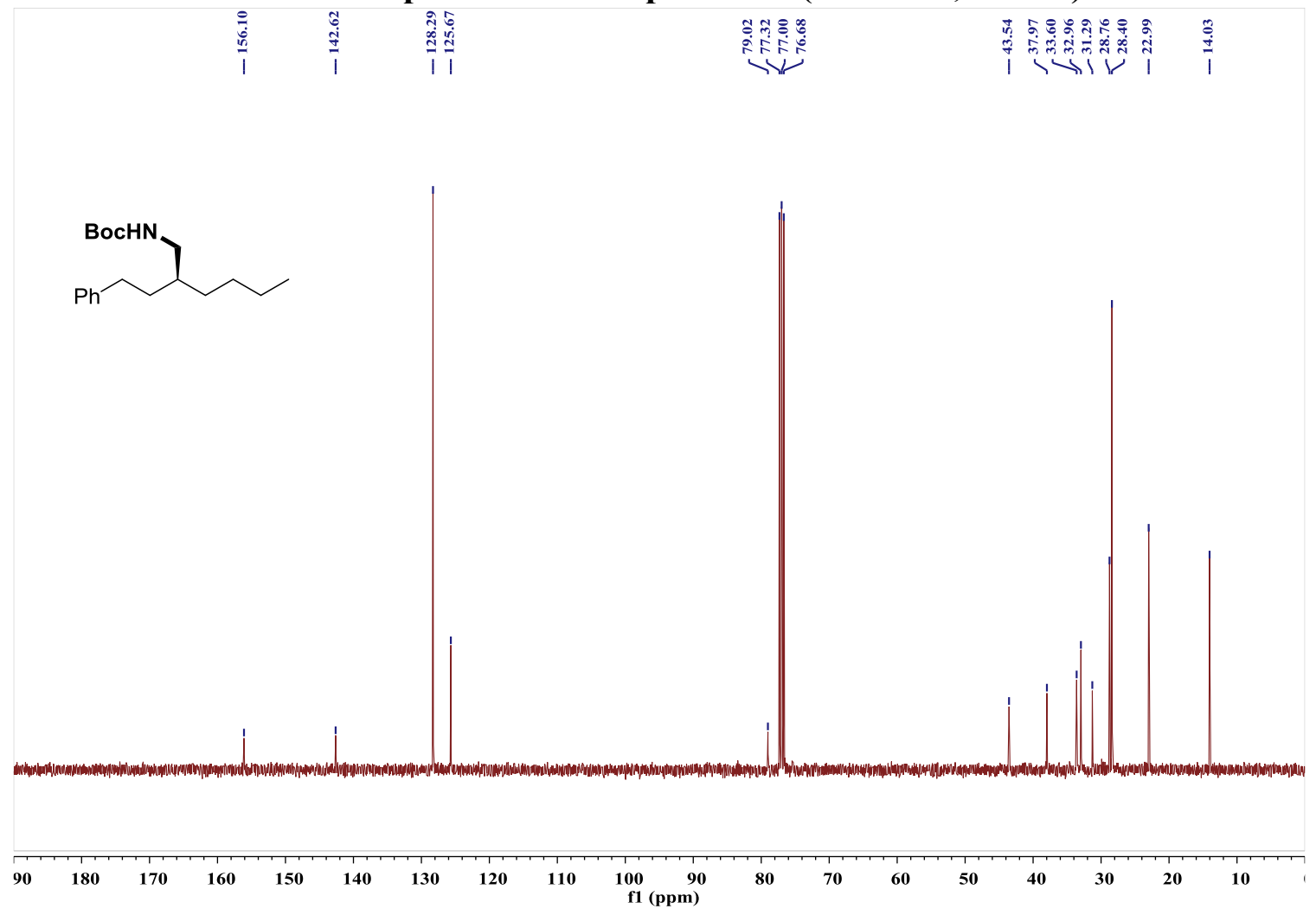

S61 
${ }^{1} \mathrm{H}$ NMR spectrum of compound $5 \mathrm{c}\left(400 \mathrm{MHz}, \mathrm{CDCl}_{3}\right)$

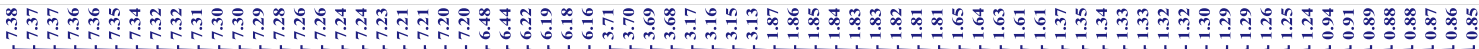<smiles>CCCCC(/C=C/c1ccccc1)C(C)=O</smiles>

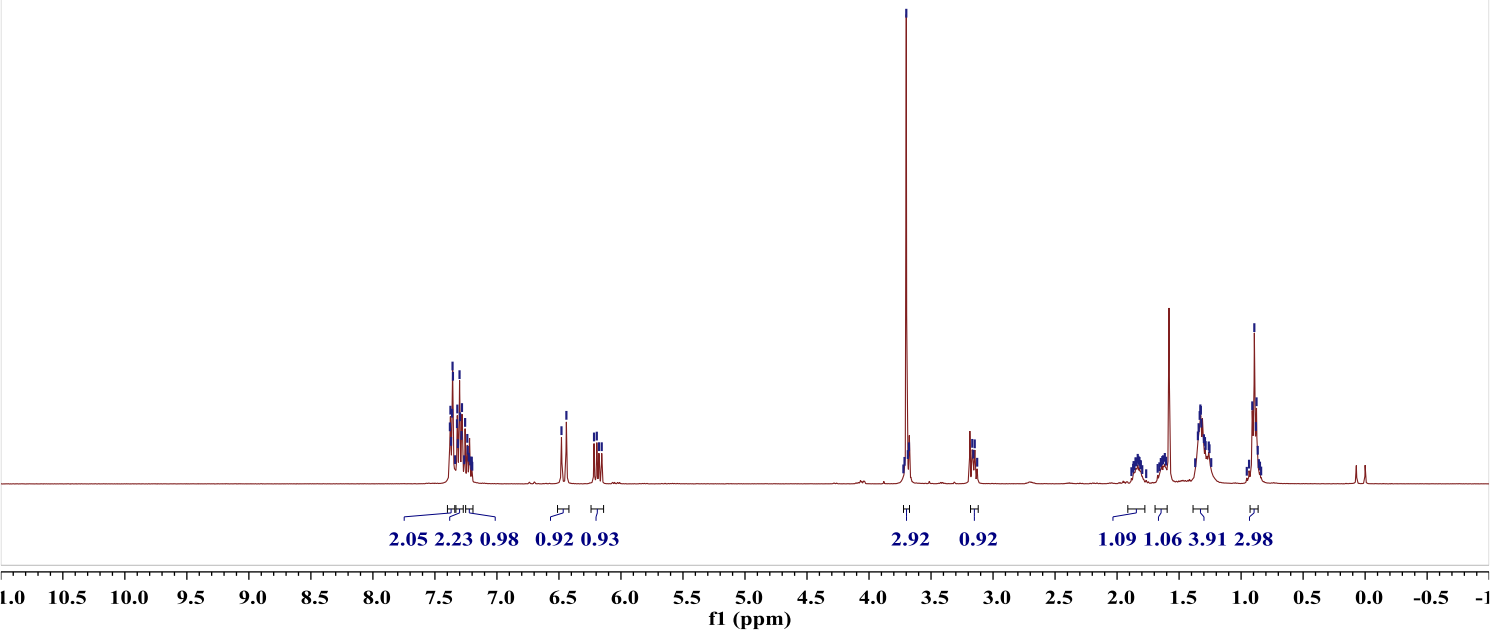

${ }^{13} \mathrm{C}$ NMR spectrum of compound $5 \mathrm{c}\left(100 \mathrm{MHz}, \mathrm{CDCl}_{3}\right)$

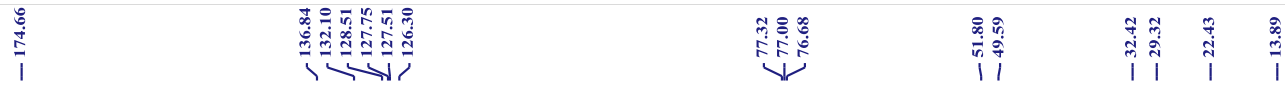
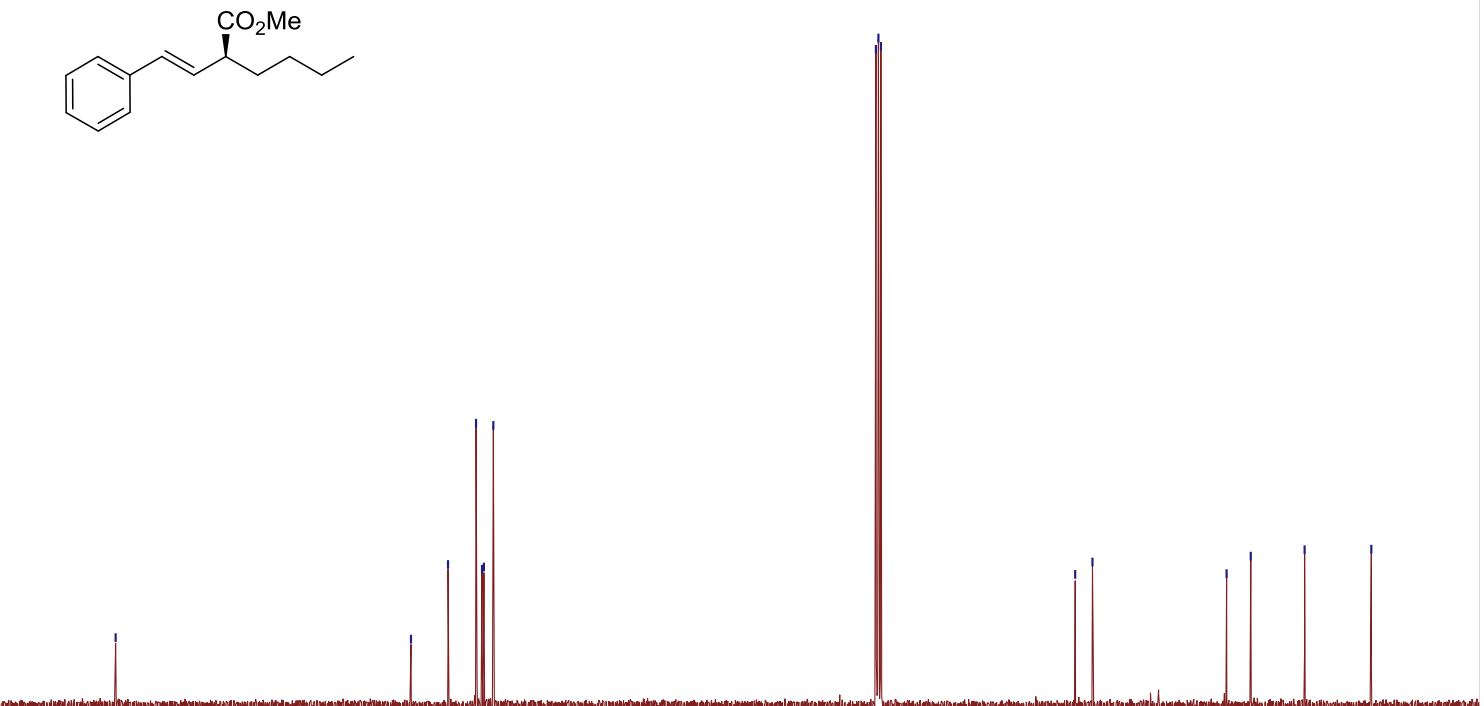

90

$\begin{array}{llllllllll}180 & 170 & 160 & 150 & 140 & 130 & 120 & 110 & \begin{array}{c}100 \\ \text { f1 (ppm) }\end{array}\end{array}$ 
${ }^{1} \mathrm{H}$ NMR spectrum of compound $5 \mathrm{~d}\left(400 \mathrm{MHz}, \mathrm{CDCl}_{3}\right)$

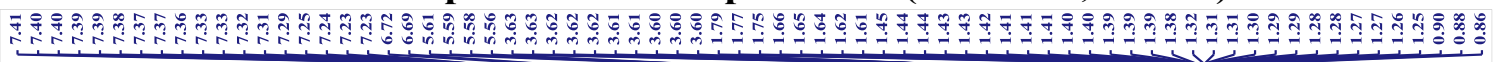
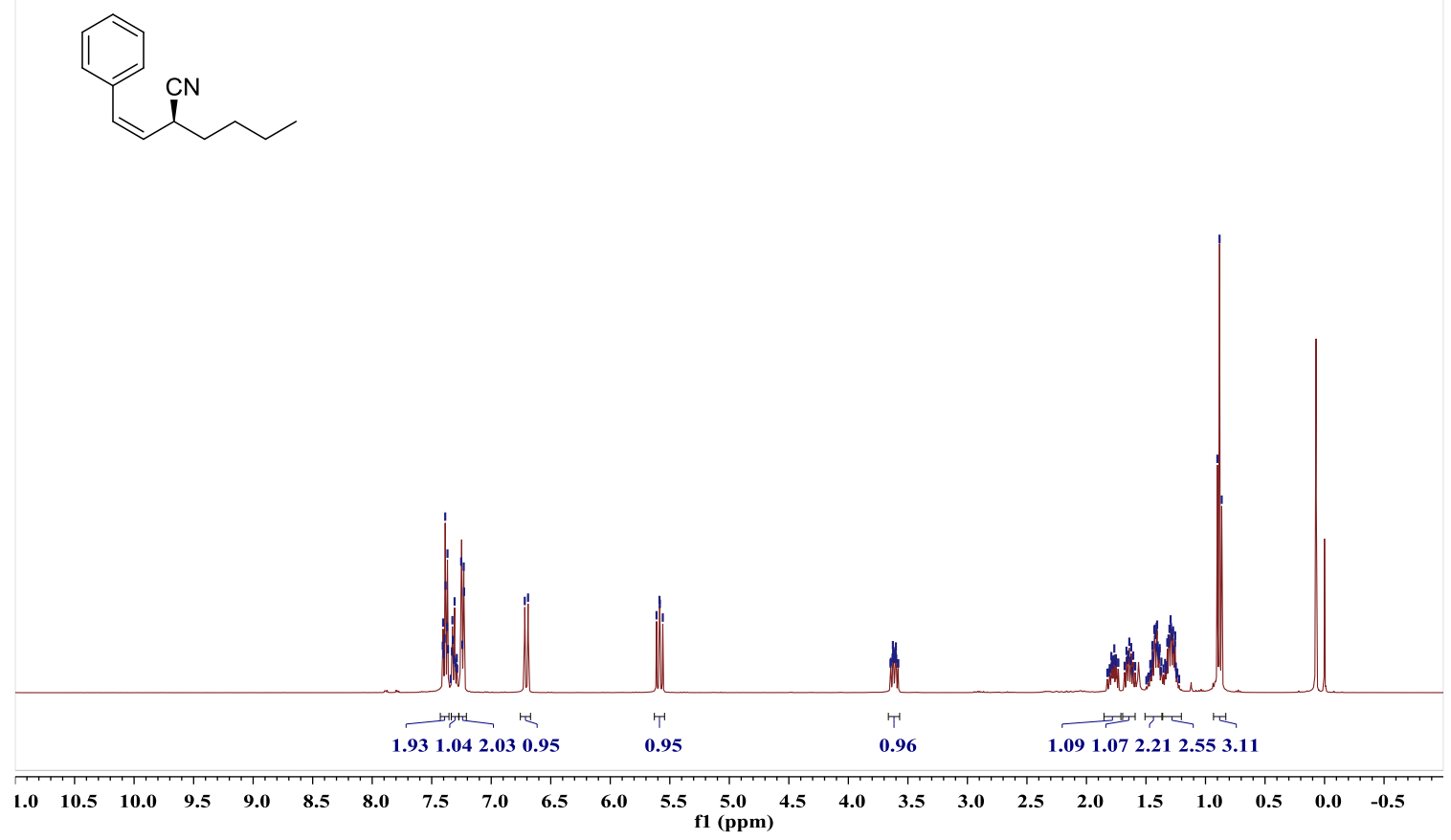

${ }^{13} \mathrm{C}$ NMR spectrum of compound $5 \mathrm{~d}\left(100 \mathrm{MHz}, \mathrm{CDCl}_{3}\right)$

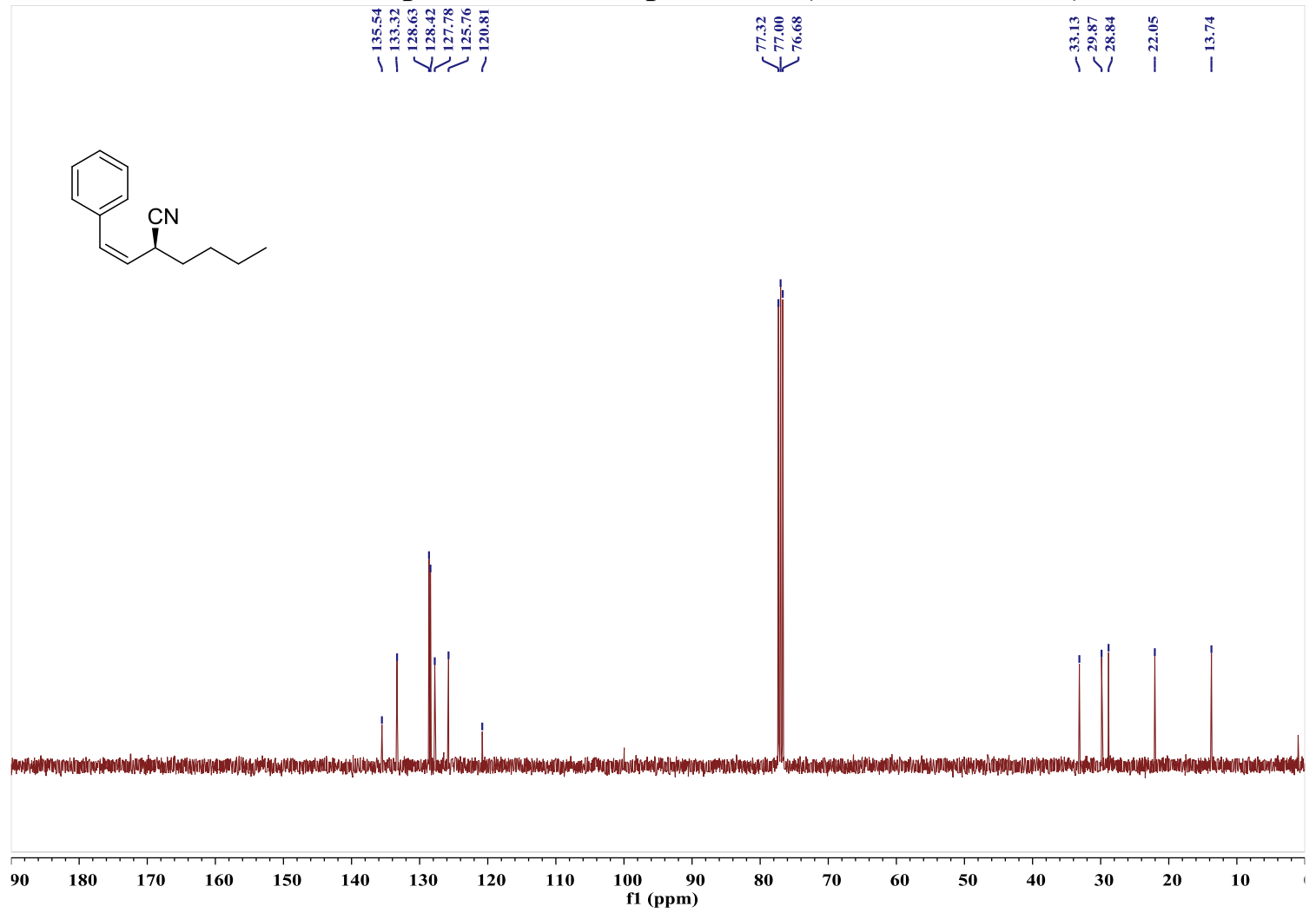

S63 
${ }^{1} \mathrm{H}$ NMR spectrum of compound $6 \mathrm{a}\left(400 \mathrm{MHz}, \mathrm{CDCl}_{3}\right)$

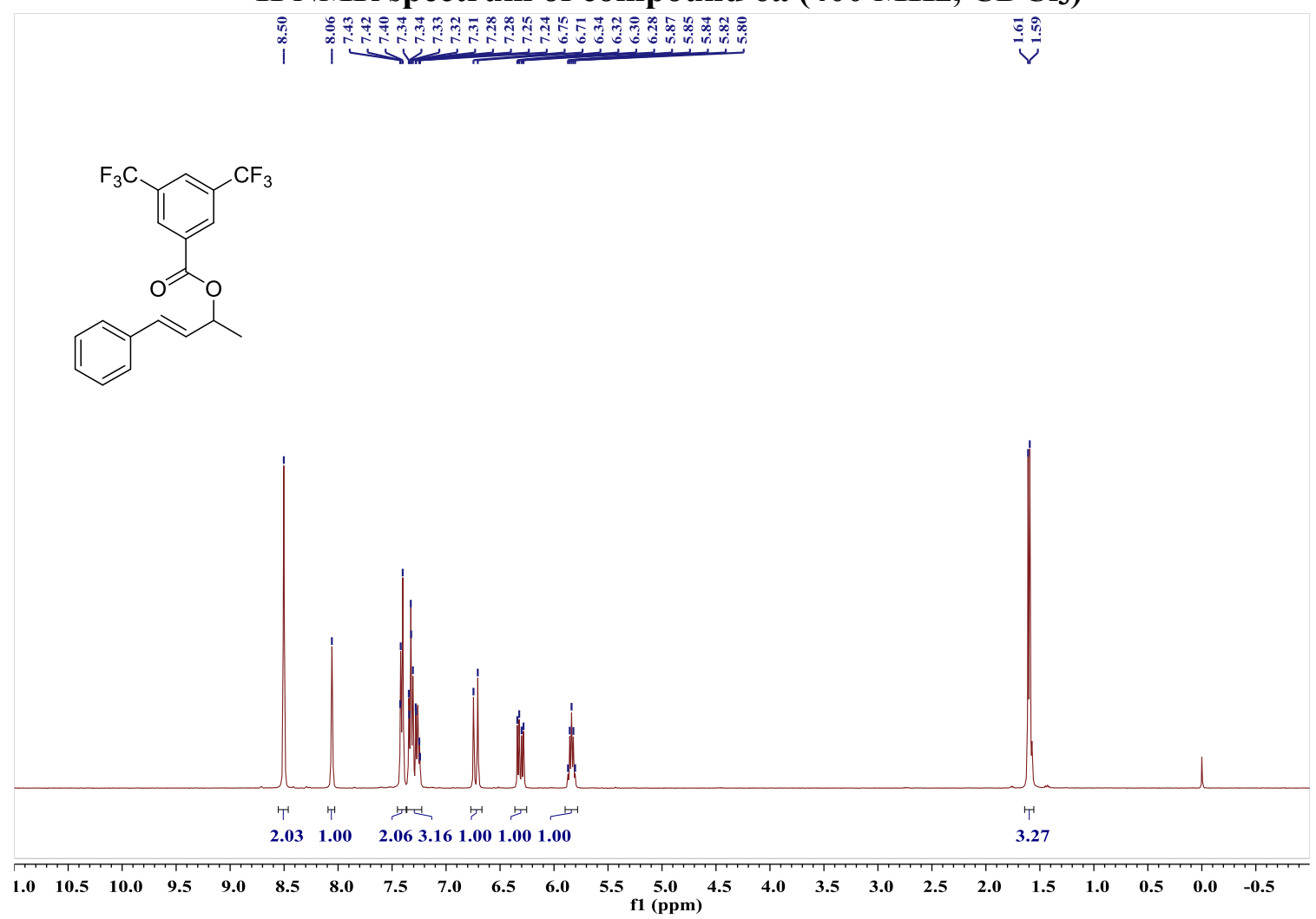

${ }^{13} \mathrm{C}$ NMR spectrum of compound $6 \mathrm{a}\left(100 \mathrm{MHz}, \mathrm{CDCl}_{3}\right)$

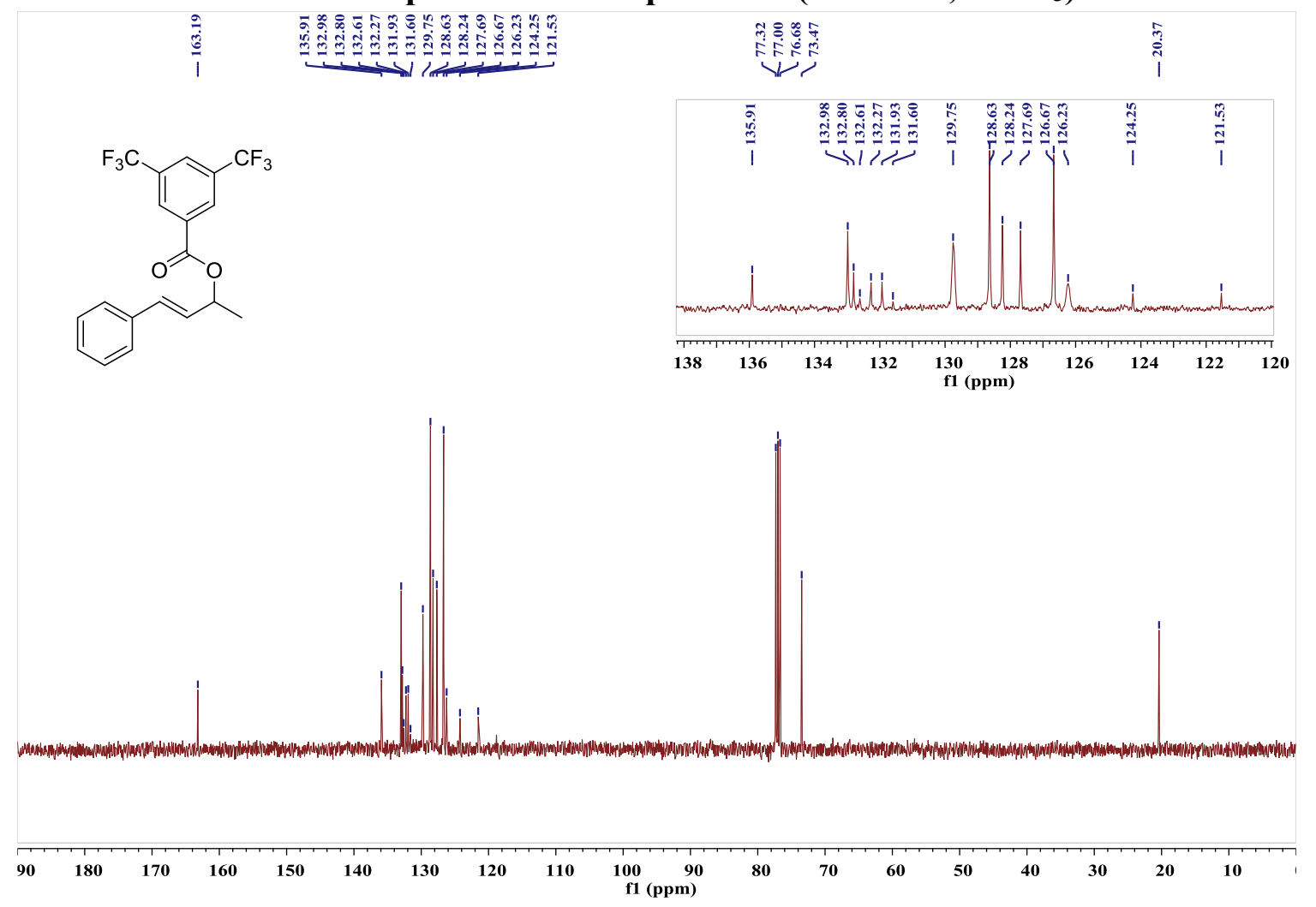


${ }^{19} \mathrm{~F}$ NMR spectrum of compound $6 \mathrm{a}\left(376 \mathrm{MHz}, \mathrm{CDCl}_{3}\right)$

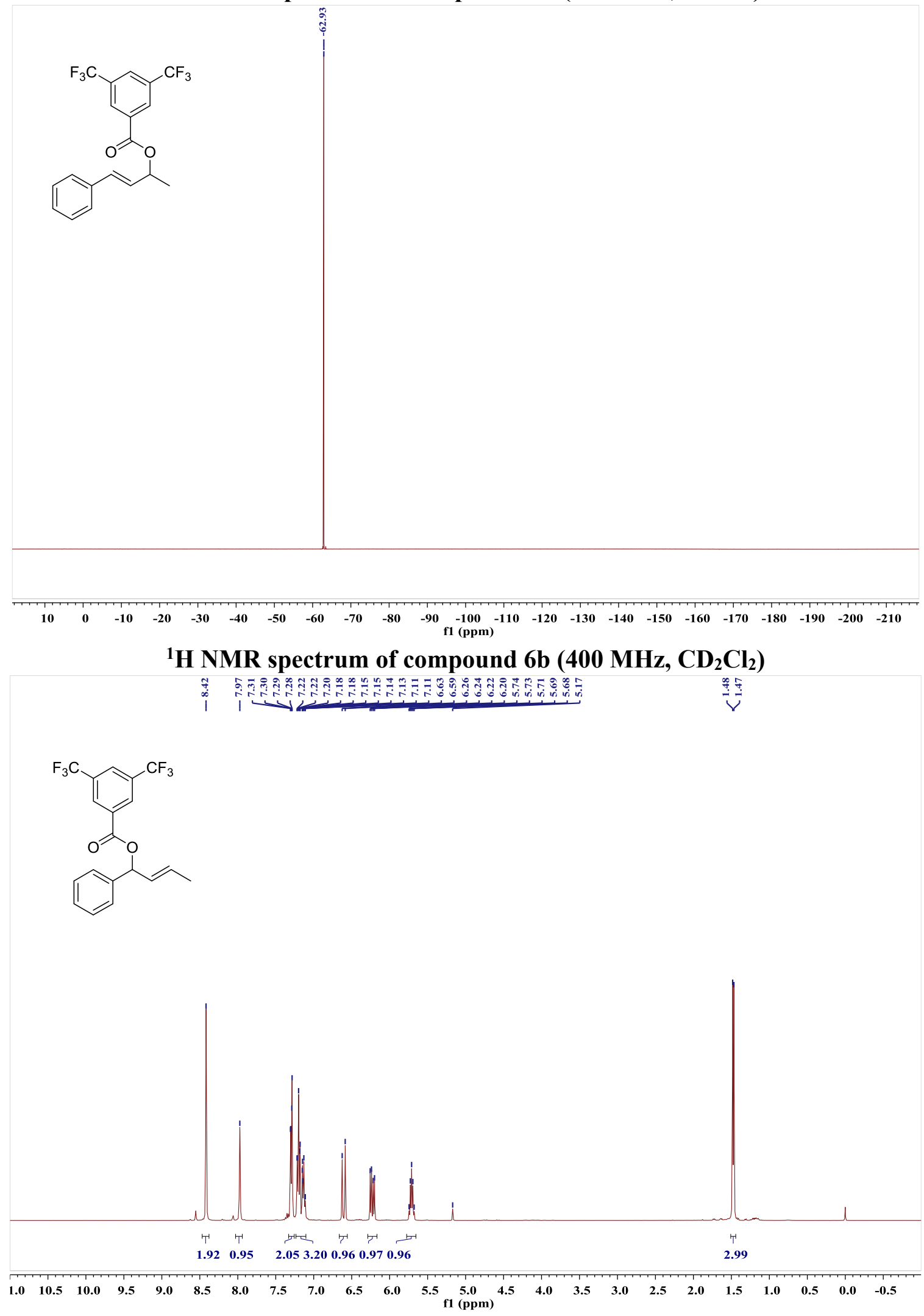



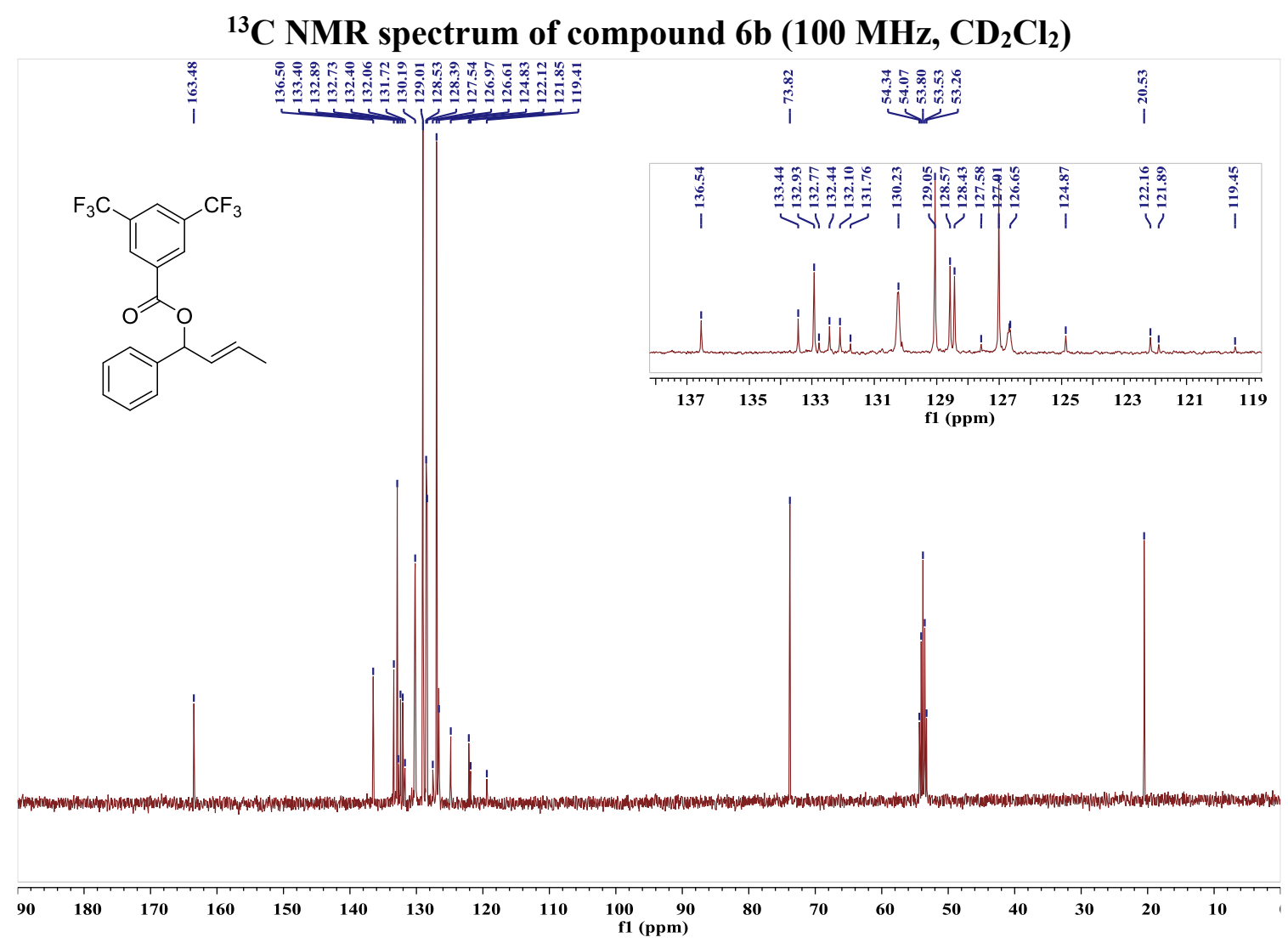

${ }^{19} \mathrm{~F}$ NMR spectrum of compound $6 \mathrm{~b}\left(376 \mathrm{MHz}, \mathrm{CD}_{2} \mathrm{Cl}_{2}\right)$

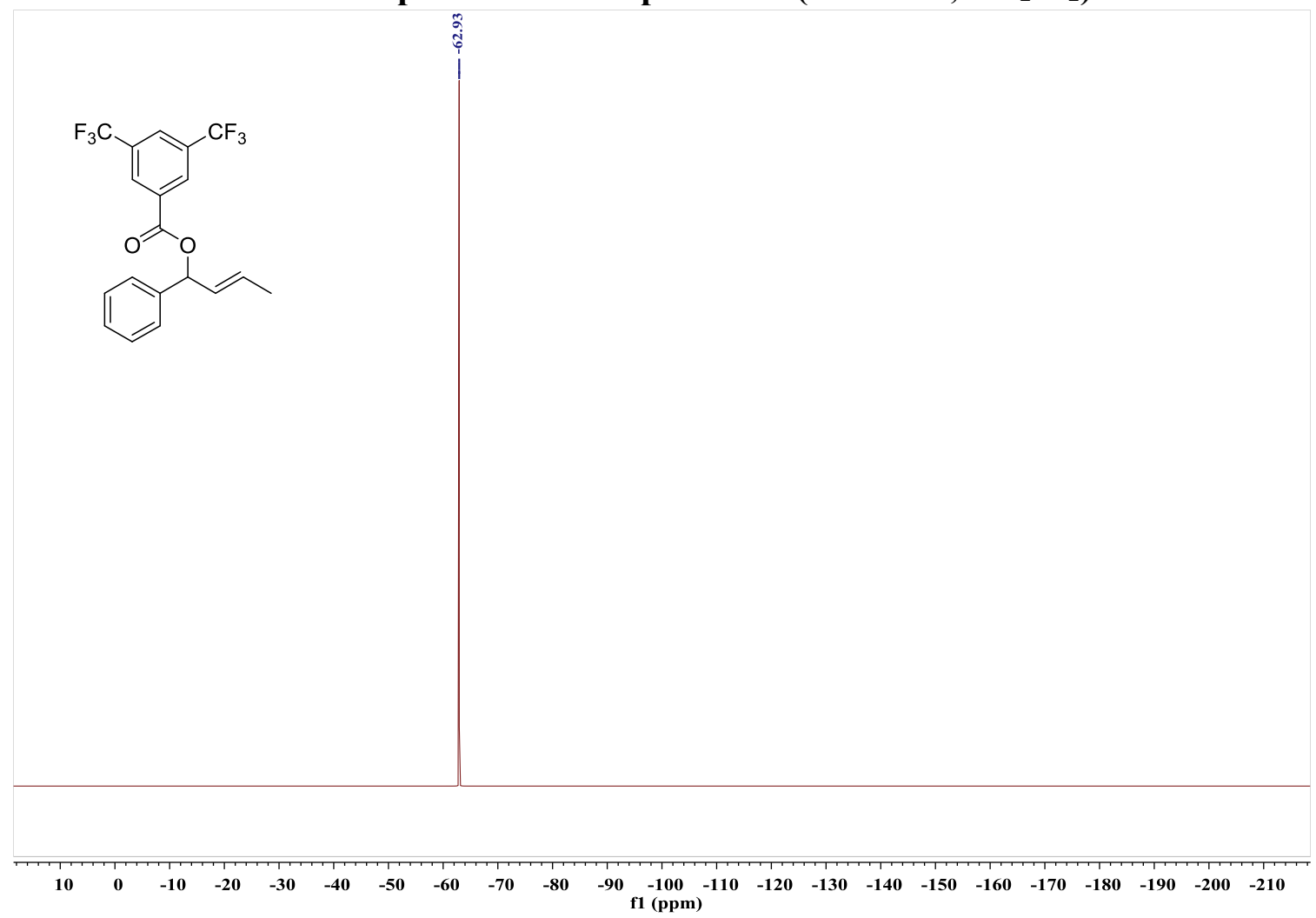


${ }^{1} \mathrm{H}$ NMR spectrum of compound $7\left(400 \mathrm{MHz}, \mathrm{CDCl}_{3}\right)$

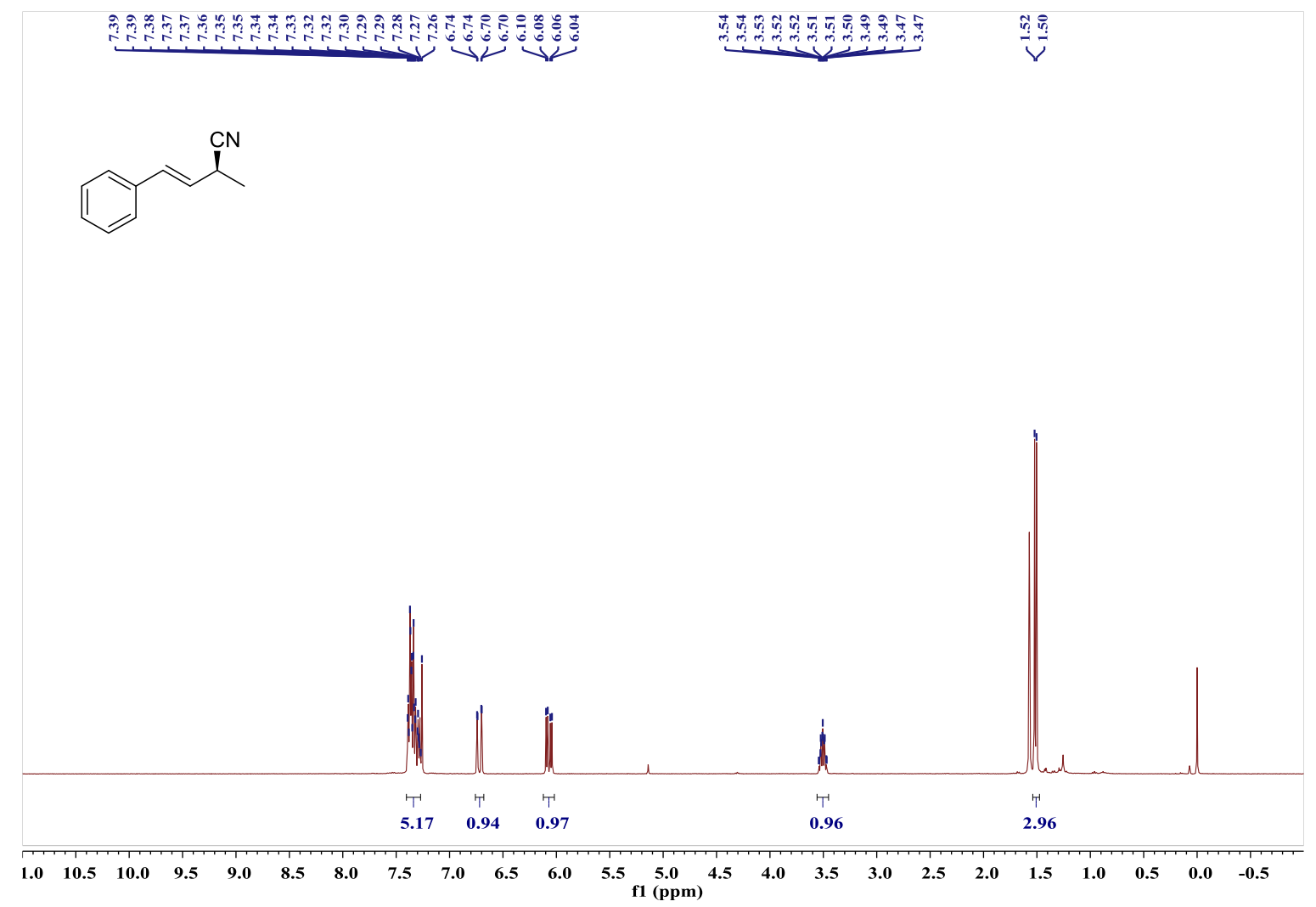

${ }^{13} \mathrm{C}$ NMR spectrum of compound $7\left(100 \mathrm{MHz}, \mathrm{CDCl}_{3}\right)$

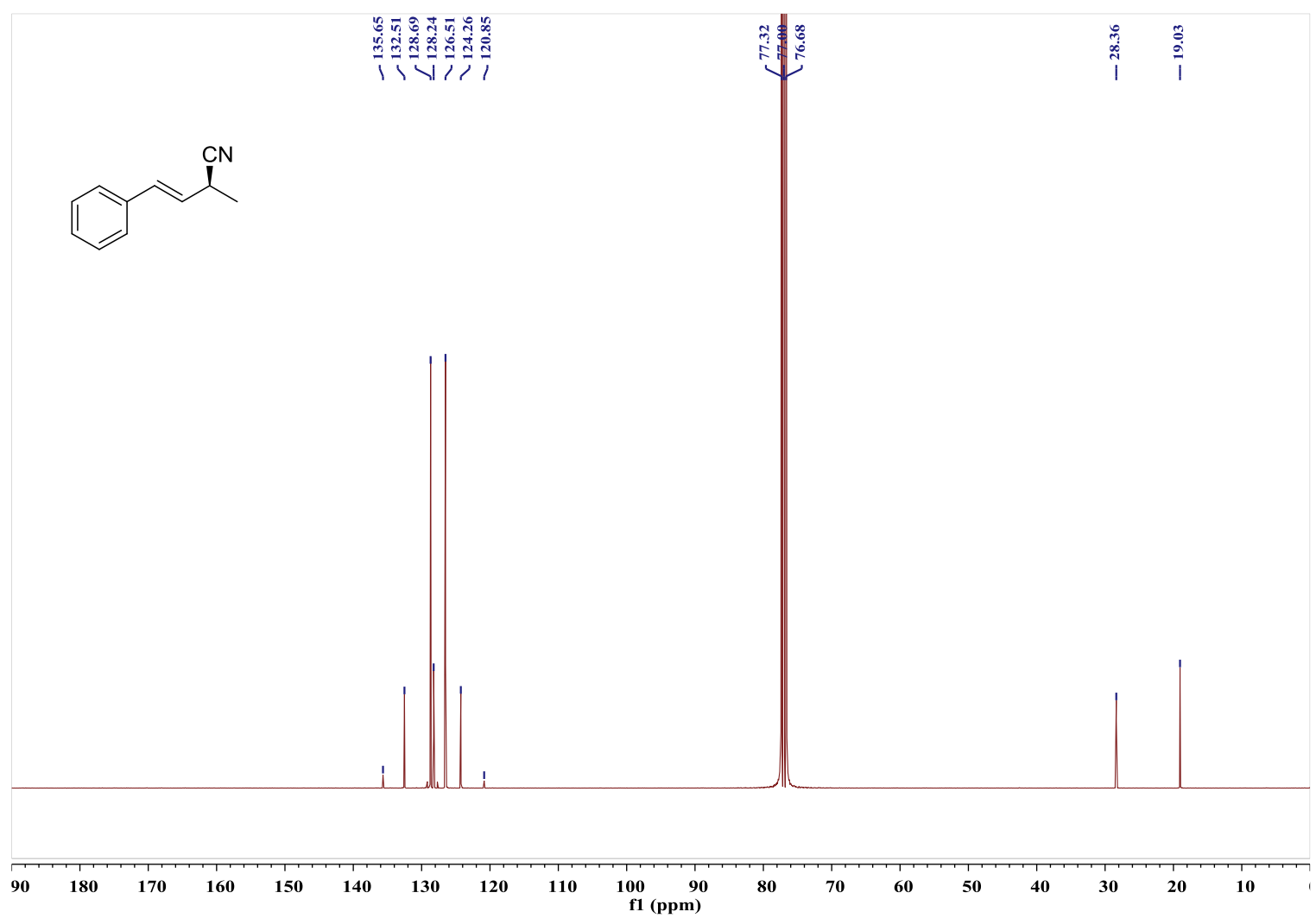


${ }^{1} \mathrm{H}$ NMR spectrum of compound 9a (400 $\left.\mathrm{MHz}, \mathrm{CDCl}_{3}\right)$

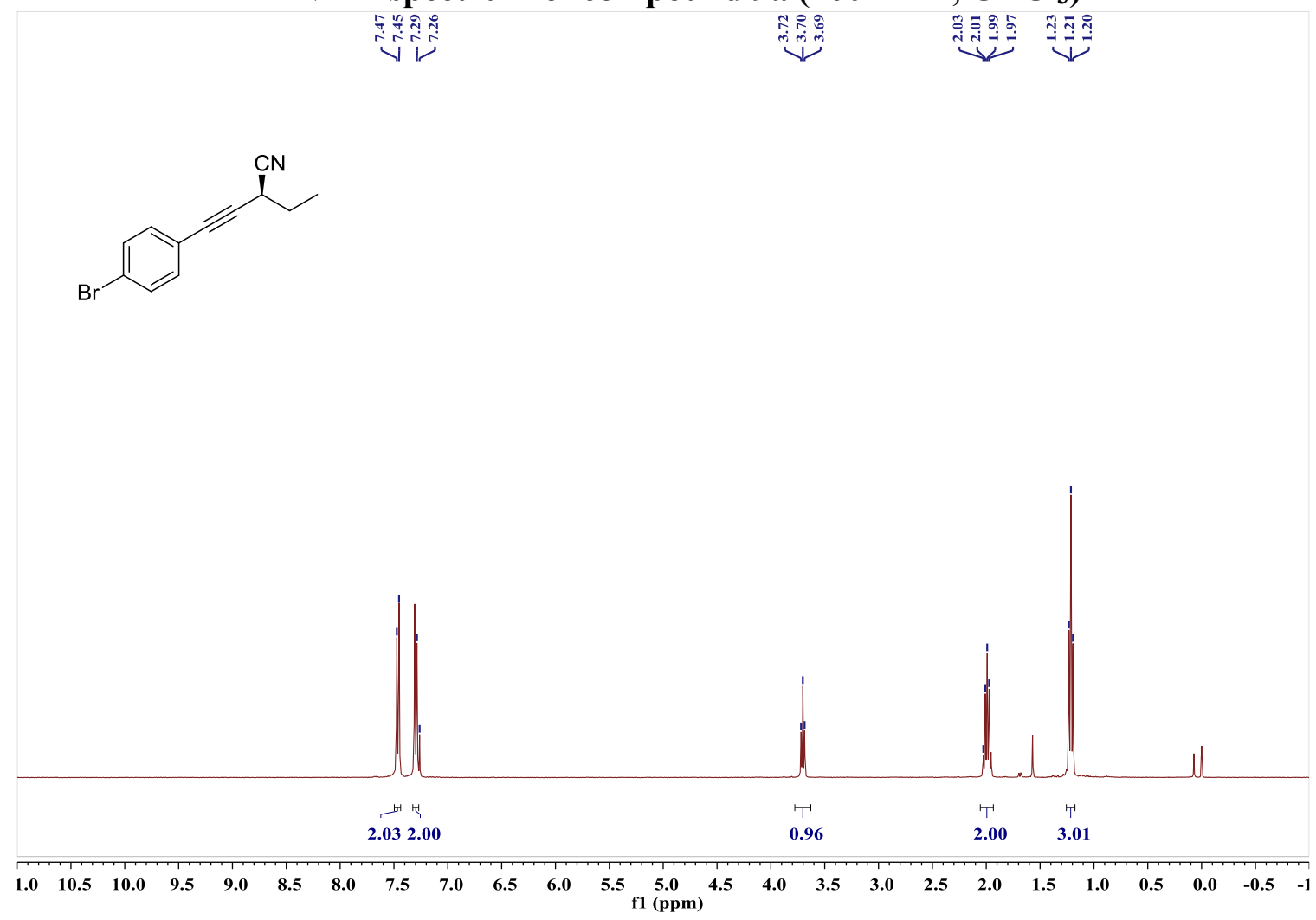

${ }^{13} \mathrm{C}$ NMR spectrum of compound 9a (100 $\left.\mathrm{MHz}, \mathrm{CDCl}_{3}\right)$

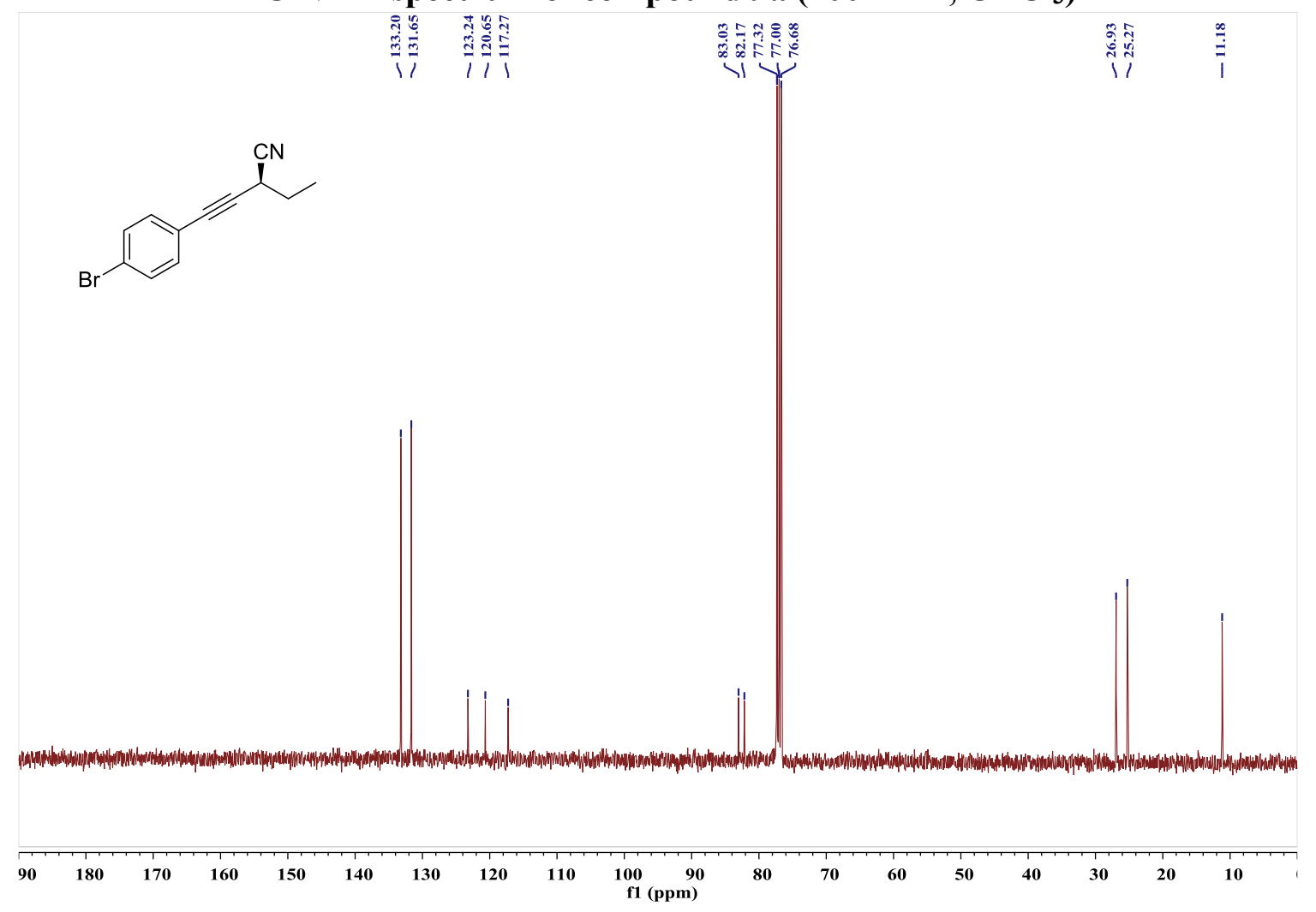


${ }^{1} \mathrm{H}$ NMR spectrum of compound $9 \mathrm{~b}\left(400 \mathrm{MHz}, \mathrm{CDCl}_{3}\right)$

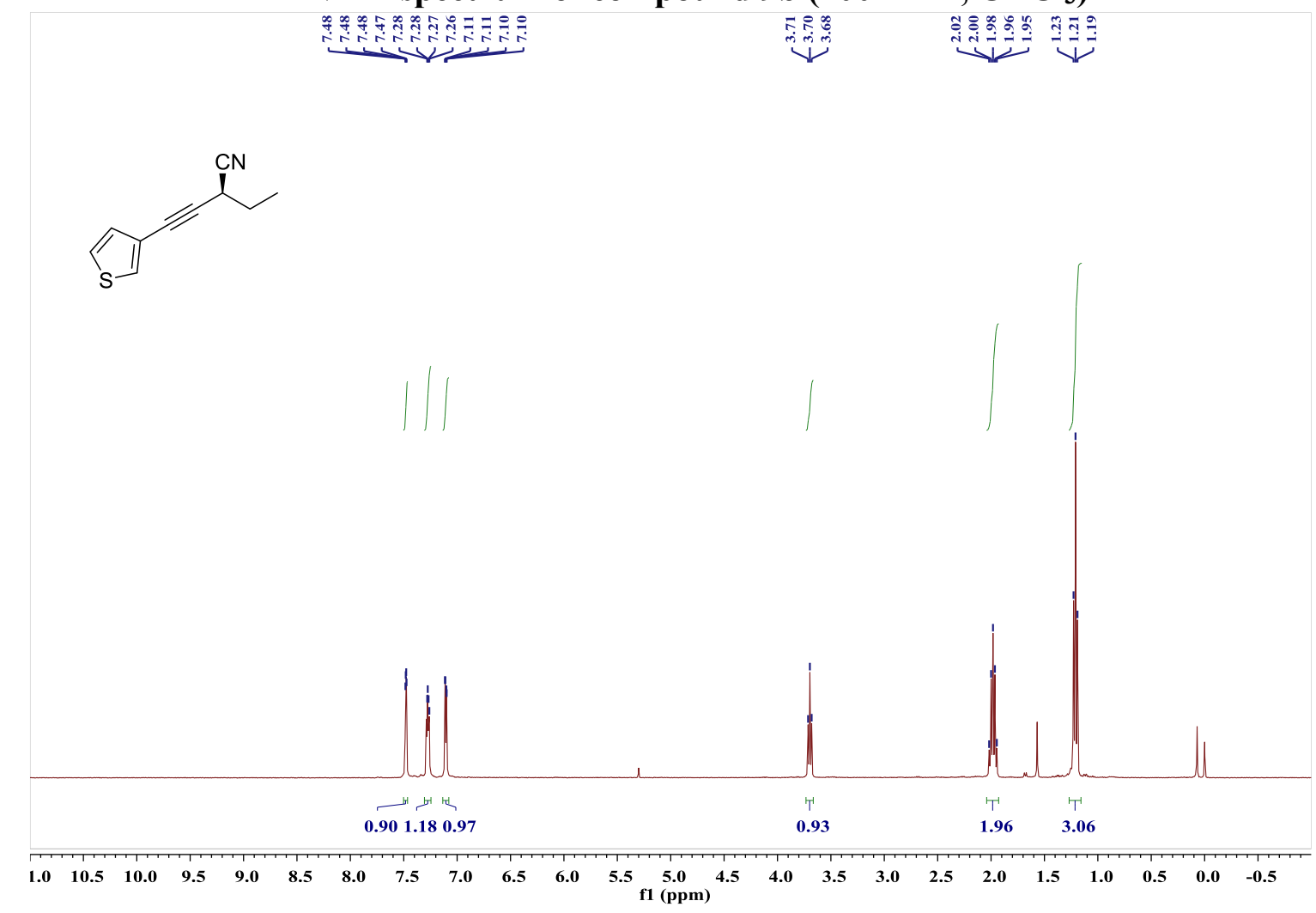

${ }^{13} \mathrm{C}$ NMR spectrum of compound $9 \mathrm{~b}\left(100 \mathrm{MHz}, \mathrm{CDCl}_{3}\right)$

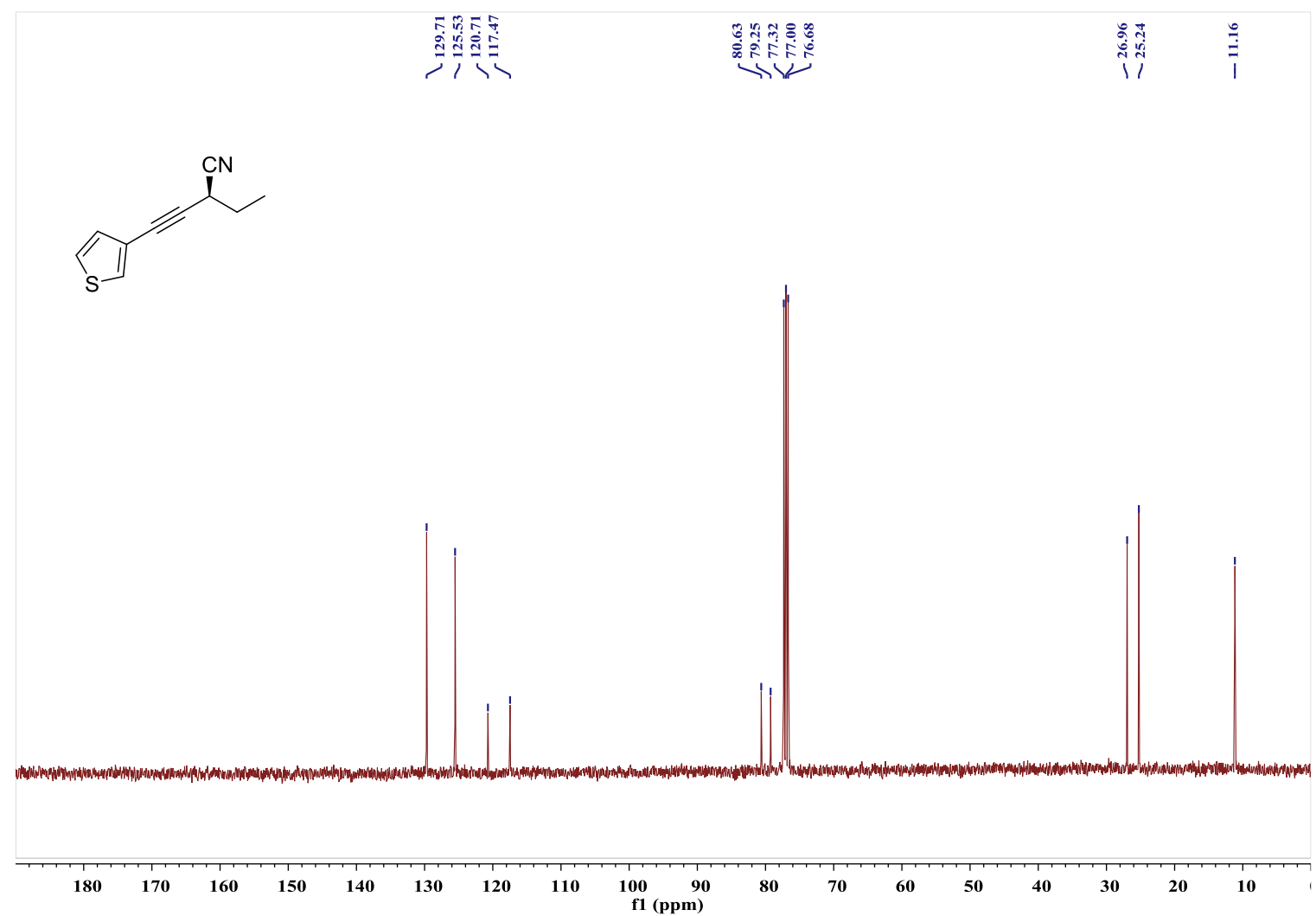


${ }^{1} \mathrm{H}$ NMR spectrum of compound $9 \mathrm{c}\left(400 \mathrm{MHz}, \mathrm{CDCl}_{3}\right)$

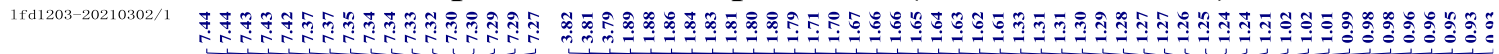
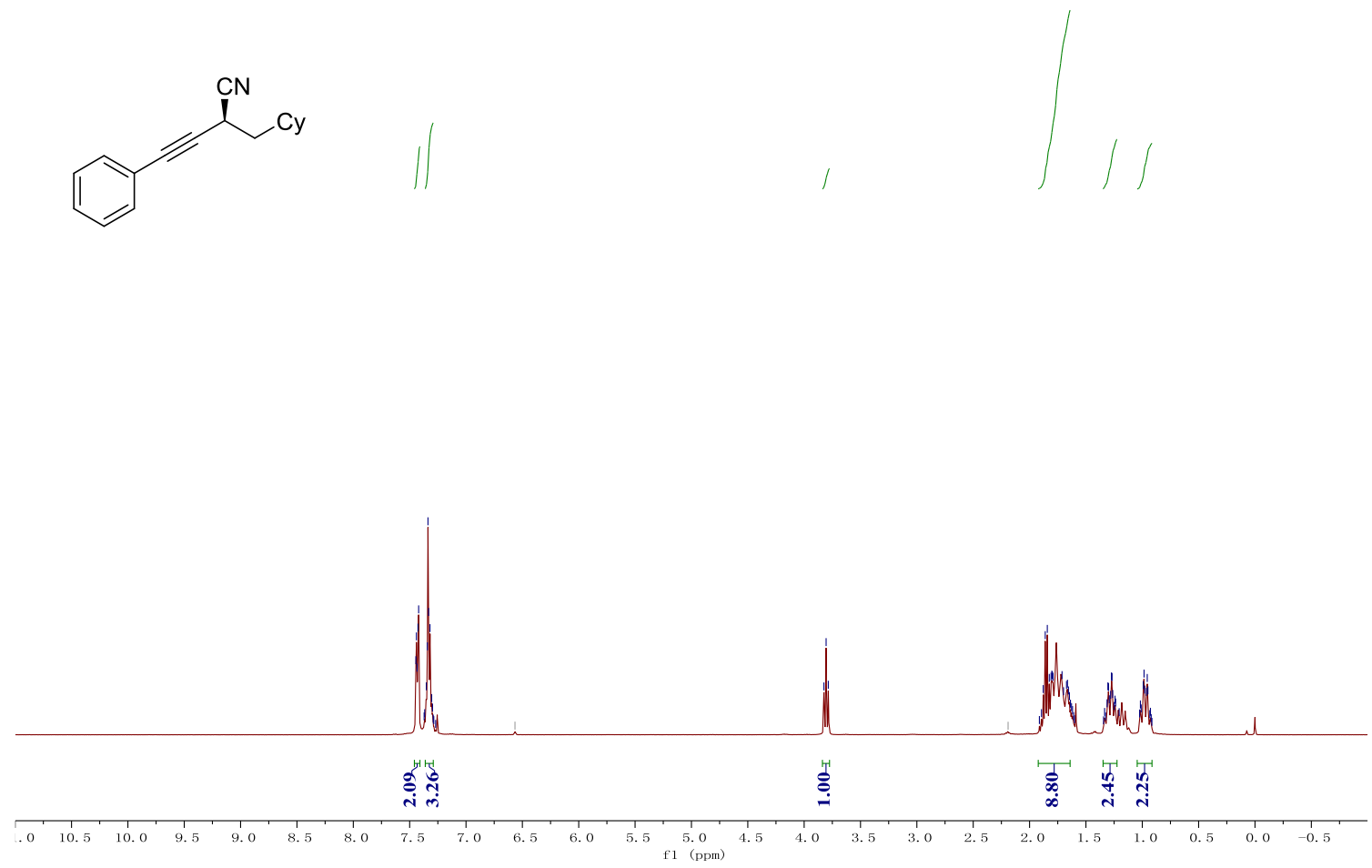

${ }^{13} \mathrm{C}$ NMR spectrum of compound 9c $\left(100 \mathrm{MHz}, \mathrm{CDCl}_{3}\right)$

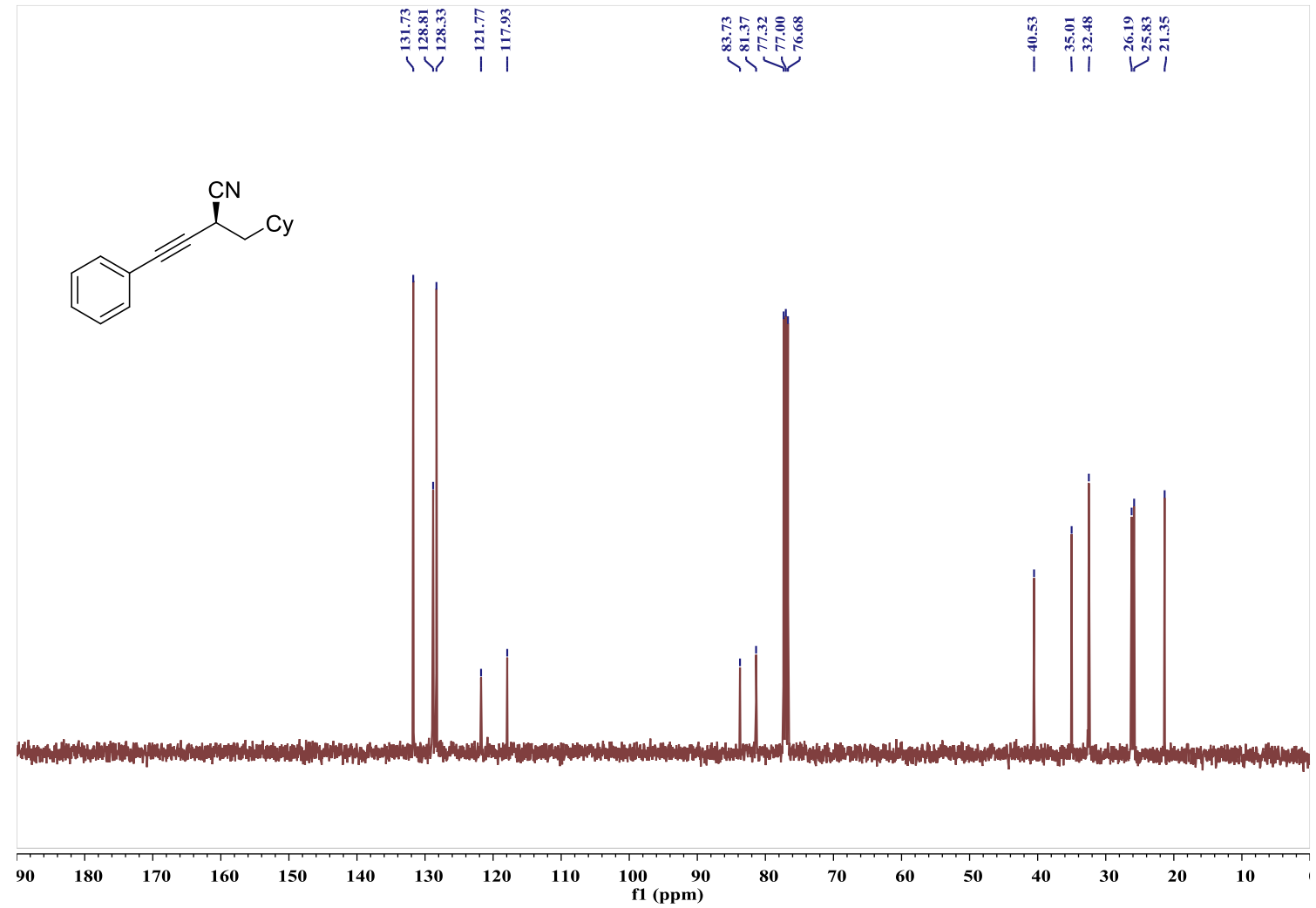


${ }^{1} \mathrm{H}$ NMR spectrum of compound $10\left(400 \mathrm{MHz}, \mathrm{CDCl}_{3}\right)$

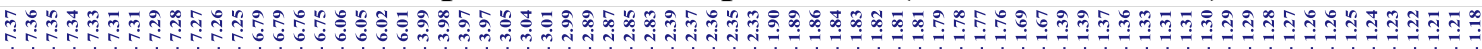

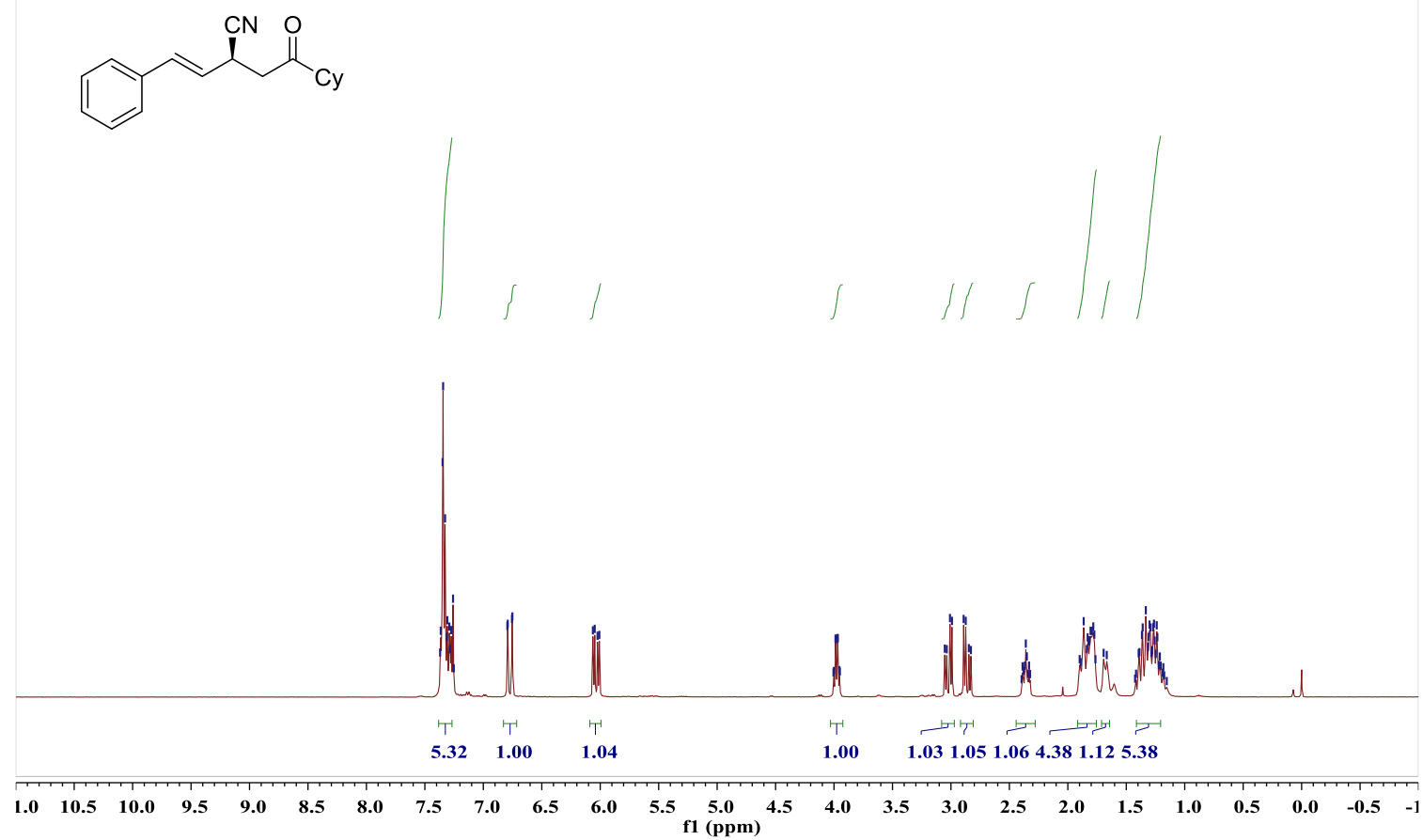

${ }^{13} \mathrm{C}$ NMR spectrum of compound $10\left(100 \mathrm{MHz}, \mathrm{CDCl}_{3}\right)$

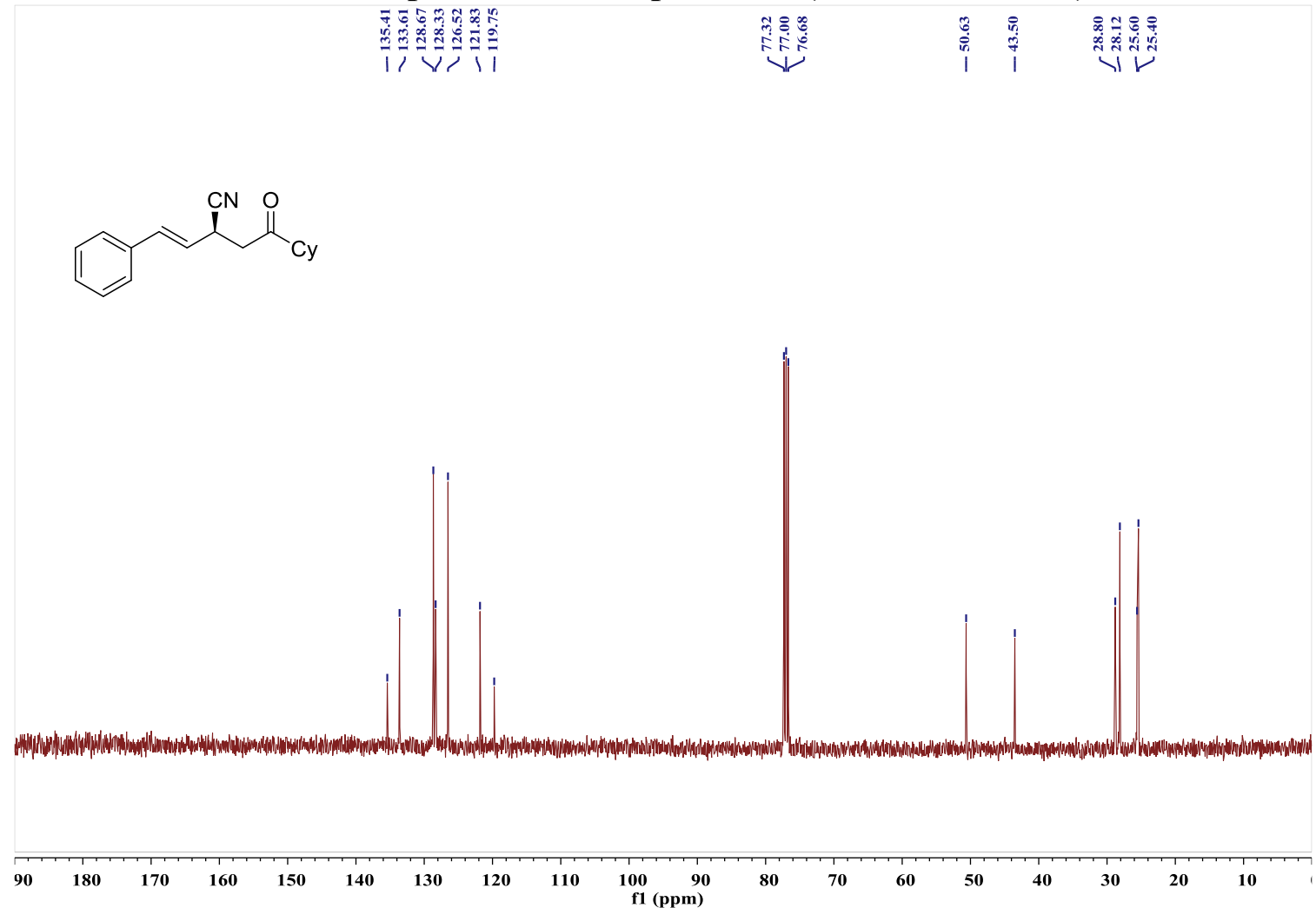




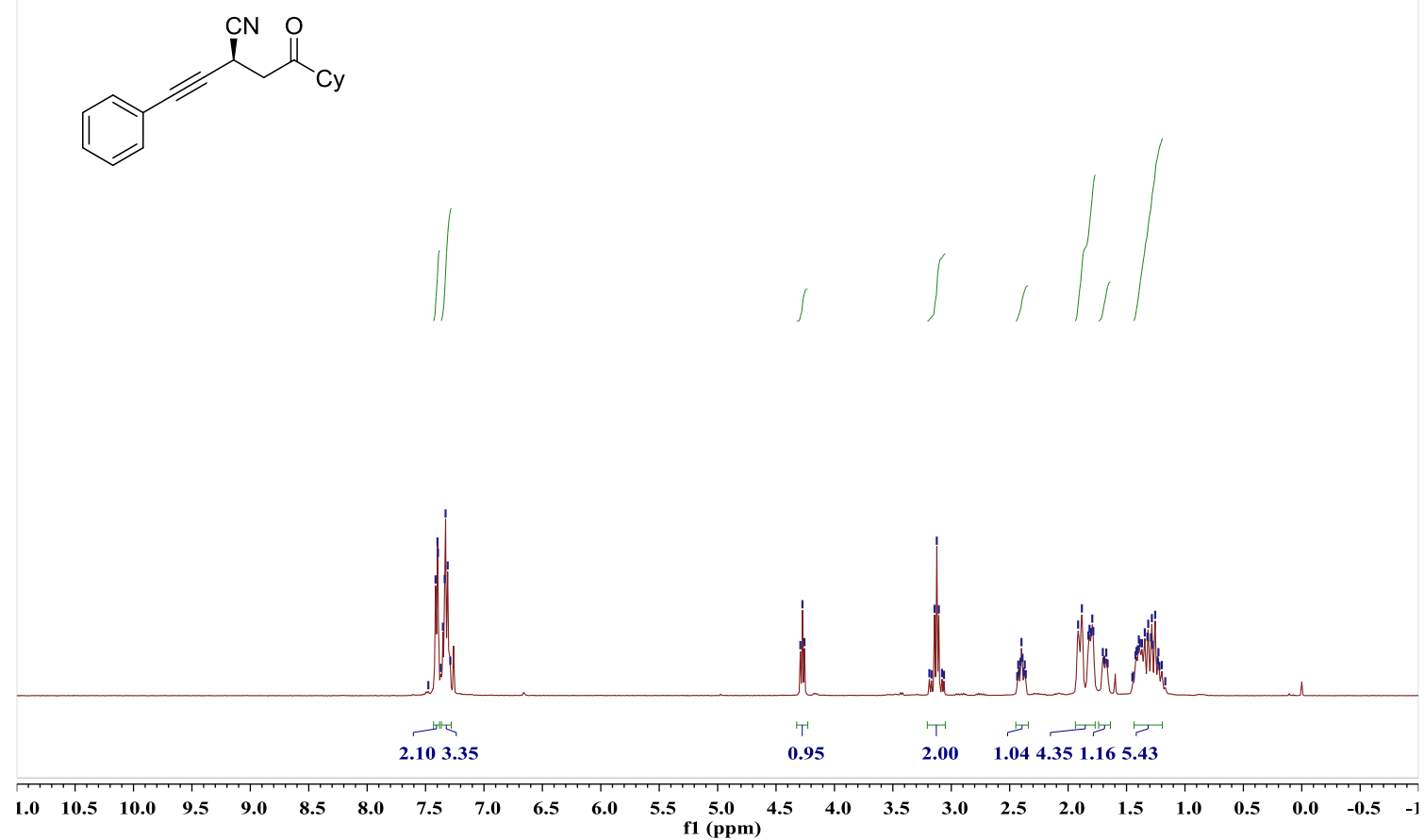

${ }^{13} \mathrm{C}$ NMR spectrum of compound $11\left(100 \mathrm{MHz}, \mathrm{CDCl}_{3}\right)$
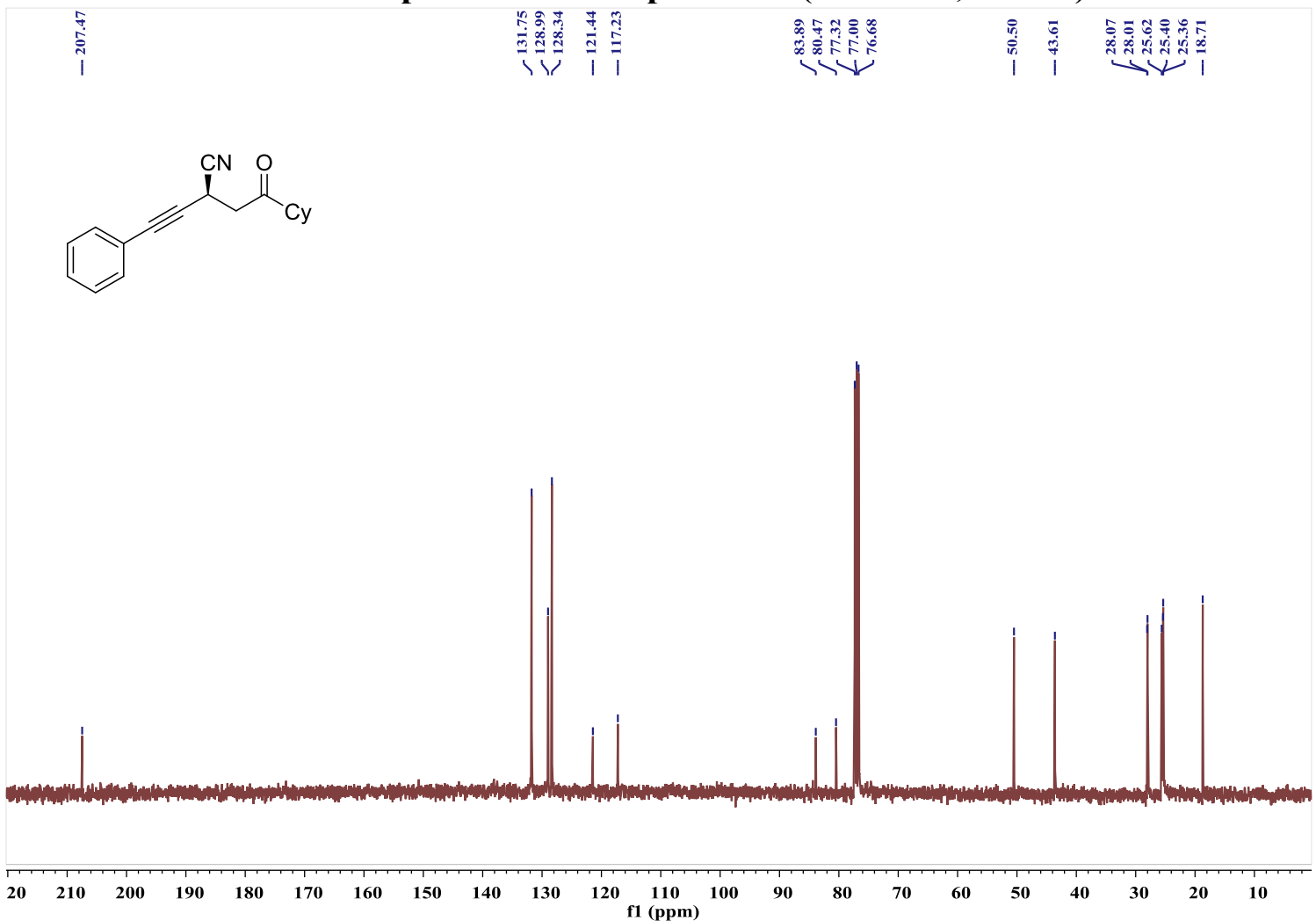


\section{Copies of HPLC Spectra}
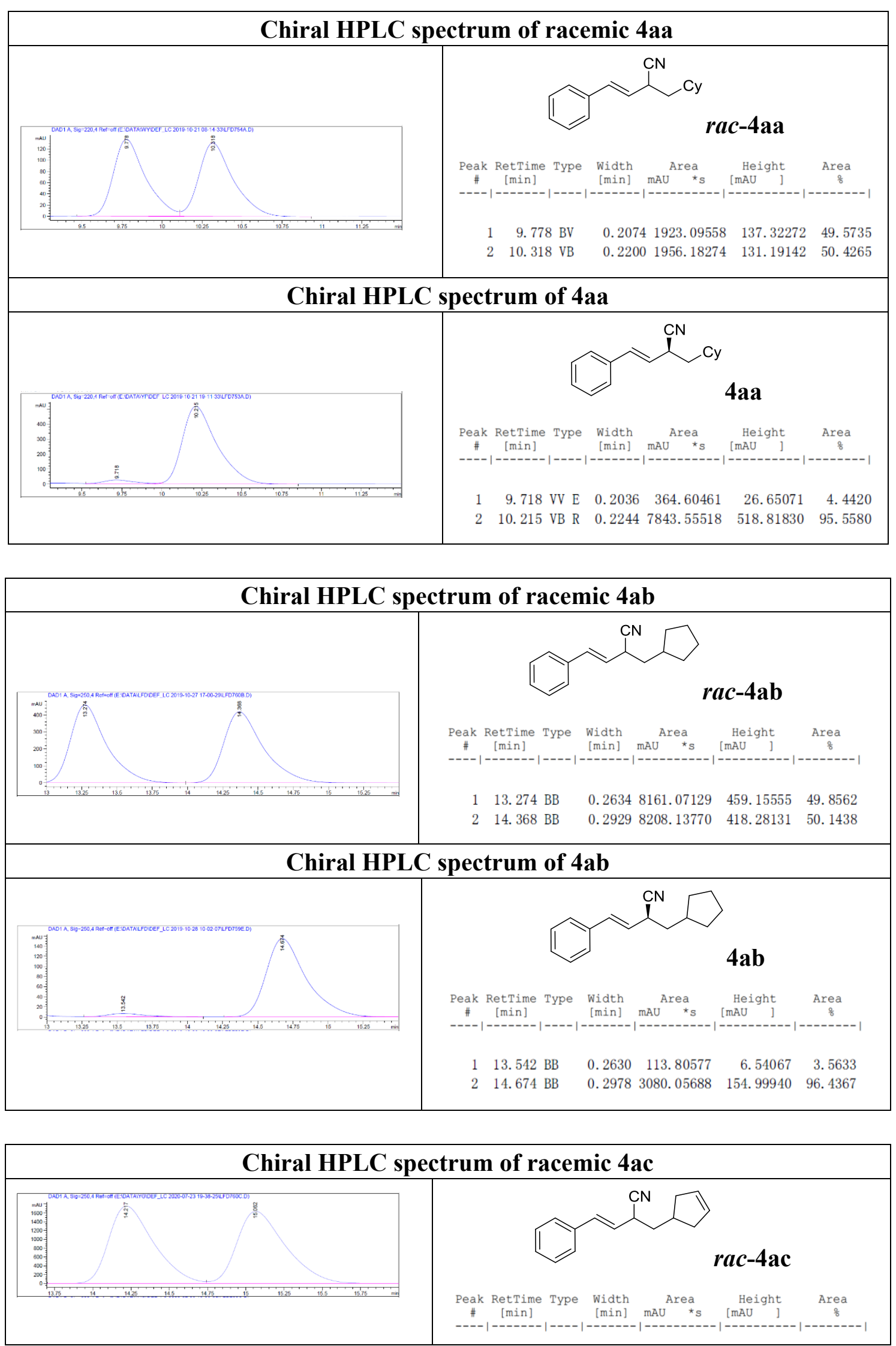

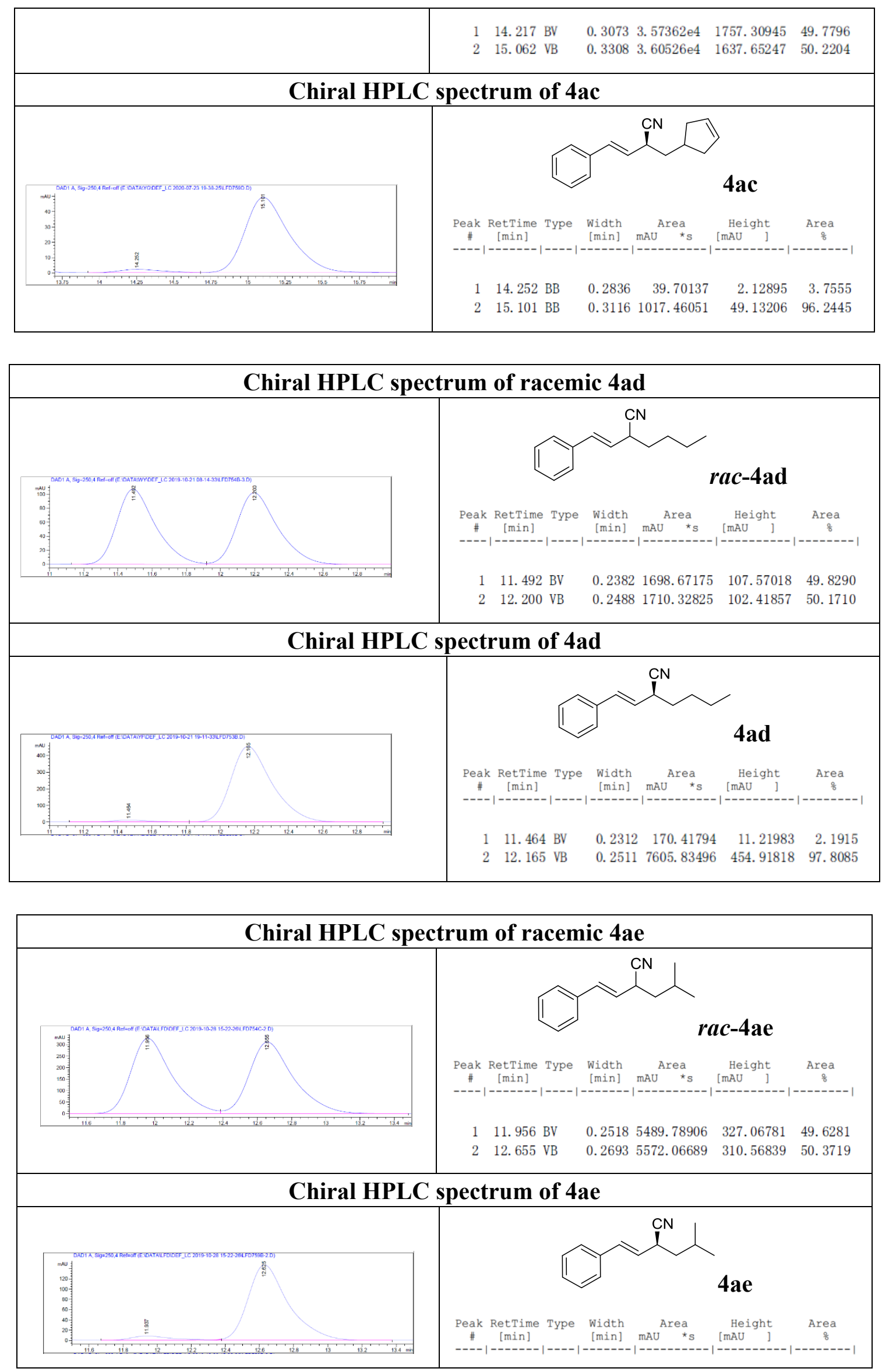

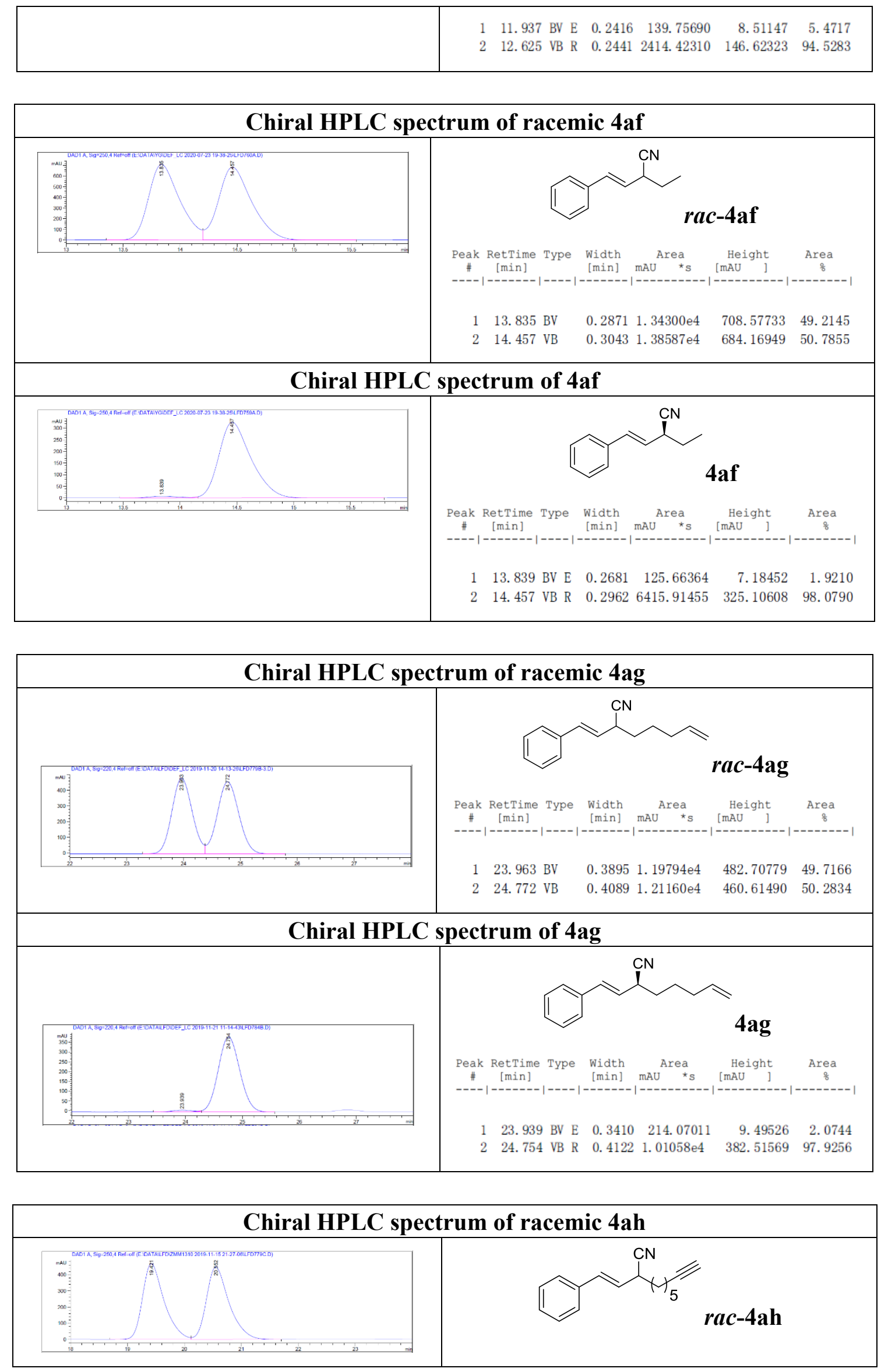

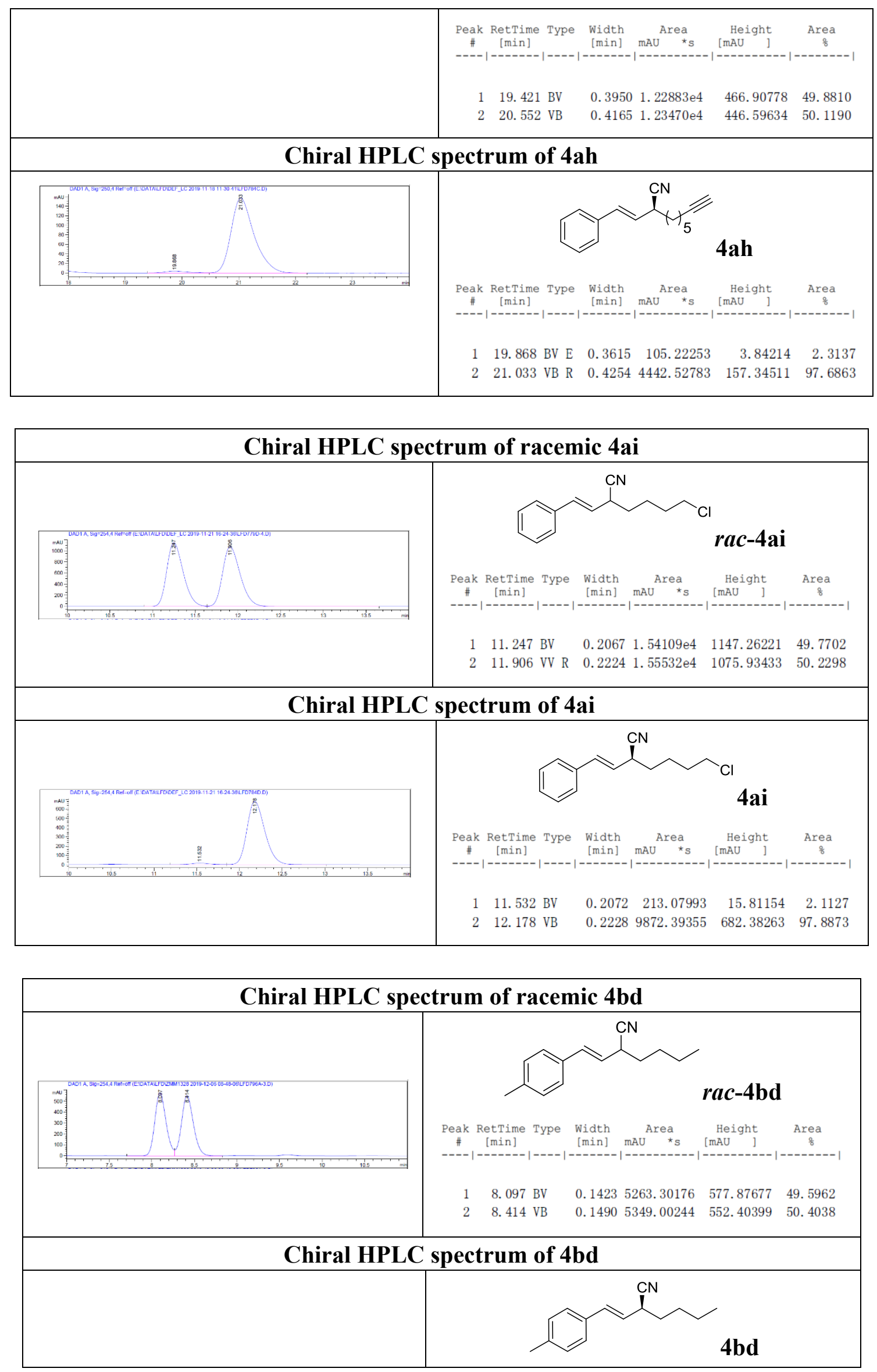

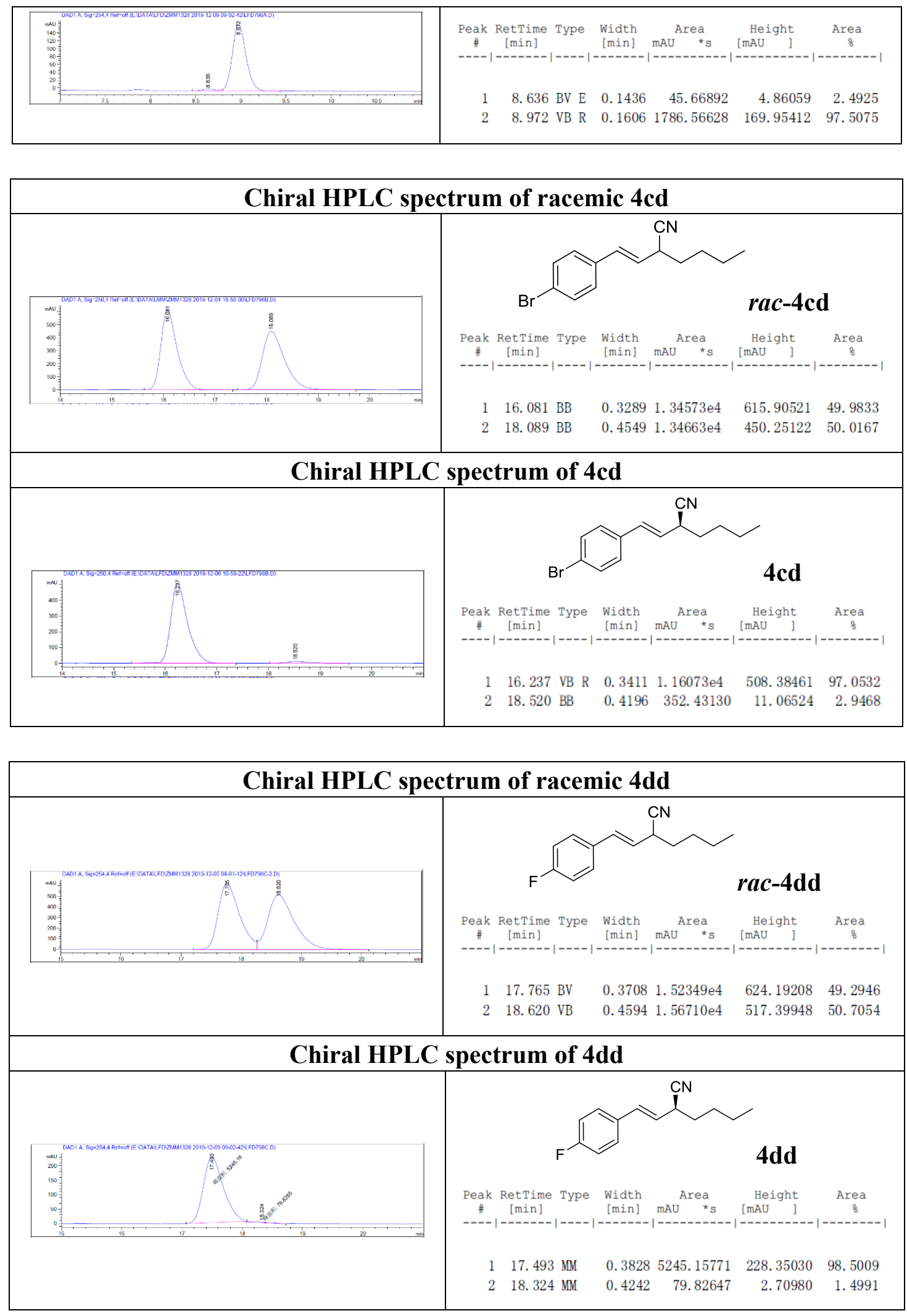

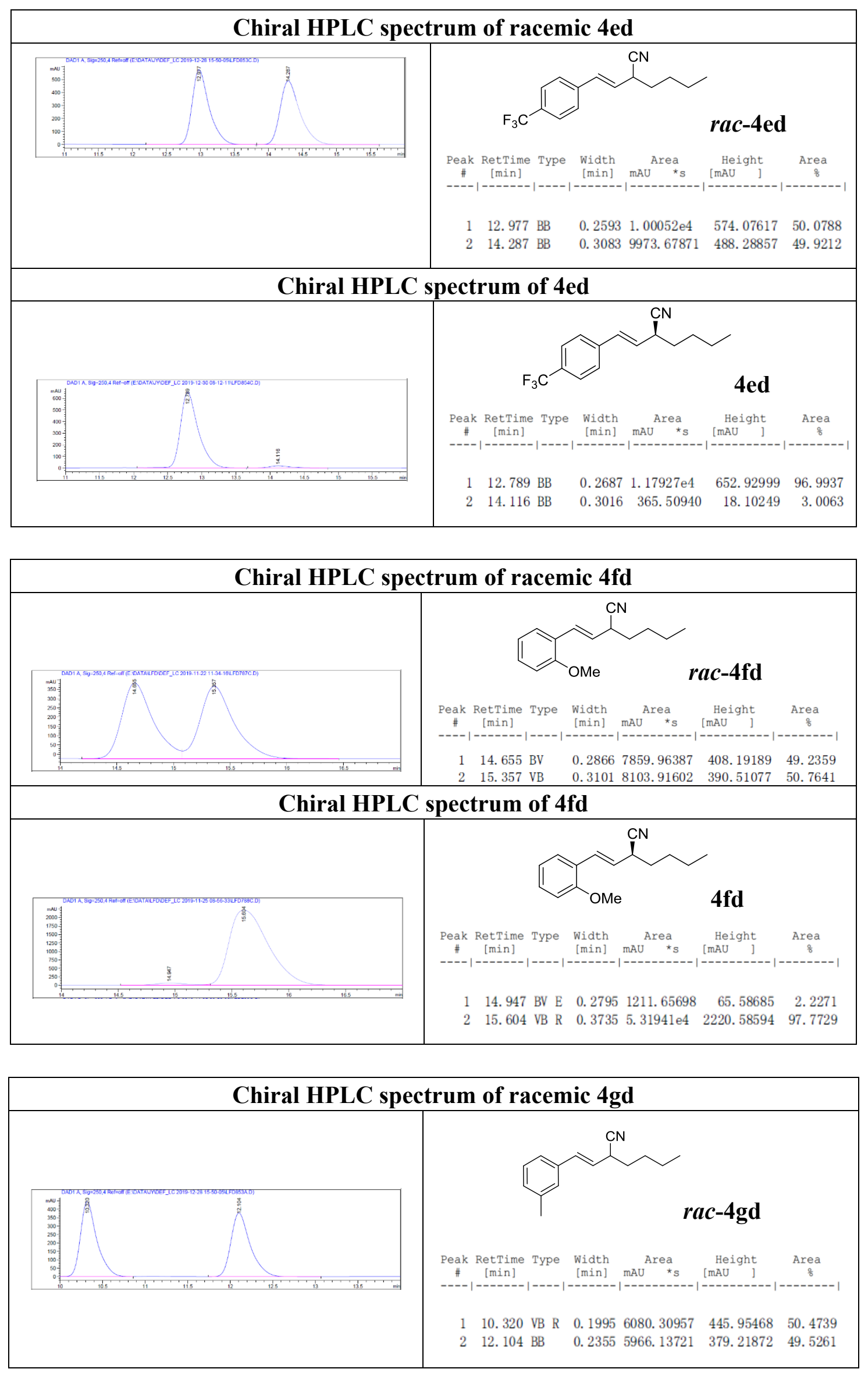

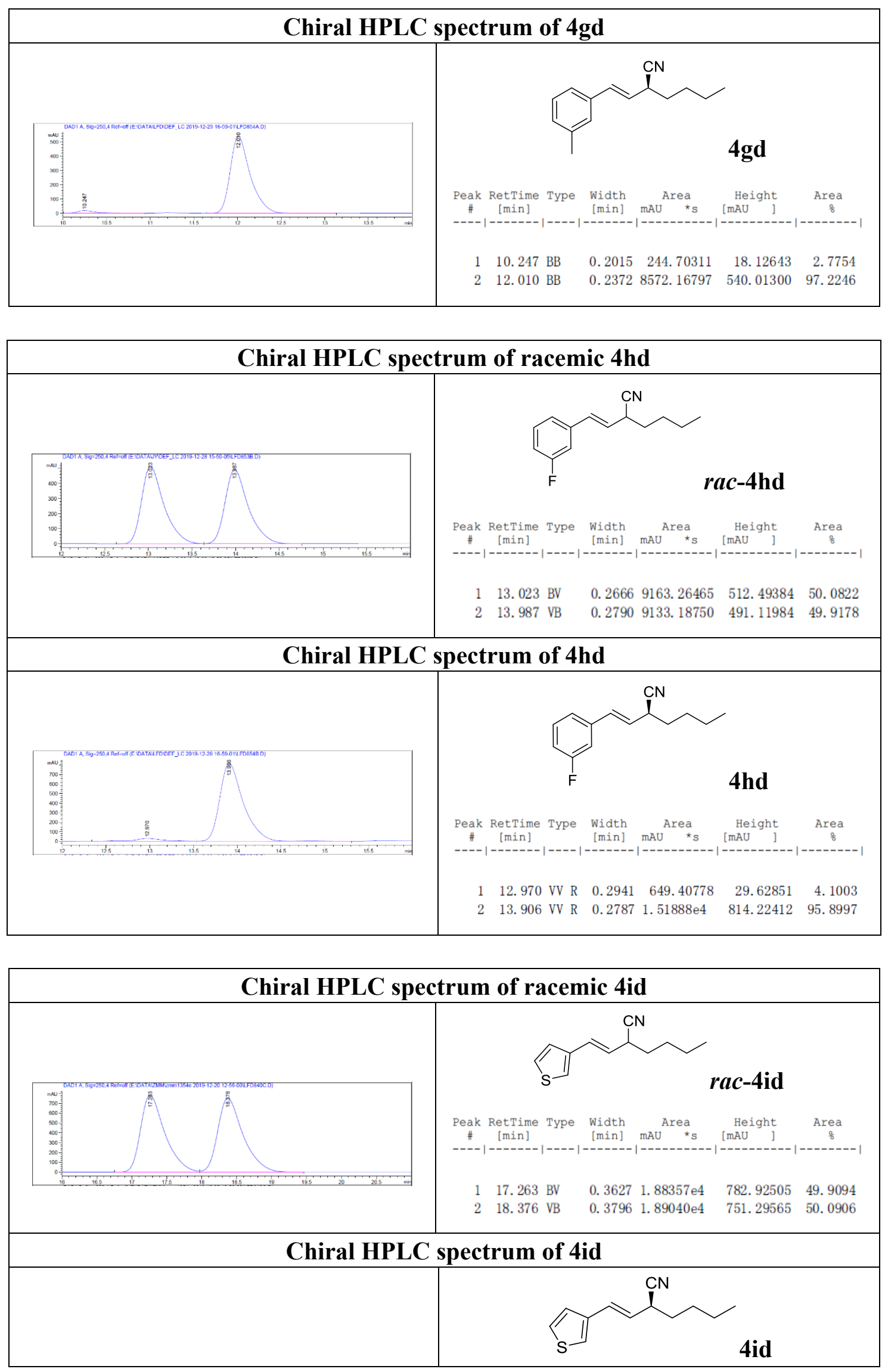

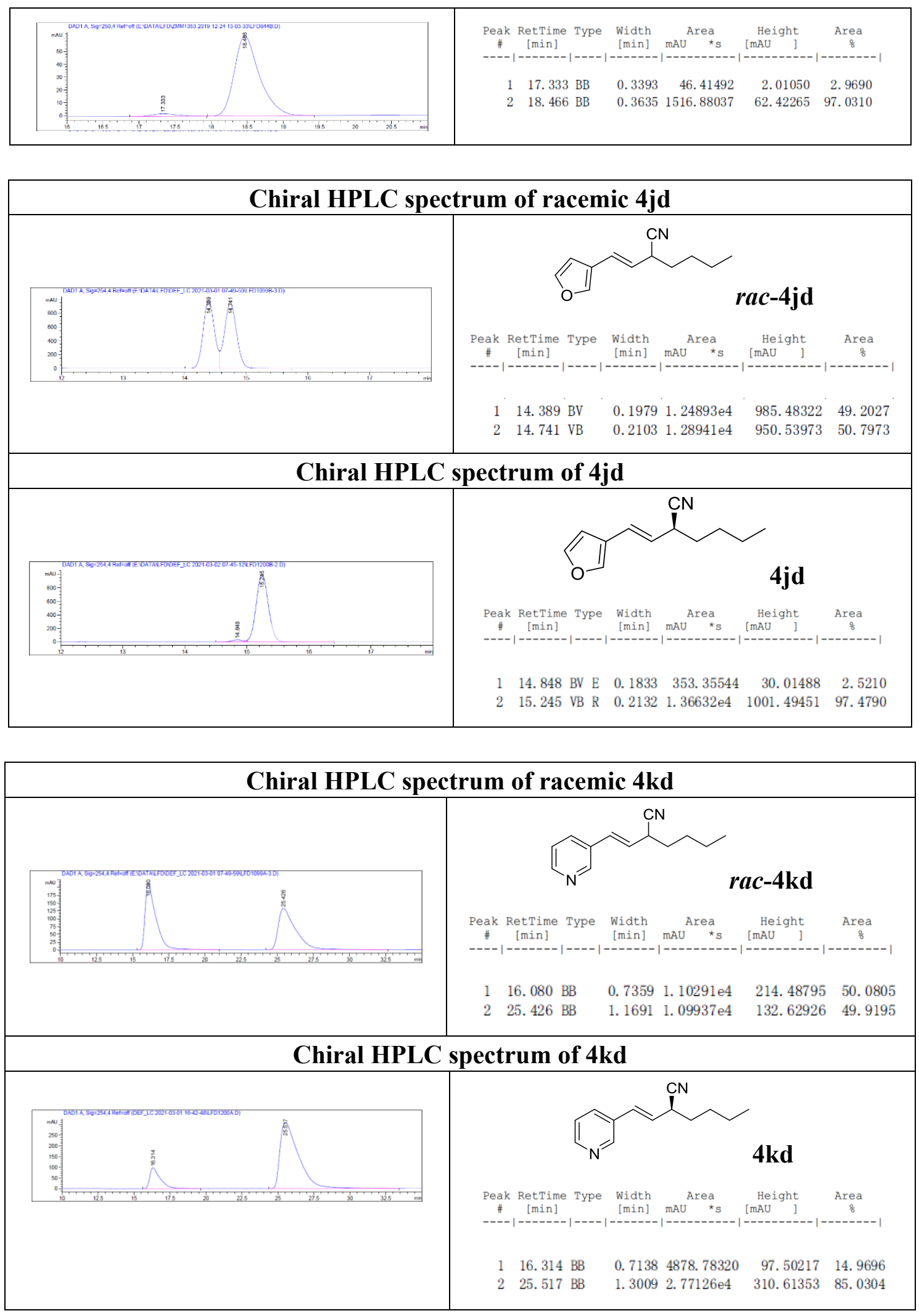

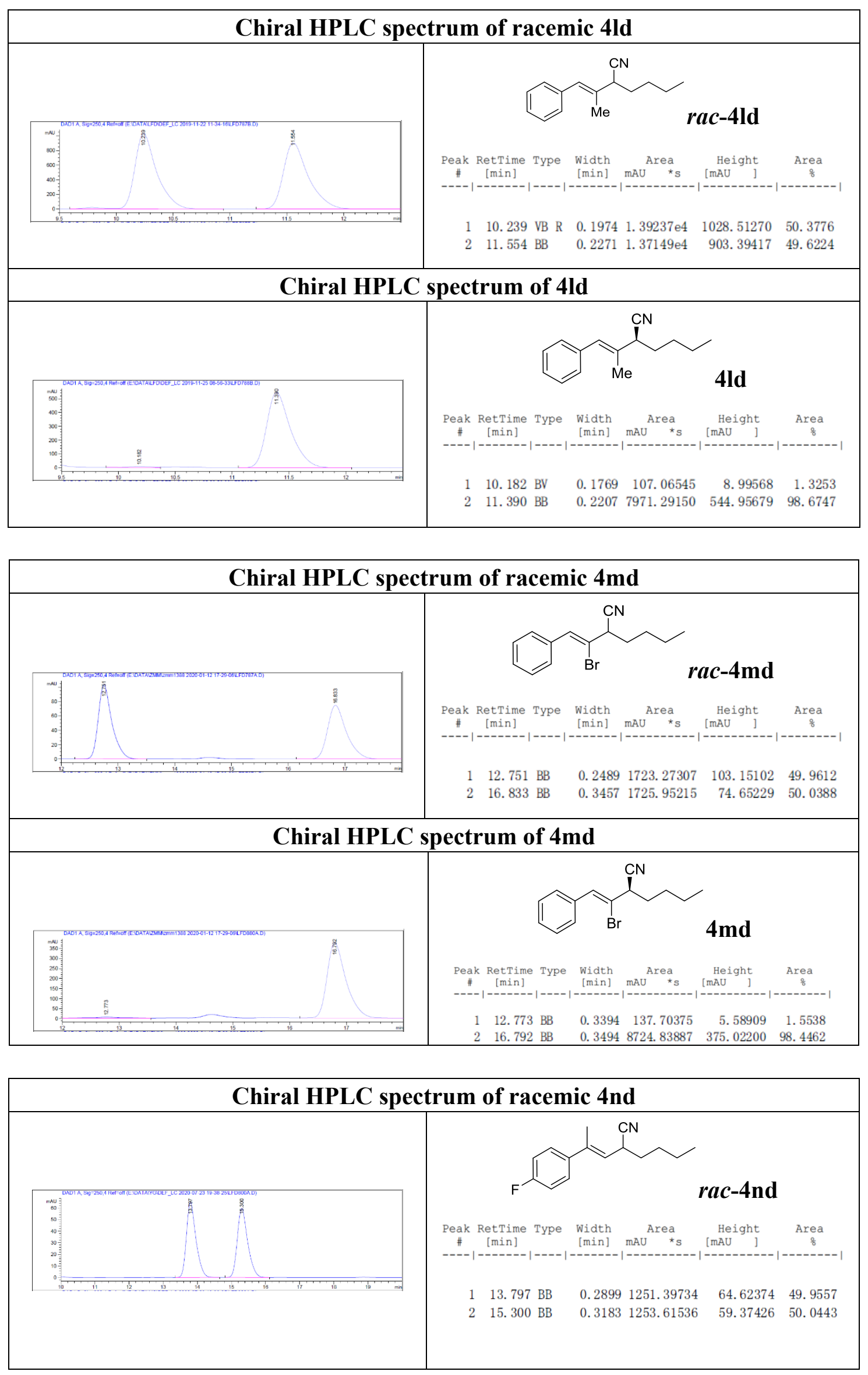

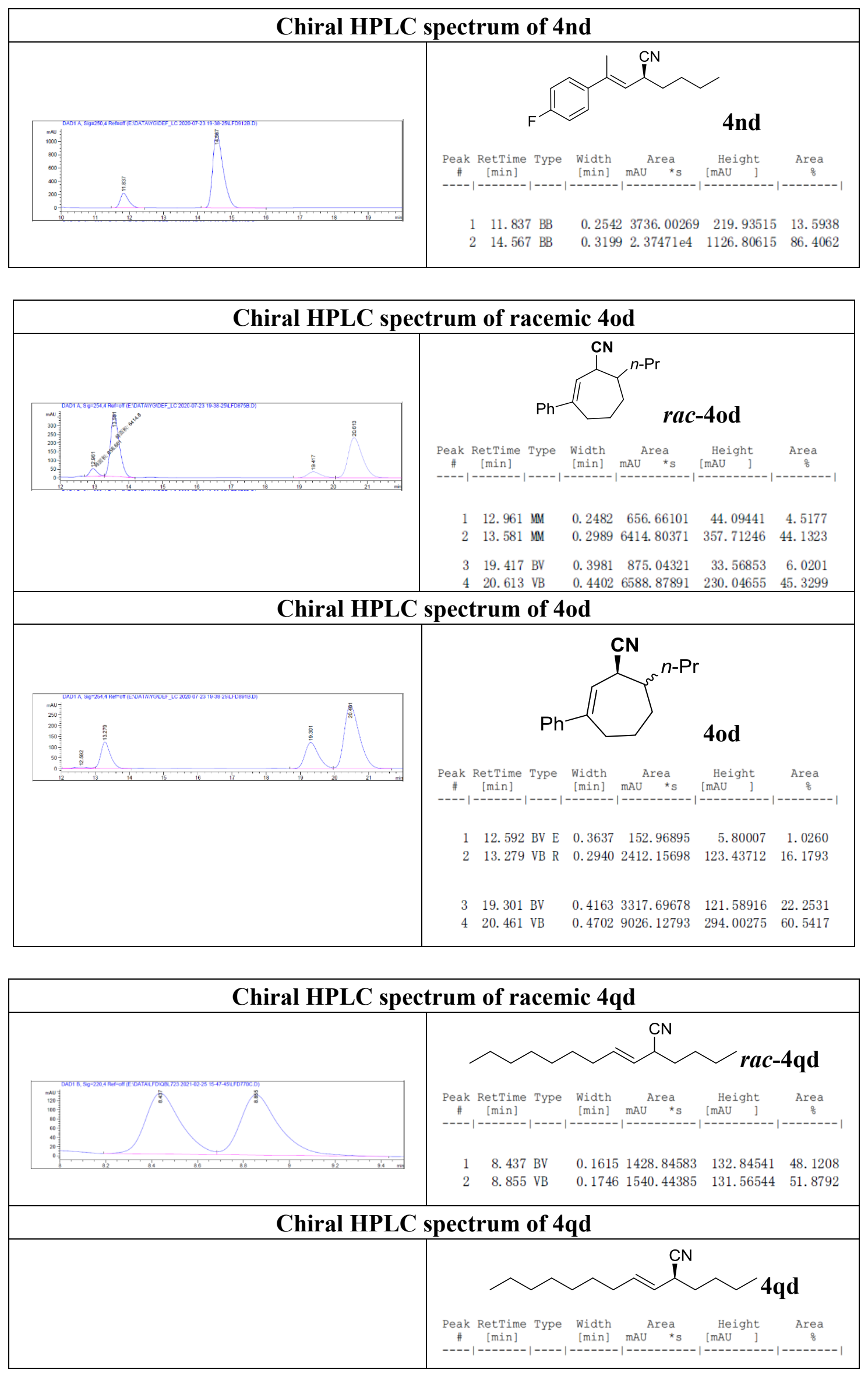


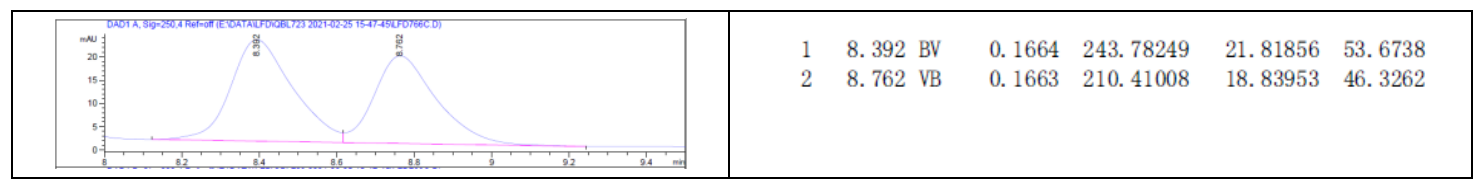
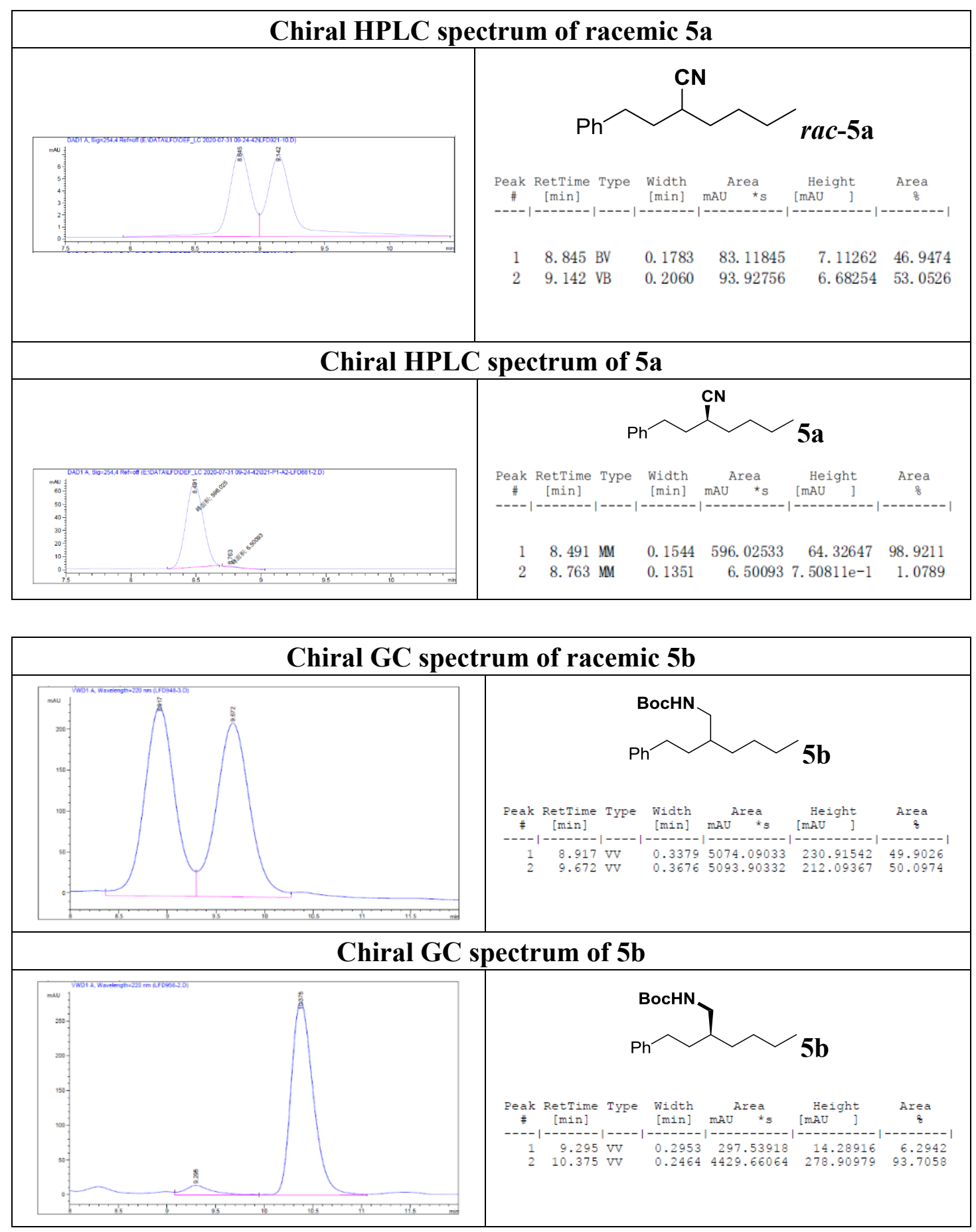

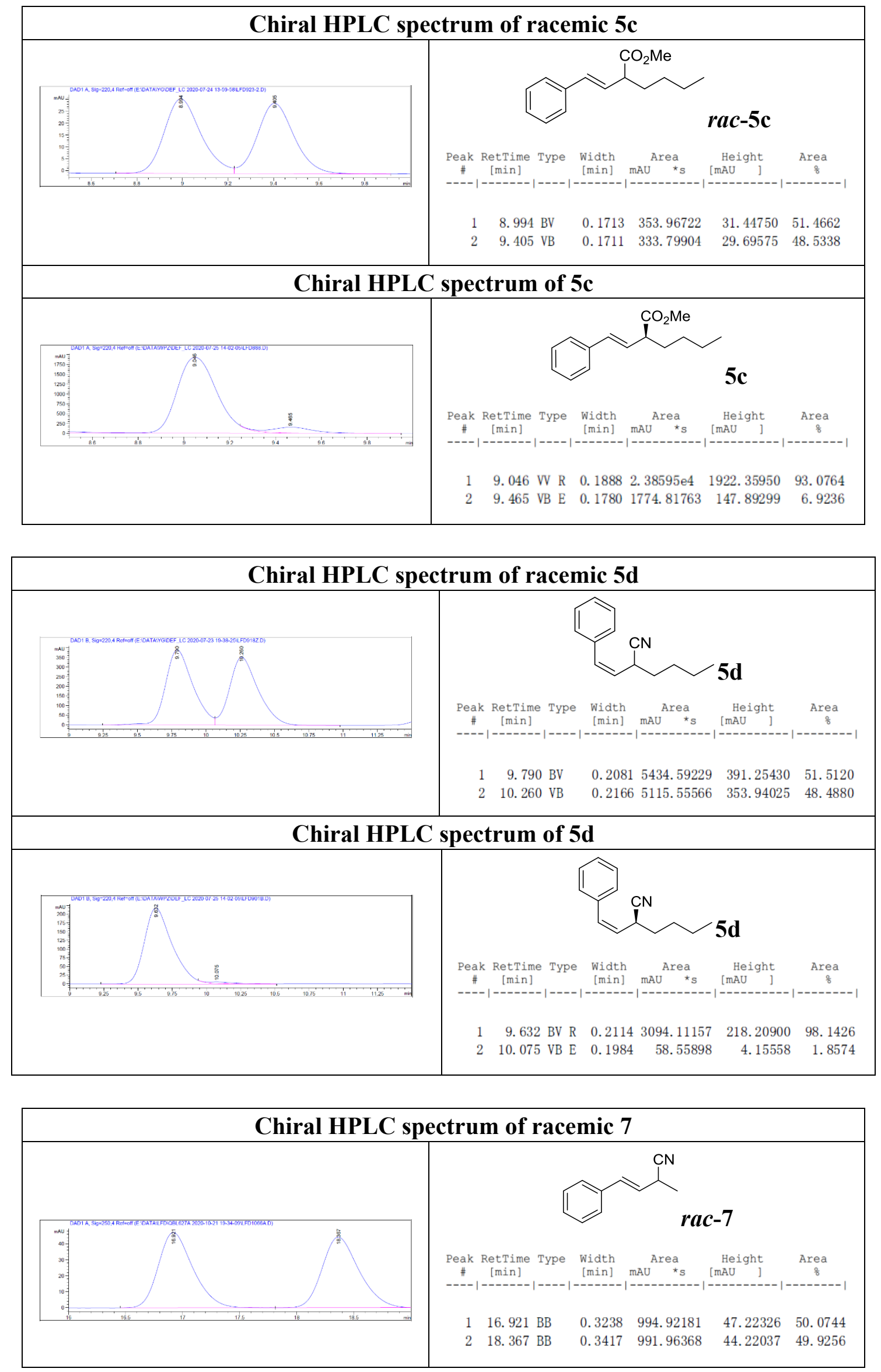

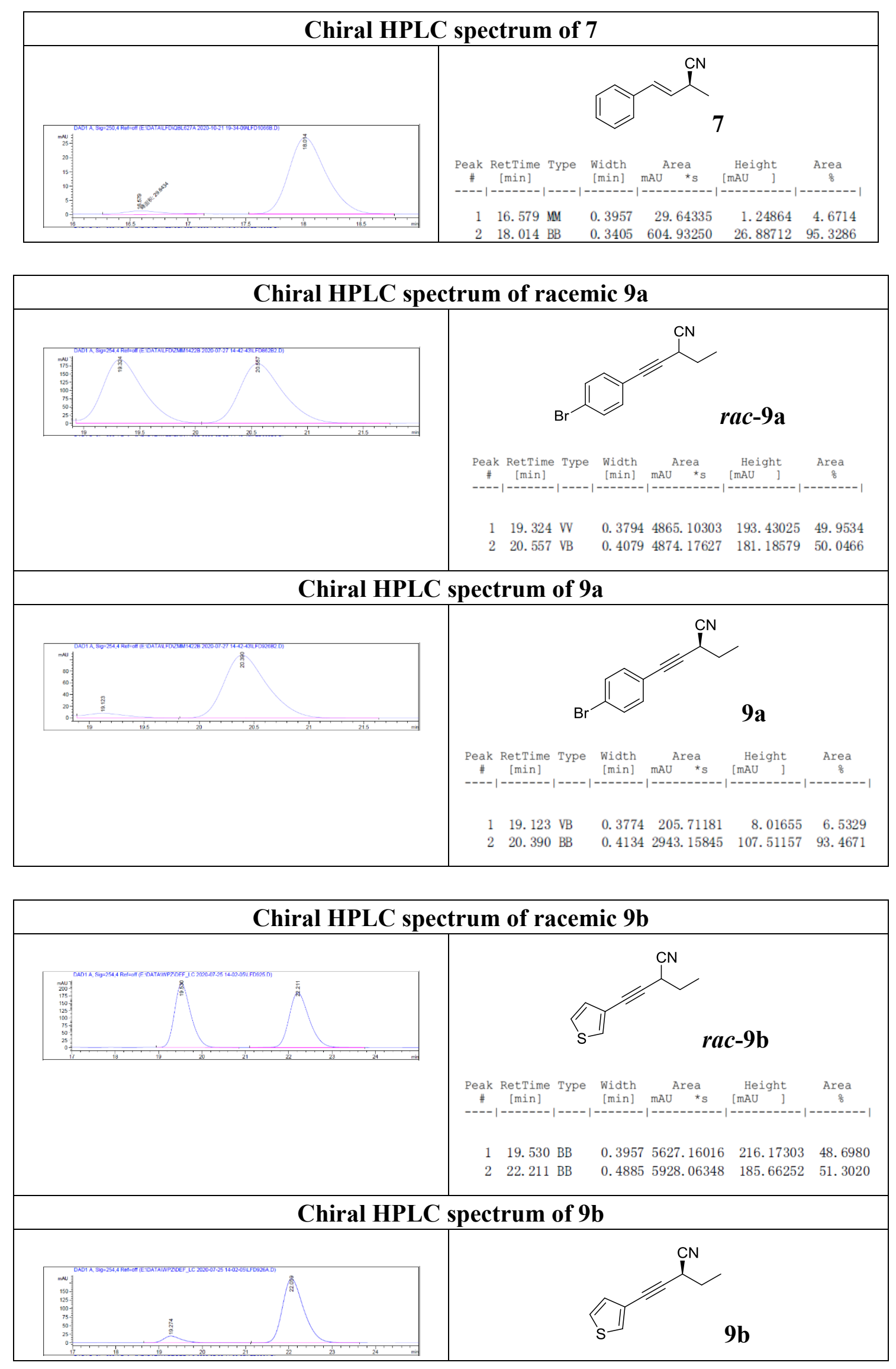

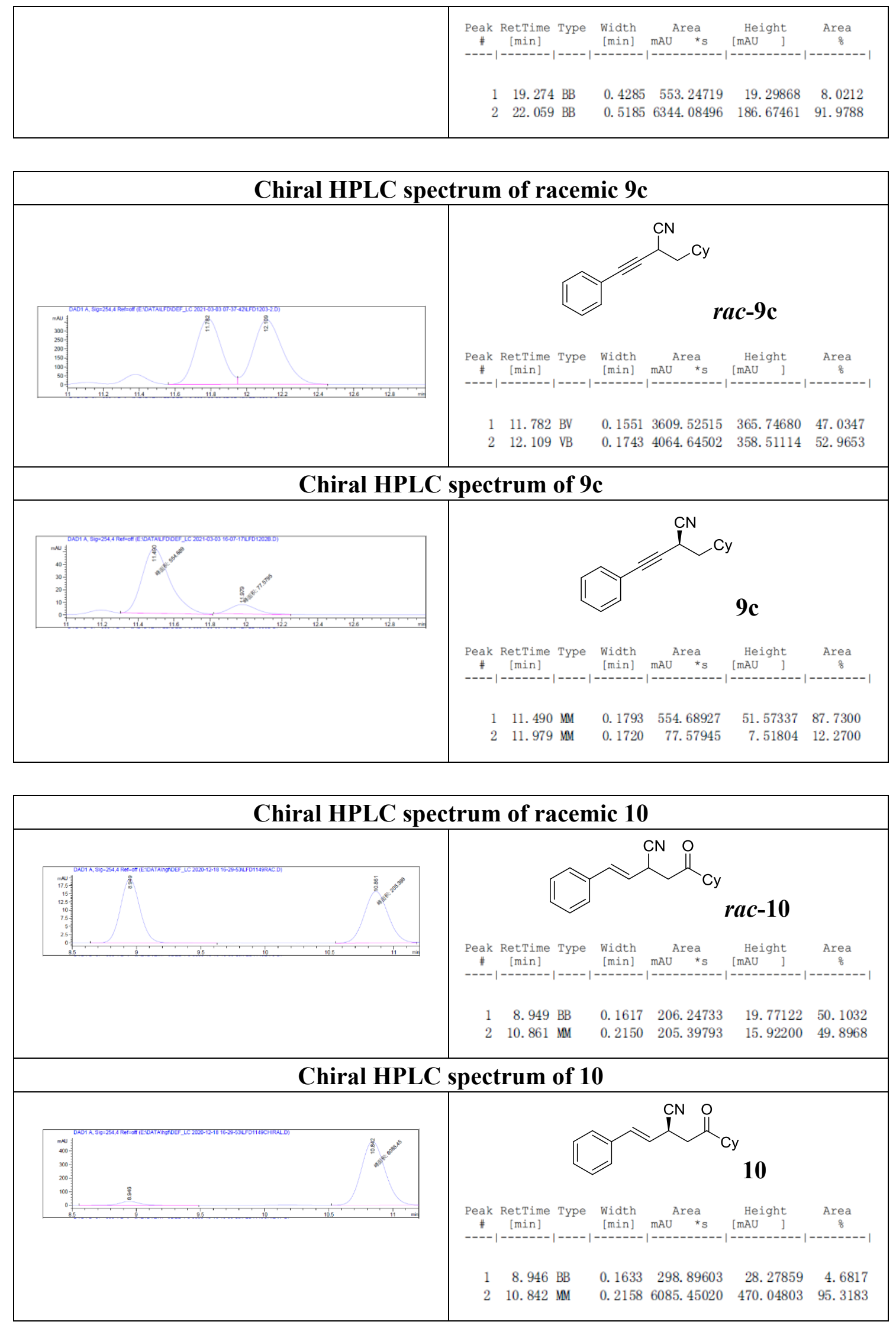


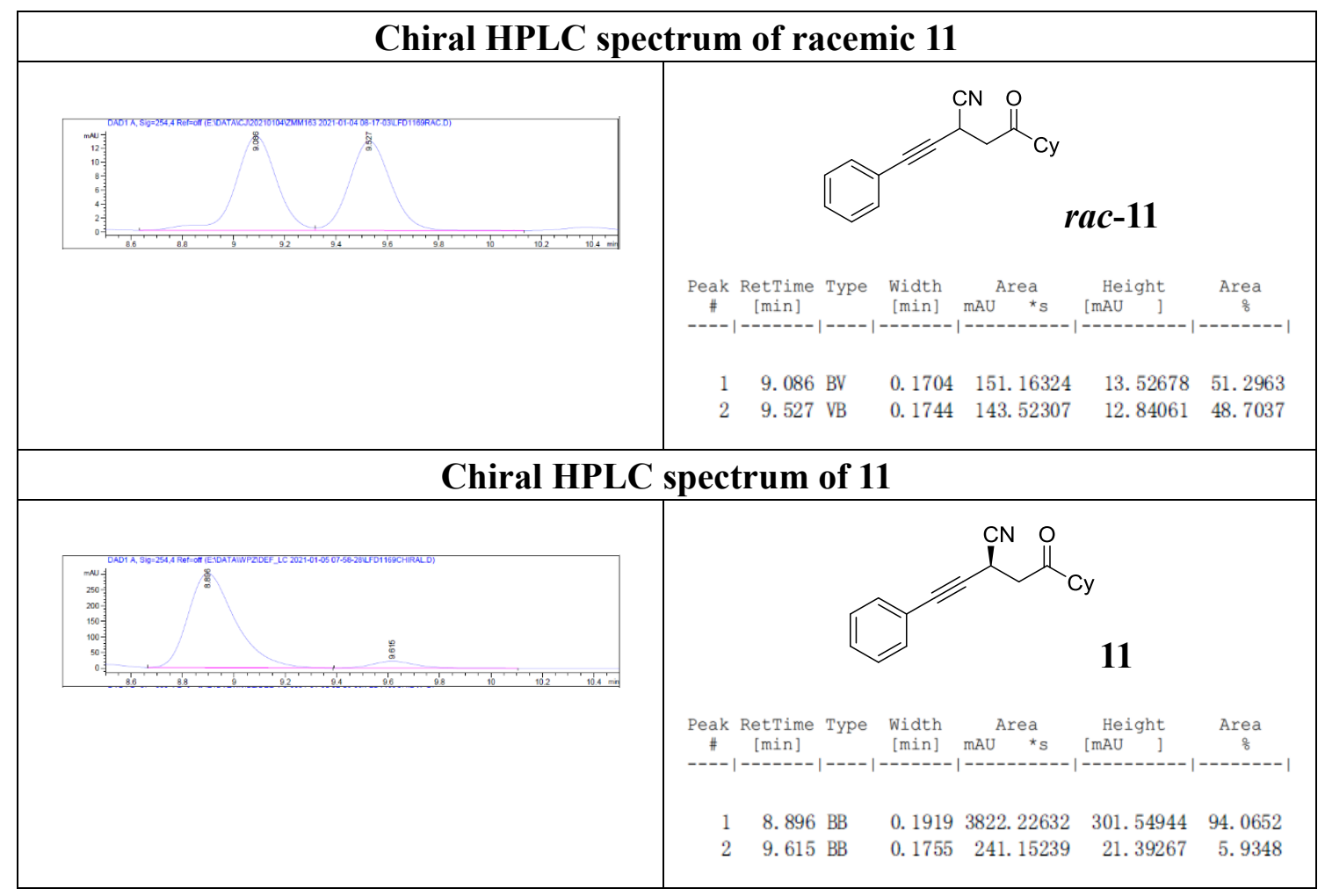

